\title{
Organic acid production in Aspergillus niger and other filamentous fungi
}

Dorett I. Odoni 


\section{Propositions}

1. Medium acidification is primarily a cause, rather than an effect, of Aspergillus niger citrate secretion.

(this thesis)

2. A holistic systems biology approach should consider the organism in the context of its environment.

(this thesis)

3. Metabolism has more than two spacial dimensions and should be studied as a system rather than a map.

4. Cancer cells behave as a microorganism in a foreign environment.

5. Referrals to authors rather than researchers highlights the skewed perception of scientific worth.

6. Reviews can both progress and regress a field.

7. Recent history suggests that "common sense" is an oxymoron.

8. True understanding, and thus friendship, arises from a common mindset rather than a common nationality.

Propositions belonging to the thesis, entitled

Organic acid production in Aspergillus niger and other filamentous fungi

Dorett I. Odoni

Wageningen, $17^{\text {th }}$ October 2017 


\section{Organic acid production in \\ Aspergillus niger and other filamentous fungi}

DORETT I. ODONI 


\section{Thesis committee}

\section{Promotor}

Prof. Dr Vítor A. P. Martins dos Santos

Professor of Systems and Synthetic Biology

Wageningen University \& Research

\section{Co-promotors}

Dr Peter J. Schaap

Associate professor, Systems and Synthetic Biology

Wageningen University \& Research

Dr María Suárez Diez

Assistant professor, Systems and Synthetic Biology

Wageningen University \& Research

\section{Other members}

Dr Sybe Hartmans, DSM Delft

Dr Arthur F. J. Ram, Leiden University

Prof. Dr Bart P. H. J. Thomma, Wageningen University \& Research

Dr Ruud A. Weusthuis, Wageningen University \& Research

This research was conducted under the auspices of the Graduate School VLAG (Advanced studies in Food Technology, Agrobiotechnology, Nutrition and Health Sciences) 


\title{
Organic acid production in Aspergillus niger and other filamentous fungi
}

\author{
DORETT I. ODONI
}

\section{Thesis}

submitted in fulfilment of the requirements for the degree of doctor at Wageningen Univesity \& Research by the authority of the Rector Magnificus

Prof. Dr A. P. J. Mol, in the presence of the

Thesis Committee appointed by the Academic Board to be defended in public on Tuesday 17. October 2017 at 4 p.m. in the Aula. 
Dorett I. Odoni

Organic acid production in Aspergillus niger and other filamentous fungi, 216 pages.

$\mathrm{PhD}$ thesis, Wageningen University \& Research, Wageningen, the Netherlands (2017).

With references, with summary in English.

ISBN: 978-94-6343-693-9

DOI: https://doi.org/10.18174/423035 
The most exciting phrase to hear in science, the one that heralds new discoveries, is not "Eureka!", but "That's funny..." -Isaac Asimov. 
1 General introduction and thesis outline 1

2 Comparative proteomics of Rhizopus delemar ATCC 20344 unravels the role of amino acid catabolism in fumarate accumulation

3 Aspergillus niger secretes citrate to increase iron bioavailability 33

4 Aspergillus niger citrate exporter revealed by comparison of two alternative citrate producing conditions

5 Aspergillus niger membrane-associated proteome analysis for the identification of glucose transporters

6 Identification and functional characterization of novel xylose transporters from the cell factories Aspergillus niger and Trichoderma reesei

7 Identification of a novel L-rhamnose uptake transporter in the filamentous fungus Aspergillus niger

8 General discussion

Bibliography

Summary 195

Overview of completed training activities 199

Acknowledgements 


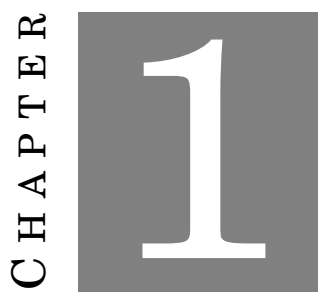

GENERAL INTRODUCTION AND THESIS OUTLINE 


\subsection{Biotechnology}

"Biotechnology" is:

"The use of living organisms or other biological systems in the manufacture of drugs or other products or for environmental management, as in waste recycling: includes the use of bioreactors in manufacturing, microorganisms to degrade oil slicks or organic waste, genetically engineered bacteria to produce human hormones, and monoclonal antibodies to identify antigens." (dictionary.com)

"The use of living organisms or biological processes for the purpose of developing useful agricultural, industrial, or medical products, especially by means of techniques, such as genetic engineering, that involve the modification of genes." (thefreedictionary.com)

"The exploitation of biological processes for industrial or other purposes, especially the genetic manipulation of microorganisms for the production of antibiotics, hormones, etc." (oxforddictionaries.com)

"The use of living things, especially cells and bacteria, in industrial processes." (dictionary.cambridge.org)

In essence, the idea behind biotechnology is that biological processes can be applied to produce a variety of compounds of interest (Figure 1.1). The exclusive use of microorganisms (or plants) to perform the conversion from substrate to product is not mandatory, but it allows a "black-box-approach" for the production of difficult-to-obtain molecules, as opposed to the application of isolated enzymes and processes derived from biology (see section 1.4).

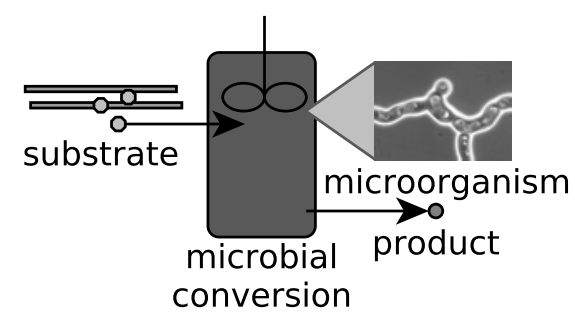

FigURE 1.1. The concept of biotechnology is to use biological processes, often microorganisms ${ }^{1}$, to facilitate conversion of available substrates to products of interest.

The concept of microbial conversion itself is older than the concept of biotechnology. However, after 1980, nascent DNA technologies (described in section 1.5.2) aided the establishment of the microbial cell-factory concept. With the advent of

\footnotetext{
${ }^{1}$ The microscopy image of A. niger was taken by Jasper Sloothaak.
} 
the accompanying metabolic engineering technologies, so called "first-generation" cell-factories, in which plain strains of the given micro-organisms were used for their natural products, are now being replaced by engineered variants (Lee et al., 2012).

\subsection{Organic acids}

One of the applications of biotechnological cell-factories is the production of organic acids. Organic acids are low-molecular-weight organic compounds with one or more ionisable functional groups, such as - $\mathrm{COOH}$, $-\mathrm{OH}$ or $-\mathrm{SH}$. The chemical property of these groups to donate (or accept) protons is what makes organic acids in effect acidic, although they are considered to be weak acids, and as such do not dissolve completely in water. In this thesis, I will focus on carboxylic acids, mainly fumaric and citric acid.

\subsubsection{Fumaric acid}

Fumaric acid, or fumarate ${ }^{2}$, is a symmetric dicarboxylic acid. It was discovered independently by Braconnet and Vauquelin in 1817, who found it after dry distillation of malic acid (Goldberg et al., 2006). Fumarate is found in plants of the genus Fumaria, from where it derives its name. It is used as an intermediate in the production of other organic acids such as L-malic or L-aspartic acid, and is an important intermediate for polymer production (Goldberg et al., 2006).

The production of fumarate currently relies on chemical synthesis from maleic anhydride, which in turn is produced from butane, a component of crude oil (Engel et al., 2008). Although the chemical process is cheaper and more efficient than biolocical conversion, the associated environmental impact has resulted in a new surge of interest for biological fumarate production (Meussen et al., 2012).

\subsubsection{Citric acid}

Citric acid, or citrate, is a symmetric tricarboxylic acid, first isolated and crystallised from lemon juice by Carl Wilhelm Scheele in 1784 (Goldberg et al., 2006). It derives its name from the Latin word "citrus", which, rather than referring to the lemon fruit, is the Latin name of the citron tree (a slow-growing shrub that produces lemon-like fruit). Citrate is used, amongst others, as food acidu-

\footnotetext{
${ }^{2}$ Throughout this thesis, I did not make the distinction between the the protonated (acid) or the deprotonated (-ate) form of the compounds discussed
} 
lant, in cleaning agents, hygiene and cosmetic products, pharmaceuticals, and as pesticide.

Commercial production of citrate started in England around 1826, where it was extracted from Italian lemons (Goldberg et al., 2006). As the commercial importance of citrate grew, so did the demand for lemons. The resulting success of lemon exports from what is still known as the "Conca d'Oro" (Golden Bay) around Palermo, Sicily, quickly turned sour with the need to maintain strategic lemon monopoly. This gave rise to unprecedented use of violence in the area, concealed by the high walls that shielded both the treasured lemon gardens and the hitmen that were ensuring business was "run as agreed on" (From "The Land Where Lemons Grow: The Story of Italy and Its Citrus Fruit").

In 1893, Carl Whemer reported the first instance of citrate production using fungi he named Cytromyces (later Penicillium). At the time, Wehmer was also studying oxalate production in a mould called Aspergillus niger, but it was not until World War I interrupted Italian lemon exports that an American chemist, James Currie, was driven to experiment with different $A$. niger strains in order to find alternatives for the broken supply chain of citrate production. The establishment of the conditions that increased the yield of citrate over oxalate in A. niger (Currie, 1917) laid the groundwork for biotechnological citrate production, and the first industrial citrate production process using $A$. niger began in Belgium in 1919 (Goldberg et al., 2006).

\subsection{Aspergillus niger and other (filamentous) fungi}

Currently, more than $99 \%$ of global citrate production is obtained from microbial sources, and although some yeast strains are used for the biotechnological production of citrate (Magnuson and Lasure, 2004), $80 \%$ is obtained by submerged fermentation using the filamentous fungus $A$. niger (Dhillon et al., 2011). Filamentous fungi, in contrast to yeast, grow in multicellular filaments called hyphae, and play an important role in the decomposition of plant material due to their ability to secrete hydrolytic enzymes (de Vries and Visser, 2001).

The introduction of the microbial process to obtain citrate broke the dependency on Italian lemons, but citrate production is still subject to fierce competition, and the actual industrial strains and production conditions for citrate are not publicly available (Karaffa and Kubicek, 2003). Nonetheless, A. niger citrate production has been studied in academia, and the culture conditions and enzymes that are relevant for $A$. niger citrate production have been identified and, partially, explained. For an extensive overview, see Show et al. (2015) and Yang 
et al. (2016).

Besides its importance as biotechnological citrate producer, $A$. niger is also an important cell-factory for the production of other organic acids and hydrolytic enzymes (Andersen et al., 2011), and for the removal of toxic heavy metals as a form of waste water treatment (Tsekova et al., 2010). In contrast to most prokaryotes, $A$. niger is able to not only secrete but also tolerate high concentrations of acids in the medium and thus low $\mathrm{pH}$, thereby facilitating product recovery (Yin et al., 2015). Like other filamentous fungi that decompose plant (waste) material, A. niger is an efficient degrader of lignocellulose (de Vries and Visser, 2001) and can therefore utilise second generation feed stocks, making this fungus an ideal cell-factory for the sustainable production of various compounds of interest (Lubertozzi and Keasling, 2009).

As an example, we were interested in the possibility to establish $A$. niger as microbial cell-factory for the production of fumarate, which $A$. niger secretes only in trace amounts. The filamentous fungus Rhizopus delemar, on the other hand, secretes relatively high amounts of fumarate, and before the establishment of fumarate production via chemical conversion, Rhizopus spp were used as biological fumarate producers (Goldberg et al., 2006). In contrast to A. niger, however, $R$. delemar is regarded as opportunistic human pathogen with an aggressive course of infection, and its genetic makeup (Ma et al., 2009) makes it unsuitable for genetic modification.

A. niger is genetically more amenable than $R$. delemar, but metabolic engineering approaches aimed at changing the $A$. niger organic acid production profile are only moderately successful. Thus far, none of the reported genetic modifications managed to re-route metabolic flux completely away from citrate to other organic acids, nor did optimisation of the culture conditions (Yang et al., 2016).

\subsection{The main elements of microbial cell-factories}

In order to successfully engineer A. niger to become a better cell-factory for a wider range of products, we have to understand the separate elements that are necessary to produce any given compound of interest. Roughly, there are 3 main processes that lead from substrate to producct in any given microbial cell-factory: Import of the substrate into the cell, conversion of substrate to the product of interest, and export of the product of interest out of the cell (Figure 1.2).

The conversion of substrate to product using the microorganism as a whole, i.e. effectively as cell-factory, distinguishes the approach from the use of biological 


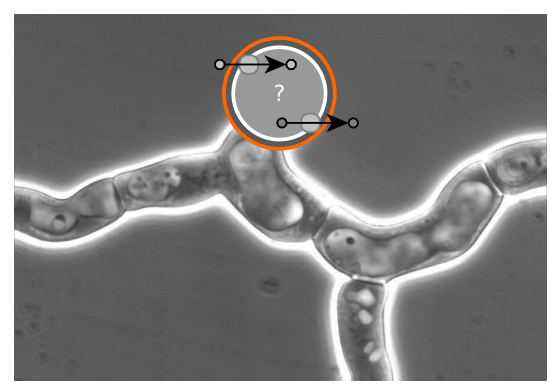

FiguRE 1.2. Essential elements of microbial cell-factories - substrate import, conversion, and product export.

processes that are merely derived from cells, such as the enzymatic conversion of glucose to gluconate by using the $A$. niger glucose oxidase as catalyst (Neves and Vitolo, 2007). The benefit of using the microorganisms itself is that biological conversion can be quite complex (rather than just one or a few enzymes), and we do not necessarily have to understand every single step of import, conversion, and export, i.e. we can treat the organism as black-box while we unravel the processes underlying each element one-by-one.

Besides the 3 elements outlined in Figure 1.2, cellular compartmentalisation plays an important role in any metabolic engineering approach. As a consequence of eukaryotic compartmentalisation, compounds have to be transported to and from the location of the actual conversion which, in the case of organic acids, often happens in the mitochondria (see section 1.4.2). The importance of intracellular transport can thus not be disregarded (van der Straat and de Graaff, 2014).

\subsubsection{Substrate import into the cell}

For sustainable biotechnological production of organic acids and other compounds of interest, we want to use plant waste material, thereby avoiding competition with food production. As natural decomposers of plant biomass, Aspergilli can degrade and absorb a wide range of carbon sources found in for mammals hard to digest plant (waste) material. Ideally, the cell-factory should use all the carbon available in plant biomass, and identification and characterisation of $A$. niger substrate import proteins can provide valuable tools for improvement of substrate utilisation. 


\subsubsection{Microbial conversion of substrate to product}

Once inside the cell, the substrate has to be converted to the product of interest. The conversion takes place via enzymes, which can be grouped into metabolic pathways, which in turn can involve multiple steps and transport across different cellular compartments, until the substrate is converted to the product we are interested in.

Most organic acids of industrial interest are intermediates in naturally occurring metabolic pathways, of which the (reductive) tricarboxylic acid ((r)TCA) cycle is the best known (Figure 1.3).

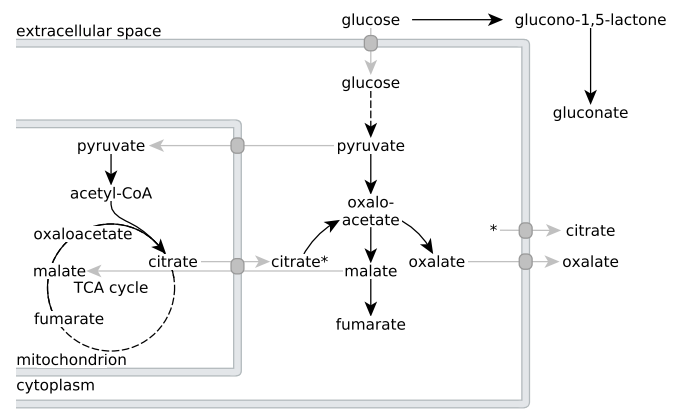

FIGURE 1.3. Organic acid production in filamentous fungi.

Both citrate and fumarate are intermediates in the TCA cycle, which is located in the mitochondria. Fungal fumarate accumulation, however, is attributed to enzymes of the rTCA cycle in the cytosol (Goldberg et al., 2006; Wright et al., 1996). Reductive and oxidative TCA cycles are connected by the pyruvate branch-point, which is an important cross-road for many metabolic engineering approaches as, depending on the culture conditions, pyruvate will be converted into a number of different compounds (Meussen et al., 2012).

\subsubsection{Product export from the cell to the extracellular medium}

Another component of control can be the export of the product of interest out of the cell. Although technically not as mandatory a step as substrate import and conversion, citrate export is suggested to be the bottleneck of $A$. niger citrate production (Karaffa and Kubicek, 2003). Similarly, production of the C4-dicarboxylic acids fumarate, malate and succinate is suggested to be bottlenecked at the transport level across various cell-organelles and into the extracellular medium (Meussen et al., 2012). 


\subsection{Systems biology}

\subsubsection{Reductionism and holism}

Identification and analysis of the separate elements of the system is considered a reductionistic approach of biology, specifically methodological reductionism (as opposed to epistemological reductionism, in which one scientific discipline, e.g. biology, can be reduced the principles of another scientific domain, e.g. physics and/or chemistry, or ontological reductionism, which boils down to physical matter being the only reality in nature (Fang and Casadevall, 2011)). Although useful, reductionism is biased towards giving too much explanatory weight to single factors, and thereby disregards the immense complexity of any biological system (Van Regenmortel, 2004).

The shortcomings of the reductionist approach is exemplified by the unpredictability of success or failure in metabolically engineering $A$. niger for increased citrate production. While overexpressing enzymes involved in glycolysis and the TCA cycle did not result in higher citrate production (Ruijter et al., 2000, 1997b), metabolic engineering of the cytosolic rTCA cycle resulted in increased citrate yield and productivity (De Jongh and Nielsen, 2008). Most remarkably, metabolic engineering approaches to prevent formation of gluconate and oxalate, which are considered unwanted by-products during citrate production, led to major readjustments of $A$. niger citrate production, as the resulting mutants accumulated citrate in the extracellular medium even when the $\mathrm{pH}$ was "high" (5) and in the presence of $\mathrm{Mn}^{2+}$ ions; conditions that usually prevent the accumulation of citrate in favour of the other two organic acids gluconate and oxalate (Ruijter et al., 1999). This remarkable change suggests a more complex interplay of organic acid production than just the mere interruption of pathways.

Following the saying that "The whole is greater than the sum of its parts" (Aristotle), the concept of emergent properties of any complex biological system has led to the development of the more holistic approach of systems biology (Van Regenmortel, 2004). The term "holism" itself was coined by Smuts as "a tendency in nature to form wholes that are greater than the sum of the parts through creative evolution" (Fang and Casadevall (2011), from "Holism and evolution" by J.C. Smuts). A fundamental principle underlying systems biology is that cellular and organismal constituents are interconnected (Fang and Casadevall, 2011), and we need to study the system as a whole, rather than just the isolated parts, in order to understand it. 


\subsection{2 -omics}

According to Joyce et al., the transition from reductionism to holism, or from biology as a discipline that was relatively data-poor to a discipline that was suddenly very data-rich, was marked by the completion and publication of the Haemophilus influenzae genome sequence by Fleischmann et al. in 1995 (Joyce and Palsson, 2006). Ever since, genome sequencing has become faster and cheaper; while sequencing of the first human genome, launched in 1990, took 13 years to complete, 1'092 human genomes were sequenced from 2008 - 2012 in the context of the 1'000 human genomes project. Similarly, the price for sequencing has dropped considerably, from a cost per genome of 100'000'000 \$ in 2001 to 1'000 \$ in 2015 (or 10 '000 $\$$ per raw mb of DNA sequence to $<0.1 \$$, tracked by the National Human Genome Research Institute: https:/www.genome.gov/sequencingcostsdata/).

The advent of the high-throughput DNA sequencing technologies was followed by the development of the so called "-omics" technologies, in which genome-scale measurements for many of the cellular components were made possible. Omics data can describe the cell based on three broad categories: components, interactions, and functional states (Joyce and Palsson, 2006). The components of the cell are, amongst others, the genome itself, and the derived transcriptomes, proteomes and metabolomes. Interactions can be protein-DNA interactions and proteinprotein interactions, and functional states can be described by the metabolic fluxes and the phenotype. In this thesis, I worked exclusively with components data analysis.

The theoretical potential of any living organism is encoded in its DNA, the entirety of which makes up the genome of an organism. Genomics describes the study of the full genome sequence and the information stored within. In this, the annotation of the genome gives valuable information about the proteins and functional RNAs encoded, as well as regulatory elements controlling these (Joyce and Palsson, 2006). The information of whole genomes gave rise to the emergence of genome-scale metabolic models, which are mathematical representations of the knowledge on an organism's metabolism (Patil et al., 2004).

More than the metabolic potential of the organism, we will often be interested in the actual state of the organism given an external perturbation. As a response to the environment, gene expression will be modulated to allow for adaption, and capturing the changes on transcript level will give a closer look at what is important under a certain condition. An indication of active components in the cell can therefore be given by transcriptomics, bearing in mind that study of the organisms' transcriptomic landscapes disregards post-transcriptional regulation 
and thus control.

Proteomics aims to identify and quantify relative abundances of proteins, sometimes in combination with their sub-cellular location, and is thus similar to transcriptomics in that it provides an overview of the actual status quo of the cell. However, similarly to trancriptomics approaches, presence of a given protein does not necessarily equal activity and thus flux through the metabolic pathways identified, and post-translational modification and control is not captured, either.

Nonetheless, large scale differences in metabolic pathways on transcriptome and proteome level can often be connected to contrasting observed phenotypes, as will become apparent throughout this thesis. Another important point is that reductionistic and holistic approaches in biology are not mutually exclusive, and as such are not truly opposed to each other. In this thesis, both principles were applied synergistically. 


\subsection{Aim and thesis outline}

The aim of this thesis was to increase the understanding of organic acid production in Aspergillus niger and other filamentous fungi, with the ultimate purpose to improve $A$. niger as biotechnological production host. At the core of this thesis, I focussed on understanding organic acid production in the context of the filamentous fungus producing the organic acid studied, i.e on the conversion step. However, important advances in identifying different $A$. niger sugar importers, and the identification of a promising $A$. niger citrate exporter candidate, are also described (Figure 1.4).

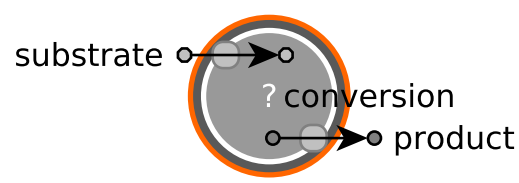

FIGURE 1.4. To effectively improve $A$. niger as microbial cell-factory, we want to identify key components of substrate import and product export, and gain a deeper understanding of the metabolic conversion from substrate to product inside the cell.

This Chapter (Chapter 1) gives a general introduction to the challenges and methods treated in this thesis.

In Chapter 2, we studied differences in the transcriptional and proteomic responses of $R$. delemar under high and low fumarate producing conditions, with the goal of increasing both the understanding of $R$. delemar fumarate production, and identifying a possible candidate fumarate export protein for heterologuous expression in A. niger. Although we failed to identify the correct transporter (discussed in Chapter 8), the results of these analyses lead to a broader understanding of the mechanism underlying fumarate accumulation in R. delemar.

In order to make $A$. niger a suitable production host for other organic acids, we also delved deeper into the understanding of why this fungus is a natural acidifier in the first place. Our observations led us to the conclusion that increased citrate secretion under iron limitation in gluconate and oxalate non-producing $A$. niger mutants is consistent with a role of citrate as iron siderophore, described in Chapter 3.

In Chapter 4, we continue our quest to understand the various aspects of A. niger citrate production, and show that it is the result of multiple different 
underlying mechanisms, thereby providing a framework for the discussion why so many conflicting reports regarding the underlying mechanism exist. In addition, we combined the data obtained from Chapters 3 and 4 to compile a list of putative citrate exporter candidates, and tested two of these in Saccharomyces cerevisiae.

For the identification of different $A$. niger substrate importers, we combined in silico and in vivo approaches, and established a reliable pipeline to identify and test candidate transport proteins. The in silico approach, in which likely glucose transporter candidates are inferred from good matches with a glucose transporter specific hidden Markov model ( $\left.\mathrm{HMM}_{\mathrm{gluT}}\right)$, and the in vivo approach, in which a sub-cellular proteomics approach is applied to isolate plasmalemmal glucose transporters, is described in Chapter 5.

The concepts developed in Chapter 5 were applied to identify further substrate importer proteins in both $A$. niger and Trichoderma reesei, another filamentous fungus. Our findings, described in Chapters 6 and 7, show that our approach is a robust pipeline to identify different substrate importer candidates.

In Chapter 8, I summarise the findings of this thesis with regards to our attempts at improving $A$. niger as biotechnological production host, and discuss the thesis in the broader context of future metabolic engineering strategies. 


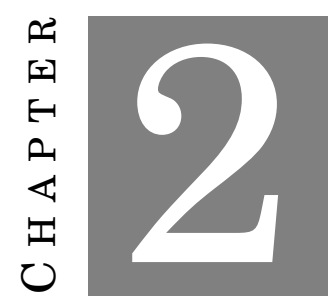

COMPARATIVE PROTEOMICS OF Rhizopus delemar ATCC 20344 UNRAVELS THE ROLE OF AMINO ACID CATABOLISM IN FUMARATE ACCUMULATION

This chapter is adapted from:

Odoni DI*, Tamayo-Ramos JA*, Sloothaak J, van Heck RGA, Martins dos Santos VAP, de Graaff LH, Suarez-Diez M, Schaap PJ. (2017) Comparative proteomics of Rhizopus delemar ATCC 20344 unravels the role of amino acid catabolism in fumarate accumulation. In PeerJ (https://doi.org/10.7717/peerj.3133).

*equal contributions 


\subsection{Abstract}

The filamentous fungus Rhizopus delemar naturally accumulates relatively high amounts of fumarate. Although the culture conditions that increase fumarate yields are well established, the network underlying the accumulation of fumarate is not yet fully understood. We set out to increase the knowledge about fumarate accumulation in $R$. delemar. To this end, we combined a transcriptomics and proteomics approach to identify key metabolic pathways involved in fumarate production in $R$. delemar, and propose that a substantial part of the fumarate accumulated in $R$. delemar during nitrogen starvation results from the urea cycle due to amino acid catabolism. 


\subsection{Introduction}

Fumarate, a dicarboxylic acid, is an important building block chemical for a number of high-value chemicals and materials. Amongst the microorganisms identified to be natural fumarate producers, the filamentous fungus Rhizopus delemar has the highest product yields (Foster and Waksman, 1939a). The most important factor influencing fumarate production in $R$. delemar is a high carbon:nitrogen ratio; extracellular fumarate accumulation happens after the growth phase, and especially when the nitrogen in the medium has been depleted (Goldberg et al., 2006; Magnuson and Lasure, 2004). The choice of nitrogen source has been reported to influence the final fumarate yield (Foster and Waksman, 1939a; Rhodes et al., 1959; Zhang et al., 2007), but so far no consensus on these influences has been reached. Another important factor influencing fumarate production in $R$. delemar is oxygen availability (Foster and Waksman, 1939a; Foster et al., 1949). Under fumarate producing conditions, $R$. delemar forms ethanol and other undesired fermentation by-products (Wright et al., 1996), directing carbon away from fumarate (Figure 2.1). Higher oxygen levels limit the amount of ethanol produced, and thus lead to higher fumarate yields. Fumarate production by fermentation has been extensively reviewed (Engel et al., 2008; Straathof and van Gulik, 2012).

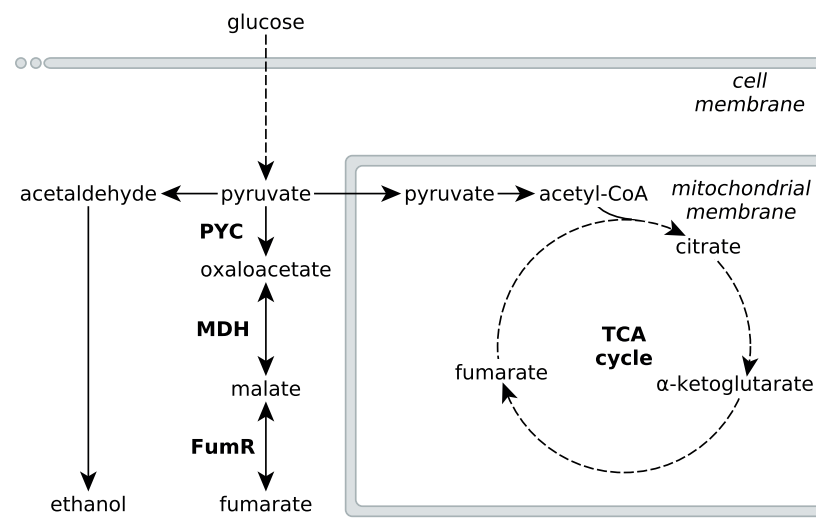

FIGURE 2.1. Metabolic pathways involved in fumarate metabolism in $\boldsymbol{R}$. delemar. Metabolic flux of $R$. delemar is predominantly directed towards fumarate (under aerobic conditions) or ethanol (under anaerobic conditions). The enzymes of the reductive TCA cycle are indicated in the scheme: PYC, pyruvate carboxylase; MDH, L-malate dehydrogenase and FumR, fumarase.

Although the culture conditions that increase fumarate accumulation in $R$. 
delemar are well established, natural product titers still cannot compete with chemical fumarate synthesis. To increase the amount of fumarate produced, $R$. delemar has been genetically modified (Meussen et al., 2012; Zhang et al., 2012; Zhang and Yang, 2012), but the occurrence of an ancestral whole-genome duplication as well as more recent gene- duplication events complicate the genetic engineering of $R$. delemar (Ma et al., 2009). A more promising approach for biological fumarate production would thus be rewiring the metabolism of a genetically more amenable cell-factory, based on $R$. delemar fumarate synthesis pathways.

Metabolic engineering approaches to increase fumarate production in microbial cell-factories that do not naturally accumulate high amounts of fumarate would greatly benefit from an in-depth understanding of the underlying metabolic pathways that affect the accumulation of fumarate in the natural fumarate producer $R$. delemar, as well as possible causes for this accumulation. Fumarate can be found as an intermediate in various different metabolic subsystems, and a number of pathways have been investigated for fumarate production in several microbial cell-factories such as Saccharomyces cerevisiae, Torulopsis glabrata, Scheffersomyces stipitis and Escherichia coli (Chen et al., 2015, 2016; Li et al., 2014; Song et al., 2013; Wei et al., 2015; Xu et al., 2013, 2012a; Zhang et al., 2015). Despite the large number of pathways leading to fumarate, the current consensus is that the reductive route of the TCA cycle in the cytosol is responsible for fumarate accumulation in $R$. delemar (Kenealy et al., 1986; Osmani and Scrutton, 1985; Overman and Romano, 1969; Peleg et al., 1989; Romano et al., 1967). The reductive TCA cycle comprises pyruvate carboxylase (PYC), L-malate dehydrogenase $(\mathrm{MDH})$ and fumarase (FumR), in which pyruvate is consecutively converted to oxaloacetate, L-malate and fumarate (Figure 2.1).

A controversial aspect of this pathway model is FumR. While overexpression of pyc and $m d h$ gave the expected increase of fumarate in $R$. delemar and Saccharomyces cerevisiae (Xu et al., 2012b; Zhang et al., 2012), overexpression of fumR in $R$. delemar as well as the introduction of fumR in $S$. cerevisiae and A. niger was reported to result in more L-malate rather than the accumulation of fumarate (De Jongh and Nielsen, 2008; Xu et al., 2012b; Zhang and Yang, 2012). There has been debate about the role of FumR in fumarate accumulation, discussed by Meussen et al. (2012). In summary, the reaction kinetics of FumR favour the conversion of fumarate to L-malate rather than the reverse direction, and FumR activity of acid-producing mycelium is completely blocked in the presence of 2 $\mathrm{mM}$ fumarate (note that the data on FumR inhibition have never been published, but that this finding is cited by Goldberg et al. (2006); Meussen et al. (2012). This 
suggests the presence of an alternative pathway that is responsible for fumarate accumulation in $R$. delemar.

In this study, we aim to provide a holistic overview of the pathways involved in fumarate accumulation in the natural fumarate producer $R$. delemar. To this end, we cultured the $R$. delemar strain ATCC 20344 under nitrogen starvation conditions, and varied oxygen availability to induce high (aerobic) and low (anaerobic) fumarate production. Combining transcriptomic and proteomic data obtained from the two conditions, we revealed the relationship between nitrogen metabolism and fumarate accumulation in $R$. delemar, mediated by the urea cycle.

\subsection{Materials and methods}

\subsubsection{Strains, media and culture conditions}

We selected the $R$. delemar strain ATCC 20344 (a kind gift from Adrie J.J. Straathof, Delft University of Technology) to study fumarate production. Note that $R$. delemar is more commonly known as $R$. oryzae (also Rhizopus nigricans and Rhizopus arrhizus) (Abe et al., 2007). Depending on the organic acid produced when grown on D-glucose, it is divided into two phylogenetically distinct types: type I strains, which produce primarily L-lactate, and type II strains, which produce mainly fumarate and L-malate (Abe et al., 2003). Complete medium agar plates containing $0.3 \%(\mathrm{w} / \mathrm{v})$ yeast extract, $0.3 \%(\mathrm{w} / \mathrm{v})$ malt extract, $0.3 \%$ $(\mathrm{w} / \mathrm{v})$ peptone, $2 \%(\mathrm{w} / \mathrm{v})$ glycerol, and $2 \%(\mathrm{w} / \mathrm{v})$ agar were used to generate spores. Mycelial biomass was produced using pre-culture medium containing $1 \%(\mathrm{w} / \mathrm{v}) \mathrm{D}$ glucose, $0.21 \%(\mathrm{w} / \mathrm{v})$ urea, $0.06 \%(\mathrm{w} / \mathrm{v}) \mathrm{KH}_{2} \mathrm{PO}_{4}, 0.05 \%(\mathrm{w} / \mathrm{v}) \mathrm{MgSO}_{4} \cdot 7 \mathrm{H}_{2} \mathrm{O}$ and $0.0018 \%(\mathrm{w} / \mathrm{v}) \mathrm{ZnSO}_{4} \cdot 7 \mathrm{H}_{2} \mathrm{O}$. Approximately $10^{6}$ spores $/ \mathrm{mL}$ were inoculated in 1L Erlenmeyer flasks containing $500 \mathrm{~mL}$ of pre culture medium and cultivations were carried out at $35{ }^{\circ} \mathrm{C}$ and $250 \mathrm{rpm}$ for $24 \mathrm{~h}$. The mycelium obtained was washed with demineralized water and transferred ( $\approx 25 \mathrm{~g}$ of wet biomass) to production medium, which contained $10 \%$ (w/v) D-glucose, $0.06 \%(\mathrm{w} / \mathrm{v}) \mathrm{KH}_{2} \mathrm{PO}_{4}$, $0.05 \%$ (w/v) $\mathrm{MgSO}_{4} \cdot 7 \mathrm{H}_{2} \mathrm{O}, 0.0018 \%(\mathrm{w} / \mathrm{v}) \mathrm{ZnSO}_{4} \cdot 7 \mathrm{H}_{2} \mathrm{O}$, and $1 \%(\mathrm{w} / \mathrm{v}) \mathrm{CaCO}_{3}$ (used as a neutralizing agent). Batch fermentations were performed at $35{ }^{\circ} \mathrm{C}$ and $600 \mathrm{rpm}$ in $2.5 \mathrm{~L}$ fermentors (Applikon, Schiedam, The Netherlands), with a working volume of $1.75 \mathrm{~L}$. Antifoam 204 was added to each fermentor ( $85 \mu \mathrm{L}$ ). The fermentation medium was aerated with $1.0 \mathrm{~L} / \mathrm{L}$ min, either with filtered air or $\mathrm{N}_{2}$ gas. 


\subsubsection{Metabolite analysis using HPLC}

Extracellular metabolite concentrations were determined by high-performance liquid chromatography (HPLC). A Thermo Accela HPLC, equipped with a Shodex KC-811 column, and coupled to a refractive index detector (Spectrasystem RI-150, sample frequency $5.00032 \mathrm{~Hz}$ ) and a UV-VIS detector (Spectrasytem UV1000, $\lambda=210 \mathrm{~nm}$ ), was used. Separations were performed by isocratic elution with $0.01 \mathrm{~N} \mathrm{H}_{2} \mathrm{SO}_{4}$ at a flow rate of $0.8 \mathrm{~mL} / \mathrm{min}$. Crotonic acid $(6 \mathrm{mM})$ was used as an internal standard.

\subsubsection{RNA isolation and quality control}

Frozen mycelium ( $\approx 100 \mathrm{mg}$ ) of $R$. delemar ATCC 20344 was submerged in $1 \mathrm{~mL}$ of Trizol reagent in a $2 \mathrm{~mL}$ tube, prefilled with a mix of glass beads with the following diameters: $1 \mathrm{~mm}(0.25 \mathrm{~g}), 0.1 \mathrm{~mm}(0.37 \mathrm{~g}), 5 \mathrm{~mm}$ (1 bead). Mycelium samples were disrupted using a FastPrep-24 Instrument (MP). After disruption, $200 \mu \mathrm{L}$ of chloroform were added and the mix was homogenated for $10 \mathrm{~s}$. The mix was poured into phase-lock gel tubes $(2 \mathrm{~mL})$, and centrifuged at maximum speed in a table-top centrifuge. The RNA present in the water phase was purified using the RNeasy Mini Kit (Qiagen), following the manufacturer's instructions. RNA integrity was assessed with an Experion system (Bio-Rad), and only high quality samples (RIN value $\geqslant 8$ ) were selected for whole transcriptome shotgun sequencing.

\subsubsection{RNA sequencing and quality check}

Illumina RNA sequencing (RNA seq) using the Casava pipeline version 1.8.2 and subsequent quality analysis of the FASTQ sequence reads was performed by BaseClear (Leiden, The Netherlands). The number of reads obtained was 20'539'199 for the aerobic and 24'519'028 for the anaerobic condition, with an average quality score (Phred) of 37.59 and 37.91, respectively. The raw data has been submitted to the European Nucleotide Archive (ENA), and can be found under the accession number PRJEB14210 (http://www.ebi.ac.uk/ena/data/view/PRJEB14210).

\subsubsection{RNA seq data processing}

Following the workflow suggested and validated by Davids et al. (2016), the RNA seq reads were filtered using SortMeRNA v1.9 (Kopylova et al., 2012), cutadapt v1.2.1 (Martin, 2011) and PRINSEQ v0.20.2 (Schmieder and Edwards, 2011). De novo assembly of the reads that passed the quality filtering was performed using 
the IDBA-UD assembler v1.1 (Peng et al., 2012). Read mapping and transcript coverage calculations were performed using Bowtie2 v2.2.2 (Langmead and Salzberg, 2012) and BEDTools (Quinlan and Hall, 2010). Note that in contrast to the proteomics analysis, which was performed on both biological replicates, only one biological replicate per condition was sent for RNA sequencing. The average nucleotide coverage is thus an indication of the transcript levels of a given transcript at the time of sampling, not the average of two biological replicates. A more extensive description of the RNA seq data processing, including example commands for every tool used, is given in (DOI: 10.7717/peerj.3133/supp-1).

\subsubsection{Preparation of cell free extracts for proteomic analysis}

$R$. delemar ATCC 20344 mycelium samples (2-3 g, press-dried), were washed with an ice-cold $20 \mathrm{mM}$ HEPES buffer $\mathrm{pH} 7.6$, containing $150 \mathrm{mM} \mathrm{NaCl}$, and resuspended in the same solution containing $1 \%(\mathrm{v} / \mathrm{v})$ protease inhibitor cocktail for yeast and fungi (Sigma). Mycelium suspensions were immediately disrupted using a French press (8'000 psi). Cell free extracts were centrifuged for 5 min at low speed (500 g), in order to remove unbroken cells and pellet debris. The remaining supernantants were further processed for LC-MS/MS analysis.

\subsubsection{Sample preparation for LC-MS/MS}

The protein content of the $R$. delemar ATCC 20344 cell free extracts was determined using the BCA protein assay (Thermo Fisher). Membrane-bound proteins were solubilised by mixing volumes of each sample, containing $25 \mu \mathrm{g}$ of protein, with equal volumes of a $2 \times$ solution of $20 \mathrm{mM}$ HEPES pH 7.6, containing $1 \mathrm{M}$ 6 -aminocaproic acid and $10 \mathrm{~g} / \mathrm{L}$ of n-dodecyl-beta-D-maltoside. Cell free extractdetergent mixes were incubated in a thermoblock for $1 \mathrm{~h}$ at $20^{\circ} \mathrm{C}$ and vigorous stirring (1'000 rpm). Afterwards, samples were sonicated in a water bath for 15 min, and finally they were centrifuged at $222^{\prime} 000 \mathrm{~g}$, in a benchtop centrifuge, for $30 \mathrm{~min}$. Obtained supernatants were subsequently concentrated using Microcon YM-10 columns (cutoff, 10 kDa; Millipore, Eschborn, Germany).

Samples from each biological replicate and culture condition were loaded into a $12 \%$ SDS-polyacrylamide gel, which was run until the loaded samples entered the gel. The gel was stained according to the manufacturer's instructions using Page Blue staining (Fermentas) and rinsed with ultrapure water. Each sample-gel lane was cut into one slice (approx. $1 \mathrm{~cm}^{2}$ ), carefully sliced into smaller pieces of about $1 \mathrm{~mm}^{3}$ and transferred into microcentrifuge tubes. Samples were destained and equilibrated through three washing steps using 
the following solutions: $50 \mathrm{mM}$ ammonium bicarbonate (ABC) (incubated $5 \mathrm{~min}$ ), ABC/acetonitrile (1:1, v/v) (incubated $5 \mathrm{~min}$ ) and neat acetonitrile (incubated $5 \mathrm{~min}$ ). These washing steps were repeated two times. The gel samples were then swelled in $10 \mathrm{mM}$ dithiothreitol (DTT) for $20 \mathrm{~min}$ at $56{ }^{\circ} \mathrm{C}$ to reduce protein disulfide bonds. Subsequently, the DTT solutions were removed and samples were alkylated with $50 \mathrm{mM}$ 2-chloroacetamide in $\mathrm{ABC}$, for $20 \mathrm{~min}$, at room temperature, in the dark. The 2 - chloroacetamide solutions were removed, and samples were again washed twice with: neat acetonitrile (incubated $5 \mathrm{~min}$ ), ABC (incubated $5 \mathrm{~min}$ ) and neat acetonitrile (incubated $5 \mathrm{~min}$ ). Approximately 150 $\mu \mathrm{L}$ of digestion buffer, containing sequencing grade modified trypsin $(12.5 \mathrm{ng} / \mu \mathrm{L})$ (Promega) in ABC, was added to each sample, making sure that all gel pieces were kept wet during digestion (adding, if necessary, additional ABC solution).

Protein samples were digested overnight at $37^{\circ} \mathrm{C}$. Peptide digestion products were extracted by adding $50 \mu \mathrm{L}$ of $2 \%$ trifluoroacetic acid (TFA), followed by an incubation step in a thermoblock for $20 \mathrm{~min}$, at room temperature and vigorous stirring (1'400 rpm). Gel pieces were then subjected to 20 s sonication in a water bath, centrifuged and supernatants were transferred to new tubes. The peptide extraction step was then repeated once by washing the gel pieces with buffer B ( $80 \%$ acetonitrile, $0.1 \%$ formic acid) followed by the mentioned incubation and sonication steps. Supernatants from both extractions were pooled and samples were placed in a vacuum centrifuge for acetonitrile evaporation util 20-40 $\mu \mathrm{L}$ were left. Finally, samples were acidified by addition of TFA $(1: 1, \mathrm{v} / \mathrm{v})$ and peptide clean-up procedure, prior to LC-MS/MS analysis, was performed using the "STop And Go Extraction" procedure as described before (Rappsilber et al., 2003).

\subsubsection{Mass spectrometric measurements and proteomic data analysis}

LC-MS/MS analysis was performed at the Radboud Proteomics Centre as described previously (Rajala et al., 2015). Measurements were performed by nanoflow reversed-phase C18 liquid chromatography (EASY nLC, Thermo Scientific) coupled online to a 7 Tesla linear ion trap Fourier-Transform ion cyclotron resonance mass spectrometer (LTQ FT Ultra, Thermo Scientific). The LC-MS/MS spectra obtained were identified and quantified using the maxQuant software (Cox and Mann, 2008). The peptides were mapped against the in silico proteomes of $R$. delemar ATCC 20344 (obtained from the transcriptomics experiment) and RA 99-880 (obtained from Genbank, Project ID: 13066 (Ma et al., 2009)) with the default settings, described in Sloothaak et al. (2015). Only proteins with 2 or more 
unique peptide hits were considered for further analysis. The mass spectrometry proteomics data have been deposited to the ProteomeXchange Consortium via the PRIDE (Vizcaíno et al., 2015) partner repository with the dataset identifier PXD004600.

\subsubsection{Metabolic pathway enrichment analysis}

Metabolic enzymes were annotated using PRIAM (Claudel-Renard et al., 2003), and subsequently assigned to KEGG (Kanehisa and Goto, 2000; Kanehisa et al., 2016) pathways (see (DOI: 10.7717/peerj.3133/supp-1) for details on the KEGG pathway mapping). Enrichment analysis of differentially expressed pathways was performed using the hypergeometric test implementation ("phyper") of the $R$ software environment (Ihaka and Gentleman, 1996). We used the identified proteins that could be mapped to a KEGG pathway as the universe (with size $\mathrm{N}$ $=277$ ). Note that the terms "differentially expressed" and "overexpressed" refer to differences in relative protein abundances, and denote a fold-change of 1.5 and 2 as lenient and stringent thresholds.

\subsection{Results and discussion}

\subsubsection{Fumarate and ethanol production of ATCC 20344 grown under aerobic and anaerobic conditions}

We chose to work with $R$. delemar ATCC 20344, henceforth referred to as ATCC 20344 , for its ability to produce fumarate in high quantities (Cao et al., 1996). ATCC 20344 was grown in batch fermentations under nitrogen starved conditions. The fumarate production rate was controlled by either supplying filtered air to the culture medium (aerobic condition), or restricting the amount of oxygen by flushing the system with $\mathrm{N}_{2}$ (anaerobic condition). D-glucose, fumarate and ethanol concentrations in the supernatant were measured via High Performance Liquid Chromatography (HPLC) to determine the time point with the largest difference in fumarate yield. The HPLC analysis showed comparable D-glucose consumption rates in the two conditions (Figure 2.2), with an average of 0.15 $\pm 0.03 \mathrm{~g} / \mathrm{h}$ and $0.11 \pm 0.03 \mathrm{~g} / \mathrm{h}$ for the aerobic and anaerobic condition, respectively. Fumarate production was higher in the aerobic condition, whereas in the anaerobic condition ethanol production prevailed. Note that Lin and Wang (1991) showed that Rhizopus spp grow very poorly under absolute anaerobic conditions, but that most of the tested strains grew "quite well" under microaerobic conditions. The use of silicone tubing on our fermentors, which are slightly oxygen 
permeable even when flushed with pure nitrogen gas (Weusthuis et al., 1994), allowed ATCC 20344 to utilise glucose at the same rate as under aerobic conditions, while keeping fumarate production to a minimum. However, the amount of oxygen entering through the silicone tubing was below the detection limit of the probes measuring dissolved oxygen in the fermentors, and we refer to the two conditions as "aerobic" and "anaerobic" rather than "aerobic" and "microaerobic".

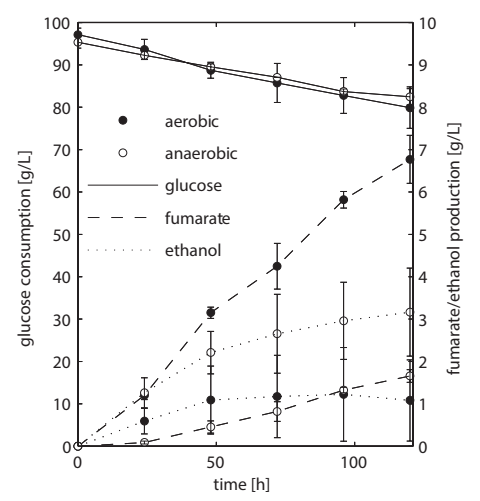

FIGURE 2.2. HPLC analysis of fermentation broth of $\boldsymbol{R}$. delemar ATCC 20344 grown under aerobic and anaerobic conditions. Total D-glucose consumption and fumarate and ethanol production of $R$. delemar ATCC 20344. The measurement points show the average of two biological replicates.

The fumarate yields (gram per gram substrate D-glucose consumed) are summarised in Table 2.1. The maximum fumarate yield $(0.41 \pm 0.06 \mathrm{~g} / \mathrm{g})$ in the aerobic condition was observed after $96 \mathrm{~h}$ of fermentation. A comparable yield $(0.35 \pm 0.05 \mathrm{~g} / \mathrm{g})$ was already observed after $24 \mathrm{~h}$ of fermentation. In contrast, the fumarate yield in the anaerobic condition increased continuously at a slow pace, being highest after $120 \mathrm{~h}$ of fermentation $(0.13 \pm 0.02 \mathrm{~g} / \mathrm{g})$. Thus, the largest difference in fumarate yield between the two conditions was observed at the start of the experiment, and we chose $t=24 \mathrm{~h}$ as the time point for the transcriptome and proteome analyses.

\subsubsection{Transcriptome and proteome of ATCC 20344 under high and low fumarate producing conditions}

Enzyme activities, and thus metabolism, are affected by various factors such as post-translational modifications, allosteric control, and substrate availability. 
Table 2.1: Fumarate yields of ATCC 20344 grown under aerobic and anaerobic conditions.

\begin{tabular}{rcc}
\hline time [h] & \multicolumn{2}{c}{ Fumarate yield $\pm \mathrm{sd}$ [g/g D-glucose] } \\
\cline { 2 - 3 } & aerobic & anaerobic \\
\hline 24 & $0.349 \pm 0.055$ & $0.029 \pm 0.015$ \\
48 & $0.375 \pm 0.001$ & $0.077 \pm 0.015$ \\
72 & $0.380 \pm 0.055$ & $0.098 \pm 0.012$ \\
96 & $0.411 \pm 0.063$ & $0.115 \pm 0.014$ \\
120 & $0.397 \pm 0.044$ & $0.132 \pm 0.022$ \\
\hline
\end{tabular}

Metabolic fluxes can therefore not be inferred directly from protein abundances. Nevertheless, contrasting enzyme abundance levels between the high and low fumarate producing condition (ATCC 20344 snapshot proteomes) indicate differences in the metabolic state of ATCC 20344 at the time point of sampling. To determine differential protein abundances via LC-MS/MS, a reference proteome database is required for peptide mass fingerprinting. To date, $R$. delemar RA 99-880, henceforth referred to as RA 99-880, is the only fully sequenced $R$. delemar strain of which also the proteome is publicly available (Ma et al., 2009). However, if the RA 99-880 reference proteome is used as only reference database, conservative amino acid substitutions in ATCC 20344 will reduce the sensitivity, as protein identification relies on an exact peptide mass. To provide a complete database of the metabolic potential as well as an overview of the metabolic state of ATCC 20344 under high and low fumarate producing conditions, we combined transcriptomic and proteomic data of ATCC 20344 grown under high and low fumarate producing conditions. The transcriptome was used to construct a database of the ATCC 20344 in silico proteome, and the relative protein abundances were obtained by mapping the peptides from the snapshot proteomes against both the ATCC 20344 and RA 99-880 in silico proteomes, the latter to account for possible errors in the de novo transcript assembly. The experimental setup is outlined in Figure 2.3.

The RNA seq reads obtained from the aerobic and anaerobic conditions were combined into one dataset, and assembled de novo, resulting in 13'531 contigs (DOI: 10.7717/peerj.3133/supp-2). We used PRIAM (Claudel-Renard et al., 2003) to assign EC numbers to the six-frame translation products of the de novo contigs, as well as to the RA 99-880 reference proteome (DOI: 10.7717/peerj.3133/supp-3). In ATCC 20344, we predicted 1'283 metabolic enzymes, covering 529 EC numbers. In RA 99-880, we predicted 1'389 metabolic enzymes, covering 504 EC numbers. 


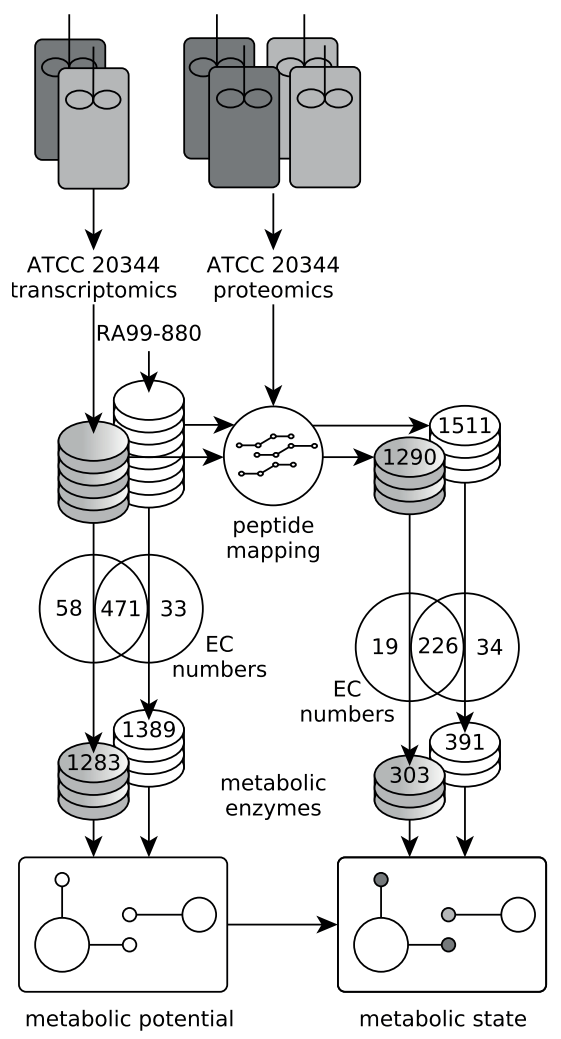

FiguRE 2.3. Experimental setup. Workflow to establish the metabolic potential and metabolic state of ATCC 20344 grown under high and low fumarate producing conditions. The metabolic enzymes predicted in the ATCC 20344 and RA 99-880 in silico proteomes provide a map of the metabolic potential, while the metabolic enzymes identified in the proteomics experiment were used to determine the metabolic state of ATCC 20344 under high and low fumarate producing conditions.

The metabolic enzymes were mapped to KEGG pathway maps (Kanehisa and Goto, 2000; Kanehisa et al., 2016) in order to obtain a rough estimate of the metabolic potential of $R$. delemar.

The proteins obtained from the aerobic and anaerobic conditions were subjected to a shotgun proteomics analysis. A total of 1'290 and 1'511 proteins were identified in the ATCC 20344 and RA 99-880 proteomes, respectively. Roughly one third of the identified proteins comprised metabolic enzymes. A list of all EC numbers predicted in the ATCC 20344 and RA 99-880 in silico proteomes, as well as the relative protein abundances and average nucleotide coverages of the proteins 
and transcripts identified in the experimental conditions (resulting in a total of 1'970 proteins, although some are duplicates due to combining ATCC 20344 and RA 99-880 in one file), can be found in (DOI: 10.7717/peerj.3133/supp-4). The protein pathway coverage (number of ECs per pathway covered by proteins with the predicted function in ATCC 20344 and RA 99-880) is given in (DOI: 10.7717/peerj.3133/supp-5).

\subsubsection{Metabolic pathway enrichment analysis}

To determine which pathways play an important role in fumarate accumulation in ATCC 20344, we obtained a list of enzymatic proteins that directly consume or produce fumarate according to the KEGG database (Kanehisa and Goto, 2000; Kanehisa et al., 2016), and analysed their presence and abundance in the ATCC 20344 high and low fumarate producing conditions (Table 2.2). In addition, we performed pathway enrichment analysis of differentially expressed enzymes (Table 2.3 and (DOI: 10.7717/peerj.3133/supp-5)).

Table 2.2: List of enzymes involved in fumarate metabolism with their respective protein abundances under high and low fumarate producing conditions.

\begin{tabular}{|c|c|c|c|c|c|}
\hline \multirow{2}{*}{$\begin{array}{l}\text { EC } \\
\text { number }\end{array}$} & \multirow{2}{*}{$\begin{array}{l}\text { Consensus } \\
\text { protein } \\
\text { identifier }^{a}\end{array}$} & \multicolumn{2}{|c|}{ Relative protein abundance $\pm \mathrm{sd}[\%]$} & \multirow{2}{*}{$\begin{array}{l}\log _{2} \mathrm{FC} \\
\text { aerobic / } \\
\text { anaerobic }\end{array}$} & \multirow[t]{2}{*}{ Enzyme name } \\
\hline & & aerobic & anaerobic & & \\
\hline 4.2 .1 .2 & Rd_01690 & $1.51 \pm 0.16$ & $0.66 \pm 0.06$ & 1.21 & $\begin{array}{l}\text { Fumarate hydratase } \\
\text { (fumarase, FumR) }\end{array}$ \\
\hline 4.3.2.1 & Rd_00962 & $0.09 \pm 0.01$ & $0.06 \pm 0.02$ & 0.60 & $\begin{array}{l}\text { Argininosuccinate } \\
\text { lyase (ASL) }\end{array}$ \\
\hline 1.3 .98 .1 & Rd_00873 & $0.04 \pm 4 e^{-3}$ & $0.01 \pm 2 e^{-3}$ & 1.34 & $\begin{array}{l}\text { Dihydroorotate } \\
\text { dehydrogenase }\end{array}$ \\
\hline 3.7.1.2 & Rd_01207 & $0.03 \pm 3 e^{-3}$ & 0.00 & - & $\begin{array}{l}\text { Fumaryl- } \\
\text { acetoacetase }\end{array}$ \\
\hline 1.3.5.1 & Rd_01783 & $0.01 \pm 2 e^{-3}$ & 0.00 & - & $\begin{array}{l}\text { Succinate } \\
\text { dehydrogenase }\end{array}$ \\
\hline 4.3.2.2 & Rd_00964 & $5 e^{-3} \pm 7 e^{-4}$ & $0.01 \pm 8 e^{-7}$ & -1.59 & $\begin{array}{l}\text { Adenylosuccinate } \\
\text { lyase }\end{array}$ \\
\hline
\end{tabular}

We found that, amongst the enzymes which interact directly with fumarate and were identified in the ATCC 20344 snapshot proteomes, FumR (EC 4.2.1.2), is the most highly abundant enzyme, both under high and low fumarate producing conditions (Table 2.2). Although there is no clear enrichment of the TCA cycle enzymes among the differentially expressed metabolic proteins (Table 2.3), the three enzymes of the reductive TCA cycle, PYC (EC 6.4.1.1, relative protein abundance [\% a.u.]: aerobic $=2.14 \pm 0.31$, anaerobic $=0.40 \pm 0.02, \log _{2} \mathrm{FC}$ (aerobic/anaerobic) = 2.44), $\mathrm{MDH}$ (3 isozymes with EC 1.1.1.37, relative protein abundance [\% a.u.]: 
aerobic $=0.48 \pm 0.06 ; 0.15 \pm 0.06 ; 2.43 \pm 0.08$, anaerobic $=0.10 \pm 0.01 ; 0.02 \pm$ $0.00 ; 1.85 \pm 0.09, \log _{2} \mathrm{FC}$ (aerobic/anaerobic) $\left.=2.20 ; 3.19 ; 0.40\right)$, and FumR (EC 4.2.1.2, see Table 2.2 for relative protein abundance), are all overexpressed in the high fumarate producing condition.

The second most highly abundant protein related to fumarate metabolism is argininosuccinate lyase (ASL) (EC 4.3.2.1). ASL is a urea cycle enzyme involved in arginine biosynthesis. The arginine biosynthesis pathway showed a significant number of differentially expressed proteins (Table 2.3), and we found that the enzymes comprising the urea cycle are overexpressed in the high fumarate producing condition (Table 2.4). This suggests that in ATCC 20344, the urea cycle plays an important role in fumarate accumulation. Most interestingly, ASL and FUM constitute a crucial link between carbon and nitrogen metabolism by connecting the TCA- and urea cycles (also referred to as "Krebs bicycle"). 


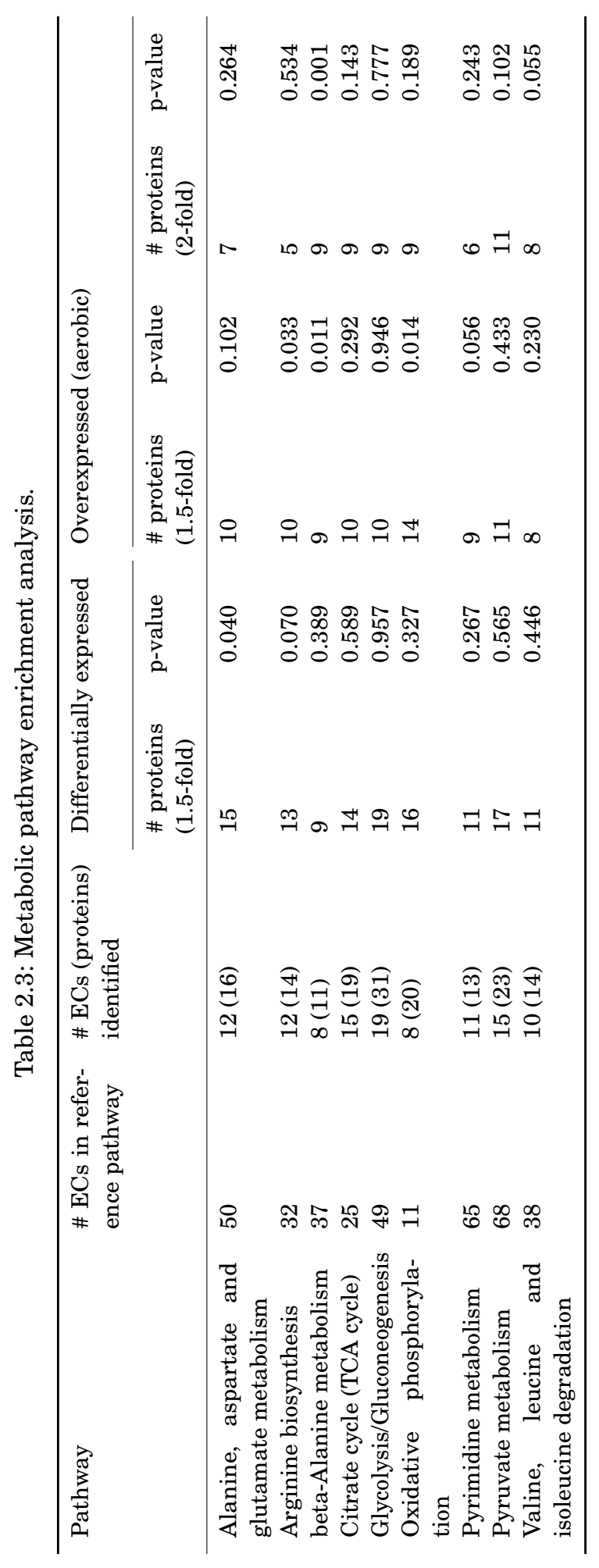


Table 2.4: List of enzymes involved in fumarate metabolism with their respective protein abundances under high and low fumarate producing conditions.

\begin{tabular}{|c|c|c|c|c|c|}
\hline \multirow{2}{*}{$\begin{array}{l}\text { EC } \\
\text { number }\end{array}$} & \multirow{2}{*}{$\begin{array}{l}\text { Consensus } \\
\text { protein }^{a} \\
\text { identifier }^{a}\end{array}$} & \multicolumn{2}{|c|}{ Relative protein abundance \pm sd [\%] } & \multirow{2}{*}{$\begin{array}{l}\log _{2} \mathrm{FC} \\
\text { aerobic / } \\
\text { anaerobic }\end{array}$} & \multirow[t]{2}{*}{ Enzyme name } \\
\hline & & aerobic & anaerobic & & \\
\hline 2.1 .3 .3 & Rd_01058 (A) & $0.03 \pm 0.01$ & $0.02 \pm 8 e^{-3}$ & 0.51 & $\begin{array}{l}\text { Ornithine carbamoyl- } \\
\text { transferase (OTC) }\end{array}$ \\
\hline 6.3.4.5 & Rd_01708 (R) & $0.86 \pm 0.07$ & $0.57 \pm 0.05$ & 0.59 & $\begin{array}{l}\text { Argininosuccinate syn- } \\
\text { thase (ASS) }\end{array}$ \\
\hline 6.3.4.5 & Rd_01709 (R) & 0.00 & $0.04 \pm 0.02$ & - & $\begin{array}{l}\text { Argininosuccinate syn- } \\
\text { thase (ASS) }\end{array}$ \\
\hline 4.3.2.1 & Rd_00962 (A) & $0.09 \pm 0.01$ & $0.06 \pm 0.02$ & 0.60 & $\begin{array}{l}\text { Argininosuccinate lyase } \\
\text { (ASL) }\end{array}$ \\
\hline 3.5.3.1 & Rd_00988 (R) & $0.13 \pm 0.02$ & $0.07 \pm 0.01$ & 0.97 & Arginase (ARG) \\
\hline 3.5.3.1 & Rd_00989 (R) & $0.19 \pm 0.02$ & $0.11 \pm 0.03$ & 0.76 & Arginase (ARG) \\
\hline
\end{tabular}

${ }^{a}$ Identifiers refer to IDs in (DOI: 10.7717/peerj.3133/supp-4). Note that, where possible, ATCC 20344 enzymes were prioritised. In this case, all enzymes were identified in the ATCC 20344 proteome.

The observed protein abundances for FumR and ASL offer an explanation for the importance of a high carbon:nitrogen ratio for fumarate accumulation in Rhizopus spp (Goldberg et al., 2006; Magnuson and Lasure, 2004). In humans, starvation induces a net breakdown of stored energy sources, starting with fatty acids and, when exposed to prolonged starving conditions, proteins from muscle tissue (Felig, 1973). The degradation of protein, or amino acids, results in the liberation of ammonia, which is then carried to the urea cycle as L-glutamate (Feillet and Leonard, 1998), 1998). In the urea cycle, the L-glutamate is converted to urea, which is subsequently excreted (Feillet and Leonard, 1998). Based on the significant enrichment scores of pathways involved in amino acid metabolism (Table 2.3), we propose that the nitrogen starvation, induced by the transfer of ATCC 20344 from growth- to production medium, triggers a similar switch in metabolism, and amino acid catabolism starts to occur. The resulting fluxes through the urea cycle yield an excess of fumarate (Figure 2.4).

Specifically, the pathway for the degradation of the branched-chain amino acids (BCAA) valine, leucine and isoleucine shows a significant number of overexpressed enzymes in the high fumarate producing condition. BCAA catabolism is initiated by BCAA aminotransferase (EC 2.6.1.42), which catalyses the transfer of an amino group from any of the three BCAAs to $\alpha$-ketoglutarate, yielding L-glutamate and the respective $\alpha$-keto acid as products (Figure 2.4). Under starvation conditions in which both the nitrogen and carbon source in the culture medium are limited, the carbon skeletons of the deaminated amino acids can be used to replenish acetyl-CoA (from leucine), or the TCA cycle intermediate succinyl-CoA (from valine) or both of these metabolites (from isoleucine), and thereby, ultimately, to generate energy for survival. Under conditions of excess 


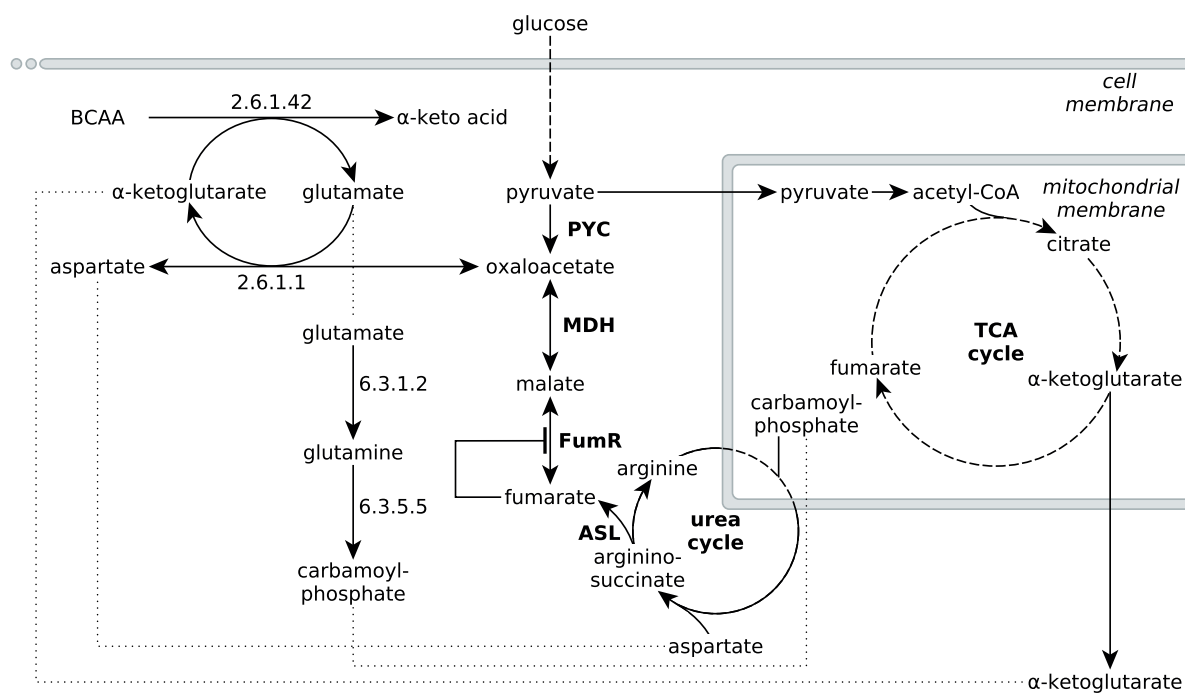

FIGURE 2.4. Extended network of metabolic pathways involved in fumarate metabolism in $\boldsymbol{R}$. delemar. The extended model of fumarate accumulation reconstructed from the ATCC 20344 snapshot proteomes under high and low fumarate producing conditions takes the formation of fumarate via the urea cycle into account.

carbon and limited nitrogen, however, it is unlikely that amino acid catabolism is driven by energy demand of the organism. More importantly, it is crucial for $R$. delemar grown under nitrogen depleted conditions to decouple carbon catabolism from cell proliferation, as there is little nitrogen available for de novo protein biosynthesis.

One way to decouple carbon catabolism from biomass formation is by reducing the amount of ATP generated. Under aerobic growth conditions, ATP is generated via oxidative phosphorylation. Under anaerobic conditions, $R$. delemar generates ATP via ethanol fermentation (Figure 2.1). Another option for ATP generation is alternative respiration, mediated by the key enzyme alternative oxidase (AOX). AOX diverts the electrons passing through the electron transport chain in the mitochondria at the ubiquinone pool and transfers them directly to oxygen, thereby bypassing the oxidative phosphorylation complexes III and IV, resulting in an overall lower ATP yield. Gu et al. (2014) found that the activity of AOX is positively correlated with fumarate production in $R$. delemar. In contrast, we identified AOX (Rd_00967 (A)) only in the snapshot proteome of the anaerobic condition. The transcriptomics measurements further underpin our proteomics 
results, since aox was overexpressed ( $>4$-fold) in the anaerobic condition (DOI: 10.7717/peerj.3133/supp-4). This might seem counterintuitive at first, since the expression of aox is generally regarded as a means of dealing with increased oxidative stress. However, the electron flow through AOX has been found to be inversely proportional to nitrogen availability in various different plant systems (Vanlerberghe, 2013). The increase of AOX under nitrogen limited conditions and the resulting decrease of the respiratory ATP yield have been associated with a deliberately reduced efficiency in converting carbon to biomass; by using the non-energy conserving AOX, the "redundant" carbohydrate can be metabolised without being coupled to growth.

Another way of decoupling carbon catabolism from energy generation, and thereby cell proliferation, is channelling the products from amino acid degradation to the mitochondria for mitochondrial protein synthesis, and thereby away from cytosolic protein synthesis; a mechanism suggested to take place under nitrogen starvation conditions in human cells, irrespective of the D-glucose availability (Johnson et al., 2014). In this, amino acid catabolism is the first step to adapt to nitrogen limiting conditions, and we propose that, in a similar mechanism, the urea cycle plays a key role for the accumulation of fumarate in $R$. delemar. This is supported by the work of Chen et al. (2015) who showed that, from a range of selected enzymes, overexpressing ASL, while keeping the expression of adenylosuccinate lyase low, resulted in the highest fumarate titer in Torulopsis glabrata.

\subsection{Conclusions}

The accumulation of fumarate in the natural fumarate producer $R$. delemar has been mostly attributed to the consecutive conversion of pyruvate to oxaloacetate, L-malate and fumarate by cytosolic enzymes of the reductive TCA cycle. In addition, our proteomics data have revealed that the nitrogen-limitation under fumarate producing conditions induces amino acid catabolism, which leads to an increased flux through the urea cycle. Further investigation is required to verify the involvement of the urea cycle in $R$. delemar fumarate accumulation. As $R$. delemar can utilise urea as nitrogen source, higher fluxes through the urea cycle will not necessarily lead to a measurable increase of urea in the medium. Our comparative proteomics analysis of high and low fumarate producing conditions in $R$. delemar ATCC 20344 has resulted in a novel holistic view on fumarate production that expands the knowledge on fumarate production in this fungus, and provides a basis for further biochemical explorations regarding biotechnological 
fumarate production.

\subsection{Acknowledgements}

We would like to thank Sybe Hartmans, Michael Volpers and Brendan Ryback for critically reading and commenting on draft versions of the manuscript. In addition, we would like to thank Bastian Hornung for valuable input for the RNA seq data analysis, Ruud Weusthuis for valuable feedback on general aspects of this manuscript, and Tom Schonewille and Merlijn van Gaal for their contribution in the experimental part of this work.

\section{Data availability}

Proteomics data:

PRIDE, http://www.ebi.ac.uk/pride/archive/projects/PXD004600

RNA seq data:

EMBL-EBI (PRJEB14210), http://www.ebi.ac.uk/ena/data/view/PRJEB14210

\section{Supplementary information}

Supplementary files for this chapter can be found online at:

http://dx.doi.org/10.7717/peerj.3133\#supplemental-information 



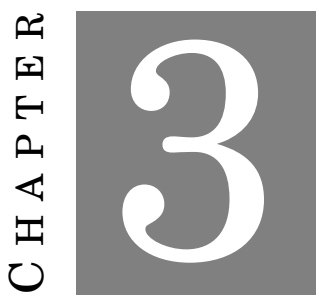

Aspergillus niger SECRETES CITRATE TO INCREASE IRON BIOAVAILABILITY

This chapter is adapted from:

Odoni DI, van Gaal MP, Schonewille T, Tamayo-Ramos JA, Martins dos Santos VAP, Suarez-Diez M, Schaap PJ. (2017) Aspergillus niger secretes citrate to increase iron bioavailability. In Frontiers in Microbiology

(https://doi.org/10.3389/fmicb.2017.01424). 


\subsection{Abstract}

Aspergillus niger has an innate ability to secrete various organic acids, including citrate. The conditions required for $A$. niger citrate overproduction are well described, but the physiological reasons underlying extracellular citrate accumulation are not yet fully understood. One of the less understood culture conditions is the requirement of growth-limiting iron concentrations. While this has been attributed to iron-dependent citrate metabolising enzymes, this straightforward relationship does not always hold true. Here, we show that an increase in citrate secretion under iron limited conditions is a physiological response consistent with a role of citrate as $A$. niger iron siderophore. We found that $A$. niger citrate secretion increases with decreasing amounts of iron added to the culture medium and, in contrast to previous findings, this response is independent of the nitrogen source. Differential transcriptomics analyses of the two A. niger mutants NW305 (gluconate non-producer) and NW186 (gluconate and oxalate non-producer) revealed up-regulation of the citrate biosynthesis gene citA under iron limited conditions compared to iron replete conditions. In addition, we show that $A$. niger can utilise Fe(III) citrate as iron source. Finally, we discuss our findings in the general context of the $\mathrm{pH}$-dependency of $A$. niger organic acid production, offering an explanation, besides competition, for why $A$. niger organic acid production is a sequential process influenced by the external $\mathrm{pH}$ of the culture medium. 


\subsection{Introduction}

The filamentous fungus Aspergillus niger has an innate ability to secrete organic acids in high quantities, and is essential as a biotechnological citrate producer (Schuster et al., 2002). On glucose as carbon source, wild type A. niger secretes gluconate and oxalate as well as citrate. To inhibit by-production of gluconate and oxalate, $A$. niger citrate production requires $\mathrm{pH} \leq 2.5$ and the absence of manganese $\left(\mathrm{Mn}^{2+}\right)$ ions, thereby enforcing overproduction of citrate instead (Currie, 1917; Kubicek and Röhr, 1977; Ruijter et al., 1999). Further production conditions that have been reported to influence external citrate accumulation are, amongst others, the choice and concentrations of the carbon and nitrogen sources, and the concentrations of trace elements in the culture medium (Karaffa and Kubicek, 2003).

Although suggested to be the basis of industrial $A$. niger citrate production (Neilands, 1981), the physiological reason underlying the particular requirement of suboptimal iron concentrations to prompt increased $A$. niger citrate secretion is not yet fully understood. Iron ( $\mathrm{Fe}$ ) is essential for virtually all biological systems. In aerobic environments, ferrous ( $\mathrm{Fe}(\mathrm{II})$ ) iron is oxidised to ferric (Fe(III)) oxyhydroxide polymers $(\mathrm{FeOOH})$, which are stable and have low solubility in aqueous environments, especially at neutral pH (Guerinot and Yi, 1994). Thus, although omnipresent, iron is often biologically unavailable. Iron excess, on the other hand, can be harmful due to the ability of $\mathrm{Fe}$ (II) to catalyse the formation of cell-damaging reactive oxygen species, and microbes have thus developed complex systems for tight control over iron uptake and intracellular storage (Haas, 2014).

In fungi, there are four known systems of iron uptake: i) low-affinity (ferrous) iron uptake, ii) heme uptake and degradation, iii) reductive iron assimilation (RIA), and iv) siderophore mediated (ferric) iron uptake (which, in some cases, involves RIA) (Haas, 2014). Low affinity ferrous iron uptake is less relevant under iron limited conditions, and the $A$. niger heme uptake system has been recently studied (Franken et al., 2014). Iron siderophores are low molecular weight molecules that are secreted to scavenge (ferric) iron from the environment and make it available to the microbe. This can be either by reduction of the siderophore bound Fe(III) to more soluble Fe(II), which is then imported separately from the siderophore (i.e. RIA), or by uptake of the whole iron-siderophore complex (Neilands, 1995).

Iron uptake and siderophore biosynthesis in Aspergilli has been found to be under the control of the transcription factors SreA and HapX, which are interconnected in a negative feedback loop (Hortschansky et al., 2007; Schrettl 
et al., 2008). Under conditions of iron excess, SreA represses HapX as well as the high-affinity iron uptake system and iron siderophore biosynthesis, thereby avoiding the uptake of toxic amounts of iron (Schrettl et al., 2008). Under iron limited conditions, HapX is derepressed, and, in turn, HapX represses SreA as well as iron-dependent pathways (Hortschansky et al., 2007).

With the exception of some budding and fission yeasts, fungi synthesise their own iron siderophores (Haas, 2014). Coprogen B and ferrichrome were identified as two A. niger iron siderophores (Franken et al., 2014). However, oxalate and citrate both have inherent chelating properties (Dutton and Evans, 1996; Gadd, 1999), and citrate is an established iron siderophore in various different plant and prokaryotic systems (Cox, 1980; Cox et al., 1970; Frost and Rosenberg, 1973; Guerinot et al., 1990; Silva et al., 2009). Therefore, we hypothesise that, besides coprogen B and ferrichrome, citrate could serve as additional A. niger iron siderophore.

In this study, we aim to further elucidate the link between iron limitation and increased $A$. niger citrate secretion. To this end, we measured citrate per glucose production of $A$. niger grown with varying iron concentrations and nitrogen sources, and established a direct link between citrate secretion and iron availability. We investigated the effect of iron limitation on the A. niger transcriptome, and found changes associated to biomass, iron siderophore, and citrate and oxalate biosynthesis genes. Finally, we found that $A$. niger can utilise Fe(III) citrate as iron source. Our results support the hypothesis that citrate acts as $A$. niger iron siderophore, and provide insights on why $A$. niger organic acid production is a $\mathrm{pH}$-dependent process.

\subsection{Materials and methods}

\subsubsection{Strains, media and culture conditions}

The A. niger strains N402 (cspA1), NW305 (cspA, goxC17, $\Delta a r g B$ ) (Ruijter et al., 2003), and NW186 (cspA1, goxC17, prtF28, $\Delta \arg B, \operatorname{pyr} A 6)$, a $\triangle \arg B$ and pyrA6 derivative of NW185 (Ruijter et al., 1999), were used for this study. For the growth experiments, $A$. niger NW186 was transformed as described (Kusters-van Someren et al., 1991) with the plasmid pGW635 (Goosen et al., 1989), carrying the pyrA gene of $A$. niger. This complements the pyrA6 transformation marker and restores the uridine prototrophy. In addition, we measured citrate production under low iron stress (no iron added to the medium, see Supplementary file 1) in A. niger NW129 (cspA1, goxC17, pyrA1) (Ruijter et al., 1997a). 
To obtain spores, $A$. niger was grown (from glycerol stock), for 4 days, on complete medium (CM) agar plates, containing, per 1'000 mL: $2 \mathrm{~g}$ meat peptone, $1 \mathrm{~g}$ yeast extract, $1 \mathrm{~g}$ casamino acids, $0.3 \mathrm{~g}$ yeast ribonucleic acids, $15 \mathrm{~g}$ agar and minimal medium (MM) salts (MM salts, per 1'000 mL: $6 \mathrm{~g} \mathrm{NaNO}$, $1.5 \mathrm{~g}$ $\mathrm{KH}_{2} \mathrm{PO}_{4}, 0.5 \mathrm{~g} \mathrm{KCl}$ and $0.5 \mathrm{~g} \mathrm{MgSO}_{4} \cdot 7 \mathrm{H}_{2} \mathrm{O}$ ), added before sterilisation, and $1 \mathrm{~mL}$ vitamin solution (composition of vitamin solution, per $100 \mathrm{~mL}: 0.01 \mathrm{~g}$ thiamine, $0.10 \mathrm{~g}$ riboflavin-5P, $0.01 \mathrm{~g}$ p-aminobenzoic acid, $0.10 \mathrm{~g}$ nicotinamide, $0.05 \mathrm{~g}$ pyridoxine-HCl, $0.01 \mathrm{~g}$ pantothenic acid, $0.002 \mathrm{~g}$ biotin), $1 \mathrm{~mL}$ Vishniac (Vishniac and Santer, 1957) solution, $50 \mathrm{mM}(9 \mathrm{~g})$ glucose, and $0.02 \%$ arginine, added after sterilisation. After 4 days of growth, spores were harvested with $12-13 \mathrm{~mL}$ of Saline-Tween solution.

A total of $10^{6}$ spores/mL were inoculated in $1 \mathrm{~L}$ Erlenmeyer flasks containing $200 \mathrm{~mL}$ of $A$. niger production medium (PM, per 1'000 mL: $1.2 \mathrm{~g} \mathrm{NaNO}_{3}$ or $0.93 \mathrm{~g}$ $\left.\left(\mathrm{NH}_{4}\right)_{2} \mathrm{SO}_{4}, 0.5 \mathrm{~g} \mathrm{KH}_{2} \mathrm{PO}_{4}, 0.2 \mathrm{~g} \mathrm{MgSO}_{4} \cdot 7 \mathrm{H}_{2} \mathrm{O}\right), 100 \mathrm{mM}(\approx 20 \mathrm{~g} / \mathrm{L})$ glucose, $40 \mu \mathrm{L}$ adjusted Vishniac (Vishniac and Santer, 1957) solution (with, per 1'000 mL: either $1.0 \mathrm{~g} \mathrm{(+)}$ or $10 \mathrm{~g} \mathrm{(++)} \mathrm{FeSO}_{4} \cdot 7 \mathrm{H}_{2} \mathrm{O}$, or $0.94 \mathrm{~g} \mathrm{(+)} \mathrm{or} 9.75 \mathrm{~g} \mathrm{(++)} \mathrm{C}_{6} \mathrm{H}_{5} \mathrm{FeO}_{7} \cdot \mathrm{H}_{2} \mathrm{O}$ (Fe(III) citrate), or no Fe (-)), and $0.02 \%$ arginine (NW305, NW186) supplement. Note that, for a better understanding of the system, yeast extract was not used in any of the experiments. No measures were taken to keep the medium completely free from trace amounts of iron. The conditions referred to as -Fe describe a condition in which no iron was added to the medium, and shows the response of A. niger to low iron stress rather than the response of this fungus to complete absence of iron.

Time course experiments were performed in duplicates in a shake flask incubator at $30{ }^{\circ} \mathrm{C}$ and $200 \mathrm{rpm}$. Supernatant samples were taken every $24 \mathrm{~h}$. Mycelial dry weight was measured after $96 \mathrm{~h}$.

The yeast strains Cyberlindnera jadinii DSM 2361, Cyberlindnera fabianii CBS 5640, Hanseniaspora uvarum CECT 11105, Kluyveromyces lactis CBS 739, Saccharomyces cerevisiae NCYC 2826, Wickerhamomyces anomalus DSM 6766 and Wickerhamomyces ciferii CBS 111 were grown (from glycerol stock) on yeast extract peptone dextrose plates (YPD, per 1'000 mL: $10 \mathrm{~g}$ yeast extract, $20 \mathrm{~g}$ peptone, $20 \mathrm{~g}$ glucose and $15 \mathrm{~g}$ agar) and, per 1'000 mL: $0.15 \mathrm{~g}$ uracil, $0.5 \mathrm{~g}$ leucine, $0.075 \mathrm{~g}$ tryptophan and $12.5 \mathrm{~g}$ histidine supplements. From these plates, overnight cultures were grown in $10 \mathrm{~mL}$ liquid YPD medium with the appropriate supplements (see below). The cultures were spun down, washed with sterile demi water, and resuspended in $10 \mathrm{~mL}$ demi water. $100 \mu \mathrm{L}$ of the resuspension was used to inoculate $100 \mathrm{~mL}$ Erlenmeyer flasks (in duplicate) containing $20 \mathrm{~mL}$ of medium (per 1'000 mL: $20 \mathrm{~g}$ glucose, $5 \mathrm{~g}\left(\mathrm{NH}_{4}\right)_{2} \mathrm{SO}_{4}, 3 \mathrm{~g} \mathrm{KH}_{2} \mathrm{PO}_{4}$ and $0.5 \mathrm{~g}$ 
$\mathrm{MgSO}_{4} \cdot 7 \mathrm{H}_{2} \mathrm{O}$, the same supplements mentioned before, and $40 \mu \mathrm{L}$ Verduyns (Verduyn et al., 1992) trace metal solution, either with or without $\mathrm{FeSO}_{4} \cdot 7 \mathrm{H}_{2} \mathrm{O}$, or $40 \mu \mathrm{L}$ Verduyns (Verduyn et al., 1992) trace metal solution without $\mathrm{FeSO}_{4} \cdot 7 \mathrm{H}_{2} \mathrm{O}$, but $0.12 \mathrm{mg} / \mathrm{L} \mathrm{C}_{6} \mathrm{H}_{5} \mathrm{FeO}_{7} \cdot \mathrm{H}_{2} \mathrm{O}$ (Fe(III) citrate).

\subsubsection{Metabolite analysis using HPLC}

Extracellular metabolite concentrations were determined by high-performance liquid chromatography (HPLC). An ICS5000 HPLC (Thermo Scientific), equipped with an Aminex HPX-87H column (BioRad) at $60{ }^{\circ} \mathrm{C}$, and coupled to a refractive index detector detector (Shodex RI-101, sample frequency $5 \mathrm{~Hz}$ ) and a Thermo UV/VIS detector $(\lambda=210 \mathrm{~nm}$ ), was used. Separations were performed by elution with $0.016 \mathrm{~N} \mathrm{H}_{2} \mathrm{SO}_{4}$ at a flow rate of $0.6 \mathrm{~mL} / \mathrm{min}$. An organic acid standard, containing oxalic acid, citric acid, malic acid, succinic acid and itaconic acid, and a separate glucose standard, with $2 \mathrm{mM}, 5 \mathrm{mM}, 10 \mathrm{mM}, 20 \mathrm{mM}$ (both organic acid standard and glucose standard), $100 \mathrm{mM}$ and $200 \mathrm{mM}$ (only glucose standard) were used to calculate calibration curves for quantification of the extracellular metabolite concentrations. Measurement of known concentrations of a Fe(III) citrate standard matched the areas under the peak for the normal citrate standard. Propionic acid $(6 \mathrm{mM})$ was used as an internal standard.

\subsubsection{RNA isolation and quality control}

RNA extraction was performed using the Maxwell 16 LEV simplyRNA Tissue kit (Promega). Frozen mycelium $(\approx 100 \mathrm{mg}$ ) of the sample was submerged in $400 \mu \mathrm{L}$ Homogenising buffer supplemented with $8 \mu \mathrm{L}$ 1-thioglycerol in a $2 \mathrm{~mL}$ Lysing matrix $\mathrm{C}$ tube (MP), prefilled with a mix of glass beads. Mycelium samples were disrupted using a FastPrep-24 instrument (MP). After disruption all liquid was transferred to a LEV RNA Cartridge. $200 \mu \mathrm{L}$ lysis buffer was added and the rest of the extraction was performed by a Maxwell MDx AS3000 machine (Promega) following the protocol. RNA integrity and quantity were assessed with an Experion system (Bio-Rad), and only high quality samples (RIN value $\geq 7$ ) were selected. Total RNA was sent directly to BaseClear (Leiden, The Netherlands) for whole transcriptome shotgun sequencing.

\subsubsection{RNA sequencing and quality check}

RNA sequencing (RNA seq) and initial quality check was performed by BaseClear, and was reported as follows: "Single-end sequence reads were generated using 
the Illumina HiSeq2500 system. FASTQ sequence files were generated using the Illumina Casava pipeline version 1.8.3. Initial quality assessment was based on data passing the Illumina Chastity filtering. Subsequently, reads containing adapters and/or PhiX control signal were removed using an in-house filtering protocol. The second quality assessment was based on the remaining reads using the FASTQC quality control tool version 0.10.0."

\subsubsection{RNA seq data processing}

The RNA seq reads were filtered using SortMeRNA v1.9 (Kopylova et al., 2012) and Trimmomatic v0.32 (Bolger et al., 2014). Read mapping against A. niger ATCC 1015 (Andersen et al., 2011; Nordberg et al., 2013) was performed using STAR v2.5.0c (Dobin et al., 2013). Gene coverage calculations were performed using BEDTools v2.17.0 (Quinlan and Hall, 2010) and subsequently normalised for the respective library sizes. Differential expression analysis was performed using the $R$ package edgeR (Robinson et al., 2010). RNA seq normalisation and differential expression was performed simultaneously for each comparison. Genes with a count per million $(\mathrm{CPM}) \geq 1$ in at least two samples were considered to be expressed and kept for further analysis. Trimmed mean of M-values normalisation was performed as implemented in the $\mathrm{R}$ package edgeR. $\mathrm{P}$-values were corrected for multiple testing using the Benjamini-Hochberg procedure. The terms "differentially expressed" and "overexpressed" refer to differences in read counts per CDS, and denote a fold-change $\geq 1.5$ (FDR $\leq 0.05$ ). A detailed pipeline of the RNA seq data processing can be found in Supplementary file 2 . The aligned .bam files have been submitted to the European Nucleotide Archive (ENA), and can be found under the accession number PRJEB20746.

\subsubsection{Enrichment analyses}

The protein products of the expressed genes were annotated using PRIAM (Claudel-Renard et al., 2003), and subsequently assigned to KEGG pathway maps (Kanehisa and Goto, 2000; Kanehisa et al., 2016). Metabolic pathway enrichment analysis was performed using the hypergeometric test implementation ("phyper") of the R software environment (Ihaka and Gentleman, 1996).

Protein localisation prediction was performed with the Softberry protComp tool (www.softberry.com). Enrichment analysis was performed on differentially expressed genes per organelle as described above. 


\subsection{Results}

When grown in a culture medium with glucose as sole carbon source, wild type A. niger strains secrete multiple organic acids (Figure 3.1). Under moderate acidic conditions ( $\mathrm{pH}$ 5), mainly oxalic acid is secreted. Additionally, glucose is converted extracellularly to gluconate by a secreted glucose oxidase. Upon further acidification of the medium to a $\mathrm{pH}$ below 2.5 , citrate becomes the predominantly secreted organic acid (Ruijter et al., 1999). To remove gluconate and oxalate as confounding factors, but without having to exert control over the external $\mathrm{pH}$ and thereby influencing iron availability, we worked with $A$. niger strain NW186. This strain bears two mutations, one leading to a frameshift in the gene encoding glucose oxidase (gox 17 ), and a nonsense mutation in the gene encoding oxaloacetate hydrolase (oahA), making this strain a gluconate and oxalate non-producer (Han et al., 2007; Ruijter et al., 1999).

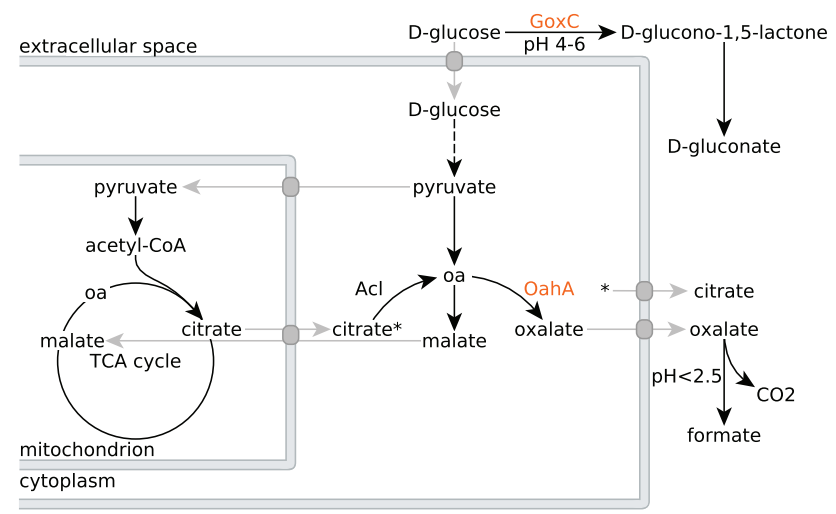

FiguRE 3.1. Organic acid production in A. niger N402 and its derivatives. Metabolic routes for citrate, oxalate and gluconate production in A. niger $\mathrm{N} 402$ and its derivatives (oa = oxaloacetate). Mutants defective in GoxC can no longer produce gluconate, whereas mutants defective in OahA can no longer produce oxalate. A hypothetical malate/citrate antiport (Karaffa and Kubicek, 2003) is depicted in grey.

Another confounding factor is that iron, zinc, copper, manganese, phosphorus, magnesium, potassium and nitrogen limitation reportedly all have a stimulating effect on A. niger organic acid production (Chesters and Rolinson, 1951; Kubicek and Röhr, 1977). Thus, we grew NW186 with different nitrogen sources, and varying amounts of iron sulphate $\left(\mathrm{Fe}(\mathrm{II}) \mathrm{SO}_{4}\right)$ added to the medium, but otherwise identical growth conditions (see Materials and methods). A. niger biomass produc- 
tion increases with the amount of iron added to the medium (Table 3.1) and, in contrast to previous findings (Currie, 1917), this happens irrespective of whether nitrogen is supplied as nitrate $\left(\mathrm{NaNO}_{3}\right)$ or ammonium $\left(\left(\mathrm{NH}_{4}\right)_{2} \mathrm{SO}_{4}\right)$. Note that no special measures were taken to keep the cultures completely free from any traces of iron, which will most likely be present. However, the increasing biomass upon addition of more iron to the medium (Table 3.1) shows that, in our experimental setup, iron is the growth-limiting factor; the observations described are thus a direct effect of the amount of $\mathrm{Fe}(\mathrm{II}) \mathrm{SO}_{4}$ added to the medium.

Table 3.1: Final biomass [g/L] of A. niger NW186 and the two control strains N402 and NW305 grown with different nitrogen sources and varying iron concentrations in the medium (Fe source: $\mathrm{Fe}(\mathrm{II}) \mathrm{SO}_{4}$ ).

\begin{tabular}{lcccccccc}
\hline \multirow{2}{*}{$\begin{array}{l}\text { Strain (major organic } \\
\text { acid(s) produced) }\end{array}$} & \multicolumn{3}{c}{$\mathrm{N}$ source: $\mathrm{NaNO}_{3}$} & & \multicolumn{3}{c}{$\mathrm{N}$ source: $\left(\mathrm{NH}_{4}\right)_{2} \mathrm{SO}_{4}$} \\
\cline { 2 - 3 } & $-\mathrm{Fe}$ & $+\mathrm{Fe}$ & $++\mathrm{Fe}$ & & $-\mathrm{Fe}$ & $+\mathrm{Fe}$ & $++\mathrm{Fe}$ \\
\hline N402 (gluconate, & & & & & & & & \\
oxalate, citrate) & $0.39 \pm 0.01$ & $0.66 \pm 1 e^{-3}$ & $1.52 \pm 3 e^{-3}$ & & $0.51 \pm 1 e^{-3}$ & $1.48 \pm 3 e^{-3}$ & $2.34 \pm 0.01$ \\
NW305 (oxalate, citrate) & $0.97 \pm 0.02$ & $1.08 \pm 0.04$ & $1.73 \pm 0.02$ & & $0.91 \pm 0.01$ & $1.97 \pm 0.02$ & $3.45 \pm 1 e^{-3}$ \\
NW186 (citrate) & $1.09 \pm 1 e^{-3}$ & $1.58 \pm 0.01$ & $1.84 \pm 1 e^{-3}$ & & $0.89 \pm 0.01$ & $2.17 \pm 0.03$ & $3.39 \pm 0.08$ \\
\hline
\end{tabular}

In contrast to the increase in $A$. niger biomass production (Table 3.1), and again irrespective of whether nitrogen is supplied as $\mathrm{NaNO}_{3}$ or $\left(\mathrm{NH}_{4}\right)_{2} \mathrm{SO}_{4}$, increasing the amount of $\mathrm{Fe}(\mathrm{II}) \mathrm{SO}_{4}$ decreases citrate per glucose production in NW186 (Figure 3.2A). In addition, we found that NW186 pre-grown without iron stopped secreting citrate after $\mathrm{Fe}(\mathrm{II}) \mathrm{SO}_{4}$ was added to the culture medium (Figure 3.2B). Final biomass reached $0.93 \pm 0.02 \mathrm{~g} / \mathrm{L}$ and $0.71 \pm 1 e^{-3} \mathrm{~g} / \mathrm{L}$ in the cultures grown with and without $\mathrm{Fe}(\mathrm{II}) \mathrm{SO}_{4}$ added to the medium, respectively.

Compared to NW186, decreasing the amount of iron added to the medium did not have an as equally pronounced effect on total citrate per glucose production in the two control strains N402 and NW305 (Figure 3.3). The direct relationship between iron limitation and citrate secretion is expected to be less straightforward in $A$. niger strains that are able to produce multiple organic acids in major quantities, and the shared relationship of iron on organic acid production is suggested by the secretion pattern of oxalate in NW305, where decreasing iron availability increases both oxalate and citrate per glucose production (Figure 3.3B). 

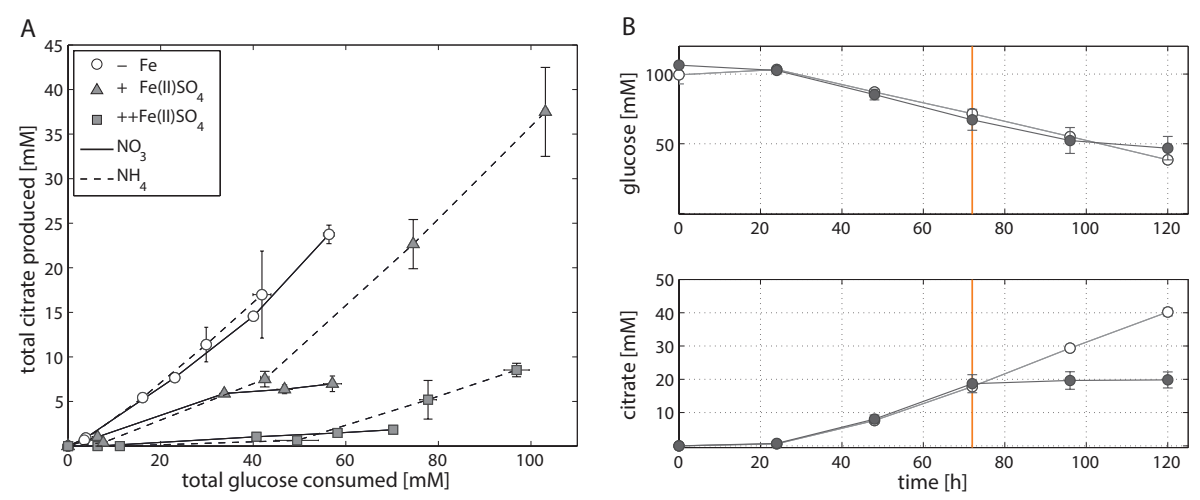

FIGURE 3.2. Iron-dependent citrate production of $A$. niger NW186.

(A) Total citrate production per glucose consumption of $A$. niger NW186, grown without addition of iron (empty circles), or varying amounts of $\mathrm{Fe}(\mathrm{II}) \mathrm{SO}_{4}$ (triangles = iron limited, squares = iron excess), and either $\mathrm{NaNO}_{3}$ (solid line) or $\left(\mathrm{NH}_{4}\right)_{2} \mathrm{SO}_{4}$ (dashed line) as nitrogen source. (B) Total glucose and citrate concentration of $A$. niger NW186 grown with (filled symbols) or without (empty symbols) $\mathrm{Fe}(\mathrm{II}) \mathrm{SO}_{4}$ added to the medium at $\mathrm{t}=72 \mathrm{~h}$ (orange line), and $\mathrm{NaNO}_{3}$ as nitrogen source. Measurement points were taken once every 24 $\mathrm{h}$ and show the average of two biological replicates. The error bars indicate the estimation of standard deviation divided by the number of replicates $(\mathrm{N})$ rather than $\mathrm{N}-1$.

\subsubsection{A. niger iron-dependent gene expression of biomass, iron siderophore and citrate biosynthesis genes}

The shared relationship of iron on both citrate and oxalate secretion in NW305 (Figure 3.3B), especially in comparison to the direct relationship of iron on citrate secretion in NW186 (Figure 2A) suggests that increased citrate secretion in NW186 is a result of impaired oxalate secretion due to the OahA mutation. This suggests a lesser role of citrate in wild-type strains of $A$. niger, especially before medium acidification to $\mathrm{pH} \leq 2.5$, where citrate starts to take over as primary organic acid secreted even in wild type strains (Currie, 1917). To gain further insights into the adaptations to iron limitation, especially with regard to the organic acid secreted, of the oxalate-impaired (hence citrate producing) NW186 mutant, we compared the transcriptional response of NW186 grown without iron added to the medium (NW186 -Fe) to the control strain NW305 grown without iron (NW305 -Fe) or excess iron (NW305 ++Fe) added to the medium (Figure 3.4).

The RNA seq reads obtained from the different conditions were mapped 

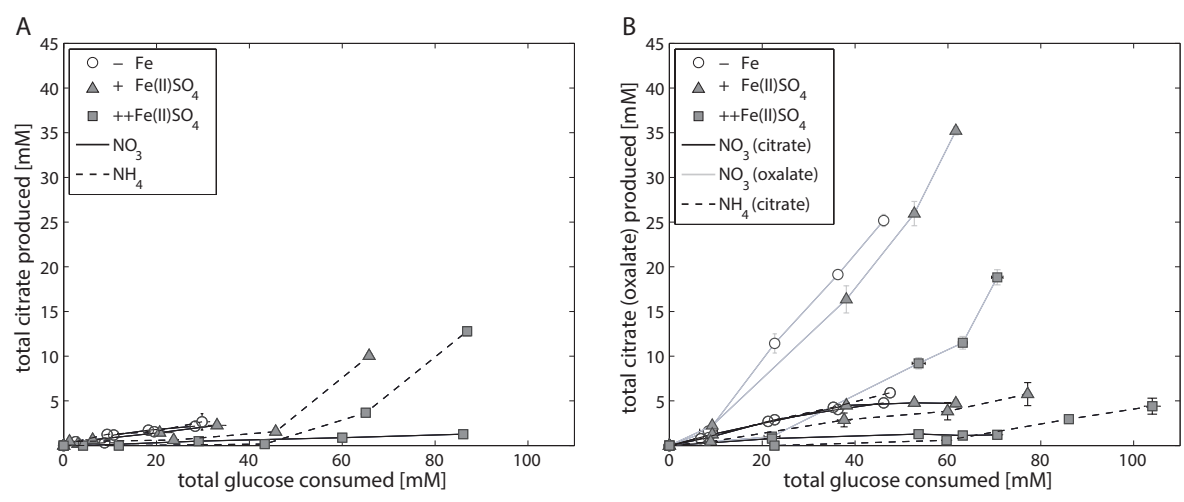

FIGURE 3.3. Iron-dependent citrate production of the control strains A. niger N402 and NW305. Total citrate production per glucose consumption of the control strains $A$. niger N402 (A) and $A$. niger NW305 (B), grown without addition of iron (empty symbols), or varying amounts of $\mathrm{Fe}(\mathrm{II}) \mathrm{SO}_{4}$ (triangles = iron limited, squares = iron excess), and either $\mathrm{NaNO}_{3}$ (solid line) or $\left(\mathrm{NH}_{4}\right)_{2} \mathrm{SO}_{4}$ (dashed line) as nitrogen source. Note that iron-dependent total oxalate per glucose production (grey, solid line) was plotted as example for one experiment $\left(\mathrm{NW} 305, \mathrm{~N}\right.$ source $\left.=\mathrm{NaNO}_{3}\right)$. Measurement points were taken once every $24 \mathrm{~h}$ and show the average of two biological replicates. The error bars indicate the estimation of standard deviation divided by the number of replicates $(\mathrm{N})$ rather than $\mathrm{N}-1$.

against the annotated $A$. niger ATCC 1015 genome (Andersen et al., 2011). Of the 11910 ATCC 1015 reference genes, reads were mapped (with a count per million $(\mathrm{CPM}) \geq 1)$ to 9239,8687 and 8815 genes in NW305 ++Fe, NW305 Fe and NW186 -Fe, respectively (Table 3.2). Of the genes expressed, 3332 were differentially expressed (FDR $\leq 0.05)$ between the control NW305 ++Fe $v$ s NW305 $-\mathrm{Fe}$, and 1252 were differentially expressed (FDR $\leq 0.05)$ between NW186 -Fe $v s$ NW305 -Fe (Table 3.2, Supplementary file 3).

Conforming the results presented in Table 3.1, metabolic pathway enrichment analysis (Supplementary file 4) showed that addition of iron to the A. niger culture medium has the strongest effect on biosynthesis pathways leading to biomass formation, i.e. starch and sucrose metabolism, and biosynthesis of various amino acids is up-regulated in iron replete $v s$ iron deplete conditions (i.e. in NW305 ++Fe compared to NW305-Fe). In addition, fatty acid biosynthesis showed enrichment of differentially expressed genes between NW305 ++Fe and NW305 -Fe. In comparison, the biggest difference between NW186 -Fe and NW305-Fe was 


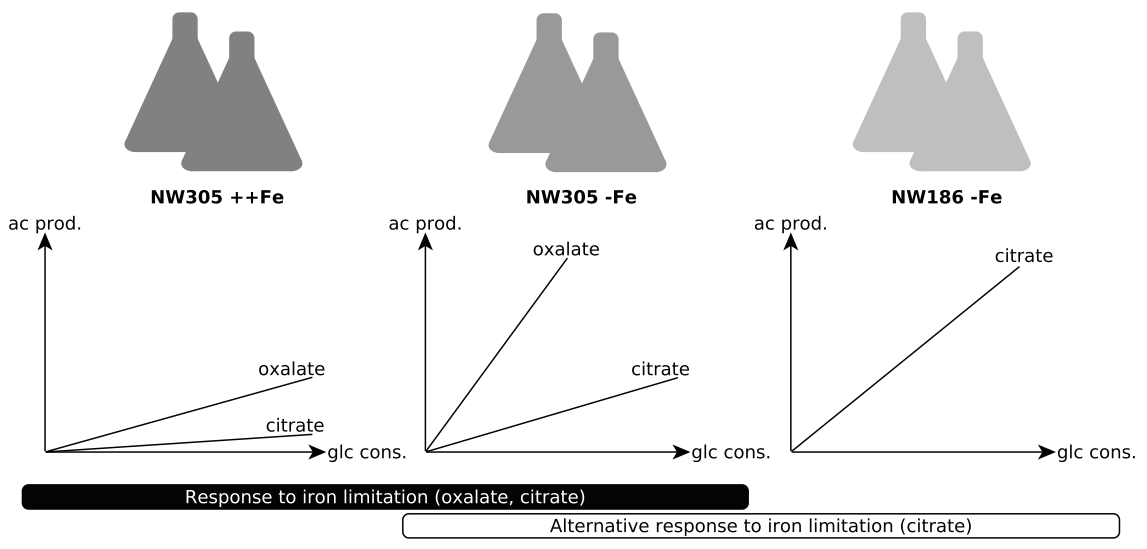

FIGURE 3.4. Conditions chosen for differential expression analyses. Experimental setup for RNA seq analyses. The control strain NW305 grown without iron added to the medium was used as reference, and compared to both NW305 grown with excess iron and NW186 grown without iron. The graphs depict the organic acid production profile of the 3 growth conditions. For the $A$. niger response to iron limitation, we compared the transcriptomic landscape of NW305 grown under conditions of iron excess, or without iron added to the medium. The transcriptional response of NW305 grown with or without iron was compared to NW186 grown without iron added to the medium.

related to lipoic and steroid biosynthesis, and drug and xenobiotics metabolism pathways.

We analysed expression levels of $A$. niger genes reported to be involved in iron homeostasis and siderophore biosynthesis (Franken et al., 2014; Haas, 2012). In agreement with (Franken et al., 2014; Haas, 2003), A. niger iron siderophore biosynthesis shows a clear response to iron limitation at transcriptional level (Figure 3.5, Supplementary file 5). Note that most of the proteins listed in Supplementary file 5 are based on best bi-directional Blast hits with the A. niger CBS 513.88 proteins identified by Franken et al. (2014). We included 1181156 (ATCC 1015 transId 1181432) in the list as possible transcriptional factor related to iron homeostasis and/or organic acid production due to its high expression level and pattern of transcription. 1181156 has a similar basic helix-loop-helix structure as the known Aspergillus fumigatus transcription factor SrbA, which was found to be essential for adaptation of this fungus to low iron stress (Blatzer et al., 2011). In addition, we included all ATCC 1015 proteins with putative 
Table 3.2: RNA seq mapping and differential expression analysis

\begin{tabular}{|c|c|c|c|}
\hline $\begin{array}{l}\text { Strain and } \\
\text { culture condition }\end{array}$ & $\begin{array}{l}\text { NW305 } \\
++\mathrm{Fe}\end{array}$ & $\begin{array}{l}\text { NW305 } \\
-\mathrm{Fe}\end{array}$ & $\begin{array}{l}\text { NW186 } \\
-\mathrm{Fe}\end{array}$ \\
\hline $\begin{array}{l}\text { \# reads after QC filtering } \\
\text { (see Supplementary file } 2 \text { ) }\end{array}$ & $\begin{array}{l}51^{\prime} 965 ' 960(1) \\
29 ' 101^{\prime} 217(2)\end{array}$ & $\begin{array}{l}45^{\prime} 2400^{\prime} 789(1) \\
52^{\prime} 325^{\prime} 240(2)\end{array}$ & $\begin{array}{l}43^{\prime} 227^{\prime} 405(1) \\
57^{\prime} 754^{\prime} 970(2)\end{array}$ \\
\hline $\begin{array}{l}\text { Uniquely mapped reads } \\
\text { (against ATCC1015 CDS) }\end{array}$ & $\begin{array}{l}57.96 \%(1) \\
61.22 \%(2)\end{array}$ & $\begin{array}{l}71.49 \%(1) \\
73.13 \%(2)\end{array}$ & $\begin{array}{l}68.82 \%(1) \\
72.70 \%(2)\end{array}$ \\
\hline \# genes expressed $(\mathrm{CPM} \geq 1)$ & $9^{\prime} 239$ & $8^{\prime} 687$ & $8^{\prime} 815$ \\
\hline $\begin{array}{l}\text { \# genes differentially expressed, } \\
\log _{2} \mathrm{FC} \text { threshold } \geq 0.58(\text { FDR } \leq 0.05)\end{array}$ & \multicolumn{3}{|c|}{$1 ’ 252$} \\
\hline $\begin{array}{l}\text { \# EC covered (mapped } \\
\text { to KEGG pathways) }\end{array}$ & \multicolumn{3}{|c|}{465} \\
\hline $\begin{array}{l}\text { \# EC differentially expressed, } \\
\log _{2} \mathrm{FC} \text { threshold } \geq 0.58(\mathrm{FDR} \leq 0.05)\end{array}$ & \multicolumn{3}{|c|}{196} \\
\hline
\end{tabular}

metalloreductase/ferric (chelate) reductase activity in the list (Supplementary file 5), and indicated which enzymes use iron as a co-factor (Supplementary file 3). In the ATCC 1015 in silico proteome, there are 163 enzymes that interact with iron as ligand according to the BRENDA database (Schomburg et al., 2004) of which 58 were differentially expressed (FDR $\leq 0.05$ ) between NW305 $++\mathrm{Fe}$ vs NW305-Fe, although there was no clear pattern of up- or down-regulation in response to the amount of iron added to the medium (i.e. 23 enzymes were up-regulated in NW305 ++Fe, and 32 were down-regulated; see Supplementary file 3).

Similar as for iron siderophore biosynthesis genes (Figure 3.5, Supplementary file 5), we found that both citrate synthase (citA, EC 2.3.3.1) and $o a h A$ (EC 3.7.1.1) were transcriptionally up-regulated in response to low iron stress (Table 3.3). In addition, ATP-citrate lyase (EC 2.3.3.8) and, to a lesser extent, $\left(\mathrm{NAD}^{+}\right)$isocitrate dehydrogenase were transcriptionally up-regulated in response to low iron stress (Table 3.3). In contrast, expression of citrate metabolising enzymes aconitase (EC 4.2.1.3) and $\left(\mathrm{NADP}^{+}\right)$isocitrate dehydrogenase (NADP-IDH, EC 1.1.1.42) did not show exclusively iron-dependent transcriptional regulation; most expressed isozymes were up-regulated in the reference NW305 -Fe compared to both NW305 $++\mathrm{Fe}$ and NW186 -Fe (Table 3.3).

Organelle specific differential expression enrichment analysis revealed the biggest difference between the 3 conditions was found in the plasma membrane (Table 3.4). Additionally, we observed enrichment of differentially expressed peroxisomal proteins in response to iron availability in the medium, i.e. in NW305 


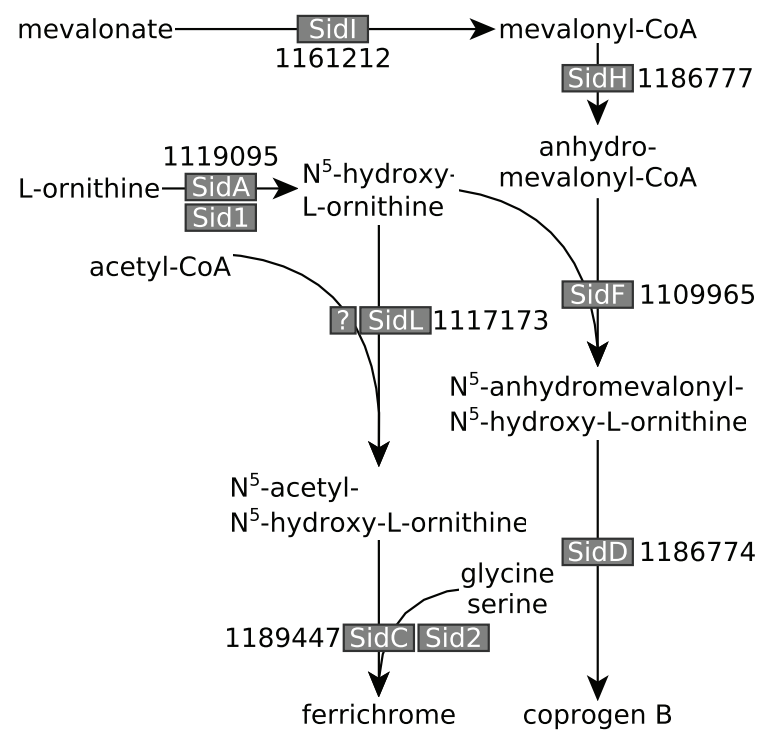

FIGURE 3.5. A. niger iron siderophore biosynthesis pathway. Iron siderophore biosynthesis genes identified in A. niger (adapted from Haas (2012) and Franken et al. (2014)). A. niger ATCC 1015 transcript identifiers are denoted next to the names of the enzymes given in other fungi. All genes, except the gene encoding the elusive A. niger SidL homologue, were overexpressed under iron limited conditions.

$++\mathrm{Fe}$ vs NW305 -Fe, but not in NW186 -Fe vs NW305 -Fe (Table 3.4). These differences could be attributed to the finding that many enzymes that participate in fungal iron siderophore biosynthesis are located in peroxisomes (Gründlinger et al., 2013). In addition, peroxisomes participate in metabolism of oxygen metabolites (Schrader and Fahimi, 2006). Under iron excess, low affinity ferrous iron uptake might lead to an excess of $\mathrm{Fe}$ (II) in the cell, which can catalyse the Fenton reaction: $\mathrm{Fe}(\mathrm{II})+\mathrm{H}_{2} \mathrm{O}_{2} \rightarrow \mathrm{Fe}(\mathrm{III})+\mathrm{OH}^{-}+{ }^{\circ} \mathrm{OH}$. The resulting oxygen radical $\mathrm{OH}$ can have cell-damaging effects (Schrader and Fahimi, 2006). However, if $\mathrm{H}_{2} \mathrm{O}_{2}$ can be decomposed to $\mathrm{O}_{2}$ and $\mathrm{H}_{2} \mathrm{O}$ by gluthatione-peroxidase (EC 1.11.1.9) or catalase (EC 1.11.1.6), the formation of $\mathrm{OH}$ can be prevented (Schrader and Fahimi, 2006). Although we could not identify an ATCC 1015 glutathione-peroxidase, there are 9 predicted catalases in the A. niger ATCC 1015 reference proteome (Supplementary file 3), of which 4 are predicted to be located in the peroxisome (1201726, not differentially expressed; 1116766, overexpressed in $++\mathrm{Fe} v s-\mathrm{Fe}$; 
Table 3.3: Differentially expressed enzymes involved in citrate and oxalate metabolism.

\begin{tabular}{|c|c|c|c|c|}
\hline $\begin{array}{l}\text { ATCC } 1015 \text { tran- } \\
\text { sriptId, proteinId }\end{array}$ & $\begin{array}{l}\text { Predicted EC } \\
\text { number }\end{array}$ & Enzyme name & $\begin{array}{ll}\log _{2} \mathrm{FC} & \mathrm{NW} 305 \\
++\mathrm{Fe} \text { vs } & \mathrm{NW} 305 \\
-\mathrm{Fe}(\mathrm{FDR}) & \end{array}$ & $\begin{array}{l}\log _{2} \mathrm{FC} \text { NW186 - } \\
\mathrm{Fe} \text { vs NW305-Fe } \\
\text { (FDR) }\end{array}$ \\
\hline 1141647,1141371 & 2.3.3.1 & citrate synthase & $-1.222(0.002)$ & $-0.167(0.949)$ \\
\hline 1218960, 1218684; & 4.2 .1 .3 & aconitase & $0.237(0.770)$ & $-0.984(0.124)$ \\
\hline 1148603, 1148327; & & & $-0.763(0.114)$ & $-0.984(0.055)$ \\
\hline 1181034,1180758 & & & $0.398(0.556)$ & $-0.841(0.079)$ \\
\hline 1148025,1147749 & 1.1.1.41 & $\begin{array}{l}\text { (NAD+) isocitrate } \\
\text { dehydrogenase }\end{array}$ & $-0.636(0.226)$ & $0.164(0.949)$ \\
\hline 1175666,1175390 & 1.1.1.42 & $\begin{array}{l}\text { (NADP+) isocitrate } \\
\text { dehydrogenase }\end{array}$ & $-2.033(0.000)$ & $-1.135(0.022)$ \\
\hline $\begin{array}{l}\text { 1111634, 1111358; } \\
1147138,1146862\end{array}$ & 2.3.3.8 & ATP-citrate lyase & $\begin{array}{l}-1.381(0.000) \\
-1.178(0.005)\end{array}$ & $\begin{array}{l}-0.026(0.994) \\
0.193(0.934)\end{array}$ \\
\hline 1145545,1145269 & 3.7.1.1 & $\begin{array}{l}\text { oxaloacetate acetyl- } \\
\text { hydrolase }\end{array}$ & $-1.427(0.000)$ & $0.446(0.732)$ \\
\hline
\end{tabular}

1119521, overexpressed in $++\mathrm{Fe} v s-\mathrm{Fe}$; 1158108, not differentially expressed), 3 are predicted to be cytosolic (1155727, not differentially expressed; 1137750 , underexpressed in $++\mathrm{Fe} v s$-Fe; 1228383, not differentially expressed) and one each mitochondrial (1181451, overexpressed in $++\mathrm{Fe} v s-\mathrm{Fe}$ ) or secreted (1204436, overexpressed in $++\mathrm{Fe} v s-\mathrm{Fe})$. Another peroxisomal enzyme that had an interesting expression pattern was pyruvate oxidoreductase (EC 1.2.7.1, transcriptId: 1162221, proteinId: 1161945), which was overexpressed in both NW186 -Fe and NW305 ++Fe compared to NW305 -Fe (Supplementary file 3).

Table 3.4: Organelle specific differential expression enrichment analysis

\begin{tabular}{lccc}
\hline Organelle & $\begin{array}{c}\text { \# genes expressed } \\
(\mathrm{CPM} \geq 1)\end{array}$ & $\begin{array}{c}\text { \# genes differentially expressed, } \log _{2} \mathrm{FC} \\
\text { threshold } \geq 0.58 \text { (FDR } \leq 0.05)\end{array}$ & p-value \\
\hline NW305 ++Fe vs NW305 -Fe & & & \\
$(\mathrm{N}=5658, \mathrm{k}=2231)$ & & & 0.42 \\
\hline Cytoplasm & 1988 & 188 & 0.99 \\
Endoplasmic reticulum & 363 & 78 & 0.95 \\
Golgi & 227 & 684 & 0.92 \\
Mitochondrion & 1794 & 68 & 0.05 \\
Peroxisome & 146 & 490 & $3 \mathrm{e}-3$ \\
Plasma membrane & 1140 & & 0.96 \\
\hline NW186 -Fe vs NW305-Fe & & 279 & 0.92 \\
(N=5641, k =852) & & 46 & 0.44 \\
\hline Cytoplasm & 1993 & 35 & 0.98 \\
Endoplasmic reticulum & 362 & 246 & 0.20 \\
Golgi & 224 & 26 & $4 \mathrm{e}-6$ \\
Mitochondrion & 1790 & 220 & \\
Peroxisome & 145 & & \\
Plasma membrane & 1127 & & \\
\hline
\end{tabular}




\subsubsection{Fe(III) citrate as iron source for A. niger and yeast-type fungi}

Finally, we investigated whether $A$. niger has the means to deal with citrate bound iron as iron source, and found that addition of Fe(III) citrate to the medium alleviates iron limitation and restores the growth phenotype (Table 3.5). In addition, the amount of iron added as Fe(III) citrate has the same effect on citrate production per glucose consumed as observed with $\mathrm{Fe}(\mathrm{II}) \mathrm{SO}_{4}$ (Figure 3.6).

Table 3.5: Final biomass [g/L] of A. niger NW305 and NW186 grown with Fe(III) citrate as iron source $\left(\mathrm{N}\right.$ source $\left.=\left(\mathrm{NH}_{4}\right)_{2} \mathrm{SO}_{4}\right)$.

\begin{tabular}{lccc}
\hline $\begin{array}{l}\text { Strain (major organic } \\
\text { acid(s) produced) }\end{array}$ & $-\mathrm{Fe}$ & $+\mathrm{Fe}$ & $++\mathrm{Fe}$ \\
\hline NW305 (oxalate, citrate) & $0.78 \pm 4 \mathrm{e}-3$ & $1.86 \pm 0.01$ & $3.28 \pm 0.01$ \\
NW186 (citrate) & $0.84 \pm 3 \mathrm{e}-3$ & $1.91 \pm 0.03$ & $3.09 \pm 0.01$ \\
\hline
\end{tabular}
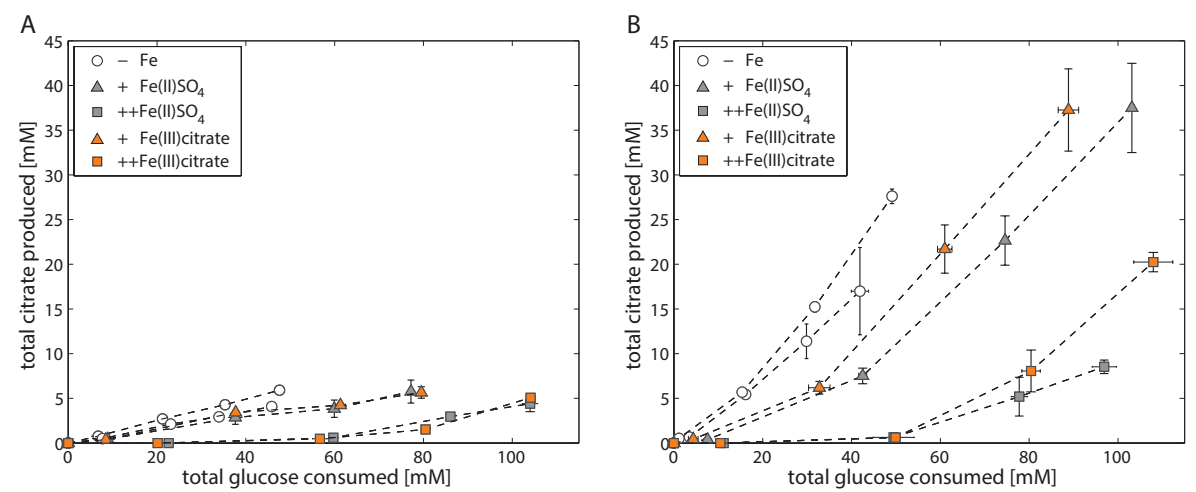

FIGURE 3.6. Iron-dependent citrate production of $\boldsymbol{A}$. niger NW305 and NW186 $\left(\mathbf{N}\right.$ source $\left.=\left(\mathbf{N H}_{4}\right)_{2} \mathbf{S O}_{4}\right)$. Total citrate production per glucose consumption of $A$. niger strains NW305 (A) and NW186 (B), grown without addition of iron (empty circles), or varying amounts of iron (triangles = iron limited, squares = iron excess), added either as $\mathrm{Fe}(\mathrm{II}) \mathrm{SO}_{4}$ (grey, filled symbols) or $\mathrm{Fe}$ (III) citrate (orange, filled symbols).

Most budding and fission yeasts, which do not produce own siderophores, are able to utilise iron complexed to xenosiderophores (Haas, 2014). To verify that $\mathrm{Fe}$ (III) citrate is a viable iron source for different yeast type fungi, we also tested the ability of Cyberlindnera jadinii, Cyberlindnera fabianii, Hanseniaspora uvarum, Kluyveromyces lactis, Saccharomyces cerevisiae, Wickerhamomyces 
anomalus and Wickerhamomyces ciferii to grow on Fe(III) citrate as iron source. With the exception of $C$. jadinii, which could not grow when Fe(III) citrate was added to the medium, all the strains were able to grow in all the conditions tested (either $\mathrm{Fe}(\mathrm{II}) \mathrm{SO} 4, \mathrm{Fe}(\mathrm{III})$ citrate or no iron added to the medium).

\subsection{Discussion}

\subsubsection{Citrate as overflow metabolite vs citrate as biological asset}

There are two possible interpretations for the results presented in Figure 3.2: i) A. niger citrate production is a result of metabolic overflow triggered by carbon excess relative to low iron availability, or ii) $A$. niger citrate production is a strategy to increase bioavailability of iron. The effect is essentially the same, only in hypothesis i), citrate is regarded as a "waste product" for the fungus, whereas in hypothesis ii), citrate is regarded as a biological asset enabling $A$. niger to cope with low iron stress.

To test which of these two hypotheses is more likely, we compared the responses of NW186 and NW305 to varying iron concentrations in the medium. Both of these strains are gluconate non-producers, and NW186 differs from its oxalate producing equivalent NW305 only by dysfunctional OahA (Han et al., 2007; Ruijter et al., 1999). This mutation deprives NW186 of the possibility to produce oxalate via the cytosolic route in which oxaloacetate is hydrolysed to oxalate and acetate (Figure 3.1); the established route of oxalate biosynthesis on glucose as carbon source (Kubicek et al., 1988). As a result, NW186 produces only citrate in major quantities, and decreasing the amount of iron added to the medium is directly reflected in increased citrate per glucose production in this mutant (Figure 3.2). In NW305, decreasing the amount of iron added to the medium increases both oxalate and citrate per glucose production (Figure 3.3).

According to hypothesis i), citrate would thus be regarded as an overflow metabolite alternative to oxalate, implying that carbon flow directed towards oxalate in NW305 stops short at citrate in NW186, and that increased extracellular oxalate secretion by NW305 -Fe is preceded by intracellular citrate accumulation. However, it has been shown that inhibition of $A$. niger aconitase does not affect oxalate production (Kubicek et al., 1988), nor does deletion of ATP-citrate lyase (Acl, Figure 3.1) (Meijer et al., 2009). In contrast, both of these modifications affect $A$. niger citrate production (Kubicek et al., 1988; Meijer et al., 2009). The contrasting effects of these modifications on citrate and oxalate production sug- 
gest that it is unlikely that a substantial amount of oxalate is derived from citrate as precursor, which is also in agreement with (Kubicek et al., 1988). Hypothesis i) is thus inconsistent with the observed A. niger phenotypes (Figures 3.2 and 3.3).

In the context of the discussion whether inhibition of TCA enzymes downstream of citrate is required for extracellular $A$. niger citrate accumulation, it is noteworthy to mention the aconitase iron-dependency (Kubicek and Röhr, 1977) (Kubicek and Röhr, 1978; La Nauze, 1966; Meixner-Monori et al., 1985; Szczodrak and Ilczuk, 1985). Aconitase catalyses the conversion of citrate to isocitrate via cis-aconitate. Aconitase inhibition due to lack of iron co-factor would explain the observed increase in citrate secretion upon iron limitation. In addition, it has been shown that aconitase, as well as other iron-dependent enzymes, is subject to HapX repression in A. nidulans (Hortschansky et al., 2007). However, A. niger aconitase, and other TCA cycle enzymes downstream of citrate, have been found to be active during citrate production, even when iron is not added to the medium (Karaffa and Kubicek, 2003; Kubicek and Röhr, 1985; La Nauze, 1966; Szczodrak and Ilczuk, 1985).

According to hypothesis ii) citrate is taking over the biological role of oxalate to increase bioavailability of iron. This hypothesis seems plausible, given that both oxalate and citrate have iron chelating properties (Gadd, 1999). We found that, similarly to iron siderophore biosynthesis pathways (Figure 3.5) leading to the A. niger iron siderophores coprogen B and ferrichrome (Franken et al., 2014), low iron stress is reflected in up-regulation of citrate and oxalate biosynthesis genes citA and oahA, respectively (Table3.3, Figure 3.7).

The role of CitA during citrate production has led to some controversy. CitA catalyses the condensation of acetyl-CoA with oxaloacetate to form citrate and coenzyme $\mathrm{A}$, and has been shown to have peak activity during $A$. niger citrate production (Kubicek and Röhr, 1977). However, up to 11-fold overproduction of CitA did not lead to the expected increase in A. niger citrate production (Ruijter et al., 2000). As a response to this work, this was later attributed to the activity of CitA within unmodified cells already being well above the activity which would account for the observed rate of $A$. niger citrate production (Ratledge, 2000; Ruijter, 2000). In another instance, the two mitochondrial citrate synthases in $A$. niger H915-1 were even found to be down-regulated during the citrate production phase (Yin et al., 2017).

In agreement with (Ratledge, 2000; Ruijter, 2000; Ruijter et al., 2000; Yin et al., 2017), we found that the difference in extracellular citrate accumulation was remarkably independent of citA expression; the gene was not differentially expressed between NW186 -Fe and NW305 -Fe (Table 3.3, Figure 3.7). Most 


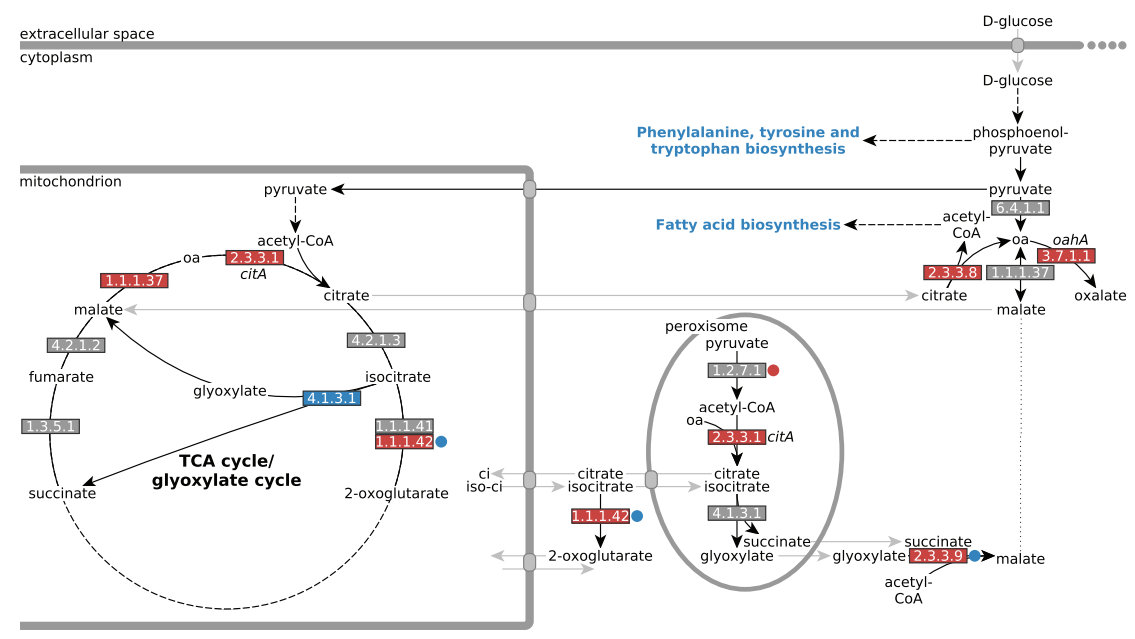

FIGURE 3.7. Differential expression of genes encoding enzymes involved in $A$. niger citrate and oxalate metabolism. Differential expression of genes directly involved in $A$. niger citrate and oxalate metabolism. EC numbers are coloured based on over- (red) or underexpression (blue) in response to iron limitation, i.e. in the control strain NW305 -Fe vs NW305 ++Fe. Small circles next to the EC numbers indicate that these reactions were differentially expressed in NW186 -Fe vs NW305 -Fe. Note that CitA (EC 2.3.3.1) has been found in mitochondria during citrate production, but has a peroxisomal as well as a mitochondrial targeting sequence, and could therefore be multilocated (Jaklitsch et al., 1991).

remarkably, though, citA was overexpressed in both NW186 -Fe and NW305-Fe compared to NW305 ++Fe, while citrate metabolising enzymes aconitase (EC 4.2.1.3) and NADP-IDH (EC 1.1.1.42) did not show iron-dependent transcriptional regulation (Table 3.3, Figure 3.7). Taken together, these observations suggest that citrate biosynthesis is actively up-regulated in response to iron limitation, but that there is another step of control determining whether citrate is ultimately metabolised (in the case of NW305-Fe), or secreted (in the case of NW186-Fe).

Organelle specific differential expression analysis shows the biggest differences in expression levels of plasma membrane proteins between all 3 conditions (Table 3.4). Therefore, it is likely that extracellular citrate accumulation is ultimately controlled at the transporter level. Transport of citrate was previously hypothesised to be the bottleneck of $A$. niger citrate production (Karaffa and Kubicek, 2003). In our case, we suggest that citrate is only secreted when there 
is a need to, i.e. in an attempt to increase iron availability under iron limited conditions, and when oxalate is not available for this purpose. Controlling the secretion of citrate irrespective of intracellular citrate biosynthesis, but rather based on the need to increase bioavailability of iron, offers another explanation why overexpression of citA does not, per se, lead to increased extracellular citrate accumulation (Ruijter et al., 2000). Note that the A. niger citrate exporter has, to date, not been identified, but that a list of citrate transporter candidates is provided by (Yin et al., 2017).

\subsubsection{Fe(III) citrate as iron source for A. niger and yeast-type fungi}

The Fe(III) citrate stability constant of 11.85 (expressed as log, Furia, 2006), although low in comparison to other microbial iron siderophores (Neilands, 1981), suggests that little metal is released from the complex, even at a low $\mathrm{pH}$. Thus, if $A$. niger lacks the means to deal with iron as metal ion complex with citrate, citrate secretion under low iron stress would effectively lead to an even more drastic iron shortage for the fungus. However, addition of $\mathrm{Fe}$ (III) citrate to A. niger culture medium restores the growth phenotype (Table 3.5, Figure 3.6), implying that, even if citrate is not employed as endogenous $A$. niger iron siderophore, the fungus has a means to deal with citrate bound iron as iron source.

Utilisation of $\mathrm{Fe}$ (III) citrate as exogenous iron siderophore complex has been shown to take place in various other microbes that do not naturally secrete citrate (Frost and Rosenberg, 1973), and of the yeasts we tested, only C. jadinii was unable to grow when Fe(III) citrate was added to the medium, although it grew well with $\mathrm{Fe}(\mathrm{II}) \mathrm{SO}_{4}$, and even when no iron was added to the medium. Active citrate uptake in the asexual state of $C$. jadinii (Candida utilis) has been shown to be subject to glucose repression (Cássio and Leao, 1991), and it could be that the utilisation of glucose in our experiments prevented $C$. jadinii from being able to utilise the Fe(III) citrate complex, whereas it is able to grow on other $\mathrm{Fe}(\mathrm{III})$ salts, such as $\mathrm{Fe}(\mathrm{III}) \mathrm{Cl}_{3}$ (Thomas and Dawson, 1978). This would imply that $C$. jadinii is not able to deal with Fe(III) citrate via RIA, which is in contrast to $S$. cerevisiae (Haas, 2014). A. niger utilisation of the Fe(III) citrate complex did not appear to be subject to glucose repression (Table 3.5, Figure 3.6), although measurable uptake of citrate in NW186 is only observed after glucose in the medium is depleted (Supplementary file 1). The difference between uptake systems when citrate is utilised as carbon source, or complexed citrate as iron source, appears thus to be a critical aspect that warrants further investigation, 
but is beyond the scope of this study.

From the data presented, we cannot distinguish whether A. niger employs RIA or imports the entire Fe(III) citrate complex. We found a number of putative "metalloreductases with ferric-chelate reductase activity" (Supplementary file 5), but these could be specific for citrate or any of the other A. niger iron siderophores. However, A. niger citrate uptake was studied by Netik et al., who showed that, while citrate export is increased under $\mathrm{Mn}^{2+}$ limited conditions, import of citrate only happens when $\mathrm{Mn}^{2+}$ is present in the medium (Netik et al., 1997). Uptake of citrate was inhibited by EDTA, and Netik et al. hypothesised that EDTA competes for the $\mathrm{Mn}^{2+}$ ions. They conclude that the citrate uptake system in A. niger either depends on $\mathrm{Mn}^{2+}$ symport, or, more likely, requires the metal ion chelated form of citrate as a substrate. The requirement of $\mathrm{Mn}^{2+}$ ions for citrate import could be partially replaced by $\mathrm{Mg}^{2+}, \mathrm{Fe}^{2+}$ or $\mathrm{Zn}^{2+}$ (but not $\mathrm{Cu}^{2+}$ ) ions (Netik et al., 1997), indicating that the $A$. niger citrate uptake system is not necessarily restricted to the citrate- $\mathrm{Mn}^{2+}$ complex, but could have a broader specificity for general citrate-metal ion complexes. It is therefore likely that iron is imported as Fe(III) citrate complex, although further experimental evidence would be required to establish citrate as definite $A$. niger iron siderophore, especially in the absence of a definitively identified uptake transporter, and bearing in mind that there are other A. niger iron siderophores, of which only two have been identified thus far (Franken et al., 2014). Furthermore, the ability of citrate to chelate other metal ions besides iron, and the fact that citrate import had a broad specificity for general citrate-metal-ion complexes (Netik et al., 1997), could imply that $A$. niger citrate secretion is a more general mechanism to facilitate the uptake of metal ions.

\subsubsection{Iron and the pH-dependency of $A$. niger organic acid production}

When not working with $A$. niger mutants tailored for citrate production, such as NW186, pH control is crucial to inhibit gluconate and oxalate production and enforce production of citrate instead (Ruijter et al., 1999). Certain aspects of the link between external $\mathrm{pH}$ and $A$. niger organic acid production have been elucidated on a molecular level. Glucose oxidase, the enzyme catalysing the first reaction in the conversion of $\mathrm{D}$-glucose to gluconate, has been shown to be stable only at pH 4-6 (Pazur and Kleppe, 1964), thus explaining the absence of gluconate at lower $\mathrm{pH}$ (Figure 3.1). The lack of oxalate at lower $\mathrm{pH}$ levels, on the other hand, can be explained by the finding that when the culture medium is below 
$\mathrm{pH} 2.5$, oxalate decarboxylase, the enzyme that degrades oxalate to $\mathrm{CO}_{2}$ and formate (Figure 3.1), is synthesised (Emiliani and Bekes, 1964). In a systems level approach, Andersen et al. (2009) formulated the hypothesis that the sequential production of organic acids by $A$. niger, and specifically oxalate and citrate, leads to the most efficient acidification of the medium based on the external $\mathrm{pH}$. As stated by the authors, continuous acidification of the environment provides a means to effectively outcompete other organisms that are not able to thrive at a low $\mathrm{pH}$, thereby providing an evolutionary advantage for the fungus.

In this study, we worked with $A$. niger mutants that are incapable of producing either gluconate or both gluconate and oxalate. Therefore, it was not necessary to exert control over external culture $\mathrm{pH}$ to enforce citrate production. In almost all the $A$. niger strains and conditions tested, the biggest $\mathrm{pH}$ drop (to $\sim \mathrm{pH} 2-4$, Supplementary file 6 ) was observed after 24 hours of growth. The $\mathrm{pH}$ continued to decrease steadily after that, albeit at a slower pace. This pattern is broken when iron is added to NW186 pre-grown without iron (Figure 3.2B, Supplementary file 1), or when glucose is depleted (Supplementary file 1). In both cases, the $\mathrm{pH}$ stops dropping and appears to even rise again (Supplementary Figures $2 \mathrm{~B}$ and $3 \mathrm{~B}$ in Supplementary file 1). This is likely due to consumption of citrate in the glucose depleted cultures, but it is not clear what the fungus is taking back up in the case of the iron pulse. Although it is tempting to link the rising $\mathrm{pH}$ to the net uptake of an iron-citrate complex, it is also possible that the $\mathrm{pH}$ rises due to an increased activity of $\mathrm{H}^{+}$symport of another iron-siderophore or compound.

A general observation in fungi is that organic acid secretion is actually higher at higher external $\mathrm{pH}$, and that there is a continuous influx and efflux of organic acids (Vrabl et al., 2012). Besides the discussed hypothesis that acidification of the medium might serve to outcompete other organisms, acidification of the environment is also a means of increasing iron solubility and thus bioavailability (Dutton and Evans, 1996; Gadd, 1999). Based on the results presented and discussed, we propose that citrate, and possibly also oxalate and gluconate, are not just secreted to acidify the medium, but that the sequential secretion of gluconate, oxalate, and then citrate is based on optimally increasing bioavailability of iron based on their own chelating properties at the given external $\mathrm{pH}$, and as such serve as $A$. niger iron siderophores. The fact that oxalate secretion precedes citrate secretion until $\mathrm{pH} \leq 2.5$ can be associated to the lower stability constant of the Fe(III) oxalate complex compared to the Fe(III) citrate complex (9.4 compared to 11.85 (Furia, 2006)), implying that the Fe(III) citrate complex is more stable at lower $\mathrm{pH}$ values.

The increased correlation between iron limitation and citrate production 
observed in the exclusively citrate producing $A$. niger mutant NW186, even at a $\mathrm{pH}$ that would usually be considered suboptimal for citrate production (Ruijter et al., 1999), is due to citrate being the only organic acid available for a task that would otherwise be shared between, and optimally adjusted to, multiple organic acids. The dependency of $A$. niger organic acid production on ambient $\mathrm{pH}$ also draws parallels to iron siderophore metabolism in Aspergillus nidulans, where, consistent with the insolubility of iron at alkaline $\mathrm{pH}$, production of $A$. nidulans iron siderophores increases with an increase in culture pH (Eisendle et al., 2004).

Based on these insights, our findings, and in line with the observation that $A$. niger imports citrate only as metal-ion complex (Netik et al., 1997), we suggests that increased citrate secretion under iron limited conditions is a physiological response to, rather than just a consequence of, low iron availability. Specifically, we propose that the reason $A$. niger citrate synthesis is actively up-regulated under iron limited conditions is because the fungus employs citrate as iron siderophore.

\subsection{Acknowledgements}

We want to thank Alex Kruis for interesting and fruitful discussions, Ruben van Heck for reading and commenting on the manuscript, and Wen Wu for help and feedback on the figures.

\section{Data availability}

RNA seq data:

EMBL-EBI (PRJEB20746), http://www.ebi.ac.uk/ena/data/view/PRJEB20746

\section{Supplementary information}

Supplementary files for this chapter can be found online at:

http://fungen.wur.nl/supplementary-information_odoni/

username: odoni

password: aniger 



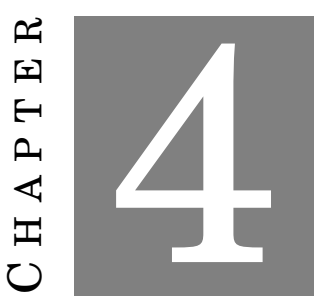

Aspergillus niger CITRATE EXPORTER REVEALED BY COMPARISON OF TWO ALTERNATIVE CITRATE PRODUCING CONDITIONS

\section{This chapter is adapted from:}

Odoni DI, Vazquez-Vilar M, van Gaal MP, Schonewille T, Tamayo-Ramos JA, Laothanachareon T, Suarez-Diez M, Schaap P. (2017) Aspergillus niger citrate exporter revealed by comparison of two alternative citrate producing conditions. In preparation. 


\subsection{Abstract}

Currently, there is no consensus regarding the mechanism underlying Aspergillus niger citrate biosynthesis and secretion, although it is amongst the most studied biotechnological production processes. Carbon excess relative to various other medium constituents is key, but the complex interplay between the limiting factors required for extracellular citrate accumulation remains elusive. Here, we show that the phenotype of increased extracellular citrate accumulation can have fundamentally different underlying mechanisms, depending on how this response was triggered. We found that varying the amount and supplement of an arginine auxotrophic $A$. niger strain induces increased citrate productivity. Transcriptomics analysis shows down-regulation of citrate metabolising enzymes in the conditions in which more citrate is accumulated extracellularly. This contrasts with the transcriptional adaptations triggered by iron limited conditions, which also induces citrate production. By combining data obtained from these two manners of inducing citrate production, we compiled a list of likely citrate transporter candidates. Two promising citrate exporter candidates were tested in the yeast Saccharomyces cerevisiae, of which one was successfully identified as citrate exporter. Our findings provide the first steps in untangling the complex interplay of different mechanisms underlying $A$. niger citrate accumulation, and we pinpoint, for the first time, a promising citrate exporter candidate, offering a valuable tool for improvement of $A$. niger as biotechnological cell-factory for citrate production. 


\subsection{Introduction}

Aspergillus niger citrate production is a notorious example of a production process that requires a unique combination of unusual nutrient and environmental conditions (Karaffa and Kubicek, 2003). Carbon excess relative to iron, zinc, copper, manganese, phosphorus, magnesium, potassium and nitrogen reportedly all leads to increased $A$. niger citrate production (Chesters and Rolinson, 1951; Kubicek and Röhr, 1977). The result of this is that, although A. niger citrate production has been subject to study since Currie's fundamental breakthroughs regarding $A$. niger citric acid fermentation 100 years ago (Currie, 1917), it is still not fully understood.

One problem might be that there are multiple factors at play, and studies therefore contradict each other depending on which aspect or time point of citric acid accumulation was investigated (Karaffa and Kubicek, 2003). Citric acid secretion has long been accepted to be a response to unfavourable intracellular conditions that lead to excess fluxes through the TCA cycle, and ultimately the undesired (from the perspective of the fungus) accumulation of citrate, i.e. overflow metabolism (Legiša and Mattey, 2007). Nevertheless, the viewpoint that citrate is solely an overflow metabolite is changing, and A. niger citrate secretion might also be regarded as a response to environmental conditions, such as competition (Andersen et al., 2009), or low iron bioavailability (Odoni et al., 2017b). While large scale production of citrate can probably be attributed to a combination of different mechanisms, increased understanding of all the various aspects that lead to increased citrate production can provide tools to further control and modulate citrate production.

Another important element of metabolic control can be found at transporter level (Karaffa and Kubicek, 2003), and overexpressing or introducing specific transporters for the product of interest can provide additional tools to overcome product limitation (van der Straat and de Graaff, 2014). Dynamic models of metabolism have highlighted the citrate exporter as one of the proteins whose overexpression could lead to increased citrate production rates (Alvarez-Vasquez et al., 2000). However, even though the citrate export system in A. niger has been described (Netik et al., 1997), no citrate exporter has yet been identified. Knowledge and characterisation of the $A$. niger citrate exporter would thus open new venues to further control and increase product formation.

Bioinformatics approaches have been developed to mine genome sequences for proteins of predefined functions. Amongst them, hidden Markov models (HMMs) represent multiple sequence alignments as position-dependent scoring systems, 
allowing variable conservation levels and alignment length (Eddy, 1998). In this way, HMMs are less sensitive to variations in the less conserved regions on the proteins. HMMs have been successfully used to identify glucose and xylose transporters in A. niger (Sloothaak et al., 2015, 2016b). However, construction of a HMM with reliable predictive power depends on the availability of biochemically characterised proteins with the function of interest. The scarcity of identified and biochemically characterised eukaryotic citrate exporters limits the predictive potential of an HMM in this case. Successful identification of citrate exporters would thus require combining in silico predictions with additional biological information.

Here, we explore how changes in media composition induce citrate production, and how combining multiple datasets can narrow down the list of putative citrate exporter candidates. For this, we worked with a defined $A$. niger $\arg B$ knock out mutant, which is unable to synthesise arginine. This mutation induces an "auxothropy" that can be overcome by media supplementation with either arginine or citrulline (Lenouvel et al., 2002). We studied the impact of supplement type and amount on citrate production, and performed a comparative transcriptome analysis to pinpoint the metabolic adaptations associated with the higher citrate producing condition. We compared the results of this study with previous data regarding citrate production triggered by low iron availability (Odoni et al., 2017b). Differences and similarities in the underlying transcriptomic landscape can help discern universal changes upon citrate production, or more specific changes given the condition, and serve as the starting point for the identification of putative citrate transporter candidates. We validated our approach by testing two promising citrate exporter candidates in Saccharomyces cerevisiae, and show that one of the transformants indeed accumulates a measurable amount of extracellular citrate. With this, we take the first steps in untangling the interplay between mechanisms regulating citric acid production in A. niger, and identify, for the first time, a promising $A$. niger citrate exporter candidate.

\subsection{Materials and methods}

\subsubsection{Strains, media and culture conditions}

We used $A$. niger strain NW186 (cspA1 $\triangle \arg B$ goxC17 prtF28), a $\triangle \arg B$ and pyrA6 derivative of NW185 (Ruijter et al., 1999), for this study. The transformation of NW186 with the A. niger pyrA gene, leaving only the $\triangle \arg B$ transformation marker intact, was described previously (Odoni et al., 2017b). 
To obtain spores, $A$. niger was grown (from glycerol stock), for 4 days, on complete medium (CM) agar plates, containing, per 1'000 mL: 2 g meat peptone, $1 \mathrm{~g}$ yeast extract, $1 \mathrm{~g}$ casamino acids, $0.3 \mathrm{~g}$ yeast ribonucleic acids, $15 \mathrm{~g}$ agar and minimal medium (MM) salts (MM salts, per 1'000 mL: $6 \mathrm{~g} \mathrm{NaNO}$, $1.5 \mathrm{~g}$ $\mathrm{KH}_{2} \mathrm{PO}_{4}, 0.5 \mathrm{~g} \mathrm{KCl}$ and $0.5 \mathrm{~g} \mathrm{MgSO}_{4} \cdot 7 \mathrm{H}_{2} \mathrm{O}$ ), added before sterilisation, and $1 \mathrm{~mL}$ vitamin solution (composition of vitamin solution, per $100 \mathrm{~mL}: 0.01 \mathrm{~g}$ thiamine, $0.10 \mathrm{~g}$ riboflavin-5P, $0.01 \mathrm{~g}$ p-aminobenzoic acid, $0.10 \mathrm{~g}$ nicotinamide, $0.05 \mathrm{~g}$ pyridoxine-HCl, $0.01 \mathrm{~g}$ pantothenic acid, $0.002 \mathrm{~g}$ biotin), $1 \mathrm{~mL}$ Vishniac (Vishniac and Santer 1957) solution, $50 \mathrm{mM}$ (9 g) glucose, and $0.02 \%$ arginine, added after sterilisation. After 4 days of growth, spores were harvested with $12-13 \mathrm{~mL}$ of Saline-Tween solution.

The fermentor experiments were carried out as transfer experiments. For the pre-growth, a total of $10^{6}$ spores/mL were inoculated in $1 \mathrm{~L}$ Erlenmeyer shake flasks containing $200 \mathrm{~mL}$ of $A$. niger production medium (PM, per 1'000 mL: $1.2 \mathrm{~g}$ $\mathrm{NaNO}_{3}$ or $\left.0.93 \mathrm{~g}\left(\mathrm{NH}_{4}\right)_{2} \mathrm{SO}_{4}, 0.5 \mathrm{~g} \mathrm{KH}_{2} \mathrm{PO}_{4}, 0.2 \mathrm{~g} \mathrm{MgSO}_{4} \cdot 7 \mathrm{H}_{2} \mathrm{O}\right), 50 \mathrm{~g} / \mathrm{L}(\sim 250$ $\mathrm{mM}$ ) glucose, $40 \mu \mathrm{L}$ Vishniac (Vishniac and Santer 1957) solution, and either $1.1 \mathrm{mM}\left(0.2 \mathrm{~g} \mathrm{~L}^{-1}\right)$ arginine or $5 \mathrm{mM}\left(0.88 \mathrm{~g} \mathrm{~L}^{-1}\right)$ citrulline supplement. After $24 \mathrm{~h}$ of pre-growth in Erlenmeyers, $11 \mathrm{~g}$ of $A$. niger mycelium was transferred to fermentors, containing the same PM medium as described. Note that, for a better understanding of the system, yeast extract was not used in any of the experiments.

The $S$. cerevisiae strain used for validation of the citrate transporter candidates was CENPK2-1D (MAT $\alpha$, his3D1, leu2-3_112, ura3-52, trp1-289, MAL2-8c, SUC2). Preparation of CENPK2-1D yeast electrocompetent cells and transformation was performed according to Suga and Hatakeyama (Suga and Hatakeyama, 2003). For yeast transformation, the cell suspension was mixed with plasmid or linear DNA, transferred to a pre-chilled cuvette (0.2 cm Gene Pulse, Bio-Rad), pulsed at $2.5 \mathrm{kV}, 25 \mu \mathrm{F}, 200 \Omega$ using Gene Pulser Xcell (Bio-Rad) and plated on synthetic medium agar-plates. Transformed cells were selected in synthetic media (YPD) with $2 \%$ (w/v) dextrose, $0.67 \%$ (w/v) Yeast Nitrogen Base without aminoacids (BD), $0.14 \%$ (w/v) Yeast Synthetic Drop-out Medium supplement without uracil, tryptophan, histidine and leucine (Sigma-Aldrich), $0.0076 \%$ (w/v) histidine, $0.0076 \%(\mathrm{w} / \mathrm{v})$ tryptophan and $0.038 \%(\mathrm{w} / \mathrm{v})$ leucine.

For the growth experiments, the yeast strains were first grown (from glycerol stock) for two days on YPD plates in an incubator at $30{ }^{\circ} \mathrm{C}$. One colony was picked and pre-grown in $10 \mathrm{~mL}(\exp 1)$ or $15 \mathrm{~mL}$ (exp2) liquid YPD medium described above, supplemented with $0.0076 \%$ uracil to allow growth of the parent strain. $100 \mu \mathrm{L}(\exp 1)$ or $300 \mu \mathrm{L}(\exp 2)$ of the pre-growth culture were inoculated in 100 
$\mathrm{mL}$ shake flasks containing $20 \mathrm{~mL}$ YPD medium described above, containing either $20 \mathrm{~g} / \mathrm{L}$ glucose (exp1 and exp2) or $20.44 \mathrm{~g} / \mathrm{L}$ glycerol (exp2). $\mathrm{CuSO}_{4}$ (final concentraion $1 \mathrm{mM}$ ) was added after $4 \mathrm{~h}$ of growth to all strains (even the parent strain), except where we indicated that the strains are non-induced.

\subsubsection{Metabolite analysis using HPLC}

Extracellular metabolite concentrations were determined by high-performance liquid chromatography (HPLC). An ICS5000 HPLC (Thermo Scientific), equipped with an Aminex HPX-87H column (BioRad) at $60{ }^{\circ} \mathrm{C}$, and coupled to a refractive index detector (Shodex RI-101, sample frequency $5 \mathrm{~Hz}$ ) and a Thermo UV/VIS detector $(\lambda=210 \mathrm{~nm}$ ), was used. Separations were performed by elution with $0.016 \mathrm{~N} \mathrm{H}_{2} \mathrm{SO}_{4}$ at a flow rate of $0.6 \mathrm{~mL} / \mathrm{min}$. An organic acid standard, containing oxalic acid, citric acid, malic acid, succinic acid and itaconic acid, and a separate glucose standard, with $2 \mathrm{mM}, 5 \mathrm{mM}, 10 \mathrm{mM}, 20 \mathrm{mM}$ (both organic acid standard and glucose standard), $100 \mathrm{mM}$ and $200 \mathrm{mM}$ (only glucose standard) were used to calculate calibration curves for quantification of the extracellular metabolite concentrations. Propionic acid (6 $\mathrm{mM})$ was used as an internal standard.

\subsubsection{RNA isolation and quality control}

RNA extraction from the A. niger mycelium were performed using the Maxwell ${ }^{\circledR}$ 16 LEV simplyRNA Tissue kit (Promega). Frozen mycelium ( 100 mg) of the sample was submerged in $400 \mu \mathrm{L}$ Homogenising buffer supplemented with $8 \mu \mathrm{L}$ 1-thioglycerol in a $2 \mathrm{~mL}$ Lysing matrix $\mathrm{C}$ tube (MP), prefilled with a mix of glass beads. Mycelium samples were disrupted using a FastPrep-24 instrument (MP). After disruption all liquid was transferred to a LEV RNA Cartridge. $200 \mu \mathrm{L}$ lysis buffer was added and the rest of the extraction was performed by a Maxwell MDx AS3000 machine (Promega) following the protocol. RNA integrity and quantity were assessed with an Experion system (Bio-Rad), and only high quality samples (RIN value $\geq 7$ ) were selected for whole transcriptome shotgun sequencing. Total RNA was sent directly to BaseClear (Leiden, The Netherlands).

\subsubsection{RNA sequencing and quality check, and RNA seq data processing}

RNA sequencing (RNA seq) and initial quality check was performed by BaseClear. The RNA seq data analysis was performed as described previously (Odoni et al., 2017b): The RNA seq reads were filtered using SortMeRNA v1.9 (Kopylova et al., 
2012) and Trimmomatic v0.32 (Bolger et al., 2014). Read mapping against $A$. niger ATCC 1015 (Andersen et al., 2011; Nordberg et al., 2013) was performed using STAR v2.5.0c (Dobin et al., 2013). Gene coverage calculations were performed using BEDTools v2.17.0 (Quinlan and Hall, 2010), and subsequently normalised for the respective library sizes. Differential expression analysis was performed using the $R$ package edgeR (Robinson et al., 2010). RNA seq normalisation and differential expression was performed simultaneously for each comparison. Genes with a count per million $(\mathrm{CPM}) \geq 1$ in at least two samples were considered to be expressed and kept for further analysis. Trimmed mean of M-values normalisation was performed as implemented in the $\mathrm{R}$ package edgeR. $\mathrm{P}$-values were corrected for multiple testing using the Benjamini-Hochberg procedure. The terms "differentially expressed" and "overexpressed" refer to differences in read counts per CDS, and denote a fold-change $\geq 1.5(\mathrm{FDR} \leq 0.05)$.

\subsubsection{Metabolic pathway enrichment analysis}

The protein products of the expressed genes were annotated using PRIAM (Claudel-Renard et al., 2003), and subsequently assigned to KEGG pathway maps (Kanehisa and Goto, 2000; Kanehisa et al., 2016). Metabolic pathway enrichment analysis was performed using the hypergeometric test implementation ("phyper") of the R software environment (Ihaka and Gentleman, 1996).

\subsubsection{Identification of possible citrate transporter candidates}

Proteins with transmembrane helix structures (from the A. niger ATCC 1015 in silico proteome) were identified using the stand-alone TMHMM 2.0 software package (Krogh et al., 2001). Protein localisation was predicted using the standalone protComp software (www.softberry.com). Hidden Markov models were built as described before (Sloothaak et al., 2015) from two sets of proteins ("citrate transport" and "GO:0015137") downloaded from the UniProt database (Consortium et al., 2014). The genomes used for the homology approach were downloaded from the JGI database (Nordberg et al., 2013): Aspergillus kawachii (Futagami et al., 2011), Aspergillus nidulans (Arnaud et al., 2011; Galagan et al., 2005), Aspergillus flavus (Arnaud et al., 2011), Aspergillus fumigatus (Nierman et al., 2005; Ronning et al., 2005), Aspergillus terreus (Arnaud et al., 2011), Yarrowia lipolytica (Dujon et al., 2004) and Saccharomyces cerevisiae (Dujon et al., 2004). 


\subsubsection{Yeast transformation}

The coding sequences of the two most promising citrate exporter candidates (ATCC 1015 protein IDs 1165828 and 212337) were PCR-amplified from A. niger N402 cDNA using the primers 1165828(1)_Fwd, 1165828(1)_Rev, 1165828(2)_Fwd, 1165828(2)_Rev and 212337_Fwd and 212337_Rev, respectively (Table 4.1). After amplification, both coding sequences were cloned into a derivative of the pYES-plasmid, whereby the GAL1 inducible promoter was replaced by the CUP1 inducible promoter (Kruis et al., 2017). The plasmid was PCR-amplified using the primers pYES-CUP1_Fwd and pYES-CUP1_Rev for 212337 cloning and pYES-CUP1(1)_BsaI_Fwd, pYES-CUP1(1)_BsaI_Rev, pYES-CUP1(2)_BsaI_Fwd, pYES-CUP1(2)_BsaI_Rev for 1165828 (Table 4.1). All PCR-amplifications were performed with the Q5 polymerase following the manufacturer's protocol. The coding sequence of the citrate exporter candidate 1165828 was cloned using Golden Gate. The Golden Gate reaction mixture was prepared as follows: $400 \mathrm{U}$ of T4 DNA ligase (NEB), $10 \mathrm{U}$ of BsmBI (NEB), $1.5 \mu \mathrm{L}$ of BSA ( $1 \mathrm{mg} / \mathrm{mL}), 1.5$ $\mu \mathrm{L}$ of T4 DNA ligase buffer (NEB), $40 \mathrm{fmol}$ of each PCR product, and water to bring the volume up to $15 \mu \mathrm{L}$. The reaction mixture was incubated in a thermocycler according to the following program: $37^{\circ} \mathrm{C}$ for 10 min prior to 25 cycles of digestion-ligation $\left(37^{\circ} \mathrm{C}\right.$ for $3 \mathrm{~min}, 16^{\circ} \mathrm{C}$ for $4 \mathrm{~min}$ ) followed by a final digestion step (55 ${ }^{\circ} \mathrm{C}$ for $10 \mathrm{~min}$ ) and a heat inactivation step $\left(80^{\circ} \mathrm{C}\right.$ for $\left.10 \mathrm{~min}\right)$. E. coli $\mathrm{DH} 5 \alpha$ competent cells were transformed with $1 \mu \mathrm{L}$ of the digestion-ligation reaction, and transformants were selected on ampicillin plates. Plasmid extraction was performed using the GeneJET Plasmid Miniprep kit (Thermo Fisher Scientific) following the manufacturer's instructions. CENPK2-1D competent cells were then transformed with a sequence-verified plasmid. The coding sequence of the citrate exporter candidate 212337 was cloned using the yeast homologous recombination assembly strategy. For the in-yeast assembly, CENPK2-1D cells were directly transformed with $100 \mathrm{ng}$ of the PCR-amplified pYES-CUP1 plasmid and an equimolar amount of insert, and plated on selective medium. Positive transformants were picked and grown overnight in liquid medium. Plasmids were extracted using the GeneJET Plasmid Miniprep kit (Thermo Fisher Scientific) following the manufacturer's instructions with minor modifications. Lysis was performed by transferring the cells to Lysing MatrixC $2 \mathrm{~mL}$ tubes (MP Biomedicals) and homogenising them for 40 seconds with a FastPrep-24 from MP Biomedicals. $E$. coli DH5 $\alpha$ competent cells were transformed into with the extracted plasmids for propagation, and then again extracted using the GeneJET Plasmid Miniprep kit (Thermo Fisher Scientific) following the manufacturer's 
instructions and sequenced for insert verification.

Table 4.1: Primers used for the assembly of the citrate exporter candidates expression plasmids.

\begin{tabular}{|c|c|}
\hline 212337_Fwd & acaaactgatcgatgccaccatggetgtgaattttccatgg \\
\hline 212337_Rev & ctaattacatgatgcggeccttagacgccaacgtgggtatg \\
\hline pYES-CUP1_Fwd & gggccgcatcatgtaattag \\
\hline pYES-CUP1_Rev & ggtggcatcgatcagtttg \\
\hline 1165828(1)_Fwd & catacgtctcaatgtcttcaaccacgtcttc \\
\hline 1165828(1)_Rev & tagcgtctctatacgatgcaaaccatttgc \\
\hline 1165828(2)_Fwd & ctacgtctcagtatccetcgetgccatatt \\
\hline 1165828(2)_Rev & tagcgtctcacctagttgccgttggctttg \\
\hline pYES-CUP1(1)_BsaI_Fwd & ctacgtctcataggggccgcatcatgtaattag \\
\hline pYES-CUP1(1)_BsaI_Rev & tagcgtctctgtgacgcattgggtcaacag \\
\hline pYES-CUP1(2)_BsaI_Fwd & catcgtctcatcaccettgtcatctaaacc \\
\hline pYES-CUP1(2)_BsaI_Rev & tagcgtctctacatggtggcatcgatcagtttg \\
\hline
\end{tabular}

\subsection{Results and discussion}

\subsubsection{A. niger citrate and biomass production}

To study the mechanisms underlying extracellular citrate accumulation, and use these insights to pinpoint putative citrate exporter candidates, we compared data from two different experimental setups (Figure 4.1a). In the present study, we worked only with the A. niger N402 derivative NW186 (Ruijter et al., 1999). The present experiment was compared to data from the experiment described in Odoni et al. (2017b), in which we used both NW186 and the control strain NW305 (Ruijter et al., 2003). Both NW186 and NW305 have a mutation in glucose oxidase (GoxC, goxC17), making these strains gluconate non-producers. In addition, NW186 has a mutation in oxaloacetate acetylhydrolase (OahA, prtF28), making this strain an oxalate non-producer (Ruijter et al., 1999). As a result, NW305 is able to produce both oxalate and citrate, whereas NW186 only produces major amounts of citrate. Both of these strains are $\arg B$ knock out mutants, making them arginine auxotrophs (Lenouvel et al., 2002). Supplementation of the medium with either arginine or citrulline can restore growth of the $\triangle a r g B$ mutants, but we found that addition of excess $(5 \mathrm{mM})$ citrulline (NW186 +Fe_c) increases total citrate production when compared to addition of (1.1 mM; "standard" condition) arginine (NW186 +Fe_a). Thus, as can be seen Figure 4.1b, we can influence $A$. niger citrate production by either limiting iron availability 
(iron experiment), or substituting the arginine supplement with excess citrulline (supplement experiment).

Citrate production yields of the considered conditions are shown in Table 4.2. Total citrate yield is almost doubled in NW186 +Fe_c compared to NW186 +Fe_a (Table 4.2). As expected, addition of excess citrulline does not only increase the citrate productivity (g/L/h, Figure $4.1 \mathrm{~b}$ ), but also has an overall stimulating effect on metabolism; it leads to increased glucose consumption (Figure 4.1b), doubled final biomass (Table 4.2), and increased $\mathrm{CO}_{2}$ production $(337.79 \pm 15.86 \mathrm{mM}$ in $\mathrm{NW} 186+\mathrm{Fe} \_\mathrm{c}$, and $265.51 \pm 9.28 \mathrm{mM}$ in NW186 +Fe_a). On the other hand, not adding iron to the culture medium increases the citrate per glucose production rate (Figure 4.1b), but total biomass production remains limited (Table 4.2), and reduced glucose consumption is observed (Figure 4.1b). These differences between both experiments imply that there are two quite different mechanisms at play, although both lead to an increase in extracellular citrate accumulation.

Table 4.2: Final biomass and citrate yields of $A$. niger NW305 and NW186 grown with varying iron concentrations or supplements in the medium. Suppl. = supplements; $\mathrm{a}=$ arginine, $\mathrm{c}=$ citrulline. ${ }^{1}$ Data from Odoni et al. $(2017 \mathrm{~b})$.

\begin{tabular}{llcclrl}
\hline \multirow{2}{*}{ Exp. setup } & \multirow{2}{*}{ Strain } & \multirow{2}{*}{ Fe } & \multirow{2}{*}{ Suppl. } & \multirow{2}{*}{ Final biomass [g] } & \multicolumn{2}{c}{ Citrate yield } \\
& & & & & \\
& & & & \\
[g biomass] & [g/g substrate] \\
\hline shake flask & NW305 $^{1}$ & ++ & a & $0.35 \pm 0.02$ & $0.67 \pm 0.07$ & $0.02 \pm 0.002$ \\
shake flask & NW305 $^{1}$ & - & a & $0.19 \pm 0.02$ & $4.92 \pm 0.35$ & $0.11 \pm 0.002$ \\
shake flask & NW186 $^{1}$ & - & a & $0.22 \pm 1 \mathrm{e}-03$ & $21.88 \pm 1.51$ & $0.46 \pm 0.02$ \\
fermentor & NW186 $^{*}+$ & a & $1.87 \pm 0.04$ & $1.80 \pm 0.14$ & $0.17 \pm 0.03$ \\
fermentor & NW186 & + & c & $3.96 \pm 0.09$ & $2.98 \pm 0.35$ & $0.31 \pm 0.03$ \\
\hline
\end{tabular}

\subsubsection{RNA seq analysis}

Transcriptomic data corresponding to the iron experiment were presented in Odoni et al. (2017b). Here, we performed transcriptomics analysis on the supplement experiment, i.e. NW186 grown with $1 \mathrm{~g} / \mathrm{L} \mathrm{Fe}(\mathrm{II}) \mathrm{SO}_{4}$ added to the medium, and supplemented with either $1.1 \mathrm{mM}$ arginine or $5 \mathrm{mM}$ citrulline. RNA for RNA sequencing (RNA seq) was extracted after $48 \mathrm{~h}$ of growth. The annotated genome of A. niger ATCC 1015 (Andersen et al., 2011) was used as reference to map the RNA seq reads. Genes with count per million $(\mathrm{CPM}) \geq 1$ were considered to be expressed (Supplementary file 1). The supplement change from arginine to excess citrulline induces major transcriptional adaptations, and over $20 \%$ of the annotated genes are differentially expressed (Table 4.3, Supplementary file 2). 

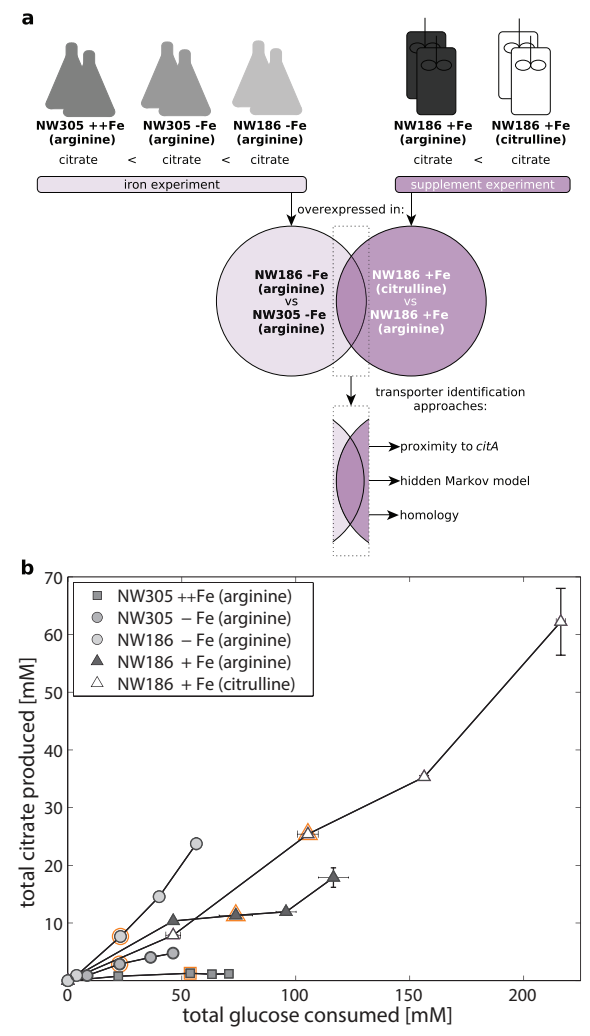

FIGURE 4.1. a) Experimental setup and citrate exporter identification workflow. In the iron experiment, performed in shake flasks, extracellular citrate accumulation was modulated by reducing iron availability in the culture medium (Odoni et al., 2017b). In the supplement experiment, performed in fermentors, extracellular citrate accumulation was modulated by substituting the arginine supplement with excess citrulline. Lists of differentially expressed genes in both experimental setups were compared to obtain a shortlist of the most promising citrate exporter candidates. The list of citrate exporter candidates was further narrowed down following the indicated bioinformatic approaches. b) Total citrate production per glucose consumption of NW186 and NW305. The A. niger strains were grown in shake flasks (iron experiment) or fermentors (supplement experiment), without addition of iron (circles), or varying amounts of $\mathrm{Fe}(\mathrm{II}) \mathrm{SO}_{4}$ (triangles $=1 \mathrm{~g} / \mathrm{L}$, squares $=10 \mathrm{~g} / \mathrm{L}$ ), and supplemented with either arginine (filled symbols) or excess citrulline (empty symbols). Sample points for RNA extraction $(\mathrm{t}=48 \mathrm{~h})$ are marked in orange. Measurement points were taken once every $24 \mathrm{~h}$ and show the average of two biological replicates. 
Table 4.3: RNA seq mapping and differential expression analysis

\begin{tabular}{|c|c|c|}
\hline $\begin{array}{l}\text { Strain } \\
\text { culture condition }\end{array}$ & $\begin{array}{l}\mathrm{NW} 186+\mathrm{Fe} \\
\text { arginine }\end{array}$ & $\begin{array}{l}\mathrm{NW} 186+\mathrm{Fe} \\
\text { citrulline }\end{array}$ \\
\hline \# reads after QC filtering & $42 ’ 029 ' 453$ (1) & $30 ’ 895 ' 435$ (1) \\
\hline & 49'141’964 (2) & $68 ’ 512 ' 663(2)$ \\
\hline Uniquely mapped reads & $85.16 \%(1)$ & $57.87 \%(1)$ \\
\hline (against ATCC1015 CDS) & $68.80 \%(2)$ & $68.27 \%(2)$ \\
\hline \# genes expressed $(\mathrm{CPM} \geq 1)$ & 9620 & 9705 \\
\hline $\begin{array}{l}\text { \# genes differentially expressed, } \\
\log 2 \mathrm{FC} \text { threshold } \geq 0.58(\mathrm{FDR} \leq 0.05)\end{array}$ & \multicolumn{2}{|c|}{2385} \\
\hline $\begin{array}{l}\text { \# EC covered (mapped to KEGG path- } \\
\text { ways) }\end{array}$ & \multicolumn{2}{|c|}{466} \\
\hline $\begin{array}{l}\text { \# EC differentially expressed, } \\
\log 2 \mathrm{FC} \text { threshold } \geq 0.58(\mathrm{FDR} \leq 0.05)\end{array}$ & \multicolumn{2}{|c|}{173} \\
\hline
\end{tabular}

From the 466 enzyme commission (EC) numbers that have been included in KEGG maps of metabolism (Kanehisa and Goto, 2000; Kanehisa et al., 2016), and can be found in the annotated genome of $A$. niger ATCC 1015, $37 \%$ show differential expression. Pathway enrichment analysis shows prevalence of differentially expressed genes in pathways associated to biomass formation, such as starch and sucrose metabolism, and pathways related to amino acid biosynthesis, such as phenylalanine, tyrosine and tryptophan biosynthesis and metabolism, and valine, leucine and isoleucine biosynthesis (Supplementary file 3). There is also an enrichment of differentially expressed genes related to fatty acid biosynthesis, and synthesis and degradation of ketone bodies pathways, upon addition of excess citrulline instead of arginine to the medium (Supplementary file 3). Note that, although we observed higher glucose consumption in NW186 +Fe_c, glycolysis as a pathway did not show enrichment of differentially expressed genes.

Most of the pathways enriched in the supplement experiment (Supplementary file 3) were also enriched in the iron experiment (Odoni et al., 2017b). Phenylalanine, tyrosine and tryptophan biosynthesis, and fatty acid biosynthesis pathways showed similar behaviours in both experiments, and most of the enzymes in these pathways are down-regulated in the condition with higher citrate production.

Looking at enzymes that we connected previously to organic acid production in filamentous fungi (Odoni et al., 2017a), we found that alternative oxidase (AOX, or non-electrogenic ubiquinol oxidase, EC 1.10.3.11) was up-regulated in the condition corresponding to the highest citrate secretion in both the experimental setups (Figure 4.2, Supplementary file 2). In the supplement experiment, 
up-regulation of AOX was accompanied with down-regulation of cytochrome c reductase, whereas in the iron experiment, this was not the case (Figure 4.2, Supplementary file 2). This might be due to high intracellular accumulation of citrate preceding its secretion in NW186 -Fe_a; it was found that incubating detached roots of the plant Poa annoa with citrate increased protein concentration of AOX without actually increasing the activity of the alternative respiration pathway itself (Millenaar et al., 2002). The authors of the study hypothesised that the chelating properties of citrate might lead to the withdrawal of the $\mathrm{Fe}$ in the active centre of AOX, thereby rendering the protein inactive and evoking increased transcription of AOX to compensate for the inactive protein (Millenaar et al., 2002). Interestingly, iron limitation in itself is not enough to evoke this response, but the presence of an iron chelator seems to be necessary, e.g. citrate or o-phenanthroline (as shown for the yeast Hansenula anomala (Minagawa et al., 1990)).

As we have previously also hypothesised that citrate, as a natural ironchelator, is employed as A. niger iron siderophore (Odoni et al., 2017b), we analysed expression levels of genes involved in iron siderophore biosynthesis and transport (Supplementary file 4). Most of the genes are overexpressed in NW186 +Fe_c vs NW186 +Fe_a. This is likely a result of the fast growing NW186 $+\mathrm{Fe} \_c$ cultures depleting the iron in the medium, which is present only in trace amounts and from whatever leaks from the metal parts of the fermenters.

Interestingly, the enzymes converting citrulline to arginine showed no differential expression in the supplement experiment (Figure 4.2, Supplementary file 3). Citrulline, when added in excess, will not only supplement the $\triangle a r g B$ mutation, but also act as nitrogen source, thereby alleviating nitrogen limitation. Highly expressed enzymes connected to nitrogen limitation and amino acid catabolism (discussed in (Odoni et al., 2017a)) were down-regulated in NW186 +Fe_c compared to NW186 +Fe_a (Figure 4.2). In our experimental setup, increased citrate production was thus not due to nitrogen limitation in either of the two experiments; In NW305 -Fe_a and NW186-Fe_a, iron was the limiting factor (Odoni et al., 2017b), whereas addition of excess citrulline in NW186 +Fe_c provides an extra source of nitrogen. However, in contrast to the general consensus, the accompanying increased biomass formation did not come at the expense of citrate yield (Table 4.2). The question remains why addition of excess citrulline had such a strong effect on $A$. niger citrate productivity (Figure 4.1b).

Analysis of the expression of enzymes involved in citrate metabolism revealed quite a contrasting transcriptomic landscape in the two experimental setups (Figure 4.3). While citrate biosynthesis genes are up-regulated in the condition 


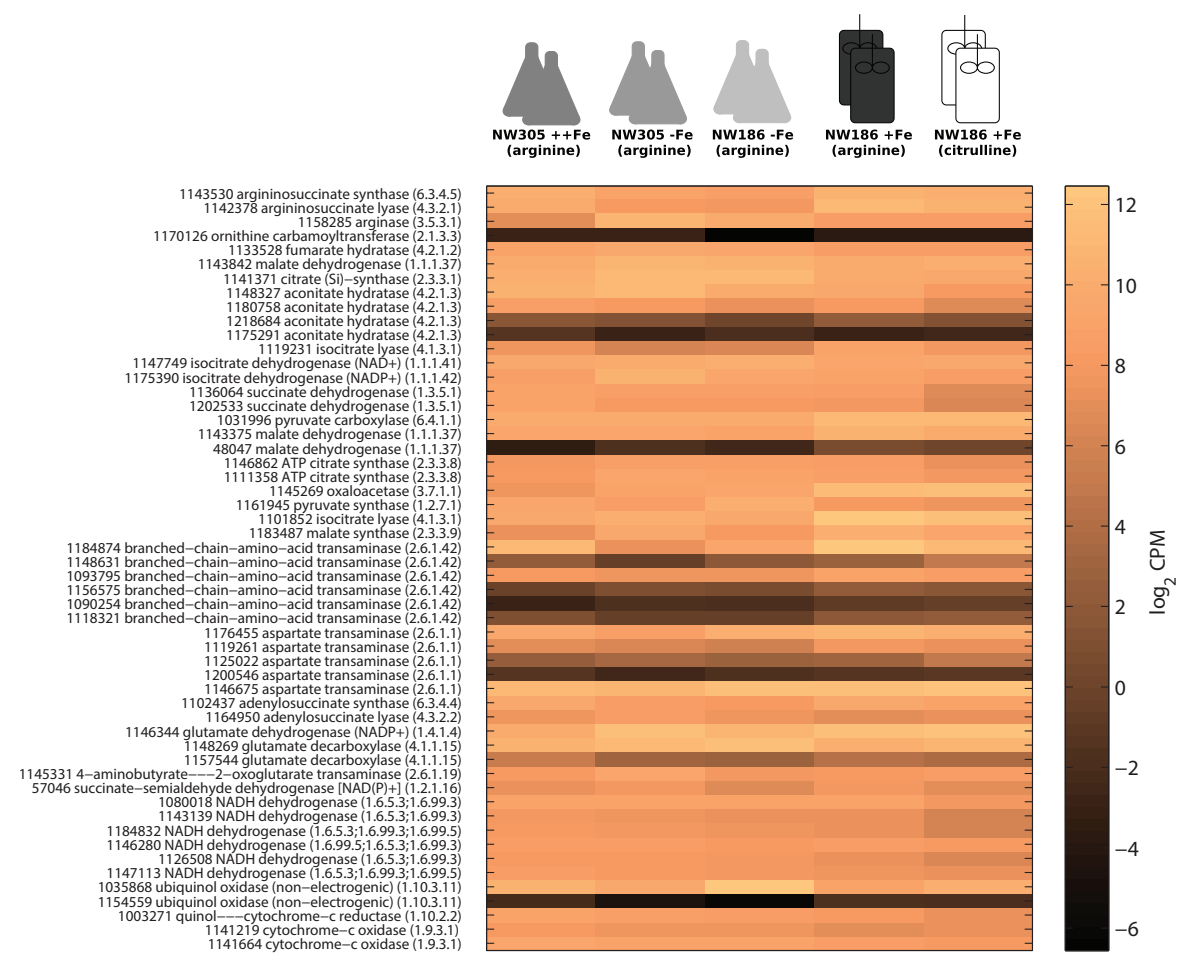

Figure 4.2. Gene expression levels ( $\left.\log _{2} \mathrm{CPM}\right)$ of enzymes involved in citrate metabolism, either by direct association or as found previously in literature. ATCC 1015 protein identifiers are given next to the enzyme names. Included are enzymes comprising the ornithine and TCA cycles, enzymes involved in amino acid turnover, and components of respiration.

corresponding to increased extracellular citrate secretion in the iron experiment, there is down-regulation of citrate metabolising enzymes in the condition corresponding to increased extracellular citrate accumulation in the supplement experiment. 


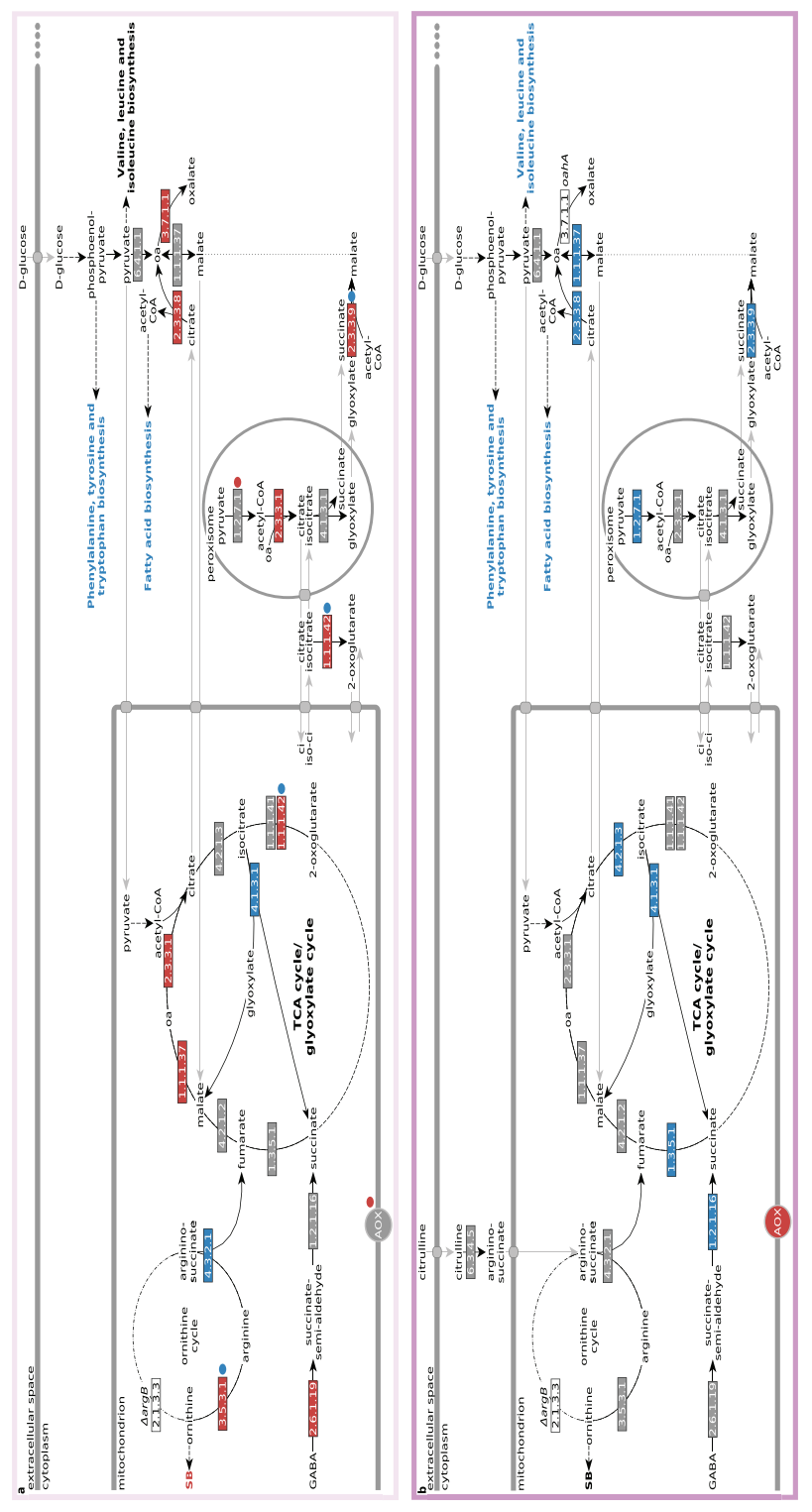

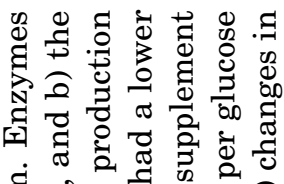

घं

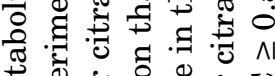

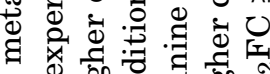

a d.

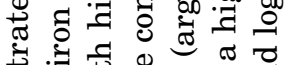

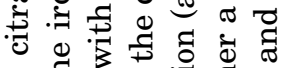

ఏ ‡.

న స

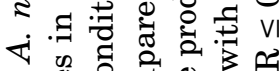

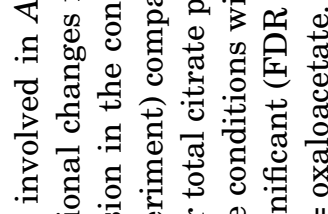

की

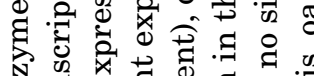

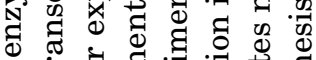

$\infty \begin{gathered}0 \\ 0\end{gathered}$

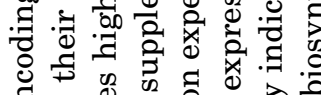

过

\& 00 . ఫ

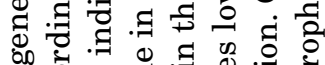

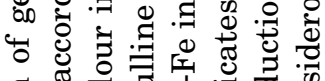

융

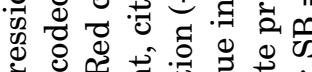

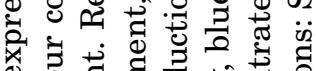

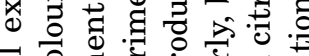

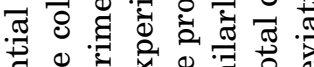

प्च.

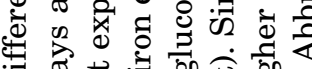

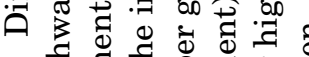

क

䚺要

氜 
The contrasting transcriptomic landscapes (Figure 4.3, simplified in Figure 4.4) further support the initial hypothesis that there are two distinct mechanisms underlying the phenotype of increased citrate accumulation in the two experimental setups. Triggering increased citrate secretion by limiting Fe availability in the medium results in the highest citrate yields per biomass produced and substrate consumed (Figure 4.1b, Table 4.2). This behaviour, together with the observation that the citrate biosynthesis gene citA shows active up-regulation under Fe-limited conditions, but not per se under conditions in which citrate secretion is high (Figure 4.3, but also (Ratledge, 2000; Ruijter, 2000; Ruijter et al., 2000)), has led to our hypothesis that citrate might serve as $A$. niger iron siderophore (Odoni et al., 2017b). However, although efficient in terms of yield per biomass produced and substrate consumed, absolute citrate productivity $(\mathrm{g} / \mathrm{L} / \mathrm{h})$ remains low (Figure 4.1b, Table 4.2).

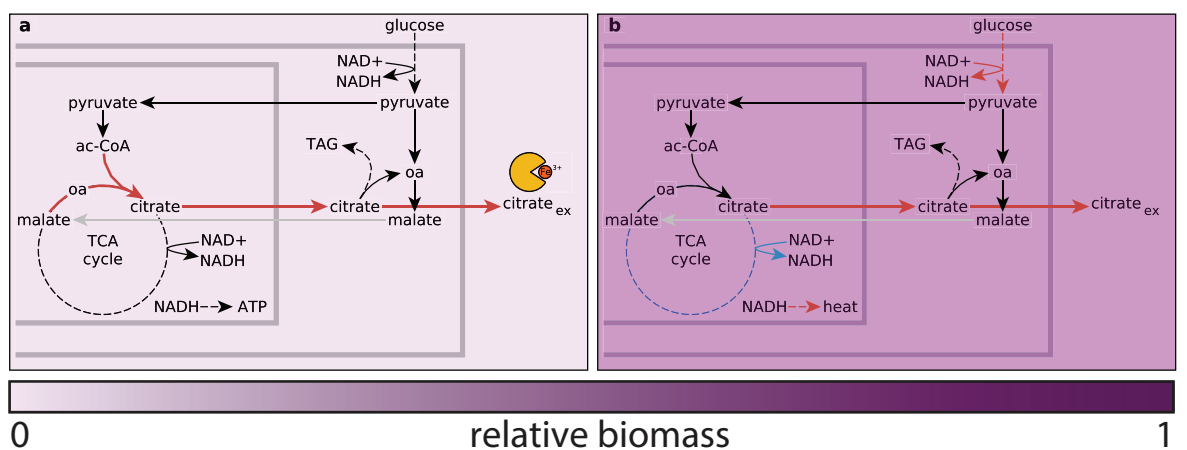

FigURE 4.4. Simplification of two proposed mechanisms underlying $A$. niger citrate production, with red = high flux, and blue = low flux. a) Citrate as biological asset: Under iron limitation, citrate biosynthesis appears to be actively up-regulated, consistent with a possible role for citrate as $A$. niger iron siderophore (Odoni et al., 2017b). b) Citrate as overflow metabolite: The fast glucose consumption in the condition where $A$. niger is supplemented with excess citrulline leads to high glycolytic flux, which in turn results in excess production of NADH. Removal of citrate from the TCA cycle prevents further production of NADH downstream of citrate. In addition, $\mathrm{NADH}$ re-oxidation has to be de-coupled from ATP production via the alternative oxidase respiration pathway.

In the supplement experiment, we observed a higher glucose consumption rate when NW186 was supplemented with excess citrulline (Figure 4.1b), although this was not reflected in up-regulation of glycolysis at transcript level 
(Supplementary file 3). A high glucose consumption rate results in high glycolytic flux that will lead to excess $\mathrm{NADH}$, the turn-over of which might be limited by the capacity of further biosynthetic processes (Karaffa and Kubicek, 2003). The down-regulation of cytochrome-dependent respiratory enzymes, and the up-regulation of alternative oxidase (AOX, or non-electrogenic ubiquinol oxidase) when supplementing NW186 with excess citrulline, indicate a switch from cytochrome-dependent respiration to alternative respiration mediated by AOX (Karaffa and Kubicek, 2003). This effectively decouples ATP generation and $\mathrm{NADH}$ re-oxidation, as the reduction of molecular oxygen to water by AOX bypasses proton translocation via the oxidative phosphorylation complexes III and IV, resulting in a lower ATP yield (Joseph-Horne et al., 2001). Down-regulation of TCA cycle enzymes downstream of citrate in NW186 +Fe_c (Figure 2B), prevents citrate to be further metabolised, which might be a mechanism to prevent further generation of NADH (Gallmetzer and Burgstaller, 2002).

\subsubsection{Transporter identification and validation}

To assemble a list of promising citrate transporter candidates, we followed three complementary approaches. First, we looked at the problem in the context of citrate as iron siderophore. Fungal iron homeostasis genes have been found to be clustered in the genome (Andersen et al., 2011), and it is not uncommon that fungal transporters are encoded by genes in close proximity to the genes encoding biosynthesis of their transport target (van der Straat et al., 2014). Thus, our first selection of citrate transporter candidates are the genes located around citA in the ATCC 1015 genome, and which encode proteins with at least one predicted transmembrane helix (tmh) domain (Supplementary file 5, Sheet "cluster_approach").

Next, we constructed two profile hidden Markov models (HMMs) from multiple sequence alignments of biochemically characterised citrate transporters obtained from the UniProt database (Consortium et al., 2014). The HMMs were used to identify and score new citrate transporter candidates in the ATCC 1015 in silico proteome (Supplementary file 5, Sheet "HMM_approach"). The highest scoring candidate, with protein ID 1141368, also fits with the previous approach, as it is located very close to citA (see Supplementary file 5, Sheet "cluster_approach"). Another high scoring protein, with protein ID 1155853, is also in the genomic vicinity of $c i t A$, although it had a much lower HMM score. Note that most of the citrate transporter candidates identified with this approach are predicted to be located in the mitochondria or other cell-organelles rather than the plasma- 
membrane (pm). This is likely due to a bias in the sequences used to build the HMMs. For one of the two HMMs ("citrate transporter"), we obtained 393 proteins from the UniProt database, of which only 98 are predicted to be located in the pm. However, 12 of these are of bacterial origin, further biasing the predictions towards transporters of cell-organelles rather than the eukaryotic pm. A similar pattern is obtained with the HMM constructed from proteins with specific citrate transporter activity ("GO:0015137"): here, we obtained 74 proteins, of which 43 are predicted to be located in the pm. Again, many of these (16) are of bacterial origin. Thus, the results from the HMM approach can be used to condense a list of likely citrate transporters in various cell-organelles, but is not suitable to pinpoint $A$. niger citrate exporter candidates.

Last, we identified all plasmalemmal proteins and selected them attending to the presence and similarity of homologues in other Aspergilli or yeast that are known to either produce (A. kawachii, Yarrowia lipolytica) or not produce (A. flavus, A. terreus, S. cerevisiae) citrate (Supplementary file 5, Sheet "homology_approach"). We combined these three approaches with the expression levels and predicted localisation of the identified proteins, and ranked these transporters based on the expression data obtained from our two experimental setups.

Based on our pre-selection (Supplementary file 5), we chose the two proteins (protein IDs 1165828 ("CitT_12tmh") and 212337 ("CitT_4tmh")) as two likely citrate exporter candidates for further validation in S. cerevisiae. CitT_12tmh has 12 transmembrane helices (tmhs), and is the top-ranking citrate exporter candidate based on its expression levels in the two experimental setups, and its higher similarity to transporters in A. kawachii and Y. lipolytica compared to the non-citrate producing fungi (Supplementary file 5, Sheet "homology_approach"). CitT_4tmh has 4 tmhs, and was chosen based on being the only transporter candidate in the close vicinity of citA that is predicted to be located in the pm, although its low expression levels suggest lesser importance as actual citrate exporter (Supplementary file 5, Sheet "cluster_approach").

$S$. cerevisiae was transformed with plasmids containing either one of the putative citrate exporter candidates under the control of a copper inducible promoter (CUP1). In the first growth experiment, we induced expression of the transporter proteins after $4 \mathrm{~h}$ with $1 \mathrm{mM} \mathrm{CuSO}_{4}$, and found that neither the untransformed control strain, nor the strain containing CitT_4tmh, secreted any citrate. However, the strain containing CitT_12tmh accumulated a small amount of extracellular citrate, indicating that this could be an $A$. niger citrate exporter. To further verify our initial results, we grew $S$. cerevisiae containing 
CitT_12tmh with either glucose or glycerol in the medium, and either did or did not induce expression with $\mathrm{CuSO}_{4}$. Reconfirming our initial observations, we found measurable accumulation of citrate in the extracellular medium only in $S$. cerevisiae in which CitT_12tmh was induced, but not in the non-induced transformant, nor in the untransformed parent strain (Figure 4.5).

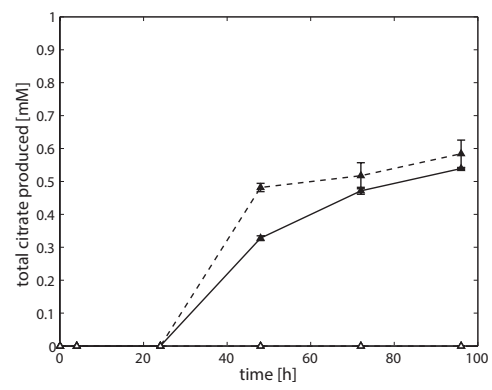

FIGURE 4.5. Citrate secretion in $S$. cerevisiae + CitT_12tmh, with solid line = grown on glucose, dashed line = grown on glycerol, filled symbols $=$ induced, empty symbols $=$ non-induced. Note that there was no measurable extracellular citrate accumulation in the untransformed parent strain. Measurements indicate the average of two biological replicates.

\subsection{Conclusion}

In the present work, we compared the trancriptomic landscape differences in two experimental setups in which increased $A$. niger citrate secretion was triggered by either limiting iron availability, or supplementing the fungus with excess citrulline instead of arginine. Our analyses of the underlying transcriptomic landscape shows that extracellular citrate accumulation can be the answer to multiple environmental conditions. As such, we propose that there is no such thing as the mechanism underlying $A$. niger citrate production and secretion. Finally, in combining the expression data of both the iron and supplement experiments with three different in silico approaches, we condensed the A. niger transportome into a shortlist of the most promising citrate transporter candidates, leading to the identification of an A. niger citrate transporter that shows citrate export in transformed $S$. cerevisiae. With this, we provide a framework for further improvement of $A$. niger as biotechnological production host. 


\section{Supplementary information}

Supplementary files for this chapter can be found online at:

http://fungen.wur.nl/supplementary-information_odoni/

username: odoni

password: aniger 


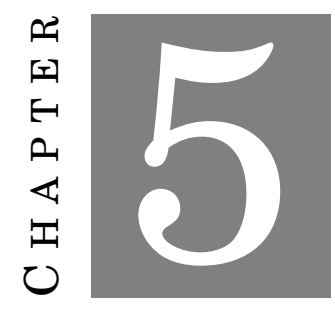

Aspergillus niger MEMBRANE-ASSOCIATED PROTEOME ANALYSIS FOR THE IDENTIFICATION OF GLUCOSE

TRANSPORTERS

\section{This chapter is adapted from:}

Sloothaak J*, Odoni DI*, de Graaff LH, Martins dos Santos VAP, Schaap PJ, Tamayo-Ramos JA. (2015). Aspergillus niger membrane-associated proteome analysis for the identification of glucose transporters. In Biotechnology for biofuels (https://biotechnologyforbiofuels.biomedcentral.com/articles/10.1186/s13068015-0317-9).

*equal contributions 


\subsection{Abstract}

Background: The development of biological processes that replace the existing petrochemical based industry is one of the biggest challenges in biotechnology. Aspergillus niger is one of the main industrial producers of lignocellulolytic enzymes, which are used in the conversion of lignocellulosic feedstocks into fermentable sugars. Both the hydrolytic enzymes responsible for lignocellulose depolymerisation and the molecular mechanisms controlling their expression have been well described, but little is known about the transport systems for sugar uptake in A. niger. Understanding the transportome of $A$. niger is essential to achieve further improvements at strain and process design level. Therefore, this study aims to identify and classify $A$. niger sugar transporters, using newly developed tools for in silico and in vivo analysis of its membrane-associated proteome.

Results: In the present research work, a hidden Markov model (HMM), that shows a good performance in the identification and segmentation of functionally validated glucose transporters was constructed. The model $\left(\mathrm{HMM}_{\mathrm{gluT}}\right)$ was used to analyse the $A$. niger membrane-associated proteome response to high and low glucose concentrations at a low $\mathrm{pH}$. By combining the abundance patterns of the proteins found in the $A$. niger plasmalemma proteome with their $\mathrm{HMM}_{\text {gluT }}$ scores, two new putative high affinity glucose transporters, denoted MstG and MstH, were identified. MstG and MstH were functionally validated and biochemically characterised by heterologous expression in a $S$. cerevisiae glucose transport null mutant. They were shown to be a high affinity glucose transporter $(\mathrm{Km}=0.6$ $\pm 0.1 \mathrm{mM})$ and a very high affinity glucose transporter $(\mathrm{Km}=0.06 \pm 0.005 \mathrm{mM})$ respectively.

Conclusions: This study, focusing for the first time on the membrane-associated proteome of the industrially relevant organism $A$. niger, shows the global response of the transportome to the availability of different glucose concentrations. Analysis of the A. niger transportome with the newly developed $\mathrm{HMM}_{\text {gluT }}$ showed to be an efficient approach for the identification and classification of new glucose transporters. 


\subsection{Background}

The development of biological systems for the industrial synthesis of biofuels and chemicals is a main objective of today's biotechnology. For cost-effective production of valuable products, efficient microbial fermentations of non-food lignocellulosic material are essential. Filamentous fungi, in particular the fungus Aspergillus niger, play a prominent role in this field of biotechnology. A. niger is of significant industrial relevance and has been exploited as a production platform for both organic acids and hydrolytic enzymes (Andersen et al., 2011). It is an efficient degrader of the major plant cell wall polysaccharides cellulose, hemicellulose and pectin (de Souza et al., 2013), and is one of the main industrial producers of commercial enzymes for plant biomass conversion due to its high enzyme secretory capacity (Tamayo-Ramos and Orejas, 2014). In the last decades, its versatile arsenal of extracellular enzymes for lignocellulose depolymerisation (de Vries and Visser, 2001), and the molecular mechanisms controlling their expression, have been well described (Battaglia et al., 2011; Gruben et al., 2014; van Peij et al., 1998). However, while previous studies have revealed the existence of an array of uptake systems in this fungus (Coelho et al., 2013; Jørgensen et al., 2007; Torres et al., 1996), little is known about the identity and specificity of the transport systems involved in sugar uptake.

Most of the existing knowledge related to monosaccharide uptake in fungi originates from studies in the model yeast Saccharomyces cerevisiae. This yeast is able to transport and metabolise glucose, fructose, mannose and galactose. Transport of these simple sugars is mediated only through facilitated diffusion by the majority of the transporters from the Hxt family, composed of Hxt1p-Hxt17p, and Gal2p. They belong to the sugar porter (SP) family (Leandro et al., 2009), which is the largest subfamily of the major facilitator superfamily (MFS). Hxt1Hxt4, Hxt6 and Hxt7 have been found to be able to support growth of yeast in glucose on their own, thus being considered the major hexose transporters in yeast. In addition to the Hxt family, three members of the maltose transporter family (Agt1, Mph2 and Mph3) are also able to transport glucose (Wieczorke et al., 1999). The individual characterisation of each of these transporters was possible using engineered $S$. cerevisiae strains, deleted for hxt1-7 (Reifenberger et al., 1995) and hxt1-17, gal2, agt1, mph2 and mph 3 (Wieczorke et al., 1999), as hosts for the functional validation and biochemical study of these proteins. These yeast mutant strains, unable to grow on glucose, fructose, mannose and galactose as a single carbon source, have also subsequently been used as tools for the functional characterisation of sugar transporters from other fungal species 
(Colabardini et al., 2014; dos Reis et al., 2013; Du et al., 2010; Leandro et al., 2013; Polidori et al., 2007; Saloheimo et al., 2007; Vankuyk PA et al., 2004; Wahl et al., 2010)

In contrast to $S$. cerevisiae, the only functionally validated sugar transporters in A. niger are the recently identified D-galacturonic acid transporter GatA (Martens-Uzunova and Schaap, 2008; Sloothaak et al., 2014), two fructose transporters (Coelho et al., 2013) and the high-affinity sugar $/ \mathrm{H}^{+}$symporter MstA (Vankuyk PA et al., 2004). Furthermore, transcriptional data for the A. niger mstC gene suggests that it encodes a low-affinity glucose transporter (Jørgensen et al., 2007), but no experimental data supporting its role as a functional sugar transporter is publicly available. MFS proteins display a strong structural conservation (Vardy et al., 2004), and structure based profile hidden Markov models can be used to identify putative sugar transporters in the A. niger in silico proteome. To obtain a profile hidden Markov model (HMM), a multiple sequence alignment is turned into a position-dependent scoring system with segments of variable conservation levels and length (Eddy, 1998). As a result of the weighted scoring system, HMMs are less sensitive to changes in the non-conserved regions of a given protein family than more traditional methods based on shared primary sequence similarity alone, like e.g. the standard Blast algorithm. These changes include variability of the residues at a certain position as well as insertions and deletions. In this study, a profile hidden Markov model specific for glucose transporters $\left(\mathrm{HMM}_{\mathrm{gluT}}\right)$ was computed based on a structure-based multiple sequence alignment of 42 proteins with a known function related to glucose transport.

Genome information combined with transcriptome analysis of various growth conditions can give a good inventory of $A$. niger plasma membrane components with hypothetical sugar transporter functions. These data types, however, by definition do not take regulatory events at the post-transcriptional level into account, although these events can influence protein abundances and localisation. An inventory of the A. niger plasma membrane proteome at defined culture conditions can provide a more reliable source of information for the identification of the most important sugar transport components. To date, only few fungal plasmalemma (PM) enriched proteomes have been reported, and none of them involved an industrially relevant filamentous fungus. The main focus of study has been on S. cerevisiae (Delom et al., 2006; Kim et al., 2006; Szopinska et al., 2011) and on several pathogenic fungi (Cabezon et al., 2009; Ouyang et al., 2010; Rogers et al., 2006), as their PM represents a cellular component of substantial interest from a diagnostic and therapeutic point of view (Cabezon et al., 2009). Thus, since the PM proteome of $A$. niger has not been a subject of study yet, it 
would be a first step towards a better understanding of its dynamics and topology.

Recently, our research group successfully used a shotgun proteomics approach to study protein secretion mechanisms in A. niger, allowing the characterisation of the secretory subproteome of the fungus and its changes in different conditions by using label-free LC-MS/MS (de Oliveira et al., 2010, 2011). This is a powerful tool to analyse enriched organelle cell fractions, both in qualitative and quantitative terms (Patel et al., 2009), and thus permits the identification and quantification of the most relevant components of the A. niger cell membranes. In this work, the membrane-associated proteome of $A$. niger was studied for the identification of new glucose transporters, using newly developed experimental and complementary computational approaches.

\subsection{Results and discussion}

\subsubsection{In silico transportome analysis and construction of a hidden Markov model specific for glucose transporters}

As an integral part of the membrane, transporters must contain at least one protein domain that is thermodynamically stable in the hydrophobic environment of the phospholipid tails. In eukaryotes, these are typically $\alpha$-helical structures. Transmembrane proteins can be predicted by applying a transmembrane hidden Markov model (tmHMM) that incorporates the hydrophobicity, charge bias, helix lengths and grammatical constraints of known transmembrane proteins into one model (Krogh et al., 2001). In addition, a comprehensive list of A. niger sugar transporters can be made by applying hidden Markov models for both the major facilitator superfamily $\left(\mathrm{HMM}_{\mathrm{MFS}}\right)$, and sugar porters $\left(\mathrm{HMM}_{\mathrm{SP}}\right)$. As the largest subfamily of the MFS, the sugar porter (SP) family currently has the most identified members (Pao et al., 1998), and as such provides a good basis for constructing a specific profile HMM from multiple alignments of extensively characterised members.

The experimental steps and complementary bioinformatics pipeline to identify and validate $A$. niger glucose transporters is depicted in Figure 5.1. Initial in silico analysis of the theoretical $A$. niger proteome with the precomputed $\mathrm{HMM}_{\mathrm{MFS}}$ and $\mathrm{HMM}_{\mathrm{SP}}$, obtained from the Pfam database (Punta et al., 2011), showed that more than 250 of the proteins predicted in A. niger ATCC 1015 have a conserved domain architecture related to sugar transport (Table 5.1). Very similar results were obtained for the A. niger CBS 513.88 strain (not shown). This high abundance of sugar transporters makes $A$. niger a versatile host for 
the bioconversion of lignocellulosic biomass to products of interest, especially also in comparison to $S$. cerevisiae, which cannot metabolise as many sugars and consequently has fewer sugar transporters (Leandro et al., 2009). Although the $\mathrm{HMM}_{\mathrm{SP}}$ captures the conserved domain architecture of potential sugar porters, it cannot discriminate between different sugar substrates, and was thus considered to be too broad for the purpose of this study. Therefore, a list of 42 biochemically characterised glucose transporters from 10 different organisms was obtained from the UniProt database (Consortium et al., 2014), and a profile HMM specific for glucose transporters $\left(\mathrm{HMM}_{\text {gluT }}\right)$ was built from these sequences (see alignment in additional file 1). The functions of proteins are often more conserved in their tertiary structure than in their primary amino acid sequence, as residues that are not crucial for the function of the protein will be subject to evolutionary changes over time. The typical structure of known glucose transporters comprises 12 transmembrane helix domains divided into 2 groups of 6 helixes (Deng et al., 2014; Zeng et al., 1996), and the $\mathrm{HMM}_{\text {gluT }}$ was computed from a structure based multiple sequence alignment incorporating transmembrane helix predictions, rather than using an alignment algorithm based on the primary amino acid sequence alone. Appropriate threshold values, above which a hit with the HMM $\mathrm{HluT}_{\mathrm{T}}$ has to score in order to be considered a true positive, can be calculated by evaluating the properties of the $\mathrm{HMM}_{\text {gluT }}$. Receiver operating characteristic (ROC) curves are instrumental in assigning the best threshold values, since they display the trade-off between sensitivity and specificity. In order to obtain ROC curves, the $\mathrm{HMM}_{\text {gluT }}$ was first validated using a $10 \times 3$-fold cross validation approach (Figure 5.2). Threshold values with the best trade-off between the true and false positive rates of the prediction were determined from the resulting ROC curve. In this study, two thresholds were used. The first is $\mathrm{d}_{\min }$, which is the point on the curve that has the minimal distance to [0 1] in the 2-dimensional x-y plane. Another way to determine the best trade-off point is by calculating the point in which the Matthews correlation coefficient is maximal $\left(\mathrm{MCC}_{\max }\right)$, as the prediction is regarded as better the closer the MCC value is to 1 , whereas a value of 0 is regarded as no better than a random guess. To compare the performance of this approach to a more traditional approach, in which Blast is used to identify homologous proteins, each of the 42 verified glucose transporters was used as query in a separate Blast search against the same dataset used to evaluate the HMM $\mathrm{Hlu}_{\text {glu }}$. The resulting ROC curves for each of the methods is depicted in additional file 2 and shows that, for the datasets used, an approach using HMMs outperforms Blast in discriminating glucose transporters from other sugar transporters.

By using the previously calculated thresholds as lower limits for the $\mathrm{HMM}_{\mathrm{gluT}}$ 


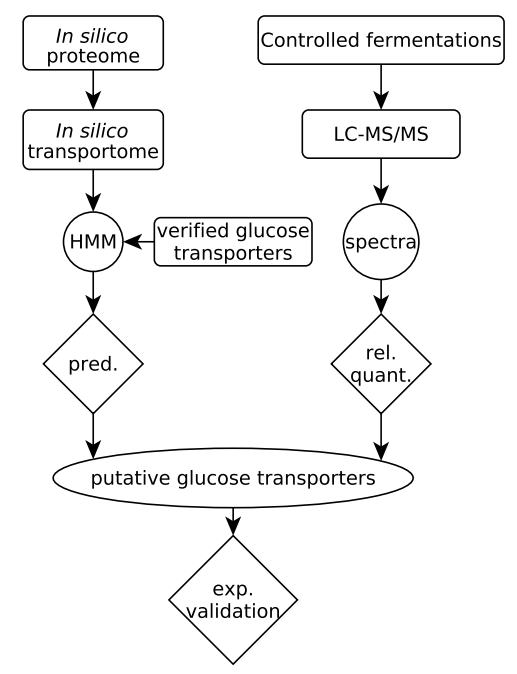

FIGURE 5.1. Bioinformatic and proteomic profiling of the A. niger transportome. The flow chart outlines the complementary approach taken to identify putative glucose transporters. Experimental validation of the transporters identified was carried out in $S$. cerevisiae.

Table 5.1: MFS domain proteins in A. niger and S. cerevisiae. $\mathrm{N}_{\text {tot }}=$ total number of predicted proteins; $\mathrm{N}_{\mathrm{tmHMM}}=$ number of proteins containing at least one transmembrane helix domain; $\mathrm{N}_{\mathrm{MFS}}=$ number of proteins containing a MFS domain; $\mathrm{N}_{\mathrm{SP}}=$ number of proteins containing a SP domain; $\mathrm{N}_{\text {gluT }}=$ number of proteins above inclusion threshold of $\mathrm{HMM}_{\text {gluT }}$ (as given by the HMMER3.0 tool); $\mathrm{N}_{\text {gluT }}\left(\mathrm{d}_{\min }\right)=$ number of proteins above the inferred threshold score at $\mathrm{d}_{\min } ; \mathrm{N}_{\text {gluT }}\left(\mathrm{MCC}_{\max }\right)=$ number of proteins above the inferred threshold score at $\mathrm{MCC}_{\max }$

\begin{tabular}{lccccccc}
\hline & $\mathrm{N}_{\text {tot }}$ & $\mathrm{N}_{\text {tmHMM }}$ & $\mathrm{N}_{\mathrm{MFS}}$ & $\mathrm{N}_{\mathrm{SP}}$ & $\mathrm{N}_{\text {gluT }}$ & $\mathrm{N}_{\text {gluT }}\left(\mathrm{d}_{\min }\right)$ & $\mathrm{N}_{\text {gluT }}\left(\mathrm{MCC}_{\max }\right)$ \\
\hline A. niger ATCC1015 & 11910 & 2561 & 469 & 256 & 252 & 19 & 5 \\
S. cerevisiae CEN.PK & 5439 & 1022 & 73 & 43 & 45 & 15 & 12 \\
\hline
\end{tabular}

score, the list of putative glucose transporters was effectively narrowed down to 19 sugar porters when the $d_{\min }$ threshold was applied, and to only 5 sugar porters, including MstA, when the $\mathrm{MCC}_{\max }$ threshold was applied (Table 5.1). Note that MstA was truly "discovered" by the $\mathrm{HMM}_{\text {gluT }}$, since it was not a priory included in the training set. The average sensitivity, specificity, accuracy and inferred $\mathrm{HMM}_{\text {gluT }}$ scoring values at the two threshold points, $\mathrm{d}_{\min }$ and $\mathrm{MCC}_{\max }$, are noted in Table 5.2. As expected, the specificity at the MCC $_{\max }$ threshold is very high, but it comes at the cost of having a lower sensitivity. The $d_{\min }$ threshold on the 


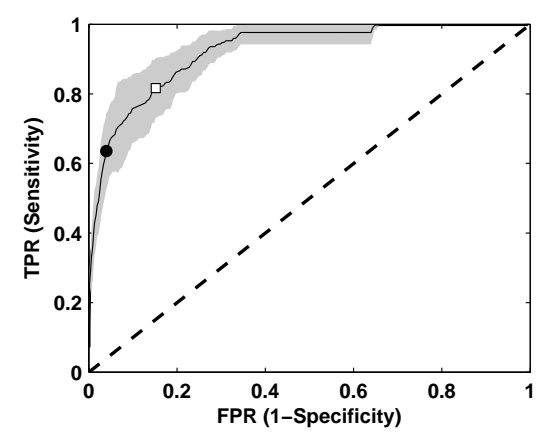

FIGURE 5.2. Receiver operating characteristic (ROC) curve for the HMM $_{\text {glut }}$ transporter model. Plotted is the mean of 30 runs of a $10 \times 3$-fold cross-validation. The confidence interval is shown in grey. Calculated inclusion thresholds, $d_{\min }$ (white square) and $\mathrm{MCC}_{\max }$ (black circle), are indicated

other hand is less strict, thus allowing for a higher false positive rate in order to not neglect any true positives.

Table 5.2: Average sensitivity, specificity, accuracy and inferred $\mathrm{HMM}_{\text {gluT }}$ threshold values for the $\mathrm{HMM}_{\text {gluT }}$ at $\mathrm{d}_{\min }$ and $\mathrm{MCC}_{\max }$.

\begin{tabular}{lcccc}
\hline & Sensitivity [\%] & Specificity [\%] & Accuracy [\%] & HMM $_{\text {gluT }}$ score \\
\hline $\mathrm{d}_{\min }$ & 81.67 & 84.79 & 84.61 & 379.41 \\
$\mathrm{MCC}_{\max }$ & 63.57 & 96.00 & 94.12 & 531.03 \\
\hline
\end{tabular}

\subsubsection{The A. niger plasmalemma proteome in response to different glucose concentrations at low $\mathbf{p H}$}

A way to verify both the $\mathrm{HMM}_{\text {gluT }}$ and the predicted glucose transporter candidates is by using an experimental set up, in which the abundance of transmembrane proteins in relevant conditions can be compared with their $\mathrm{HMM}_{\mathrm{gluT}}$ scores. In this complementary work, the focus was put on the study of the $A$. niger transportome in the presence of different concentrations of glucose at a $\mathrm{pH}$ which is both physiologically and biotechnologically relevant for this fungus. Mycelium of A. niger N400 was pre-grown for $18 \mathrm{~h}$ in minimal medium with $100 \mathrm{mM}$ sorbitol as sole carbon source and subsequently transferred in equal amounts to controlled fermenters containing minimal medium with the following carbon source compositions: $100 \mathrm{mM}$ sorbitol (reference condition), $100 \mathrm{mM}$ sorbitol plus $1 \mathrm{mM}$ glucose (low-glucose condition) and $100 \mathrm{mM}$ sorbitol plus 60 $\mathrm{mM}$ glucose (high-glucose condition). The initial $\mathrm{pH}$ of these cultures was set 
at $\mathrm{pH} 4.0$, which corresponded with the final $\mathrm{pH}$ measured at the pre-growth stage. Immediately after inoculation the $\mathrm{pH}$ of the fermenter cultures started to drop, reaching $\mathrm{pH} 3.5$, in all cases, after 50 to $60 \mathrm{~min}$. For the remaining of the experiment this was kept constant at $\mathrm{pH}$ 3.5. High resolution analysis of the sugar content in the culture medium sampled two hours after inoculation showed that there was sorbitol consumption in the reference condition; sorbitol and glucose consumption in the low-glucose condition; and glucose but no sorbitol consumption in the high-glucose condition. This result confirmed, as reported (Strauss et al., 1999), that the organism strongly favours consumption of good carbon sources like glucose, over poorer carbon sources, such as sorbitol, even when the latter is also present at a high concentration (100 $\mathrm{mM})$. The two-hour time-point was selected for membrane associated protein analysis in all three conditions.

Isolation of fractions enriched for cell membranes was performed using a protocol similar to the one developed by Oliveira and co-workers (de Oliveira et al., 2010). This protocol involves the previously described workflow: cell disruption, crude organelle separation, and subsequent enrichment (de Oliveira and de Graaff, 2011). After several differential centrifugation steps, a pellet containing crude low-density organelles (P3) was obtained and subjected to density gradient centrifugation, yielding a set of five fractions (P3A to P3E). The PM marker vanadate-sensitive $\mathrm{H}^{+}$ATPase and the mitochondrial membrane cytochrome c oxidase activities were then measured in the initial cell free extract, the P3 pellet and the P3A to P3E fractions derived from it. Compared to the cell free extract the P3 pellet was shown to be 2.4 to 3.2 times enriched in plasma membranes. No further enhanced PM enrichment was found in the P3A to P3E fractions, however, cytochrome c oxidase activity was higher in the P3A to P3E fractions when compared to P3. Since mitochondrial membranes were not the main focus of this research, the P3 pellets, considered to be more optimal for the analysis of plasmalemma proteins, were further processed and subjected to shotgun proteomics analysis (detailed information regarding subcellular fractionation, marker enzyme assays and sample preparation for LC-MS/MS can be found in the Methods section).

The LC-MS/MS spectra obtained were processed as described in the Methods section. In total, 833 proteins were identified, of which 432 were present in all three conditions, 72 proteins were found only in the reference condition, 34 only in the low-glucose condition, and 106 proteins were found exclusively in the high-glucose condition. Of the proteins identified, almost $30 \%$ had one or more predicted tmHMM domains, indicative of integral membrane proteins (additional 
file 3). The relative abundances of the proteins containing at least one tmHMM domain, which add up to $15.28 \%, 17.50 \%$ and $15.45 \%$ of the total protein isolated in the reference, low- and high-glucose conditions, respectively, can be found in additional file 4 . Proteins associated with the mitochondrial membrane were most abundant, followed by the proteins associated with the plasma membrane. However, the plasma membrane-associated fraction consists of a higher number of different proteins than the mitochondrial membrane-associated fraction, with 86 and 35 identified proteins, respectively. Proteins associated with the membrane bound endoplasmic reticulum (ER) constitute the third most abundant group of the list, with a total of 48 different proteins identified. Finally, proteins linked to other membrane-associated organelles, comprising the endomembrane system and membrane bound organelles, such as the Golgi apparatus, vacuoles and lysosomes, account for $<1 \%$ of the total protein isolated (additional file 5). In all three conditions, approximately one third of the proteins that have one or more tmHMM domains are annotated as proteins with a transporter function. This fraction, denoted as the A. niger transportome, accounted for $3.9 \%, 4.4 \%$ and $4.1 \%$ of the total protein isolated in the reference, low- and high-glucose conditions, respectively. Mitochondrial carrier proteins are prevalent in all three conditions, followed by ATPases. Proteins with the MFS architecture, which are the main interest in this study, comprise the third most abundant group of the isolated A. niger transportome. Proteins (putatively) involved in the secretory pathway and amino acid transport were found in relative abundances of $<0.5 \%$ of the total protein isolated. Other transporters, comprising (putative) oligopeptide transporters, formate/nitrite transporters, ammonium transporters, iron permeases, $\mathrm{Na}^{+}$/solute symporters, $\mathrm{ABC}$ transporters, inorganic phosphate transporters, nucleotide-sugar transporters, major intrinsic proteins and the translocation protein Sec62, accounted for $\leq 0.01 \%$ of the total protein isolated, respectively.

\subsubsection{Performance of the $\mathrm{HMM}_{\text {gluT }}$ for the identification of candidate glucose transporters}

The A. niger transportome was further analysed for the presence of glucose transporters. Table 5.3 summarises the results of the bioinformatics analysis, which was performed in the same way as previously for the entire A. niger in silico proteome, i.e. the data were first queried with the hidden Markov models specific for proteins containing a MFS or SP domain. As already observed for the A. niger in silico proteome, a search with the $\mathrm{HMM}_{\mathrm{SP}}$ lowered the number of 

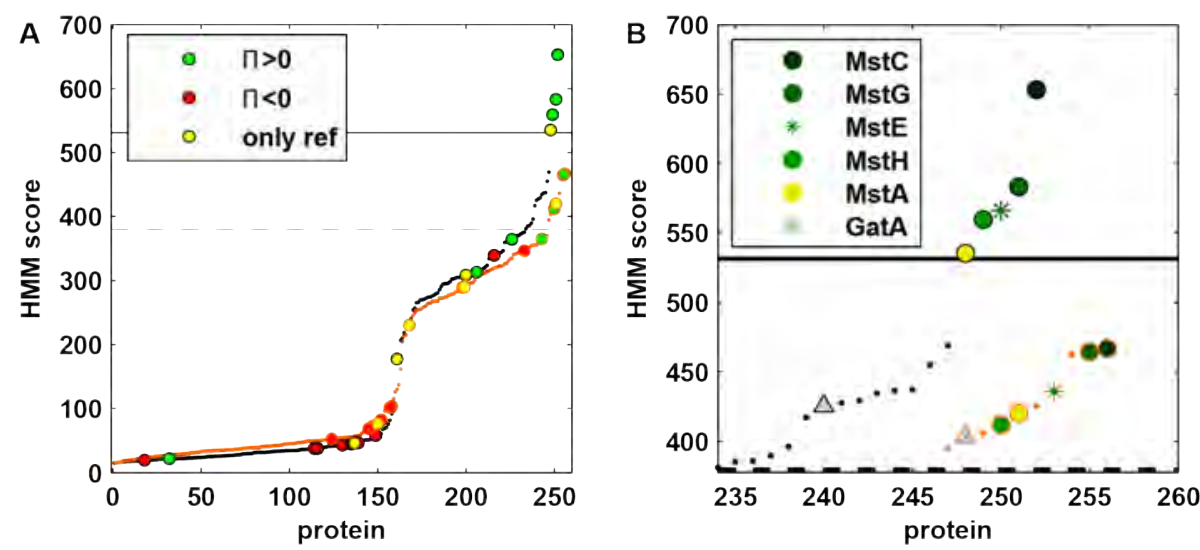

FIGURE 5.3. HMM SP $_{\text {and }}$ HMM $_{\text {gluT }}$ scores of MFS porter proteins.

A) HMM scores for the theoretical ATCC 1015 proteome, with $\mathrm{HMM}_{\mathrm{gluT}}=$ black dotted line and $\mathrm{HMM}_{\mathrm{SP}}=$ orange dotted line. Horizontal lines indicate the $\mathrm{HMM}_{\text {gluT }}$ thresholds $\mathrm{d}_{\text {min }}$ (dashed) and $\mathrm{MCC}_{\max }$ (solid). MFS porter proteins found in the three conditions applied are indicated with filled circles and colour-coded according to their relative abundance patterns. B) Close-up of the proteins that scored above the $\mathrm{d}_{\min }$ (dashed line) and $\mathrm{MCC}_{\max }$ (solid line) thresholds. The galacturonic acid transporter GatA (indicated with a grey triangle and not found in the conditions applied) is added as a reference. With $\mathrm{HMM}_{\text {gluT }}$, the Mst transporters are well separated from GatA, whereas the more general $\mathrm{HMM}_{\mathrm{SP}}$ does not discriminate the putative glucose transporters from the galacturonic acid transporter

proteins that scored above the inclusion threshold in comparison to the search performed with the less specific $\mathrm{HMM}_{\mathrm{MFS}}$. Querying the transportome with the newly developed $\mathrm{HMM}_{\text {gluT }}$, using the previously calculated thresholds, lowered the number of putative, albeit highly probable, glucose transporters to 4,3 , and 2 promising hits in the reference, low- and high-glucose conditions, respectively.

The relative abundances of the identified MFS proteins that scored above the default inclusion threshold of $\mathrm{HMM}_{\text {gluT }}, \mathrm{HMM}_{\mathrm{SP}}$ and $\mathrm{HMM}_{\mathrm{MFS}}$ ordered by their $\mathrm{HMM}_{\text {gluT }}$ scores, can be found in Table 5.4. High HMM scores corresponded to MFS porters that have been related to glucose uptake in previous studies, or novel MFS porters that, in the present study, showed abundance patterns that point towards a possible role as glucose transporter. In Figure 5.3A the proteins found in the three conditions are highlighted on the HMM curves for the glucose and general sugar transporter model depicted. The colour coding corresponds 
Table 5.3: Number of MFS porter proteins found in the 3 growth conditions. $\mathrm{N}_{\text {MFS }}$ = number of proteins containing a MFS_1 domain; $\mathrm{N}_{\mathrm{SP}}=$ number of proteins containing a SP domain; $\mathrm{N}_{\text {gluT }}=$ number of proteins above the default inclusion threshold of $\mathrm{HMM}_{\text {gluT }}$ (as given by the HMMER3.0 tool); $\mathrm{N}_{\text {gluT }}\left(\mathrm{d}_{\min }\right)=$ number of proteins above inferred threshold score at $\mathrm{d}_{\min } ; \mathrm{N}_{\text {gluT }}\left(\mathrm{MCC}_{\max }\right)=$ number of proteins above inferred threshold score at $\mathrm{MCC}_{\max }$

\begin{tabular}{lccccc}
\hline & $\mathrm{N}_{\mathrm{MFS}}$ & $\mathrm{N}_{\mathrm{SP}}$ & $\mathrm{N}_{\text {gluT }}$ & $\mathrm{N}_{\text {gluT }}\left(\mathrm{d}_{\min }\right)$ & $\mathrm{N}_{\text {gluT }}\left(\mathrm{MCC}_{\max }\right)$ \\
\hline sorbitol & 14 & 13 & 13 & 4 & 4 \\
sorbitol + 1mM glucose & 9 & 6 & 7 & 3 & 3 \\
sorbitol + 60mM glucose & 15 & 9 & 10 & 2 & 2 \\
\hline
\end{tabular}

to their relative abundances in the three conditions (see Methods for details). The HMM curves as such show the scores of all proteins in the theoretical $A$. niger ATCC1015 proteome that hit above the default inclusion threshold of both $\mathrm{HMM}_{\mathrm{gluT}}$ and $\mathrm{HMM}_{\mathrm{SP}}$. The majority of proteins that were more abundant in the low-glucose condition relative to both the reference and high-glucose condition cluster closer to the $d_{\min }$ and $\mathbf{M C C}_{\max }$ thresholds, whereas the proteins that were more abundant in the high-glucose condition scored overall lower on the $\mathrm{HMM}_{\text {gluT }}$. This indicates that the model is better at detecting high-affinity glucose transporters, which might be due to the sequences that were selected to build the $\mathrm{HMM}_{\text {gluT }}$ (additional file 1). A close-up of the top scoring proteins is depicted in Figure 5.3B. The highest score was obtained with protein ID 1121621, which is the putative low-affinity glucose transporter MstC. Studies on its transcriptional regulation during the exponential growth phase in batch fermentation and in chemostat cultures, carried out at different dilution rates, have been performed by Jørgensen et al. (2007). The $m s t C$ transcripts were only detected during the batch fermentation phase, indicating that $m s t C$ expression is associated with higher glucose concentrations or with specific growth rates. In the present work, the protein abundance of the MstC protein was found to be similar in all the three conditions studied. This result, together with the previous findings of Jørgensen et al., suggests that the presence of MstC is independent of the glucose concentration. The second best hit, with protein ID 1142882, and the fourth best hit, with protein ID 1143598, are yet-uncharacterised transport proteins, henceforth denoted as MstG and MstH, respectively. Their high $\mathrm{HMM}_{\text {gluT }}$ scores and overall abundance pattern in the three experimental conditions strongly indicate a possible role for them as glucose transporters. MstG and MstH were found with relatively high abundance levels only in the two non carbon catabolite-repressing conditions studied, being higher in abundance when low glucose concentrations were present. This type of abundance pattern fits that of high affinity glucose transporters 
described in S. cerevisiae and Aspergillus species (Forment et al., 2014; Özcan and Johnston, 1999; Vankuyk PA et al., 2004), that are preferably expressed in the presence of low concentrations of glucose, poor carbon (de-repressing) sources and also in starvation conditions. Another protein with protein ID 1125134, scoring in between MstG and MstH in the $\mathrm{HMM}_{\text {gluT }}$ was not observed in the three studied conditions. It is denoted as MstE (An03g02190) in the UniProt database, and has been found to be expressed in germinating spores (Van Leeuwen et al., 2013). The fifth highest scoring hit, with protein ID 1143191, is MstA. The $m s t A$ coding gene is transcriptionally controlled by the carbon catabolite repressor CreA and the environmental $\mathrm{pH}$ regulator PacC. Its transcript levels were found to be higher during carbon starvation at pH 6.0 (Vankuyk PA et al., 2004). Van Kuyk et al. also observed low expression levels at $\mathrm{pH} 4.0$ and 8.0, and in the presence of repressing glucose concentrations at $\mathrm{pH} 6.0$ (Vankuyk PA et al., 2004). In the present study, MstA was detected in very low abundance, however only in the de-repressing reference condition, supporting previous results that suggested a limited role of this transporter when the environmental $\mathrm{pH}$ is low. The combined results of the specific protein abundance patterns and the high $\mathrm{HMM}_{\mathrm{gluT}}$ scores indicate that MstG and MstH could have a role as high/mid affinity glucose transporters, therefore both transporters were selected for further characterisation.

Table 5.4: Relative abundance and HMM scores of MFS porter proteins detected in the three growth conditions. UniProt accession numbers in brackets; n.d: not detected in one or both of the biological replicates; *these proteins could not be distinguished by the proteomics analysis and were thus grouped together to one protein group.

\begin{tabular}{|c|c|c|c|c|c|c|c|}
\hline \multirow[b]{2}{*}{ protID } & \multirow[b]{2}{*}{$\mathrm{HMM}_{\text {gluT }}$} & \multirow[b]{2}{*}{$\mathrm{HMM}_{\mathrm{SP}}$} & \multirow[b]{2}{*}{$\mathrm{HMM}_{\mathrm{MFS}}$} & \multicolumn{3}{|c|}{ Relative abundance \pm sd $[\%] 100$} & \multirow[t]{2}{*}{ Remark } \\
\hline & & & & sorbitol & $\begin{array}{c}\text { sorbitol + } \\
\text { 1mM glucose }\end{array}$ & $\begin{array}{c}\text { sorbitol + } \\
60 \mathrm{mM} \text { glucose }\end{array}$ & \\
\hline 1121621 & 653.1 & 467 & 78.5 & $9.08 \pm 1.02$ & $11.89 \pm 4.79$ & $9.47 \pm 3.50$ & MstC (Q8J0U9) \\
\hline 1142882 & 583.1 & 464.5 & 74.8 & $2.24 \pm 0.48$ & $4.47 \pm 0.20$ & n.d. & MstG \\
\hline 1143598 & 559.3 & 411.7 & 86.1 & $6.38 \pm 1.83$ & $17.54 \pm 2.39$ & n.d & MstH \\
\hline 1143191 & 535 & 420 & 75.7 & $8.51 \mathrm{e}-3 \pm 1.14 \mathrm{e}-3$ & n.d. & n.d. & MstA (Q8J0V1) \\
\hline 1101809 & 364.4 & 364.7 & 85.6 & $4.83 \pm 0.51$ & $2.01 \pm 0.29$ & n.d. & \\
\hline 1180703 & 339.2 & 346.3 & 97.4 & n.d. & n.d. & $1.37 \pm 0.14$ & mstD (Q8J0U8) \\
\hline 1188093 & 312.9 & 289.3 & 82.0 & $1.08 \pm 0.39$ & $0.82 \pm 0.11$ & $0.82 \pm 0.25$ & \\
\hline 1144791 & 308.1 & 289.6 & 55.9 & $6.25 \pm 1.25$ & n.d. & n.d. & \\
\hline 1189214 & 177.3 & 230.6 & 73.7 & $0.29 \pm 0.03$ & n.d. & n.d. & \\
\hline 1128338 & 57.6 & 103.6 & 72.9 & $3.70 \pm 0.32$ & $4.36 \pm 0.02$ & $6.46 \pm 0.84$ & \\
\hline 1111630 & 46.4 & 101.6 & 63.2 & n.d. & n.d. & $1.06 \pm 0.10$ & \\
\hline 1184634 & 46 & 76.4 & 0 & $0.30 \pm 4.12 \mathrm{e}-5$ & n.d. & n.d. & \\
\hline 1122202 & 44.6 & 52.6 & 135.9 & $0.87 \pm 0.10$ & n.d. & $0.48 \pm 0.29$ & \\
\hline 1178623 & 42.9 & 68.3 & 149.3 & n.d. & n.d. & $4.71 \pm 1.07$ & \\
\hline $\begin{array}{l}1164538 ; \\
1188786\end{array}$ & $37.5 ; 37.4$ & $82.3 ; 72.1$ & $104.3 ; 98.9$ & $2.11 \pm 0.14$ & n.d. & $2.60 \pm 0.21$ & $\begin{array}{l}\text { same protein } \\
\text { group* }\end{array}$ \\
\hline 1089440 & 21.4 & 0 & 92.5 & n.d. & $1.16 \pm 0.12$ & n.d. & \\
\hline 1118545 & 19.1 & 0 & 0 & n.d. & n.d. & $3.87 \pm 1.13$ & \\
\hline 1105147 & 0 & 0 & 54.4 & $8.93 \pm 0.03$ & $7.14 \pm 2.01$ & $11.47 \pm 1.24$ & \\
\hline 1129336 & 0 & 0 & 31.8 & $0.34 \pm 0.01$ & $0.50 \pm 0.10$ & $1.07 \pm 0.01$ & \\
\hline 1124902 & 0 & 0 & 130.7 & n.d. & n.d. & $1.37 \pm 0.44$ & \\
\hline 1165706 & 0 & 0 & 114.3 & n.d. & n.d. & $0.05 \pm 0.01$ & \\
\hline 1188840 & 0 & 0 & 99.1 & n.d. & n.d. & $3.00 \pm 1.30$ & \\
\hline 1146101 & 0 & 0 & 65.3 & n.d. & n.d. & $1.21 \pm 0.91$ & \\
\hline
\end{tabular}




\subsubsection{Transcriptional analysis of $m s t G$ and $m s t H$ in the presence of different carbon sources}

In order to obtain additional insights in the regulation and possible biological role of MstG and MstH, transcriptional levels were analysed in mycelium samples from cultures containing: minimal medium with $100 \mathrm{mM}$ sorbitol (used as a reference condition) and minimal medium plus glucose, fructose or mannose at high $(55 \mathrm{mM})$ or low $(5 \mathrm{mM})$ concentration. Samples taken two hours after mycelium transfer where processed and RT-qPCR analysis was performed. Both genes were found to be expressed in all the studied conditions, but their expression levels were clearly influenced by the different culture conditions (Figure 5.4). The relative transcript levels of both genes in the reference, low-glucose and high-glucose conditions showed a pattern comparable to what was observed at proteomic level; $m s t G$ and $m s t H$ were upregulated in the presence of a low glucose concentration and downregulated when the glucose concentration was high. However, $m s t G$ and $m s t H$ expression patterns showed to be divergent in the presence of high and low concentrations of fructose and mannose. With these carbon sources $m s t G$ expression levels were similar to those observed with a low concentration of glucose. In the case of $m s t H$, as observed in the presence of glucose $55 \mathrm{mM}$, high concentrations of fructose ( $55 \mathrm{mM})$ and mannose (55 mM) also led to a decrease of its transcript levels when compared to the reference condition. While these results do not provide conclusive evidence that MstG is indeed a high affinity glucose transporter they are not in disagreement with its possible role either. In the case of $m s t H$, only the glucose $5 \mathrm{mM}$ condition enhanced its expression, suggesting that MstH could have a specific role in $A$. niger when low concentrations of the monosaccharide are available. 

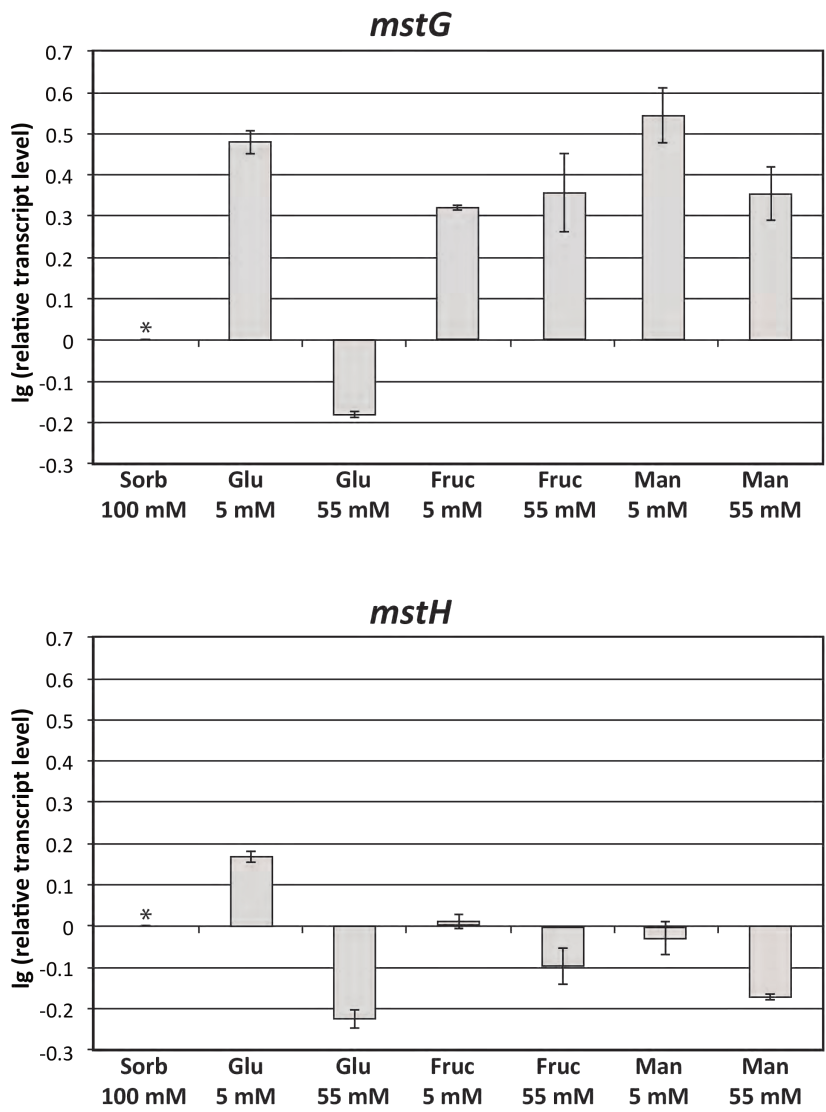

FIGURE 5.4. Transcriptional analysis of mstG and mstH. Mycelium of the $A$. niger strain N400 was precultured on minimal medium with sorbitol $100 \mathrm{mM}$ and thereafter transferred to minimal medium with the following carbon sources: sorbitol $100 \mathrm{mM}$, glucose $5 \mathrm{mM}$, glucose $55 \mathrm{mM}$, fructose $5 \mathrm{mM}$, fructose $55 \mathrm{mM}$, mannose $5 \mathrm{mM}$ and mannose $55 \mathrm{mM}$. Samples from the 7 culture conditions were taken $2 \mathrm{~h}$ after mycelium transfer, and expression analyses of $m s t G$ and $m s t H$ were performed by qPCR using a histone-like gene (gene ID 207921) transcript for normalisation. Results are given as relative transcript rations in logarithmic scale (lg). The values provided in the figures are means of two biological replicates. Transcript levels always refer to the reference sample (sorbitol $100 \mathrm{mM}$ ), indicated with an asterisk. 


\subsubsection{Functional validation of the A. niger sugar transporters MstG and MstH}

As discussed above, the high HMMgluT scores, the protein abundance patterns and transcriptional analyses of the MFS porters MstG and MstH point towards a role in glucose uptake. In order to test their functionality, the engineered $S$. cerevisiae strain EBY.VW.4000 (Wieczorke et al., 1999), a glucose transporter null mutant unable to grow on glucose, mannose, galactose or fructose as carbon source, was chosen as host for functional complementation analysis. The yeast strain was transformed with the $2 \mu$ expression plasmid p426HXT7-6His-mstG or p426HXT7-6His-mstH, containing the respective cDNA under control of the constitutive promoter HXT7p and the terminator CYC1t. Single colony transformants were isolated from minimal medium agar plates containing $2 \%$ maltose and the ability of both genes to restore growth of the EBY.VW.4000 transformant strain in the presence of different monosaccharides was studied. Ten-fold serial dilutions of logarithmically growing cells from at least two different transformants expressing each gene were spotted on different minimal medium plates supplemented with $1 \%(\mathrm{w} / \mathrm{v})$ of the following carbon sources: glucose, galactose, fructose, mannose, sucrose and maltose (Figure 5.5). The $m s t G$ transformants were able to grow on glucose, galactose, mannose and sucrose as single carbon sources, whereas $m s t H$ transformants grew on glucose, sucrose, mannose, galactose and, in contrast to $m s t G$ strains also on fructose. MstH transformants also showed better growth on mannose and sucrose, but poorer growth on galactose. Regarding the sucrose transport by both transporters, since the EBY.VW4000 strain encodes an extracellular invertase (Wahl et al., 2010), it is unknown if the transported substrate was sucrose or glucose in the case of MstG, and sucrose, glucose or fructose in the case of MstH. These results indicated that MstG and $\mathrm{MstH}$ are functional sugar transporters with the ability to transport a variety of substrates. 


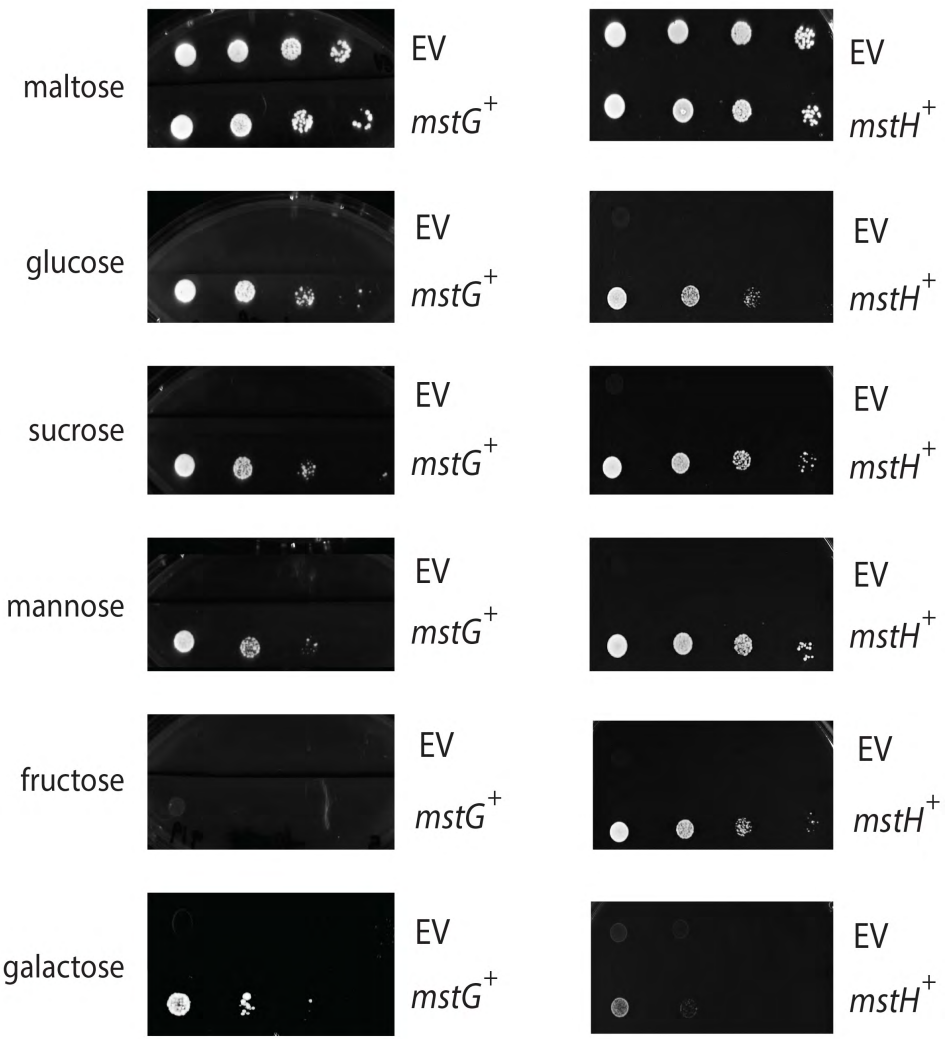

Figure 5.5. MstG and MstH functional analysis. Growth of strain EBY.VW4000 containing the $m s t G$ gene $\left(m s t G^{+}\right)$, $m s t H$ gene $\left(m s t H^{+}\right)$, or harbouring the empty expression vector p426HXT7$6 \mathrm{His}(\mathrm{EV})$ in minimal medium agar plates containing the following sugars at a final concentration of $1 \%(\mathrm{w} / \mathrm{v})$ : maltose, glucose, sucrose, mannose, fructose and galactose. Agar plates were incubated at 30 ${ }^{\circ} \mathrm{C}$ for $96 \mathrm{~h}$. All transformants expressing each gene showed the same growth pattern; therefore the figure depicts only one transformant per transporter as representative.

In order to have better insights about the affinity for glucose of both transporters, the growth rates of the transformants expressing MstG and MstH were studied in the presence of different glucose concentrations. Additional file 6 shows growth curves and glucose consumption figures of the transformant strains grown on minimal medium with glucose $2.5 \mathrm{mM}, 10 \mathrm{mM}$ and $50 \mathrm{mM}$. Both transporters showed to be functional in all glucose concentrations. However, when comparing 
individual growth curves and glucose uptake, some differences could be observed. The growth rate of the MstG transformant during the exponential growth phase was comparable in the three different conditions, while in case of the MstH transformant, at the highest glucose concentration the growth rate was reduced. Accordingly, the glucose consumption rate of the MstG transformant in the 50 $\mathrm{mM}$ condition was much faster, being able to deplete the monosacharide after around 40 hours of growth, whereas in the same time span the MstH transformant was only able to consume a $60 \%$ of the total amount suggesting that MstG and $\mathrm{MstH}$ are glucose transporters with different affinities for the sugar.

To determine MstG and MstH transport characteristics, $\left({ }^{14} \mathrm{C}\right)$ glucose transport assays were performed with the transformant strains as described (Walsh et al., 1994). Initial glucose uptake rates at various substrate concentrations were fitted to the Michaelis-Menten model with non-competitive substrate inhibition and used to estimate the appropriate kinetic parameters as described (Maier et al., 2002). MstG was confirmed to transport glucose with apparent $\mathrm{Km}$-values of $0.5 \pm 0.04 \mathrm{mM}$, and Vmax of $5.8 \pm 0.04 \mathrm{nmol} / \mathrm{min} / \mathrm{mg} \mathrm{DW}$ (Figure 5.6). Its affinity for glucose was lower than the one reported for A. niger MstA with a $\mathrm{Km}$-value of $0.025 \pm 0.01 \mathrm{mM}$. However, MstG can still be classified as a highaffinity glucose transporter, since its $\mathrm{Km}$-value is lower than the one reported for the $S$. cerevisiae high-affinity hexose transporter HXT6 (Km-value $1.4 \pm 0.1$ $\mathrm{mM}$ ) (Maier et al., 2002). Initially MstH kinetics were determined using the same range of substrate concentrations as for MstG. The results obtained suggested that MstH had a much higher affinity for glucose than MstG. Therefore the assay was optimised and repeated using a micro molar range of glucose concentrations. $\mathrm{MstH}$ was confirmed to transport glucose with apparent $\mathrm{Km}$-values of $0.06 \pm$ $0.005 \mathrm{mM}$ and a Vmax of $1.3 \pm 0.2 \mathrm{nmol} / \mathrm{min} / \mathrm{mg}$ DW (Figure 5.6), similar to what has been reported for $A$. niger MstA. 

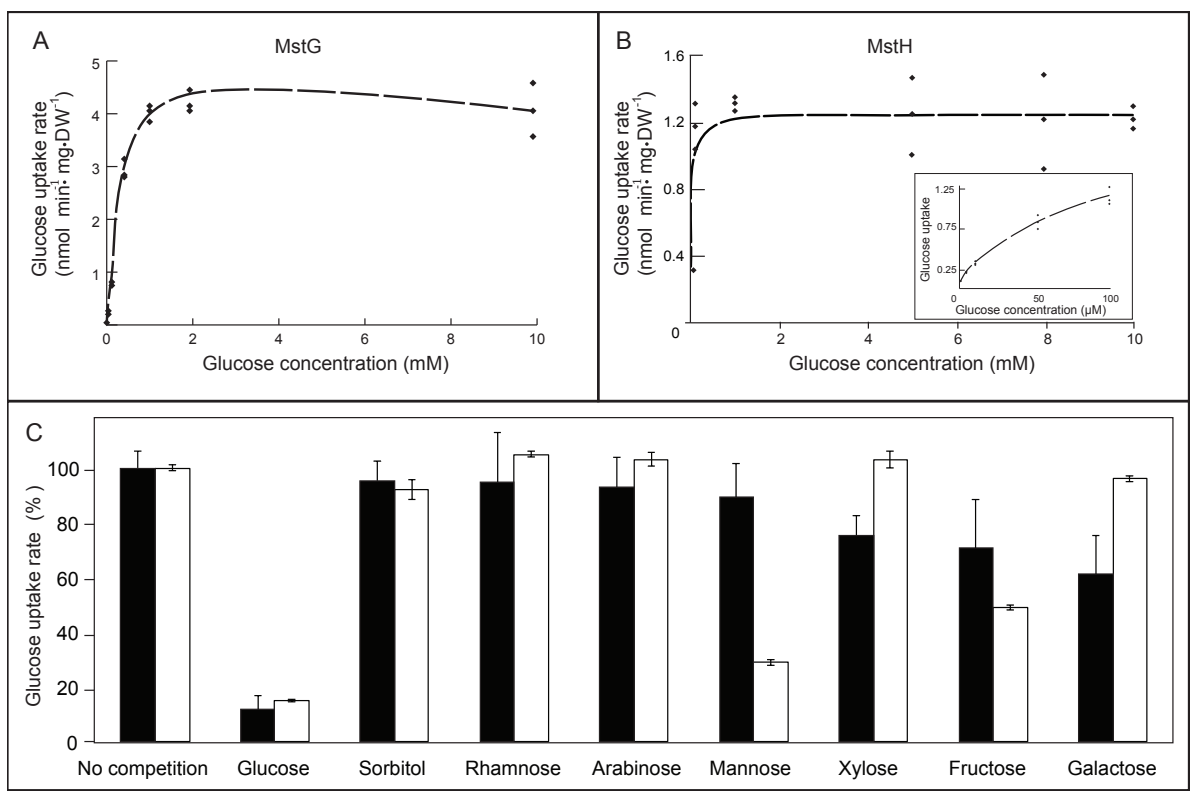

FIGURE 5.6. Uptake of ${ }^{14} \mathrm{C}$-labeled glucose by the yeast hexose transporter null mutant EBY.VW4000 expressing mstG or mstH as a function of glucose concentration. A and B: Diamonds represent measured uptake rates of ${ }^{14} \mathrm{C}$ labelled glucose $(\mathrm{n}=3)$ as a result of expression of $m s t G(\mathrm{~A})$ or $m s t H(\mathrm{~B})$. Black lines represent least-squares-fitted Michaelis-Menten model $\left(m s t G ; \mathrm{K}_{\mathrm{m}}=\right.$ $0.5 \mathrm{mM} ; \mathrm{Vmax}=5.8 \mathrm{nmol} / \mathrm{min} / \mathrm{mg} \mathrm{DW} ; m s t H: \mathrm{K}_{\mathrm{m}}=0.06 \mathrm{mM}$, and a $\mathrm{Vmax}=1.3 \mathrm{nmol} / \mathrm{min} / \mathrm{mg} \mathrm{DW})$. Insert: results for $m s t H$ using micro molar substrate concentrations. C: Uptake rate of $1 \mathrm{mM}{ }^{14} \mathrm{C}$-labelled glucose by EBY.VW4000 expressing $m s t G$ (Filled boxes) or $m s t H$ (Open boxes) in the presence of a 10-fold excess of competing sugars.

Since MstG and MstH were able to restore growth of the hexose non-transporting EBY.VW4000 on other monosaccharides as well, their substrate specificity was evaluated by determining glucose relative transport levels in the presence of a 10-fold excess of various competing sugars (Figure 5.6). Both transporters clearly showed higher specificities for glucose. However, besides glucose, galactose, fructose and xylose were able to inhibit MstG glucose transport by 39, 29 and $25 \%$ respectively, whereas in the $\mathrm{MstH}$ transformant mannose and fructose reduced the uptake of labelled glucose by 70 and $50 \%$, respectively. A. niger MstA also showed a preference for glucose, while being able to transport other 
monosaccharides as well (Vankuyk PA et al., 2004). Similarly, substrate specificity of Aspergillus nidulans hexose transporters $\mathrm{HxtB}$ and $\mathrm{HxtC}$ has been assayed (dos Reis et al., 2013), and a reduction of glucose uptake of 70 to $80 \%$ was observed for galactose, fructose and mannose. In accordance to the functioning of many glucose transporters from other filamentous fungi, the function of MstG and MstH was found to be energy dependent via proton symport. This was confirmed by measurements of glucose initial uptake rate in the presence of CCCP that resulted in a $60 \pm 8 \%(n=3)$ and $64 \pm 6 \%(n=3)$ reduction of labelled glucose uptake by $\mathrm{MstG}$ and $\mathrm{MstH}$ respectively.

\subsection{Conclusions}

In this study, two complementary approaches were used to both increase the understanding of the A. niger membrane-associated proteome, and to identify novel glucose transporters. In a purely in silico approach, a hidden Markov model ( $\left.\mathrm{HMM}_{\mathrm{gluT}}\right)$ was constructed for the identification of glucose transporters. $\mathrm{HMM}_{\text {gluT }}$ performed well in the identification and segmentation of functionally validated glucose transporters. In a complementary in vivo approach, defined culture conditions were applied to study the response of the A. niger membraneassociated proteome to different glucose concentrations. To the best of our knowledge, this is the first study on the membrane-associated proteome of the industrially relevant fungus $A$. niger. The study provided a better understanding of the membrane composition and topology, especially with respect to proteins with a putative transporter function; the A. niger transportome. Analysis of the A. niger in vivo transportome with $\mathrm{HMM}_{\text {gluT }}$ was shown to be effective; by combining the abundance patterns of the proteins identified in the experimental conditions with their respective $\mathrm{HMM}_{\text {gluT }}$ scores, two new putative glucose transporters, MstG and MstH, were identified. They were functionally validated in an engineered yeast strain with a monosaccharide transporter null-background and confirmed to be high affinity transporters for glucose.

\subsection{Methods}

\subsubsection{In silico transportome analysis and construction of a hidden Markov model specific for glucose transporters}

The A. niger ATCC1015 and S. cerevisiae CEN.PK proteomes plus their annotations, which were used for the in silico analysis, were obtained from the JGI 
database (Nordberg et al., 2013). For A. niger ATCC1015, only the best model proteome was used. Transmembrane helix domains were predicted with a standalone version of the TMHMM tool from the TMHMM website (Krogh et al., 2001). Hidden Markov models for the major facilitator superfamily ( $\mathrm{HMM}_{\mathrm{MFS}}$ ) and sugar porter $\left(\mathrm{HMM}_{\mathrm{SP}}\right)$ domains were obtained from the Pfam database (Punta et al., 2011).

The protein sequences used to build the $\mathrm{HMM}_{\text {gluT }}$ were obtained by entering the search term "sugar transporter" in the uniprot database and downloading the resultant $\mathrm{xml}$ and canonical fasta files. The $\mathrm{xml}$ file was parsed for proteins that were not experimentally verified to have the biological function assigned to them. The remaining proteins were divided into two separate datasets; the first dataset contained only the proteins with an experimentally verified GO term related to glucose transport (GO:0005355 and GO:0015758); the second dataset contained the remaining proteins (core dataset). The protein sequences of the experimentally verified glucose transporters were aligned by accessing the PRALINE structural alignment tool (Simossis and Heringa, 2005) via the SOAP interface and using the following settings: BLOSUM62, PSI-BLAST pre-profile processing (Homology extended alignment, 3 iterations), structural features: DSSP-defined secondary structure search, PSIPRED and TMHMM, fasta outputfile. The aligned fasta output was converted to the Stockholm format, and the $\mathrm{HMM}_{\text {gluT }}$ was built from the resultant output.sto file using the "hmmbuild" command from HMMER v3.0 (Finn et al., 2011). A. niger MstA was excluded from the multiple alignment.

For the 3 -fold cross validation, the initial dataset, containing the 42 verified glucose transporters, was randomly partitioned into three equally sized subsets. The model was then built from every combination of 2 subsets, while the validation of the model was carried out on the core dataset plus the third subset of the glucose transporters not used for the model building process. This allowed for the determination of false and true negatives in comparison to false and true positives, and thus gave an estimate of the prediction power of the model. The 3 -fold cross validation was repeated 10 times, each time with different random subsets of the verified glucose transporters. Two thresholds were determined from the average ROC curve resulting from the $10 \times 3$-fold cross validation of the HMM $_{\text {gluT: }}$

- Minimal distance to the point $\left[\begin{array}{ll}0 & 1\end{array}\right]\left(\mathrm{d}_{\min }\right)$ :

With $\mathrm{sn}=$ sensitivity $(\mathrm{TPR})$ and $\mathrm{sp}=$ specificity $(\mathrm{TNR})$. 
Another metric to calculate the optimal trade-off point between true and false predictions is the Matthews correlation coefficient. The Matthews correlation coefficient is essentially a one number representation of the confusion matrix, and is thus suitable to compare the outcome of different model predictions. This coefficient is calculated as follows:

- Matthews correlation coefficient (MCC):

Here, a value closer to 1 is clearly a better prediction, and thus the inferred $\mathrm{HMM}_{\text {gluT }}$ score at the highest MCC value calculated along the ROC curve, $\mathrm{MCC}_{\max }$, was set as second threshold.

Similarly, the Blast approach was evaluated by using each of the 42 verified glucose transporters as separate query for a local Blastp search against the core dataset plus the other verified glucose transporters, allowing for the determination of true and false positive rates (see additional file 2).

\subsubsection{Strains and growth conditions}

Escherichia coli DH5 $\alpha$ (endA1, hsdR17, gyrA96, thi-1, relA1, supE44, recA1, $\Delta$ lacU169 (Ф 80 lacZ $\Delta \mathrm{M} 15)$ ), grown at $37^{\circ} \mathrm{C}$, was used for cloning experiments and plasmid propagation. Luria Broth (LB) was used as growth medium (1\% $\mathrm{w} / \mathrm{v}$ tryptone, $0.5 \% \mathrm{w} / \mathrm{v}$ yeast extract, $1 \% \mathrm{w} / \mathrm{v} \mathrm{NaCl}$ ) with or without $100 \mu \mathrm{g} / \mathrm{mL}$ ampicillin.

The wild type strain $A$. niger N400 (CBS 120.49), used for the plasma membrane proteomics analysis, was grown at $30{ }^{\circ} \mathrm{C}$ on complete medium plates for spores generation and maintenance (Pontecorvo et al., 1953). Mycelial biomass of the N400 strain was obtained after $18 \mathrm{~h}$ of growth in liquid cultures containing minimal medium (Pontecorvo et al., 1953), including $5 \mathrm{~g} / \mathrm{L}$ of yeast extract, with $100 \mathrm{mM}$ sorbitol as carbon source. Equal amounts of water-rinsed mycelium were transferred to 1-liter benchtop fermenters (Sartorius) with $750 \mathrm{~mL}$ of minimal medium containing $4.50 \mathrm{~g} \mathrm{NaNO}_{3}, 1.13 \mathrm{~g} \mathrm{KH}_{2} \mathrm{PO}_{4}, 0.38 \mathrm{~g} \mathrm{KCl}, 0.38 \mathrm{~g} \mathrm{MgSO}_{4} \cdot 7$ $\mathrm{H} 2 \mathrm{O}$ and $750 \mu \mathrm{L}$ of Vishniac solution (Pontecorvo et al., 1953; Vishniac and Santer, 1957). Three different conditions, varying on the carbon source composition, were studied: sorbitol $100 \mathrm{mM}$ (reference condition), sorbitol $100 \mathrm{mM}$ plus glucose $1 \mathrm{mM}$ (low-glucose condition) and sorbitol $100 \mathrm{mM}$ plus glucose $60 \mathrm{mM}$ (high-glucose condition). Two biological replicates per condition were studied. Fermenters were stirred at 1'000 rpm and aerated with filtered air $(0.6 \mathrm{~L} / \mathrm{min})$, keeping oxygen levels over $60 \%$. The initial $\mathrm{pH}$, set at 4.0 , was allowed to drop until pH 3.5 and kept constant afterwards by sodium hydroxide addition. 
Mycelium samples for RT-qPCR analysis were obtained from a mycelium transfer experiment similar to the one described above, using minimal medium with the following carbon source compositions: $100 \mathrm{mM}$ sorbitol (reference condition), glucose $5 \mathrm{mM}$, glucose $55 \mathrm{mM}$, fructose $5 \mathrm{mM}$, fructose $55 \mathrm{mM}$, mannose 5 $\mathrm{mM}$ and mannose $55 \mathrm{mM}$. Two biological replicates per condition were studied as well. Cultures were performed in erlemeyer flasks, stirred at $200 \mathrm{rpm}$ and the initial $\mathrm{pH}$ of the medium in all conditions was set at 4.0. Two hours after inoculation mycelium samples were taken and quickly washed, dried with a single-use towel, snap-frozen with liquid nitrogen and stored at $-80{ }^{\circ} \mathrm{C}$ until further processing.

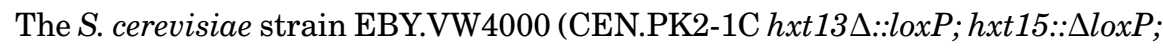

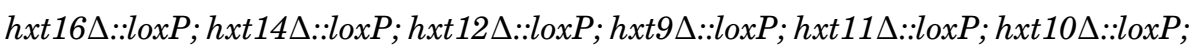

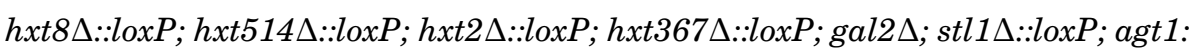
:loxP; ydl247w $\Delta:: l o x P ; y j r 160 c \Delta:: l o x P$ ), used for the functional complementation experiments and the characterisation of glucose transporters (Wieczorke et al., 1999), was grown at $30^{\circ} \mathrm{C}$ and maintained in solid complete medium containing $10 \mathrm{~g} / \mathrm{L}$ of yeast extract, $20 \mathrm{~g} / \mathrm{L}$ of peptone and $20 \mathrm{~g} / \mathrm{L}$ of maltose. The EBY.VW4000 derived strains obtained in the present study where grown in liquid minimal medium containing $6.7 \mathrm{~g} / \mathrm{L}$ of yeast nitrogen base with ammonium sulphate (Difco), $20 \mathrm{~g} / \mathrm{L}$ of maltose, supplemented with leucine (30 mg/L), tryptophan (20 $\mathrm{mg} / \mathrm{L})$ and histidine $(20 \mathrm{mg} / \mathrm{L})$.

\subsubsection{A. niger membrane associated protein purification and quality control analysis}

A. niger mycelium samples (2-3 g, press-dried), washed with a $20 \mathrm{mM}$ HEPES buffer $\mathrm{pH} 7.6$ containing $150 \mathrm{mM} \mathrm{NaCl}$, and resuspended in the same solution containing $1 \%(\mathrm{v} / \mathrm{v})$ protease inhibitor cocktail for yeast and fungi (Sigma), were mechanically disrupted using a French press (8'000 psi). Cell free extracts were centrifuged for $5 \mathrm{~min}$ at low speed (500 g), in order to remove unbroken cells and pellet debris. The supernatants were then centrifuged during $20 \mathrm{~min}$ at medium speed (5’000 g), to pellet and remove remaining heavy organelles. The remaining supernatants were centrifuged for $120 \mathrm{~min}$ at high speed ( $\left.\sim 85^{\prime} 000 \mathrm{~g}\right)$, to pellet light organelles (P3).

P3 pellets were resuspended using a Dounce homogenizer in $1 \mathrm{~mL}$ of a 20 $\mathrm{mM}$ HEPES buffer $\mathrm{pH} 7.6$ containing $250 \mathrm{mM}$ sucrose. P3 suspensions were overlayed in a discontinuous sucrose density gradient, prepared by layering successive decreasing sucrose densities solutions $(6 \times 1 \mathrm{~mL})$, with concentrations 
ranging from $1.20 \mathrm{M}$ to $0.70 \mathrm{M}$, upon one another. Sucrose density gradients were centrifuged ( $\sim 100$ ’000 g - $60 \mathrm{~min}$ ) to isolate different membrane-associated fractions from P3 pellet. Five fractions are obtained (P3A, P3B, P3C, P3D and P3E).

\subsubsection{Sample preparation for LC-MS/MS}

The protein content of enriched plasma membrane associated fractions was determined using the BCA protein assay. Membrane proteins were solubilised by mixing volumes of each fraction containing $25 \mu \mathrm{g}$ of protein with equal volumes of a 2 x solution of $20 \mathrm{mM}$ HEPES $\mathrm{pH} 7.6$ containing $1 \mathrm{M}$ 6-aminocaproic acid and $10 \mathrm{~g} / \mathrm{L}$ of $\mathrm{n}$-dodecyl-beta-D-maltoside. Cell membrane-detergent mixes were incubated in a thermoblock (ThermoMixer) for $1 \mathrm{~h}$ at $20{ }^{\circ} \mathrm{C}$ and vigorous stirring (1'000 rpm). Afterwards, samples were sonicated in a water bath for $15 \mathrm{~min}$, and finally they were centrifuged at 22 ' $000 \mathrm{~g}$ for $30 \mathrm{~min}$. Supernatants containing solubilised proteins were concentrated using MMicrocon YM-10 columns (cutoff, 10 kDa; Millipore, Eschborn, Germany) and loaded into a $12 \%$ SDS-polyacrylamide gel, which was run until the loaded samples entered into the gel. The gel was stained according to the manufacturer's instructions using Page Blue staining (Fermentas) and rinsed with ultrapure water. Each sample-gel lane was cut into one slice (approx. $1 \mathrm{~cm}^{2}$ ), carefully sliced into smaller pieces of about 1 $\mathrm{mm}^{3}$ and transferred into microcentrifuge tubes. Samples were destained and equilibrated through three washing steps using the following solutions: $50 \mathrm{mM}$ ammonium bicarbonate (ABC) (incubated $5 \mathrm{~min}$ ), ABC/acetonitrile (1:1, v/v) (incubated $5 \mathrm{~min}$ ) and neat acetonitrile (incubated $5 \mathrm{~min}$ ). These washing steps were successively repeated two times. The gel samples were then swelled in 10 $\mathrm{mM}$ dithiothreitol (DTT) for $20 \mathrm{~min}$ at $56{ }^{\circ} \mathrm{C}$ to reduce protein disulfide bonds. Subsequently, the DTT solutions were removed and samples were alkylated with $50 \mathrm{mM}$ 2-chloroacetamide in $\mathrm{ABC}$, for $20 \mathrm{~min}$, at room temperature, in the dark. The 2-chloroacetamide solutions were removed, and samples were again washed twice with: neat acetonitrile (incubated 5 times), ABC (incubated $5 \mathrm{~min}$ ) and neat acetonitrile (incubated 5 times). Approximately $150 \mu \mathrm{L}$ of digestion buffer, containing sequencing grade modified trypsin $(12.5 \mathrm{ng} / \mu \mathrm{L}$ ) (Promega, Madison, WI) in ABC, was added to each sample, making sure that all gel pieces were kept wet during digestion (adding, if necessary, additional ABC solution). Protein samples were digested overnight at $37^{\circ} \mathrm{C}$. Peptide digestion products were extracted by adding $50 \mu \mathrm{L}$ of $2 \%$ trifluoroacetic acid (TFA), followed by an incubation step in a thermoblock (ThermoMixer) for $20 \mathrm{~min}$, at room temperature and vigorous 
stirring (1400 rpm). Gel pieces were then subjected to $20 \mathrm{~s}$ sonication in a water bath, centrifuged and supernatants were transferred to new tubes. The peptide extraction step was then repeated once by washing the gel pieces with buffer $\mathrm{B}$ ( $80 \%$ acetonitrile, $0.1 \%$ formic acid) followed by the mentioned incubation and sonication steps. Supernatants from both extractions were pooled and samples were placed in a vacuum centrifuge for acetonitrile evaporation util 20-40 $\mu \mathrm{L}$ were left. Finally, samples were acidified by addition of TFA (1:1, v/v) and peptide clean-up procedure, prior to LC-MS/MS analysis, was performed using the "STop And Go Extraction" prodedure as described before (Rappsilber et al., 2003).

\subsubsection{Mass spectrometric measurements}

LC-MS/MS analysis was performed at Radboud Proteomics Centre as described previously (Rajala et al., 2015). Measurements were performed by nanoflow reversed-phase C18 liquid chromatography (EASY nLC, Thermo Scientific) coupled online to a 7 Tesla linear ion trap Fourier-Transform ion cyclotron resonance mass spectrometer (LTQ FT Ultra, Thermo Scientific).

\subsubsection{Proteomics data analysis}

The LC-MS/MS spectra obtained from the proteomics experiment were identified and quantified using the maxQuant software (Cox and Mann, 2008). The peptides were mapped against the annotated $A$. niger ATCC1015 in silico proteome obtained from the JGI database (http://genome.jgi-psf.org/Aspni7/Aspni7.home.html) using the default settings of the maxQuant version 1.3.0.5 (variable modifications: oxidation (M) and acetylation (protein N-term); enzyme used: trypsin/P; fixed modifications carbamidomethyl (cys)), except for the variables affecting the label free quantification. For this, the multiplicity was set to 1, and the parameters for label-free quantification as well as the $\mathrm{iBAQ}$ and peak property calculations were selected. Only proteins with 2 or more unique peptide hits were considered for further analysis. Protein localisation was determined using the softberry protComp prediction server (http://linux1.softberry.com). The relative abundances of the identified proteins are represented as follows:

$\mathrm{AL}=$ relative abundance of the protein in the low-glucose condition

$\mathrm{AH}=$ relative abundance of the protein in the high-glucose condition

$\mathrm{AS}=$ relative abundance of the protein in the reference (sorbitol) condition 


\subsubsection{Transcriptional analysis of $m s t G$ and $m s t H$ genes}

Mycelium samples were disrupted with glass beads in a Fastprep-24 instrument, and RNA extraction was performed by a Maxwell 16 instrument using the Maxwell 16 LEV simplyRNA kit (Promega). Reverse transcription and qPCR analysis were performed following the protocols and instruments described in MachAigner et al. (2012). The previously described histone-like gene "hist" transcript (gene ID 207921) was used as reference for normalisation of the expression data (Mach-Aigner et al., 2012). The following sequences belong to the primers used for qPCR analysis in this study: hist-FW: ACAATGACTGGCCGTGGAAAGG, histRV: ATACGCTTGACACCACCACGAC, mstG-FW: CGGTGGTGGTATGGCTTTCT, mstG-RV: GTTCTCAGGCACACCGTACA, mstH-FW: GCCATCATGATCGGTCTGTTTGTC, mstH-RV: ACTGATGGTTCCGGTGTCATATCC.

\subsubsection{Construction of S. cerevisiae EBY.WV4000 transformants expressing $A$. niger $m s t G$ and $m s t H$ genes and glucose uptake assays}

The coding sequence of the genes $m s t G$ and $m s t H$, digested with SpeI and XhoI were cloned on the $S$. cerevisiae expression vector p426HXT7-6His, previously linearised with SpeI and XhoI under the control of the constitutive promoter HXT7p and the terminator CYC1t. Transformation of S. cerevisiae EBY.WV4000 with p426HXT7-6His (empty vector), p426HXT7-6His_mstG and p426HXT76His_mstH was performed as described (Gietz and Woods, 2002).

Uptake assays were performed as described with minor adjustments (Walsh et al., 1994). $5 \mathrm{~mL}$ of Synthetic Complete medium without uracil (SC-ura; 6.7\% YNB Difco + dropout supplement mix without uracil Sigma-Aldrich) with $2 \%$ maltose as a carbon source was inoculated from plate with EBY.VW4000 with pRS426H7 (control) and EBY.VW4000 with pRS426H7_mstG or pRS426H7_mstH and incubated overnight $\left(30^{\circ} \mathrm{C}, 225 \mathrm{rpm}\right)$ as pre-inoculum. Pre-inoculum was transferred to $500 \mathrm{~mL}$ SC-ura with $2 \%$ maltose and incubated for $24 \mathrm{~h}$. Cells were harvested by centrifugation (4'000 g, $10 \mathrm{~min}$ ) and washed with $50 \mathrm{~mL}$ ice-cold MQ. Cells were then resuspended in $2 \mathrm{~mL}$ ice-cold SC-ura without carbon source, divided in $40 \mu \mathrm{L}$ aliquots and kept on ice.

Aliquots were incubated for $5 \mathrm{~min}$ at $30{ }^{\circ} \mathrm{C}$ before uptake assay was started. To start the reaction $10 \mu \mathrm{L}$ of a 5 times concentrated labelled glucose solution (D[U-14C]-glucose, Perkin Elmer) was added. After exactly 10 seconds the reaction was stopped by the addition of $1 \mathrm{~mL}$ of $100 \mathrm{mM} \mathrm{LiCl}$ and vacuum filtration $(0.45$ $\mu \mathrm{m}$ HV filters, 1225 sampling manifold, Millipore), with subsequent washing with 
$2 \times 5 \mathrm{~mL}$ of ice-cold $100 \mathrm{mM} \mathrm{LiCl}$. After 5 min of drying in the vacuum manifold, the filters were transferred to scintillation vials with $7.5 \mathrm{~mL}$ scintillation liquid (Ultima Gold, Perkin Elmer) and activity was counted (Packard Tricarb 1600TR). All reactions were performed in triplicates. For each reaction, a negative control assay without incubation was performed.

To determine transport kinetics, uptake of labelled glucose with a range of concentrations from $1 \mu \mathrm{M}$ to $10 \mathrm{mM}$ was measured in SC-ura at $\mathrm{pH}$ 5.0. Glucose solutions with an activity of approximately 700 to 70 '000 Bq were used. To determine kinetic parameters $\mathrm{Km}$ and Vmax, the data was fitted to the following Michaelis-Menten model with substrate inhibition using the least squares method.

To determine substrate specificity, the uptake of $1 \mathrm{mM}$ of labelled glucose was measured in the presence of $10 \mathrm{mM}$ of a competing carbon source. D-glucose, D-sorbitol, D-xylose, D-mannose, L-rhamnose, L-arabinose, D-fructose and Dgalactose were selected as competing carbon sources.

To determine if the transporter functions via proton symport, activity was measured while uncoupling the proton gradient by carbonyl cyanide m-chlorophenyl hydrazine (CCCP). This inhibitor is dissolved in DMSO and added before temperature equilibration of the cells. The uptake rate in the presence of $250 \mu \mathrm{M}$ of CCCP and $2 \%$ DMSO was compared with the uptake rate in the presence of only $2 \%$ DMSO.

\subsection{Acknowledgements}

This work has been carried out on the basis of a grant in the framework of the BEBASIC program F01.011 Transport processes in the production of organic acids by Aspergillus niger. We would like to thank Dr Eckhard Boles for providing us the $S$. cerevisiae strain EBY.VW4000 and the plasmid p426HXT7-6His and Adrie Westphal for his suggestions on the enzyme kinetics.

\section{Supplementary information}

Supplementary files for this chapter can be found online at:

https://biotechnologyforbiofuels.biomedcentral.com/articles/10.1186/s13068-0150317-9\#MOESM1 



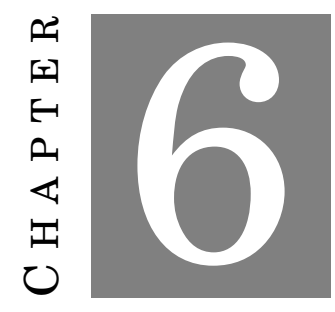

IDENTIFICATION AND FUNCTIONAL CHARACTERIZATION OF NOVEL XYLOSE TRANSPORTERS FROM THE CELL FACTORIES Aspergillus niger AND Trichoderma reesei

This chapter is adapted from:

Sloothaak J*, Tamayo-Ramos JA*, Odoni DI, Laothanachareon T, Derntl C, Mach-Aigner A, Martins dos Santos VAP, Schaap PJ. (2016). Identification and functional characterization of novel xylose transporters from the cell factories Aspergillus niger and Trichoderma reesei. In Biotechnology for biofuels (https://biotechnologyforbiofuels.biomedcentral.com/articles/10.1186/s13068016-0564-4).

*equal contributions 


\subsection{Abstract}

Background: Global climate change and fossil fuels limitations have boosted the demand for robust and efficient microbial factories for the manufacturing of bio-based products from renewable feedstocks. In this regard, efforts have been done to enhance the enzyme-secreting ability of lignocellulose-degrading fungi, aiming to improve protein yields while taking advantage of their ability to use lignocellulosic feedstocks. Access to sugars in complex polysaccharides depends not only on their release by specific hydrolytic enzymes, but also on the presence of transporters capable of effectively transporting the constituent sugars into the cell. This study aims to identify and characterize xylose transporters from Aspergillus niger and Trichoderma reesei, two fungi that have been industrially exploited for decades for the production of lignocellulose-degrading hydrolytic enzymes.

Results: A hidden Markov model for the identification of xylose transporters was developed and used to analyse the $A$. niger and $T$. reesei in silico proteomes, yielding a list of candidate xylose transporters. From this list, three A. niger (XltA, XltB and XltC) and three T. reesei (Str1, Str2 and Str3) transporters were selected, functionally validated and biochemically characterized through their expression in a $S$. cerevisiae hexose transport null mutant, engineered to be able to metabolize xylose but unable to transport this sugar. All six transporters were able to support growth of the engineered yeast on xylose but varied in affinities and efficiencies in the uptake of the pentose. Amino acid sequence analysis of the selected transporters showed the presence of specific residues and motifs recently associated to xylose transporters. Transcriptional analysis of $A$. niger and $T$. reesei showed that XltA and Str1 were specifically induced by xylose and dependent on the XlnR/Xyr1 regulators, signifying a biological role for these transporters in xylose utilization.

Conclusions: This study revealed the existence of a variety of xylose transporters in the cell factories A. niger and T. reesei. The particular substrate specificity and biochemical properties displayed by A. niger XltA and XltB suggested a possible biological role for these transporters in xylose uptake. New insights were also gained into the molecular mechanisms regulating the pentose utilization, at inducer uptake level, in these fungi. Analysis of the A. niger and T. reesei predicted transportome with the newly developed hidden Markov model showed to be an efficient approach for the identification of new xylose transporting proteins. 


\subsection{Background}

Industrial production of chemicals and enzymes synthesized by fungi comprises a huge international market (Jang et al., 2012; Magnuson and Lasure, 2004; Punt et al., 2002) and filamentous fungi such as Aspergillus niger and Trichoderma reesei have become two of the main workhorses of today's industrial biotechnology. Their high enzyme secretory capacity has already been industrially exploited during the last decades and both species have the capacity to efficiently degrade and utilize second-generation lignocellulosic feedstocks (van den Brink and de Vries, 2011). Global climate change, fossil fuels limitations and breakthroughs in the advanced biofuels market have boosted the demand for robust and efficient microbial cell factories. In this regard, to enhance the enzyme-secreting ability of these fungi, efforts at multiple levels have been done: from studies at systems level to understand the secretory process, to the use of more applied strategies focused on the improvement of protein yields (de Oliveira and de Graaff, 2011; Lubertozzi and Keasling, 2009). Nevertheless, access to sugars released from the complex polysaccharides is not only dependent on the ability of these fungi to secrete high titers of a complex mix of hydrolytic enzymes, but also on the presence of a large array of sugar porters, transport proteins that are capable of effectively transporting the constituent sugars into the cell.

Most of the current knowledge on sugar transporters in fungi comes from studies in the model organism Saccharomyces cerevisiae. S. cerevisiae is able to consume a limited set of mono- and disaccharides (Leandro et al., 2009). When the in silico proteome is explored with Pfam profile hidden Markov models (HMMs) (Punta et al., 2011) for different transporter proteins, 73 proteins may be classified as major facilitator superfamily (MFS, Pfam ID: PF07690) transporters, of which 43 belong to the sugar porter (SP, Pfam ID: PF00083) subfamily. The superior capacity of lignocellulose-degrading fungi for sugar uptake seems clear from the number of sugar porters that are available. In the A. niger in silico proteome 469 proteins may be classified as MFS transporters of which 256 are included in the SP subfamily (Sloothaak et al., 2015). Using the same approach we estimate that for $T$. reesei 235 proteins can be classified as MFS transporters of which 113 can be classified as SP.

In order to completely eliminate the ability of $S$. cerevisiae to use glucose as a carbon source $20 \mathrm{SP}$ genes needed to be knocked-out (Wieczorke et al., 1999). This apparent redundancy enables the organisms to efficiently take up the available carbon source in response to a wide variety of conditions. In yeast not only sugar transporters contribute to this flexibility, but also a number of sensors that share 
with these transporters the same domain architecture, but have an additional domain to connect to intracellular signal transduction pathways (Coons et al., 1997; Ozcan et al., 1996). While glucose is always the preferred substrate, many of these yeast transporters have the ability to transport several other sugars including natively non-fermentable substrates like xylose. Members of the sugar porter subfamily are structurally similar in design, with 12 transmembrane segments organized in two distinct domains (Madej and Kaback, 2013; Sun et al., 2012) and only a limited number of amino acid side chains are believed to control binding and affinity (Guan and Kaback, 2006; Quistgaard et al., 2013). In search for more efficient xylose transporters recent studies have focused on changing the functionality of known yeast transporters and it has been shown that it is possible to change the uptake rate, affinity and inhibition through a limited set of mutations (Farwick et al., 2014; Nijland et al., 2014; Reznicek et al., 2015; Young et al., 2012, 2014). Native industrially relevant xylose transporters that show high affinity and capacity for xylose uptake have been identified from Escherichia coli, Pichia stipitis and Candida intermedia (Leandro et al., 2006; Weierstall et al., 1999). Additionally several transporters from filamentous fungi have been reported to transport xylose, such as An25 and An29-2 from Neurospora crassa, XtrD from A. nidulans, MstA from A. niger and Xlt1 and Str1 from T. reesei (Colabardini et al., 2014; Du et al., 2010; Huang et al., 2015; Saloheimo et al., 2007). Of this list only An25, and MstA have been biochemically characterized.

A. niger and T. reesei are natural xylose metabolizers. The aim of this study was to study their modus operandi in xylose transport. This was done through the identification, characterization and regulation analysis of a novel set of native transporters able to use xylose as substrate. To select promising new candidates, a hidden Markov model specific for xylose transporters $\left(\mathrm{HMM}_{\mathrm{xylT}}\right)$ was developed, similarly to the HMM developed for glucose transporters (Sloothaak et al., 2015). With $\mathrm{HMM}_{\mathrm{xylT}}$ the in silico proteomes of $A$. niger and T. reesei were mined for candidate xylose transporters and from each species three candidate xylose transporters were selected for experimental validation and biochemical characterization. All six transporters were able to transport xylose and between them they showed remarkable differences in terms of transcriptional expression regulation and substrate affinity and selectivity. 


\subsection{Results and Discussion}

\subsubsection{A. niger and T. reesei in silico proteome mining}

Proteins belonging to the SP family are structurally similar, but share a low level of sequence identity (Sloothaak et al., 2015) and only a limited number of amino acid side chains are believed to be involved in binding and affinity (Guan and Kaback, 2006; Quistgaard et al., 2013). As discussed in our previous work, HMMs can be used to more effectively segregate SP proteins based on their substrate than standard BLAST-based methods (Sloothaak et al., 2015). However, precision will largely depend on the availability of a consistent training set of previously characterized proteins with the function of interest. Given the data available in literature, we aimed to build a profile HMM as specific for xylose transporters as possible. To this end, we retrieved protein sequences from 24 functionally validated transporters that are able to transport xylose, with the additional requirement that they must originate from species that are naturally able to metabolize xylose (Additional file 1). Note that, when biochemically characterized, most of these xylose transporters either performed (at least) equally well on glucose, or were inhibited by the hexose, and thus the list is far from ideal. Nonetheless, we expected that residues fundamental for xylose transport are conserved in these proteins, ultimately making them xylose transporters as well. The $\mathrm{HMM}_{\mathrm{xylT}}$ built from these sequences was used to mine the in silico proteomes of $A$. niger and $T$. reesei for putative xylose transporters. The complete workflow applied for the selection of candidate xylose transporters is presented in Figure 6.1, additional files 2 and 3 provide details on $\mathrm{HMM}_{\mathrm{xylT}}$. The output list of top scoring $A$. niger and T. reesei proteins was analyzed (Additional file 4), using the available literature, to search for the most promising candidates for xylose utilization. In this regard, the A. niger $\mathrm{HMM}_{\mathrm{xylT}}$ output was compared with different transcriptome analysis studies where $A$. niger was grown in the presence of xylose, xylose-arabinose mixes, sugarcane bagasse, straw or willow (de Souza et al., 2011, 2013; Delmas et al., 2012; Pullan et al., 2014; van der Veen et al., 2009). The combined results of the $\mathrm{HMM}_{\mathrm{xylT}}$ output and transcriptome analyses indicated that many of the new $A$. niger candidate transporters with a high $\mathrm{HMM}_{\mathrm{xylT}}$ score were transcriptionally upregulated in, at least, one of these culture conditions (Additional file 5). Moreover, many of them were also possibly regulated by the XlnR and AraR transcription factors, which control hemicellulose utilization in A. niger (de Souza et al., 2013). This suggested that the top scoring sugar porters within the A. niger $\mathrm{HMM}_{\mathrm{xylT}}$ list contained a number of good candidates for further analysis. In the case of the $T$. 
reesei top scoring sugar porters, only a few transcriptomic studies highlighting the expression modulation of hypothetical sugar transporters in hemicellulose degradation conditions are available. Still, in two recently published studies, analyzing the $T$. reesei transcriptomic response to wheat straw, many of the $\mathrm{HMM}_{\mathrm{xylT}}$ top scoring transporters were upregulated on this carbon source versus glucose (Bischof et al., 2013; Ries et al., 2013). We also found that the function of three of the top scoring transporters had been studied previously (Huang et al., 2015; Ramos et al., 2006; Saloheimo et al., 2007; Zhang et al., 2013). One of them, TrStr1 (protID 50894) (Huang et al., 2015) was confirmed to function as xylose transporter, while Stp1 (protID 47710) and TrHxt1 (protID 22912) were associated to cellobiose and glucose utilization respectively (Ramos et al., 2006; Zhang et al., 2013).

\subsubsection{Phylogenetic clustering of top scoring $A$. niger and $T$. reesei transporters}

With the help of $\mathrm{HMM}_{\mathrm{xylT}}$, a number of candidate xylose transporters from A. niger and T. reesei could be identified. Further analysis of the $\mathrm{HMM}_{\mathrm{xylT}}$ output showed that the possible function of many high scoring transporters could be linked to hemicellulose-associated sugars, particularly in A. niger. For this reason, three top 10 A. niger transporters AnXltA (protID 1169204), AnXltB (protID 1127588) and AnXltC (protID 1167504) were selected for further studies. For the selection of $T$. reesei xylose transporter candidates the top 15 highest scoring proteins from $A$. niger and $T$. reesei were aligned and a neighbor-joining distance tree was built from the pairwise distances (Figure 6.1). Broadly five protein clusters were observed. AnXltA and AnXltB are in cluster 1 and within this cluster A. niger XltA showed a strong clustering with T. reesei Str1 (protID 50894). Str1 was recently confirmed to be essential for pentose utilization and relevant in the induction of the hemicellulose utilization system (Huang et al., 2015), and since this transporter was not yet biochemically characterized it was selected for further study. In the same cluster, T. reesei protein 121482 (TrStr2) appeared to be a close homolog of $A$. niger XltB and thus TrStr2 was also selected for further analysis.

Several transporters from cluster 4 were previously studied, showing the relevance of this subgroup for sugar uptake (Sloothaak et al., 2015; Vankuyk PA et al., 2004; Zhang et al., 2013). Taking this into account, and the fact that MstA has been shown to have high affinity towards xylose, we decided to select another T. reesei representative of this cluster (TrStr3, ProtID 62380) for further studies. 

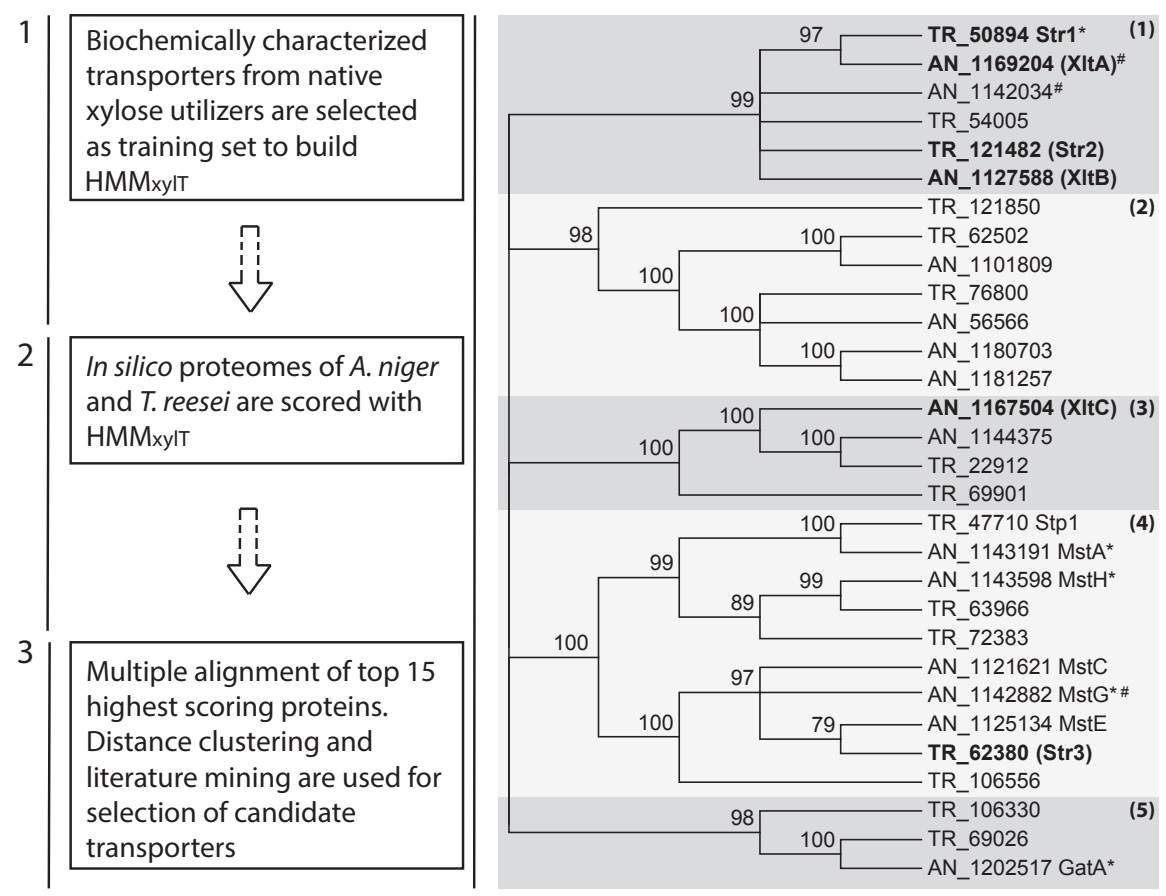

FIGURE 6.1. Approach to candidate transporters selection and neighbor joining distance tree of top 15 highest HMMxyl scoring proteins from $A$. niger and $T$. reesei. Functionally validated xylose transporters were retrieved to train a $\mathrm{HMM}_{\mathrm{xylT}}$, used to analyze the in silico proteomes of $A$. niger and $T$. reesei. An analysis of the phylogenetic relationships of the top scoring candidate transporters, taking into account the available literature, allowed the selection of candidate transporters that were subsequently characterized. Bootstrap values are indicated, nodes with bootstrap values $<75 \%$ are collapsed. Numbers indicate protein ID in JGI online genomes of A. niger ATCC1015 and T. reesei v2.0 (Koike et al., 2013; Martinez et al., 2008). Proteins selected for further biochemical characterization are indicated in bold with protein names in brackets. Abbreviations; $\mathrm{AN}_{-}$, A. niger; $\mathrm{TR}_{-}$, T. reesei; * indicate previously experimentally validated transporters; \# indicate the presence of a XlnR-binding motif as suggested by Van Peij et al. (1998) in the 1 $\mathrm{kb}$ upstream region of the encoding gene.

\subsubsection{Engineering of a laboratory-evolved yeast strain for functional validation of xylose transporters}

S. cerevisiae EBY.VW4000 is a strain unable to transport glucose and is frequently used for functional validation of sugar transporters (Wieczorke et al., 1999). For 
the purpose of ex vivo functional validation of the selected fungal transporters strain EBY.VW4000 was genetically modified to be able to metabolize xylose as a carbon source and laboratory-evolved for an enhanced growth on xylose. For this, strain EBY.VW4000, unable to grow on glucose, mannose, galactose or fructose as carbon source, was transformed with the plasmid pRH315, expressing the $P$. stipitis D-xylose reductase (XYL1) and xylitol dehydrogenase (XYL2) genes, and the $S$. cerevisiae xylulokinase (XKS1) gene (Hector et al., 2011). An isolated transformant of the EBY.VW.4000 strain expressing the xylose utilization pathway (EBY.XP) was subsequently transformed with a plasmid expressing the $A$. niger hypothetical xylose transporter XltA (p426HXT7-6His-xltA). EBY.XP $x l t A$ transformants were isolated and the ability of XltA to confer them the capacity to grow in the presence of xylose was studied. Ten-fold serial dilutions of exponentially growing cells from an $x l t A$ transformant were spotted on different minimal medium plates supplemented with either $1 \%(\mathrm{w} / \mathrm{v})$ maltose or $1 \%$ $(\mathrm{w} / \mathrm{v})$ xylose. After an incubation period of 5 days, the $x l t A$ transformant strain showed ability to grow on xylose (Figure 6.2). While this result confirmed that $A$. niger XltA is able to transport xylose, the ability of the transformant strain to grow on the pentose was poor. Poor growth of xylose utilizing yeast transformant strains has been described before (Colabardini et al., 2014; Huang et al., 2015; Saloheimo et al., 2007), and this could be due to metabolic imbalances as a result of different cofactor specificities of the heterologous genes XYL1 and XYL2 present in the modified $S$. cerevisiae strain (Hector et al., 2011). To obtain a better growing xylose-utilizing host for routine identification of xylose transporters, the ability of the EBY.XP strain to metabolize xylose was improved through laboratory-evolution. For this, the strain was grown in agar plates containing selective minimal medium with $2 \%(\mathrm{w} / \mathrm{v})$ xylose, during long-term incubations (at least two weeks), at $30{ }^{\circ} \mathrm{C}$. Once growing colonies were observed, they were pooled and re-plated again in $2 \%(\mathrm{w} / \mathrm{v})$ xylose plates. After two re-plating rounds, several single colonies showing relative fast growth were selected and re-plated individually. Finally, a thus evolved strain showing the fastest growth, called $\mathrm{Ag} 11$, was selected for further research. Prior to further use as an optimized host for identification of new xylose transporters through functional complementation of transport, the expression vector carrying the $A$. niger $x l t A$ gene was cured from the Ag11 strain (see Methods for details). The cured strain Ag11C3 was confirmed by its inability to grow in the absence of uridine, and in the presence of xylose as single carbon source.

To confirm that laboratory-evolved strain Ag11C3 could be successfully used as a host for the identification of new xylose transporters, the strain was re- 


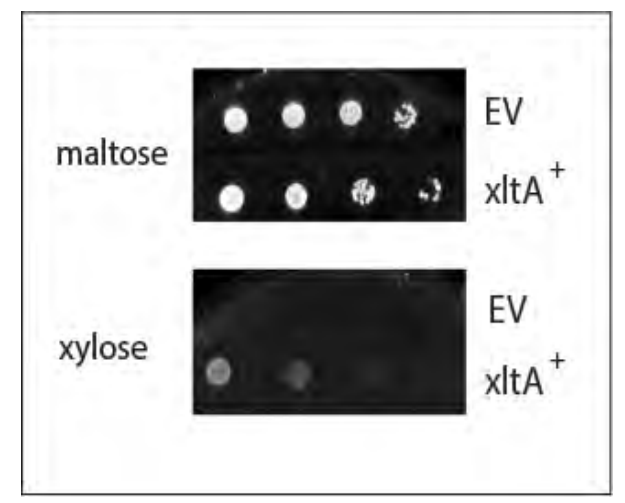

FIGURE 6.2. Functional validation of A. niger XltA as a xylose transporter, and of yeast strain EBY.XP as a xylose utilizing strain. Serial dilutions of the EBY.XP xltA ${ }^{+}$strain, expressing the $A$. niger sugar porter gene cloned in expression vector p426HXT7-6His, were grown in minimal medium agar plates containing maltose or xylose $1 \%(\mathrm{w} / \mathrm{v})$. Agar plates were incubated at $30^{\circ} \mathrm{C}$ for 5 days. EV: EBY.XP, carrying the empty expression vector p426HXT7-6His, was used as a negative control.

transformed with the p426HXT7-6His-xltA plasmid. Two Ag11C3 derived control strains were also constructed, one carrying the empty vector p426HXT7-6His, and one carrying the plasmid p426HXT7-6His-mstG, expressing a recently characterized glucose transporter from A. niger (Sloothaak et al., 2015). Four transformants per strain were isolated and, as can be observed in Figure 6.3, only transformants carrying the p426HXT7-6His-xltA plasmid were able to grow on xylose. This result confirmed that the laboratory-evolved strain Ag11C3 can be used as host for the identification of transporters able to transport xylose. It also showed that the recently identified $A$. niger $\mathrm{MstG}$ glucose transporter is unable to support growth on xylose in this strain.

\subsubsection{Functional validation of A. niger AnXItA-C and T. reesei TrStr1-3 in yeast}

Strain Ag11C3 was used to study the function of the selected A. niger and T. reesei sugar porters. Plasmids expressing $x l t B, x l t C$, str 1 , str2 or str3 were constructed using the p426HXT7-6His plasmid backbone and used to transform Ag11C3. Single colony transformants were isolated from minimal medium agar plates containing $2 \%$ maltose and the ability of XltA, XltB, XltC, Str1, Str2 and Str3 to support growth of the Ag11C3 transformant strains in the presence of xylose 


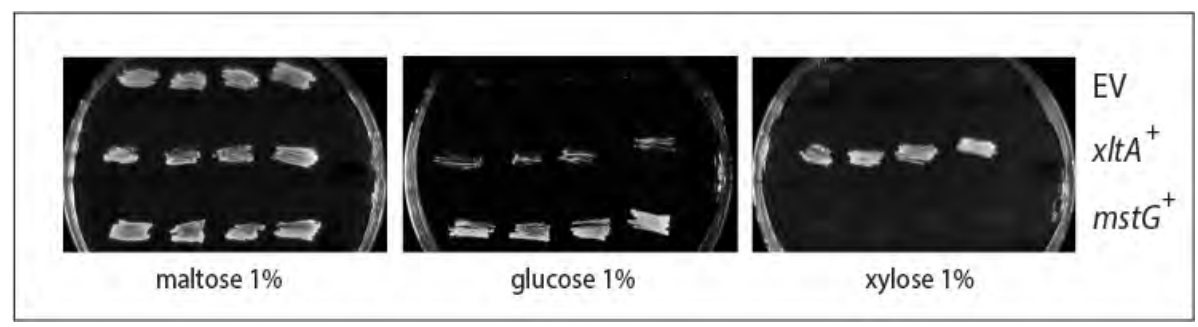

FIgURE 6.3. Functional validation of Ag11C3, a laboratoryevolved growth optimized derivative of the yeast strain EBY.XP. Growth of strain Ag11C3 expressing the A. niger xltA or $m s t G$ genes; or harboring the empty expression vector $\mathrm{p} 426 \mathrm{HXT7}$ $6 \mathrm{His}(\mathrm{EV})$, in minimal medium agar plates containing maltose, glucose, or xylose $1 \%(\mathrm{w} / \mathrm{v})$. Four transformants per genetic background were tested. Agar plates were incubated at $30{ }^{\circ} \mathrm{C}$ for 7 days.

and in the presence of a number of other monosaccharides was studied using a plate assay. Ten-fold serial dilutions of exponentially growing transformant cells were spotted on minimal medium plates supplemented with $1 \%(\mathrm{w} / \mathrm{v})$ or $0.1 \%$ (w/v) of one of the following carbon sources: xylose, glucose, fructose, galactose, mannose and maltose (Figure 6.4). All six previously selected transporters were functional as xylose transporters, as they all provided the Ag11C3 strain the ability to grow in xylose. Remarkable differences in the growth levels of the various transformants were observed, suggesting that the affinity of the transporters towards the pentose could be different. XltA, XltC and Str3 showed a good growth level in both high ( $1 \%$; w/v) and low $(0.1 \%$; w/v) concentrations of xylose after 7 days, XltB and Str2 transformants showed a lower growth level in both xylose concentrations after the same incubation period, while growth of the Str1 transformant could only be clearly observed after a prolonged incubation time of 12 days. In addition, most of the transporters showed the ability to transport other monosaccharides. XltC, Str2 and Str3 were able to support growth of the Ag11C3 strain in the presence of all sugars tested, showing them to be the transporters with the broadest substrate specificity. XltA and Str1 showed a poor performance in the use of fructose as substrate but restored growth of Ag11C3 in the presence of glucose, galactose and mannose. The most remarkable substrate utilization profile was that of the Ag11C3-XltB transformant, which was only able to grow in the presence of xylose.

For a better insight in individual xylose affinities, the growth rates of transformants expressing XltA, XltB, XltC, Str1, Str2 and Str3 were studied in liquid 


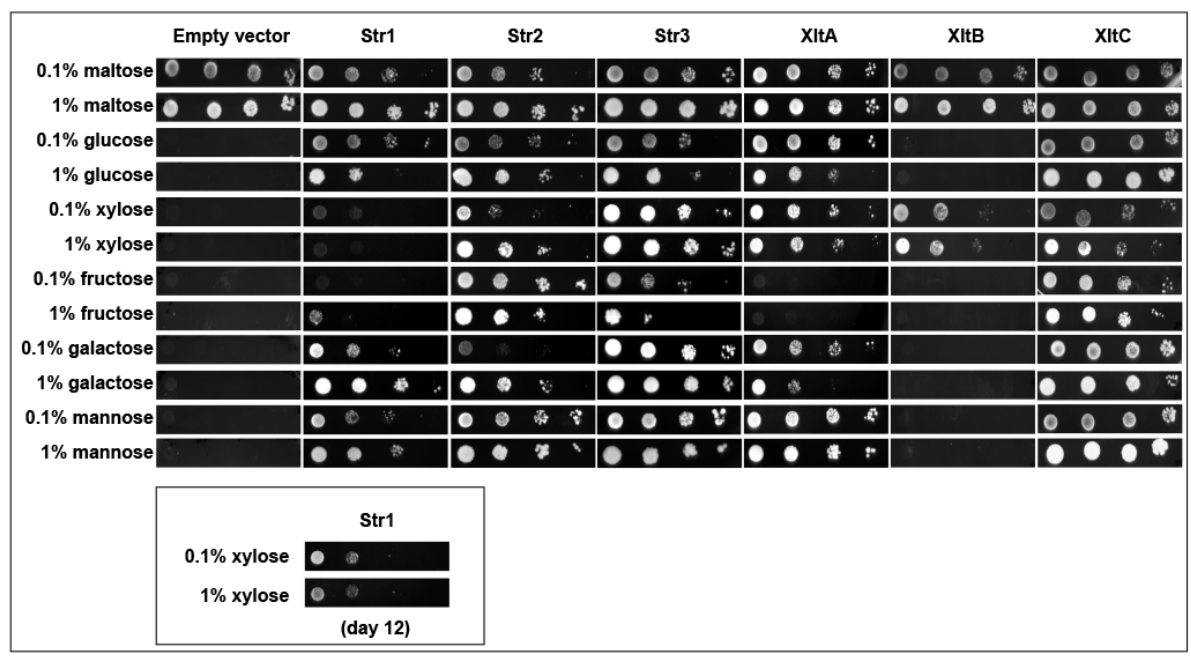

FIGURE 6.4. Heterologous expression and substrate utilization analysis of the $A$. niger sugar transporters XItA-C and T. reesei sugar transporters Str1-3. Ag11C3 transformants expressing str 1 , str2, str3, xltA, $x l t B$ or $x l t C$, were grown for 7 days at $30{ }^{\circ} \mathrm{C}$ in minimal medium agar plates containing a final concentration of $1 \%$ $(\mathrm{w} / \mathrm{v})$ or $0.1 \%(\mathrm{w} / \mathrm{v})$ of the following sugars: maltose, glucose, xylose, fructose, galactose and mannose. EV: Ag11C3, carrying the empty expression vector p426HXT7-6His. Insert: str 1 transformant grown for 12 days on xylose plates.

cultures in the presence of xylose $(0.5 \% ; \mathrm{w} / \mathrm{v})$ as sole carbon source, and in the presence of a mixture of xylose $(0.5 \% ; \mathrm{w} / \mathrm{v})$ and glucose $(0.5 \%$; w/v) (Table 6.1). Additional file 6 shows growth curves of the transformant strains grown in minimal medium with the described sugar compositions. As was already observed in the plate assays, all transformant strains were able to grow in the presence of xylose as sole carbon source. When comparing sugar uptake with growth curves, differences could be observed between the different transformants. With exception of the Str1 strain, in the presence of xylose as sole carbon source growth started immediately after inoculation. In concord to what has been observed in the plate assay, growth of Str1 transformant strain was observed only after ten days. Some xylose consumption was observed by HPLC analysis, but due to the long lag time and poor growth this could not be properly quantified. Together, these results suggest that the xylose transport capacity of Str1 transporter is very low. In contrast, the Str3 transformant showed the fastest growth rate in the presence of the pentose, followed by XltC, Str2, XltB and XltA. At the end 
of the culturing period Str3 had consumed all xylose present in the media. Str2, XltC and XltB had used $50 \%$ to $57 \%$ of the available xylose, while XltA used the $34 \%$ (Table 6.1).

Table 6.1: Specific growth rate and sugar consumption by Ag11C3 transformants expressing XltA, XltB, XltC, Str1, Str2 and Str3. $\mu=$ specific growth rate, NQ= not quantifiable, ND = not detectable, ${ }^{*}$ Long lag phase on xylose

\begin{tabular}{lccccc}
\hline $\begin{array}{l}\text { Ag11C3 } \\
\text { strain }\end{array}$ & \multicolumn{2}{c}{ Xylose cultures } \\
& $\mu\left(\mathrm{h}^{-1}\right)$ & $\begin{array}{c}\text { Total xylose } \\
\text { consumed }(\%)\end{array}$ & $\mu\left(\mathrm{h}^{-1}\right)$ & $\begin{array}{c}\text { Xylose + Glucose cultures } \\
\text { Total xylose } \\
\text { Total glucose } \\
\text { consumed }(\%)\end{array}$ \\
consumed $(\%)$
\end{tabular}

In the presence of the xylose and glucose mixture $(0.5 \%+0.5 \%$; w/v), each of the transformant strains behaved differently. In comparison to xylose cultures, growth of the Str1, Str2, Str3 and XltC transformant strains was enhanced by the addition of glucose, whereas growth of the XltB transformant was similar, and the XltA transformant grew less. This can be explained by different affinities of the respective sugar porters towards glucose and xylose. Str2 and Str3 transformant strains were able to use glucose efficiently (Table 6.1). In the presence of glucose, no xylose uptake could be determined with the Str2 transformant, whereas the Str3 transformant was able to use both carbon sources simultaneously. Xylose uptake by the XltC transformant was inhibited by glucose as well, but utilization of the hexose was not as efficient as observed for Str2 and Str3 (Table 6.1). In the presence of glucose Str1 initially grew faster, suggesting that Str1 could also transport glucose in the presence of the pentose, but growth levels when reaching the stationary phase were lower than observed in the cultures with xylose. Again sugar utilization could not be properly monitored by HPLC analysis suggesting a very low rate of glucose uptake as well.

When compared to the xylose medium, the growth rate of the Ag11C3 transformants expressing $A$. niger XltA and XltB was not higher in the mixed xyloseglucose medium and, in contrast to the T. reesei Str2 and Str3 transporters, glucose transport by $A$. niger XltA and XltB was not efficient. Moreover, the overall carbon uptake (glucose + xylose) in the sugar mix cultures was lower than that one observed for the xylose cultures (Table 6.1). This was also the case for the Ag11C3-XltC transformant. In the presence of glucose XltA was unable to transport xylose, while glucose was transported at a low rate (even lower than the xylose transport levels when the pentose was used as the sole carbon 
source). Inhibition of a xylose specific transporter by glucose has been previously reported for the well-characterized XylE, from E. coli (Sun et al., 2012). Even more remarkable was the performance of the XltB transformant in the mixed xylose-glucose medium: in the presence of glucose, xylose uptake was reduced but still two times higher than the uptake of glucose. Together with the plate assay study (Figure 6.4), these results strongly suggest that XltB is a transporter specific for xylose.

In order to get more insights about both the biochemical properties and the biological role of these transporters, radiolabelled sugar uptake studies and transcriptional analysis of the respective coding genes was performed.

\subsubsection{Analysis of uptake kinetics by ${ }^{14} \mathrm{C}$-labelled sugar uptake studies}

The growth data presented above effectively demonstrated that all the candidate transporters are able to transport xylose. Differences observed in growth rate and sugar uptake of yeast transformants expressing individual transporters, suggested important differences in substrate specificity and uptake rate. We therefore measured the kinetics of xylose and glucose uptake of the six transporters. Transport assays were performed using a range of $\mathrm{D}-\left[1{ }^{14} \mathrm{C}\right]$-xylose and $\mathrm{D}-\left[\mathrm{U}-{ }^{14} \mathrm{C}\right]$-glucose concentrations. Initial uptake rates were calculated, fitted to the Michaelis-Menten model and used to estimate the appropriate kinetic parameters $\left(\mathrm{K}_{\mathrm{m}}\right.$ and $\left.\mathrm{V}_{\max }\right)$ as previously described (Derntl et al., 2013) (Table 6.2) (Additional file 7).

The affinity for xylose of the six transporters ranged from $90 \mu \mathrm{M}$ (XltA) to 15 $\mathrm{mM}$ (XltB), being, in most cases, higher than those reported for most of the fungal xylose transporters characterized to date, with values between $80 \mu \mathrm{M}$ and 150 mM (Du et al., 2010; Leandro et al., 2006; Vankuyk PA et al., 2004; Weierstall et al., 1999). A. niger XltA showed to have a very high affinity for xylose $(0.09 \pm$ $0.03 \mathrm{mM}$ ), which is even higher than reported for the $E$. coli xylose transporter XylE (0.47 mM) (Davis and Henderson, 1987; Farwick et al., 2014), and the $A$. niger high affinity sugar transporter MstA $(0.3 \pm 0.1 \mathrm{mM})$ (Vankuyk PA et al., 2004). The transporters XltC (4.71 $\pm 1.04 \mathrm{mM})$, Str1 (5.70 $\pm 0.19 \mathrm{mM})$, Str2 $(6.18 \pm 0.81 \mathrm{mM})$ and Str3 $(2.19 \pm 0.29 \mathrm{mM})$ showed a high affinity towards xylose within the same order of magnitude, whereas XltB $(15.00 \pm 4.50 \mathrm{mM})$ had a slightly lower affinity. Regarding glucose transport characteristics, five out of six transporters showed a very high affinity for the hexose, ranging from $13 \mu \mathrm{M}$ (Str1) to $108 \mu \mathrm{M}$ (XltC). These are, in all cases, in the same range as 
Table 6.2: Xylose and glucose initial uptake kinetics of fungal MFS transporters. + = Two glucose transport components were reported, ND = not detectable, $-=$ not determined, $\mathrm{NC}=$ not comparable $(* *$ values were reported in an incompatible unit (nmol $\mathrm{min}^{-1} \mathrm{mg}$ protein ${ }^{-1}$ ), Ag11C3 = laboratory evolved xylose utilizing strain transformed with the empty vector p426HXT7-6His, transporters in bold originate from filamentous fungi.

\begin{tabular}{|c|c|c|c|c|c|}
\hline \multirow[t]{2}{*}{ Transporter } & \multicolumn{2}{|c|}{ Xylose initial uptake kinetics } & \multicolumn{2}{|c|}{ Glucose Initial uptake kinetics } & \multirow[t]{2}{*}{ Reference } \\
\hline & $\begin{array}{c}\mathrm{V}_{\max } \\
\left(\mathrm{nmol} \mathrm{min}^{-1} \mathrm{mg} \mathrm{DW}^{-1}\right)\end{array}$ & $\mathrm{K}_{\mathrm{m}}(\mathrm{mM})$ & $\begin{array}{c}\mathrm{V}_{\max } \\
\left(\mathrm{nmol} \mathrm{min}^{-1} \mathrm{mg} \mathrm{DW}^{-1}\right)\end{array}$ & $\mathrm{K}_{\mathrm{m}}(\mathrm{mM})$ & \\
\hline AnXItA & $1.08 \pm 0.05$ & $0.09 \pm 0.03$ & $1.11 \pm 0.15$ & $0.07 \pm 0.01$ & This study \\
\hline AnXItB & $0.10 \pm 0.00$ & $15.0 \pm 4.50$ & ND & ND & This study \\
\hline AnXltC & $0.14 \pm 0.01$ & $4.71 \pm 1.04$ & $1.18 \pm 0.15$ & $0.11 \pm 0.02$ & This study \\
\hline TrStr1 & $0.04 \pm 0.01$ & $5.70 \pm 0.19$ & $0.14 \pm 0.04$ & $0.01 \pm 0.00$ & This study \\
\hline TrStr2 & $0.12 \pm 0.02$ & $6.18 \pm 0.81$ & $0.69 \pm 0.19$ & $0.05 \pm 0.01$ & This study \\
\hline TrStr3 & $0.46 \pm 0.04$ & $2.19 \pm 0.29$ & $1.31 \pm 0.33$ & $0.06 \pm 0.01$ & This study \\
\hline AnMstA & $\mathrm{NC}^{* *}$ & $0.3 \pm 0.1$ & $\mathrm{NC}$ & $0.03 \pm 0.01$ & (Vankuyk PA et al., 2004) \\
\hline GXS1 & $0.01 \pm 0.00$ & $0.08 \pm 0.02$ & - & - & (Young et al., 2012) \\
\hline XUT3 & $0.01 \pm 0.00$ & $4.09 \pm 1.08$ & - & - & (Young et al., 2012) \\
\hline Хур29 & $0.61 \pm 0.05$ & $175.74 \pm 21.36$ & ND & ND & (Du et al., 2010) \\
\hline An25 & $0.69 \pm 0.04$ & $55.96 \pm 9.37$ & ND & ND & (Du et al., 2010) \\
\hline SUT1 & $132.0 \pm 1.0$ & $145.0 \pm 1.0$ & $45.0 \pm 1.0$ & $1.5 \pm 0.1$ & (Weierstall et al., 1999) \\
\hline SUT2 $^{+}$ & $41.0 \pm 1.0$ & $49.0 \pm 1.0$ & $\begin{array}{r}3.3 \pm 0.1 \\
28.0 \pm 4.0\end{array}$ & $\begin{aligned} 1.1 & \pm 0.1 \\
55 & \pm 11.0\end{aligned}$ & $\begin{array}{l}\text { (Weierstall et al., 1999) } \\
\text { (Weierstall et al., 1999) }\end{array}$ \\
\hline SUT3 $^{+}$ & $87.0 \pm 2.0$ & $103.0 \pm 3.0$ & $\begin{array}{r}3.7 \pm 0.1 \\
22.0 \pm 0.1\end{array}$ & $\begin{array}{r}0.8 \pm 0.1 \\
31.0 \pm 0.1\end{array}$ & $\begin{array}{l}\text { (Weierstall et al., 1999) } \\
\text { (Weierstall et al., 1999) }\end{array}$ \\
\hline Ag11C3 & ND & ND & ND & ND & This study \\
\hline
\end{tabular}

reported for other A. niger high-affinity glucose transporters (Sloothaak et al., 2015; Vankuyk PA et al., 2004). It is a common feature of reported glucose/xylose transporters to show higher affinity for the hexose than for the pentose (Du et al., 2010), with differences of around two orders of magnitude (Farwick et al., 2014). This was observed for the three T. reesei transporters characterized in this study, while the $A$. niger XltC affinity towards glucose was around 50 times higher than towards xylose (Table 6.2). However, A. niger XltA showed approximately the same high affinity for xylose $(0.09 \pm 0.03 \mathrm{mM})$ as for glucose $(0.07 \pm 0.01 \mathrm{mM})$. Also, XltA was able to transport both sugars at the same rate. In contrast, uptake of radiolabelled glucose by XltB was not detected, as was previously described for the $P$. stipitis Xyp29 and the $N$. crassa An25 xylose transporters (Du et al., 2010). This result was in concordance with the inability of XltB to support the growth of the Ag11C3 strain in the presence of glucose as a sole carbon source. The biochemical characteristics of the six studied transporters, plus those reported previously (Sloothaak et al., 2015; Vankuyk PA et al., 2004; Zhang et al., 2013), revealed the wide range of glucose and xylose uptake systems featured by these fungi. The initial uptake kinetics of both sugars displayed by XltA and XltB suggested a possible biological role for these transporters in xylose utilization. 


\subsubsection{Transcriptional analysis of T. reesei str1-3 and A. niger xltA-C}

In order to shed more light on their possible biological role in xylose uptake, a transcriptional analysis, performed via RT-qPCR of the respective transporter coding genes was done. For this, the expression of $A$. niger $x l t A, x l t B$ and $x l t C$; and $T$. reesei str1, str2 and str3 was studied, in different culture conditions, in both wild type and $x \ln R / x y r 1$ mutant strains which harbor an inactivated transcriptional activator of the xylanolytic system (Figure 6.5). The samples for expression analysis of the $A$. niger genes were obtained from mycelium grown in cultures containing minimal medium plus $55 \mathrm{mM}$ glucose, $66 \mathrm{mM}$ xylose, $0.5 \mathrm{mM}$ xylose, or no carbon source (NCS), whereas T. reesei samples were isolated from cultures on minimal medium containing one of the following carbon sources: 55 $\mathrm{mM}$ glucose, $66 \mathrm{mM}$ xylose, $1.5 \mathrm{mM}$ sophorose, or NCS.

In case of the $A$. niger transporter genes $x l t A$ was strongly induced by high xylose concentrations, and its expression levels were also significantly higher in lower xylose concentrations when compared to the rest of the conditions studied (Figure 6.5). XlnR is a transcriptional activator of the xylanolytic system in $A$. niger (van Peij et al., 1998). In the $x \ln R$ mutant strain, $x l t A$ transcript levels dropped dramatically in the presence of low and high xylose concentrations, while they were kept at the same level in the NCS and glucose conditions. This indicates a clear role of XlnR in the transcriptional regulation of $x l t A$, and thus, a role of XltA in xylose uptake by $A$. niger. The $x l t B$ gene expression was apparently not xylose dependent as it was repressed in the presence of high concentrations of both glucose and xylose, and its expression levels in the presence of low xylose concentrations were slightly lower than in the NCS condition. Also, the expression profile of $x l t B$ was similar in the wild type and the $\Delta x \ln R$ strain. Therefore a role of $\mathrm{X} \ln R$ in the regulation of this gene in the studied conditions could be discarded. According to $x l t B$ expression profile, XtlB seems to be relevant in A. niger when there is a low availability of carbon sources in the environment. In the uptake kinetics studies, the transport of low xylose concentrations by XltB could be determined, but it was not possible for low glucose concentrations. Thus, the substrate specificity, and the higher affinity for xylose than for glucose shown by XltB, together with its preferential expression at low carbon source concentrations, indicate that this transporter could have a role for xylose transport in the fungus independent of XlnR activation. The expression levels of $x l t C$ were, in contrast, higher when high sugar concentrations were present in the medium. They were particularly high in the xylose $66 \mathrm{mM}$ 

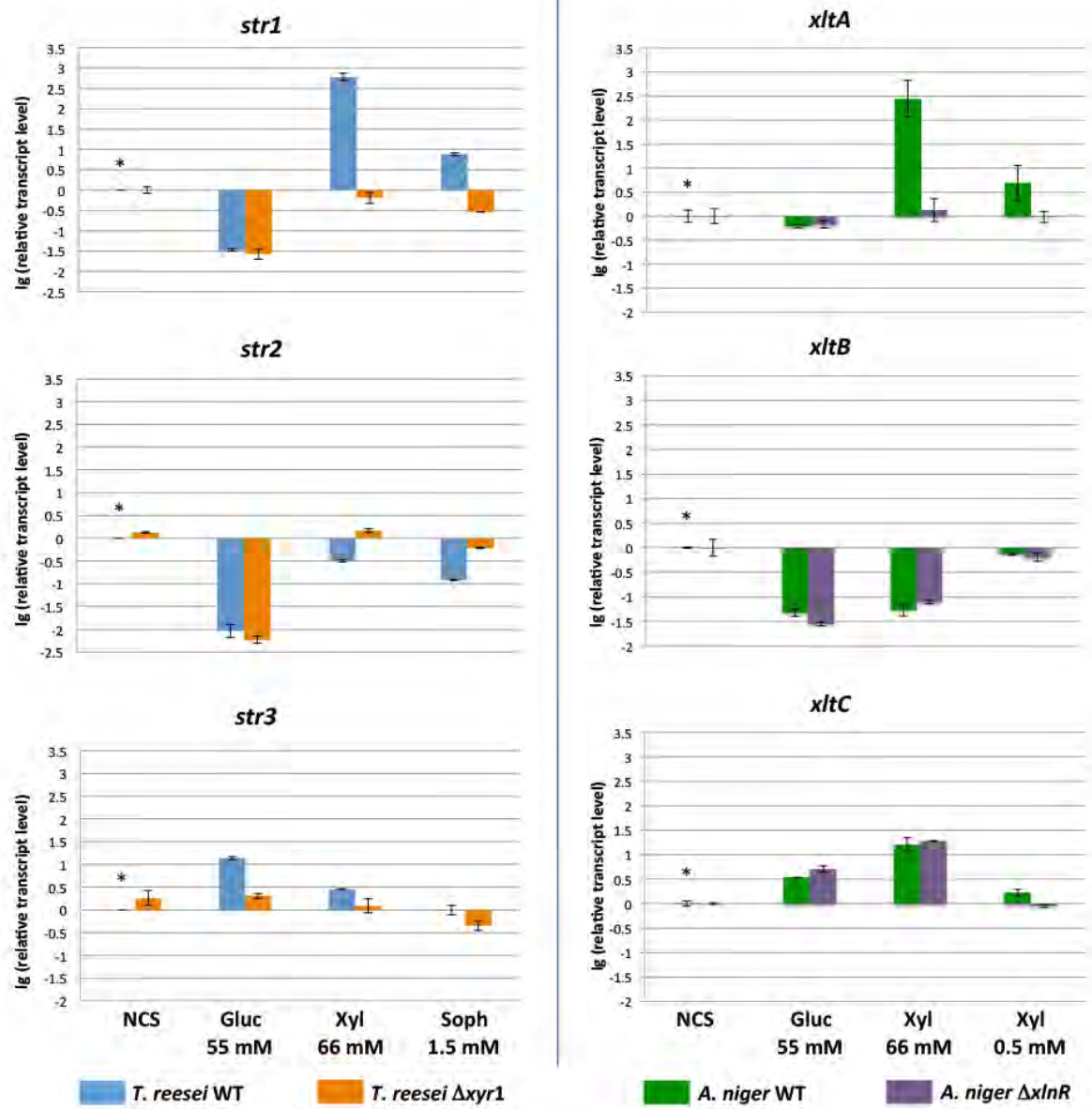

FiguRE 6.5. Transcriptional analysis of $T$. reesei str1-3 and $A$. niger $\boldsymbol{x l t A}$ - $\boldsymbol{C}$. Samples were taken $3 \mathrm{~h}$ after mycelium transfer to the different culture conditions, and expression analyses were performed by RT-qPCR. Normalization of the expression data was done using the histone-like gene "hist" transcript (gene ID 207921) for A. niger, and genes sar1 and act1 for T. reesei. Results are given as relative transcript ratios in logarithmic scale (lg). The values provided in the figures are means of two biological replicates. Transcript levels always refer to the reference sample, indicated with an asterisk.

condition. The role of $\mathrm{XlnR}$ on the transcriptional regulation of this gene was not clear, as its expression levels in the mutant strain were only slightly reduced in the xylose $0.5 \mathrm{mM}$ condition, but not reduced at all in the xylose $66 \mathrm{mM}$ condition. 
Regarding the $T$. reesei genes, str 1 expression was strongly induced by xylose, in a similar way to $A$. niger $x l t A$, and also by sophorose, which is also an inducer of the T. reesei xylanolytic system (Derntl et al., 2013). In addition, it was clearly transcriptionally regulated by $\mathrm{Xyr} 1$, encoding the main regulator of the xylose metabolism in T. reesei (Stricker et al., 2006). Despite Str1 low efficiency in xylose transport, the transcriptional behavior of its coding gene, and the recent findings reported by Huang et al. (2015), suggest an important role for the transporter in xylose utilization (Huang et al., 2015). In the wild type and $\Delta x y r 1$ strains, str2 was preferentially expressed in resting cell conditions and strongly down-regulated in the presence of $55 \mathrm{mM}$ glucose. In case of the xylose and sophorose conditions, str2 expression levels were slightly higher in the xyr 1 deletion strain. The str3 gene did not seem to be specifically induced by xylose, being its expression levels higher in the presence of glucose (Figure 6.5 ). In the absence of Xyr1 ( $\Delta x y r 1$ strain), str3 expression levels dropped in the presence of xylose and sophorose, but the same was observed in the glucose condition, while its expression was slightly increased when no carbon source was present. Although a role of Xyr1 in str3 regulation cannot be discarded, the obtained results are not conclusive enough. The higher str3 expression levels observed in the presence of $66 \mathrm{mM}$ xylose and especially in $55 \mathrm{mM}$ glucose when compared to low concentrations of sophorose and the NCS condition (wild type strain), do suggest a role for Str3 in the uptake of high sugar concentrations. This hypothesis is also in agreement with the glucose and xylose transporting behavior shown by the Str3 expressing yeast strain constructed in this study.

\subsubsection{Characteristics defining AnXItA-C and TrStr1-3 xylose transporters}

Xylose was a substrate for all 6 different transporters, suggesting that the $\mathrm{HMM}_{\mathrm{xylT}}$ may have captured residues discriminating for glucose-xylose porters. With the aim of pinpointing these discriminating residues, that should be conserved in the novel xylose transporter proteins, we used a MSA of all transporters used for $\mathrm{HMM}_{\text {gluT }}$ (constructed in a previous study, (Sloothaak et al., 2015)) and $\mathrm{HMM}_{\text {xylT }}$ (this study), plus the newly identified A. niger and T. reesei transporters (Additional file 8). A number of motifs and residues from fungal sugar porters have been recently reported to be relevant for xylose transport by different studies, and the amino acid sequences of AnXltA-C and TrStr1-3 were analyzed for the presence of these motifs (Figure 6.6). Wang and collaborators suggested the relevance of the aromatic residue enriched motif YFFYY (332-336), present in 
the transmembrane section 7, for the xylose transport capacity of Mgt05196p from Meyerozyma guilliermondii (Wang et al., 2015). TrStr3, which showed the highest xylose transport capacity in this study, also contains the YFFYY (320$324)$ peptide. The same motif is present as well in a number of sugar porters (MSA), but it is not completely conserved in transporters that are exclusive for xylose as XylE from E. coli, An25 from N. crassa or Xyp29 (also called Xut6 and Stl12) from $P$. stipitis: the first three aromatic residues from the YFFYY motif are substituted by aliphatic ones in XylE and An25. This was also found to be the case in AnXtlB (ALIYY); AnXltC (VMMYY) (although not strictly an aliphatic residue, methionine is usually considered as such, since its sulfur group is not reactive); TrStr1 (AVLYY); and TrStr2 (ALIYY). In addition, the same motif in AnXltA (AINYY) had a polar residue (N) at the third position. Although most of the functionally validated sugar transporters contain a nonpolar (aromatic or aliphatic) amino acid at that position, several sugar porters, including the xylose transporters Hxt2.6 (LVSYY) and Xyp 29 (IITYY) from P. stipitis, contain a polar residue as well. The Mgt05196p residues aspartate D72 and arginine R164, also suggested to be crucial for xylose transport (Wang et al., 2015), were found to be conserved in AnXltB, AnXltC, TrStr2 and TrStr3. AnXltA and TrStr1 had the arginine conserved, but both contained an asparagine residue at the aspartate 72 of Mgt05196p. Since asparagine and aspartate are similar amino acids, both might have the same function at that particular position.

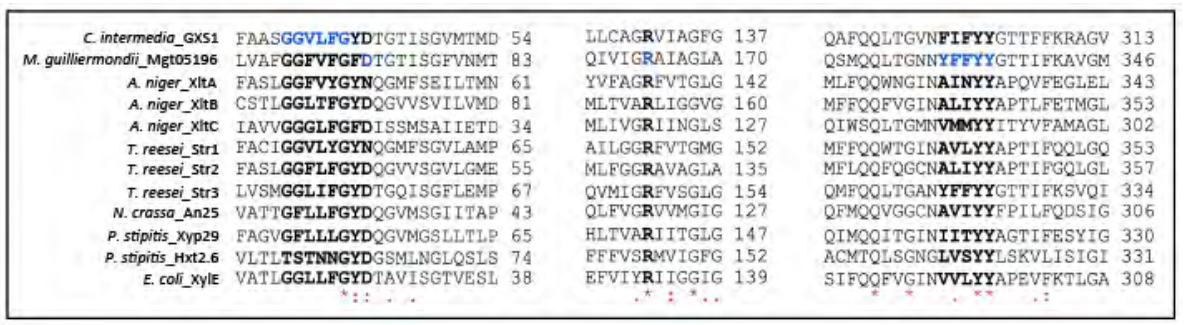

FIGURE 6.6. Conservation of motifs and residues reported to be relevant for xylose transport. A multiple sequence alignment of known xylose transporters, including $A$. niger XltA-C and T. reesei Str1-3 was constructed using the EMBL-EBI Clustal Omega tool (Sievers et al., 2011). Reported relevant motifs and residues are in bold, amino acids highlighted in blue indicate the transporter where motifs/residues were described to be relevant for xylose transport.

The motif GG/FXXXG, present at the first transmembrane span of sugar porters and highly enriched in those that confer growth on xylose (Young et al., 
2014), was also found to be present in AnXltA-C and TrStr1-3 (Figure 6.6). Knoshaugh and collaborators (2015) recently highlighted the variability of this motif, that allow to distinguish between xylose transporters (GGLXXGYD/N), arabinose transporters (XGXXFGFD) and glucose transporters (GGFXFGWG) (Knoshaug et al., 2015). This motif was subjected to protein engineering through saturation mutagenesis in the $C$. intermedia glucose-xylose symporter 1 (GXS1) (Young et al., 2014). In GXS1, the wild-type motif is GGVLFG (36-41). Saturation mutagenesis for each of the three variable residues (V38, L39, F40) produced changes in selectivity and efficiency of monosaccharide transport by GXS1. Residues V38 and F40 were found to be involved in carbon source selectivity, and L39 in controlling substrate transport efficiency. Interestingly, some of the most significant V38 substitutions were also found in the here studied transporters. $\mathrm{V} 38 \mathrm{~F}$, that almost completely attenuated glucose exponential growth rate while amplifying exponential xylose growth rate by $50 \%$, was found in XltA and Str2. V38L, that increased the exponential xylose growth rate by $73 \%$ without altering glucose exponential growth rate significantly, was found in XltB and Str3. V38G, which also produced a positive effect on xylose transport, was found in XltC. The L39 substitutions resulted in a general or differential attenuation of GXS1 transport function. L39I, found to be responsible for a specific attenuation of glucose transport in favor of xylose transport, was found in Str3. The L39V substitution, present in all three $P$. stipitis high-velocity xylose transporters SUT1-3 (Weierstall et al., 1999), was also found in XltA. Regarding the GXS1 F40 residue, XltB, XltC, Str2 and Str3 have the same amino acid at the same position, whereas XltA and Str1 contain a Y. The F40Y substitution in GXS1 produced an attenuating effect on the transport of xylose and other monosaccharides, indicating that the particular tyrosine residue could have the same role in XltA and Str1. This could explain, in part, the low efficiency displayed by Str1 on xylose transport, and provides a hint on how the transport capacity of both transporters could be improved.

Regarding the presence of amino acids that have been shown to be key at certain positions for glucose affinity in yeast sugar porters, like the threonine 219/213 and the asparagine 376/370 of $S$. cerevisiae Gal2/Hxt7 (Farwick et al., 2014; Kasahara et al., 2011), interesting features were also found. Only XltC, Str2 and Str3, that were the most efficient glucose transporters in the presence of xylose (Table 6.1), keep both residues conserved; while XltB and Str1, that showed the lowest glucose transport capacity, have different residues in both positions.

In summary, the amino acid sequence analysis of the six transporters showed 
that all of them harbor motifs and residues previously associated to fungal xylose transporters. This fact, in combination with the experimental evidences provided, indicates a role of these transporters in xylose uptake.

\subsection{Conclusions}

In this study, computational and experimental approaches were successfully combined for the identification and characterization of xylose transporting proteins from the industrial cell factories $A$. niger and T. reesei. Comparing the $\mathrm{HMM}_{\mathrm{xylT}}$ output with recently published transcriptome studies, also taking into account phylogenetic distance relationships, was a good strategy to link a specific group of MFS porters with the utilization of lignocellulosic feedstocks. By using the mentioned methodology, five putative xylose transporters (XltA, XltB, XltC, Str2, Str3), and the recently identified xylose transporter Str1, were selected and successfully validated as xylose transporters. All of them displayed significant differences in their substrate specificity and biochemical properties, being XltA and XltB of particular interest, due to the high affinity for xylose of the former, and the narrow substrate specificity of the latter. Also, new insights about the regulation, at transcriptional level, of xylose utilization by A. niger and T. reesei were found, the most remarkable being that xylose uptake is not completely controlled by the XlnR/Xyr1 regulon.

To the best of our knowledge, this is the first study on the functional validation and characterization of sugar porters with their biological role specifically associated to xylose transport in $A$. niger. Also, the biochemical characterization of xylose transporters in T. reesei is reported for the first time. In summary, this study contributes to a better understanding of xylose utilization by two relevant industrial filamentous fungi, and provides new tools for strain engineering in fungi.

\subsection{Methods}

\subsubsection{Construction of a xylose hidden Markov model}

The protein sequences used to build $\mathrm{HMM}_{\mathrm{xylT}}$ were obtained from the UniProt database (Consortium et al., 2014), and aligned using the PRALINE structural alignment tool (Simossis and Heringa, 2005) with the same parameters as described for the hidden Markov model constructed in our previous work (Sloothaak et al., 2015). HMM ${ }_{\text {xylT }}$ was built using the HMMER v3.0 tool (Finn et al., 2011). The A. niger ATCC1015 (Andersen et al., 2011) and the T. reesei Rut-C30 (Koike 
et al., 2013) proteomes, which were used for the in silico analysis, were downloaded from the JGI database (Nordberg et al., 2013).

\subsubsection{Strains and growth conditions}

Escherichia coli DH5 $\alpha$ (endA1, hsdR17, gyrA96, thi-1, relA1, supE44, recA1, $\triangle l a c U 169$ ( $(80$ lacZ $\Delta \mathrm{M} 15)$ ) was used for cloning experiments and plasmid propagation. It was grown at $37^{\circ} \mathrm{C}$ on $\mathrm{LB}$ medium ( $1 \%$ tryptone, $0.5 \%$ yeast extract, $1 \% \mathrm{NaCl} ; \mathrm{w} / \mathrm{v})$, with $100 \mu \mathrm{g} \mathrm{ml}^{-1}$ ampicillin when required for transformants selection.

A. niger N400 (CBS 120.49), NW199 (fwnA6, leuA5, goxC17, pyrA6; $\triangle x \operatorname{lnR}$ : :pIM240) (Hasper et al., 2004) and T. reesei QM6a $\Delta$ tmus53 (ATCC 13631) (Steiger et al., 2011) and QM6a $\Delta t m u s 53 \Delta x y r 1$ (ATCC 13631) (Mello-de Sousa et al., 2015) strains were used in mycelium transfer experiments for the MFS genes transcriptional analysis. The $A$. niger and $T$. reesei strains were maintained on complete medium agar (Pontecorvo et al., 1953) and malt extract agar (MEX) respectively, at $30^{\circ} \mathrm{C}$.

Mycelium transfer experiments of both fungal species were performed in a similar way, using liquid cultures in Erlenmeyer flasks on a rotary shaker. The A. niger strains were pre-cultured at $30^{\circ} \mathrm{C}$ and $200 \mathrm{rpm}$, during 18 hours, in minimal medium containing $4.50 \mathrm{~g} \mathrm{~L}^{-1} \mathrm{NaNO}_{3}, 1.13 \mathrm{~g} \mathrm{~L}^{-1} \mathrm{KH}_{2} \mathrm{PO}_{4}, 0.38 \mathrm{~g} \mathrm{~L}^{-1}$ $\mathrm{KCl}, 0.38 \mathrm{~g} \mathrm{~L}^{-1} \mathrm{MgSO}_{4} \cdot 7 \mathrm{H} 2 \mathrm{O}, 750 \mu \mathrm{L} \mathrm{L}^{-1}$ of Vishniac solution, and $100 \mathrm{mM}$ sorbitol (Pontecorvo et al., 1953; Vishniac and Santer, 1957). Equal amounts of water-rinsed mycelium were transferred to minimal medium with the following carbon source compositions: $55 \mathrm{mM}$ D-glucose, $66 \mathrm{mM}$ D-xylose, $0.5 \mathrm{mM}$ D-xylose or no carbon source (NCS). The initial $\mathrm{pH}$ of the medium in all conditions was set at 6.0. The T. reesei strains were pre-cultured in Mandels-Andreotti (MA) medium (Mandels, 1985), containing $1 \%(\mathrm{w} / \mathrm{v})$ glycerol as the sole carbon source, at $30{ }^{\circ} \mathrm{C}$ and $180 \mathrm{rpm}$ for $22 \mathrm{~h}$. Pre-grown mycelia were washed, then equal amounts were resuspended in MA media containing $55 \mathrm{mM}$ D-glucose, $66 \mathrm{mM}$ D-xylose, $1.5 \mathrm{mM}$ sophorose or in medium without carbon source (NCS). In both experiments, three hours after mycelium transfer samples were taken and quickly washed, dried with a single-use towel, snap-frozen with liquid nitrogen and stored at $-80{ }^{\circ} \mathrm{C}$ until further processing. Two biological replicates per condition were studied in all cases.

The $S$. cerevisiae strain EBY.VW4000 (CEN.PK2-1C hxt13 $\triangle:: l o x P ;$ hxt15:: $\triangle$ loxP;

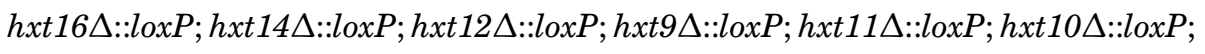

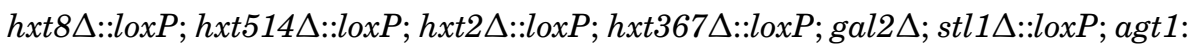


:loxP; ydl247w $\Delta:: l o x P ; y j r 160 c \Delta:: \operatorname{loxP}$ ) (Wieczorke et al., 1999), previously transformed with the plasmid pRH315 (Hector et al., 2011), expressing the D-xylose reductase and xylitol dehydrogenase from $P$. stipitis, and the $S$. cerevisiae xylulokinase, was used as a xylose utilising strain for the characterization of sugar transporters. It was grown at $30^{\circ} \mathrm{C}$ and maintained in solid complete medium containing $10 \mathrm{~g} \mathrm{~L}^{-1}$ of yeast extract, $20 \mathrm{~g} \mathrm{~L}^{-1}$ of peptone and $20 \mathrm{~g} \mathrm{~L}^{-1}$ of maltose. The EBY.VW4000 derived strains obtained in the present study were grown in liquid minimal medium containing $6.7 \mathrm{~g} \mathrm{~L}^{-1}$ of yeast nitrogen base with ammonium sulphate (Difco), $20 \mathrm{~g} \mathrm{~L}^{-1}$ of maltose, supplemented with leucine ( $30 \mathrm{mg} \mathrm{L}^{-1}$ ) and histidine $\left(20 \mathrm{mg} \mathrm{L}^{-1}\right)$. Growth rates $(\mu)$ of the Ag11C3 transformants, during time course cultivations, were calculated using the O.D. values obtained from $\mathrm{T}=$ 0 until the cultures reached stationary phase.

\subsubsection{Construction of S. cerevisiae Ag11C3 transformants expressing $A$. niger and $T$. reesei genes}

The coding sequence of the genes $x l t A, x l t B, x l t C$, str1, str2 and str3, was obtained through PCR amplification of $A$. niger and $T$. reesei cDNA samples respectively. The coding sequence of the gene $x l t A$, digested with SpeI and XhoI was cloned on the S. cerevisiae expression vector p426HXT7-6His (Hamacher et al., 2002), previously linearized with SpeI and XhoI, under the control of the constitutive promoter HXT7p and the terminator CYC1t. The $x l t B, x l t C$, str1, str2 and str3 cDNA sequences were amplified with oligonucleotides containing 40 additional base pairs, corresponding to the $\mathrm{p} 426 \mathrm{HXT} 7-6 \mathrm{His}$ cloning site, and were cloned to the vector through yeast mediated recombination (Schuster et al., 2012). Primer sequences and plasmids used in this study are provided in Additional file 9. The Ag11C3 yeast strain transformation was performed as previously described (Gietz and Woods, 2002).

The curing of the p426HXT7-6His-xltA plasmid, containing the URA3 selection marker, from the Ag11 strain was done by growing the transformant in minimal medium plates containing maltose and uracil, thereby relieving the plasmid selective pressure. After three re-plating rounds in maltose-uracil plates, p426HXT7-6His-xltA cured strains were isolated through their growth on minimal medium plates containing maltose, uracil and 5-fluoroorotic acid (FOA). FOA is a commonly used agent to select for the absence of the URA3 gene in yeast strains (Boeke et al., 1987). A single colony growing on the FOA plate, named $\mathrm{Ag} 11 \mathrm{C} 3$, was selected for its use as host for the expression of new xylose transporter candidates. 


\subsubsection{Sugar analyses}

Xylose and glucose present in the $S$. cerevisiae culture supernatants were quantified by high pressure liquid chromatography (HPLC) analysis. The samples were centrifuged at maximum speed in a benchtop centrifuge for 10 minutes and analysed on a Dionex ICS-5000+ instrument (Thermo Scientific), equipped with a CarboPac MA1 column. Separation was performed by isocratic elution with 480 $\mathrm{mM} \mathrm{NaOH}$, at a flow rate of $0.4 \mathrm{~mL} \mathrm{~min}^{-1}$ for 35 minutes.

\subsubsection{Analysis of uptake kinetics by ${ }^{14} \mathrm{C}$-labelled sugars uptake studies}

Sugars uptake assays were performed as described (Walsh et al., 1994), with minor adjustments. A pre-inoculum $(50 \mathrm{~mL})$ of Synthetic Enhanced medium (SE; $6.7 \%(\mathrm{w} / \mathrm{v})$ YNB w/o amino acids (Difco) $+20 \mathrm{mg} \mathrm{L}^{-1} \mathrm{~L}$-arginine and Lmethionine), containing appropriate amino acids, and $2 \%(\mathrm{w} / \mathrm{v})$ maltose as a carbon source, was inoculated and incubated for 48 hours $\left(30{ }^{\circ} \mathrm{C}, 225 \mathrm{rpm}\right)$. The pre-inoculum was then transferred to $200 \mathrm{~mL}$ of fresh SE-medium, and after 48 hours incubation, transferred to $500 \mathrm{~mL}$ fresh SE-medium. After 24 hours incubation, cells were harvested by centrifugation (4'000 g; $10 \mathrm{~min}$ ) and washed with $50 \mathrm{~mL}$ ice-cold ultrapure water. Cells were then washed and resuspended in ice-cold $100 \mathrm{mM}$ phosphate-buffered saline (PBS), $\mathrm{pH} \mathrm{6.5,} \mathrm{to} \mathrm{an} \mathrm{OD}_{600}$ of approximately 500 , divided in $40 \mu \mathrm{L}$ aliquots, and kept on ice.

Aliquots were incubated for $5 \mathrm{~min}$ at $30{ }^{\circ} \mathrm{C}$ in a heat block with vigorous shaking before uptake assay was started. To start the reaction, $10 \mu \mathrm{L}$ of a 5 times concentrated D-[1- $\left.{ }^{14} \mathrm{C}\right]$-xylose or D-[U- $\left.{ }^{14} \mathrm{C}\right]$-glucose solution, (Campro Scientific) was added. After exactly 20 seconds the reaction was stopped by the addition of $1 \mathrm{~mL}$ of appropriate ice-cold quenching buffer (100 mM PBS, pH 6.5, with $500 \mathrm{mM}$ unlabelled D-xylose or D-glucose), followed by vacuum filtration ( 0.45 $\mu \mathrm{m}$ HV filters, 1225 sampling manifold, Millipore), and two subsequent washing steps with $5 \mathrm{~mL}$ of ice-cold quenching buffer. After $5 \mathrm{~min}$ of drying in the vacuum manifold, the filters were transferred to scintillation vials with $4 \mathrm{~mL}$ scintillation liquid (Ultima Gold, Perkin Elmer), and activity was counted (Packard Tricarb 1600TR). All reactions were performed in triplicates. All values were corrected using triplicate negative control measurements without incubation, where the quenching solution was added prior to the addition of labeled substrate. Uptake rates at two typical substrate concentration ranges were measured; 1 to $100 \mu \mathrm{M}$ and 0.1 to $40 \mathrm{mM}$. Substrate solutions with an activity of approximately 5 to 5 ’000 Bq $\mu \mathrm{L}^{-1}$ were used. To determine kinetic parameters $\mathrm{K}_{\mathrm{m}}$ and $\mathrm{V}_{\max }$, the 
data was fitted to the Michaelis-Menten model using the least squares method

$$
V=\frac{V_{\max } \cdot[S]}{K_{m}+[S]}
$$

\subsubsection{Transcriptional analysis of $A$. niger and T. reesei genes}

RNA isolation from A. niger (Sloothaak et al., 2015) and T. reesei (Derntl et al., 2013) mycelium was done as described previously . Reverse transcription, quantitative PCRs and calculations were performed following the protocols and instruments described in Mach-Aigner et al., 2012 (Mach-Aigner et al., 2012). Primer sequences are provided in Additional file 9. Cycling conditions and control reactions were performed as described previously (Steiger et al., 2010). Normalization of the expression data was done using the previously described histone-like gene "hist" transcript (gene ID 207921) (Mach-Aigner et al., 2012) for A. niger, and genes sar1 and act1 for T. reesei (Steiger et al., 2010).

\subsection{Acknowledgements}

We would like to thank Dr Eckhard Boles for providing us the $S$. cerevisiae strain EBY.VW4000 and the plasmid p426HXT7-6His, Dr Ronald Hector for providing us the plasmid pRH315, Dr Monika Schmoll for providing us the yeast-mediated cloning protocol and Nikoleta Skarpa for her contribution to this work.

\section{Supplementary information}

Supplementary files for this chapter can be found online at:

https://biotechnologyforbiofuels.biomedcentral.com/articles/10.1186/s13068-0160564-4 


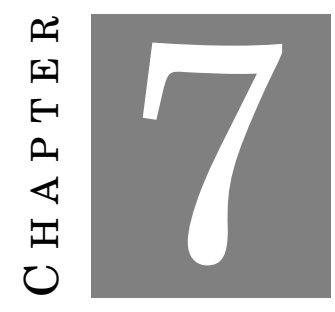

IDENTIFICATION OF A NOVEL L-RHAMNOSE UPTAKE TRANSPORTER IN THE FILAMENTOUS FUNGUS Aspergillus niger

\section{This chapter is adapted from:}

Sloothaak J, Odoni DI, Martins dos Santos VAP, Schaap PJ, Tamayo-Ramos JA. (2016). Identification of a Novel L-rhamnose Uptake Transporter in the Filamentous Fungus Aspergillus niger. In PLoS Genetics

(http://journals.plos.org/plosgenetics/article?id=10.1371/journal.pgen.1006468). 


\subsection{Abstract}

The study of plant biomass utilization by fungi is a research field of great interest due to its many implications in ecology, agriculture and biotechnology. Most of the efforts done to increase the understanding of the use of plant cell walls by fungi have been focused on the degradation of cellulose and hemicellulose, and transport and metabolism of their constituent monosaccharides. Pectin is another important constituent of plant cell walls, but has received less attention. In relation to the uptake of pectic building blocks, fungal transporters for the uptake of galacturonic acid recently have been reported in Aspergillus niger and Neurospora crassa. However, not a single L-rhamnose (6-deoxy-L-mannose) transporter has been identified yet in fungi or in other eukaryotic organisms. L-rhamnose is a deoxy-sugar present in plant cell wall pectic polysaccharides (mainly rhamnogalacturonan I and rhamnogalacturonan II), but is also found in diverse plant secondary metabolites (e.g. anthocyanins, flavonoids and triterpenoids), in the green seaweed sulfated polysaccharide ulvan, and in glycan structures from viruses and bacteria. Here, a comparative plasmalemma proteomic analysis was used to identify candidate L-rhamnose transporters in A. niger. Further analysis was focused on protein ID 1119135 (RhtA) (JGI A. niger ATCC 1015 genome database). RhtA was classified as a Family 7 Fucose: $\mathrm{H}^{+}$Symporter (FHS) within the Major Facilitator Superfamily. Family 7 currently includes exclusively bacterial transporters able to use different sugars. Strong indications for its role in L-rhamnose transport were obtained by functional complementation of the Saccharomyces cerevisiae EBY.VW.4000 strain in growth studies with a range of potential substrates. Biochemical analysis using L- $\left[{ }^{3} \mathrm{H}(\mathrm{G})\right]$-rhamnose confirmed that RhtA is a L-rhamnose transporter. The $r h t A$ gene is located in tandem with a hypothetical alpha-L-rhamnosidase gene ( $r h a B)$. Transcriptional analysis of $r h t A$ and $r h a B$ confirmed that both genes have a coordinated expression, being strongly and specifically induced by L-rhamnose, and controlled by RhaR, a transcriptional regulator involved in the release and catabolism of the methyl-pentose. RhtA is the first eukaryotic L-rhamnose transporter identified and functionally validated to date. 


\subsection{Introduction}

Organic carbon utilization by fungi is a biological process of great interest with many implications in ecology, agriculture and biotechnology. Their ubiquity and their ability to mobilize and metabolize a large variety of nutrients make fungi crucial players in the biogeochemical cycling of carbon in nature, in mutualistic symbiotic relationships with plants, and in pathogenic processes. Their physiological resources for carbon utilization and biotransformation have also enhanced their relevance in the fields of (food) fermentation, bioindustrial chemistry and pharmacy, as they can be exploited for the production of enzymes, chemicals and other components of interest. Thus, important efforts for the understanding of carbon utilization by fungi have been done, historically on those organisms that are genetically amenable, or have a direct impact on human affairs. In this sense, filamentous fungi from the Aspergillus genera, which include model species, species relevant for industrial applications, and human, animal and plant pathogens, have been deeply studied. In particular, the fungus Aspergillus niger has a versatile system for the degradation of the major plant cell wall polysaccharides: cellulose, hemicellulose and pectin, and due to its high enzyme secretory capacity is one of the main industrial producers of commercial enzymes for plant biomass conversion (Andersen et al., 2011; de Vries and Visser, 2001). A. niger is able to synthetize an abundance of extracellular enzymes for lignocellulose depolymerization, and the encoding genes as well as the regulatory circuits that control their expression have been identified and characterized in detail (Battaglia et al., 2011; de Oliveira et al., 2010; de Souza et al., 2013; van Peij et al., 1998). Pectin utilization by fungi has received less attention, probably due to its structural complexity and to the nature of its polysaccharides. Pectin is composed of mainly D-galacturonic acid (approx. $65 \%$ ), L-rhamnose, and branched with heterogeneous oligosaccharides (Mohnen, 2008). L-rhamnose is specifically found to be enriched in the pectic polysaccharide fractions rhamnogalacturonan I and II.

The pectin depolymerization enzyme network has been partly identified and characterized in several fungi (Martens-Uzunova and Schaap, 2009; Niu et al., 2015; Tamayo-Ramos et al., 2012), the catabolic pathways for the conversion of D-galacturonic acid and L-rhamnose have been genetically and biochemically characterized (Hilditch et al., 2007; Koivistoinen et al., 2012; Martens-Uzunova and Schaap, 2008; Zhang et al., 2011), and recently the transcription factors responsible for L-rhamnose and D-galacturonic acid utilization, RhaR and GaaR, have been identified (Alazi et al., 2016; Gruben et al., 2014). Regarding the 
uptake of the specific pectin components, transporters responsible for the uptake of D-galacturonic acid have been reported in A. niger and Neurospora crassa (Benz et al., 2014; Sloothaak et al., 2014). However, not a single L-rhamnose transporter has been identified in fungi, or in any other eukaryotic organism. Besides its structural role in pectin, L-rhamnose is part of plant glycoproteins and secondary metabolites too, it is an important component of the green seaweed sulfated polysaccharide ulvan, and it is also present in glycan structures from virus and bacteria (Lahaye and Robic, 2007). In prokaryotes, L-rhamnose uptake occurs via transporters from the RhaT family (2.A.7.6), which belongs to the drug/metabolite transporter superfamily (2.A.7) (Jack et al., 2001), but eukaryotic transporters belonging to the RhaT family have not been reported. Recently, an intracellular UDP-rhamnose/UDP-galactose transporter (nucleotide sugar transporter; NST), also member of the DMT superfamily, and located on the Golgi apparatus of Arabidopsis thaliana, was identified (Rautengarten et al., 2014). However NSTs are involved in transport processes related to the biosynthesis of plant cell wall components, and glycan structures, not found in eukaryotic plasma membranes, and not related to the uptake of sugars present in the environment. Monosaccharide transport by fungi has been investigated mainly in Saccharomyces cerevisiae in which transport of simple sugars is mediated only through facilitated diffusion by transporters from the sugar porter family, the largest within the major facilitator superfamily (MFS) (Leandro et al., 2009). The use of yeast monosaccharide transporter null mutants allowed for individual characterization of the individual transporters responsible for the uptake of $D$ glucose, D-fructose, D-mannose and D-galactose in yeast (Leandro et al., 2009; Reifenberger et al., 1997; Wieczorke et al., 1999). Yeast transporter null mutants were subsequently also used for the functional characterization of sugar porters from other fungal species (dos Reis et al., 2013; Du et al., 2010; Leandro et al., 2013; Polidori et al., 2007; Saloheimo et al., 2007; Vankuyk PA et al., 2004).

The analyses of the global transcriptomic and proteomic responses of fungi to a variety of specific culture conditions are useful approaches to get insights in the specific structural and regulatory elements required for the utilization of specific carbon sources. In order to identify L-rhamnose transporter candidates, in this study a comparative plasmalemma proteome analysis was performed. The identification and functional validation of a eukaryotic L-rhamnose transporter is reported for the first time. 


\subsection{Results}

\subsubsection{Comparative plasmalemma proteome analysis for the identification of $A$. niger L-rhamnose transporter candidates}

A recently developed approach for the study of the A. niger transportome through its plasma membrane proteomic analysis was used in order to perform a differential protein expression analysis of $A$. niger major facilitator superfamily (MFS) transporters. The approach is based on a LC-MS/MS analysis of plasmalemma enriched cellular fractions, and the method was applied to study the A. niger response to different D-glucose concentrations (Sloothaak et al., 2015). In the present study, a similar experimental set-up was used, but here the A. niger response to the presence of D-mannose, L-arabinose, D-xylose and L-rhamnose was studied. Mycelium of $A$. niger N400 was pre-grown for $18 \mathrm{~h}$ in minimal medium supplemented with $100 \mathrm{mM}$ D-sorbitol as sole carbon source and equal amounts of biomass was transferred to controlled fermenters containing minimal medium with the following carbon source compositions: D-sorbitol $100 \mathrm{mM}$ plus $0.1 \mathrm{mM}$ D-xylose, $5 \mathrm{mM}$ D-mannose, $5 \mathrm{mM}$ L-arabinose or $5 \mathrm{mM}$ L-rhamnose. The initial $\mathrm{pH}$ of these cultures was set at pH 4.0 and controlled at a lower limit of $\mathrm{pH} 3.5$. Two hours after inoculation, mycelium was taken and cellular fractions enriched for plasma membranes were obtained as previously reported (see Materials and Methods and (Sloothaak et al., 2015) for details). High-resolution analysis of the sugar content in the culture medium at the time of sampling showed that in all four conditions the respective sugars were being consumed (S1 Fig), so the presence of active transporters for the different monosaccharides could be expected.

For each culture condition peptide MS/MS spectra, obtained from the LCMS/MS analysis of the enriched plasmalemma fractions, were processed as described in the Materials and Methods section. In total, 958 proteins were identified, of which 510 were present in all four conditions, while 41, 40, 65, and 43 proteins were present exclusively in the L-arabinose, D-mannose, L-rhamnose, and D-xylose conditions, respectively (S1 Dataset). The aim of this study was to identify specific L-rhamnose transporters, so the comparative proteome analysis was focussed on the identification and abundance analysis of candidate sugar porters. A total of 15 MFS transporters were identified in the presence of D-xylose, 16 in the presence of D-mannose, 19 in the presence of L-arabinose and 21 in the presence of L-rhamnose, of which 8 were shown to be exclusively detected in the 
L-rhamnose condition (Table 7.1). From these eight, a subgroup of five having strain ATCC 1015 (Andersen et al., 2011) protein ID 1096151, 1119135, 1142034, 1147409 and 1156895 respectively were also absent in a previously generated dataset, where the $A$. niger plasmalemma proteome response to high and low concentrations of D-glucose was studied (Sloothaak et al., 2015). In summary, by analysing the $A$. niger plasma membrane proteome response to seven different carbon source compositions (D-sorbitol $100 \mathrm{mM}$, D-sorbitol $100 \mathrm{mM}$ plus Dglucose $1 \mathrm{mM}$, D-sorbitol $100 \mathrm{mM}$ plus D-glucose $60 \mathrm{mM}$, D-sorbitol $100 \mathrm{mM}$ plus D-xylose $0.1 \mathrm{mM}$, L-arabinose $5 \mathrm{mM}$, D-mannose $5 \mathrm{mM}$, and L-rhamnose $5 \mathrm{mM}$ ) five putative transport proteins could be identified that were present only in the presence of L-rhamnose, which strongly suggested involvement of one or more of these transporters in the uptake of L-rhamnose.

A detailed protein sequence analysis of these transporters and of their encoding genes highlighted some interesting features of protein ID 1119135, hereinafter referred to as RhtA for Rhamnose transporter A. Domain analysis of the transporters revealed that RhtA was exceptional because it possessed a L-fucose permease domain structure (FucP; COG0738/IPR005275). FucP domain transporters have been shown to be able to use different deoxy sugars as substrate, such as L-fucose, 2-deoxy-D-ribose and 2-deoxy-D-glucose, but also monosaccharides such as L-arabinose, D-galactose, D-glucose and D-mannose (Christensen et al., 2003; Gunn et al., 1994; Paulsen et al., 1998). To our knowledge transporters with a FucP domain structure have only been characterized from bacteria. However, the particular domain structure is well represented throughout the fungal kingdom. Through a Bi-directional Best Hit (BBH) Blast analysis, putative RhtA orthologs were inferred. Homologous sequences were found throughout different fungal orders, but only 5 BBH's were identified when analyzing the available genomes of Aspergillus spp. (S1 Table).

A domain based classification of RhtA, using amino acid sequences of 27 functionally validated fungal MFS sugar transporters, 2 bacterial L-rhamnose transporters, and 4 characterized bacterial FucP domain symporters as input, grouped the RhtA transporter with the latter group (Fig 7.1). These transporters belong to the Fucose:H1 symporter (FHS) family (TC 2.A.1.7) within the Major Facilitator Superfamily (Pao et al., 1998) (Fig 7.1). The domain architecture COG0738 is defined by 13 reference sequences, of which Bacillus subtilis GlcP, Escherichia coli FucP and Helicobacter pylori HP1174 (Gunn et al., 1994; Paulsen et al., 1998; Psakis et al., 2009) have been characterized, while DeoP from Salmonella enterica is probably a 2-deoxy-D-ribose permease (Christensen et al., 2003). E. coli FucP transports L-fucose, L-arabinose and D-galactose but it is not able to transport 
Table 7.1: Organelle specific differential expression enrichment analysis. n.d.: not detected; *: same protein group; ProtID underlined indicate putative transporters detected in L-rhamnose, but not in L-arabinose, D-mannose and D-xylose conditions. ProtID in bold indicate putative transporter proteins not detected as well in the previous proteomic analysis performed by Sloothaak et al. (2015), where A. niger was grown in the presence of D-sorbitol $100 \mathrm{mM}$, D-sorbitol $100 \mathrm{mM}$ plus D-glucose $1 \mathrm{mM}$, and D-sorbitol $100 \mathrm{mM}$ plus D-glucose $60 \mathrm{mM}$ (Sloothaak et al., 2015). ProtIDs were obtained from the JGI ATCC1015 database (Nordberg et al., 2013).

\begin{tabular}{|c|c|c|c|c|}
\hline \multirow[t]{2}{*}{ Prot ID } & \multicolumn{4}{|c|}{ Relative abundance $\pm \mathrm{sd}(\%) \times 100$} \\
\hline & L-arabinose & D-mannose & L-rhamnose & $\begin{array}{c}\text { D-sorbitol + } \\
\text { D-xylose }\end{array}$ \\
\hline 1089440 & n.d. & n.d. & $1.91 \pm 0.98$ & n.d. \\
\hline$\overline{\mathbf{1 0 9 6 1 5 1}}$ & n.d. & n.d. & $4.20 \pm 2.40$ & n.d. \\
\hline$\overline{1101809}$ & $5.12 \pm 1.73$ & $1.60 \pm 0.08$ & $7.94 \pm 0.90$ & $2.95 \pm 0.24$ \\
\hline 1105147 & $6.20 \pm 0.21$ & $8.13 \pm 0.82$ & $11.78 \pm 0.22$ & $6.78 \pm 0.44$ \\
\hline 1105500 & $0.54 \pm 0.20$ & n.d. & n.d. & n.d. \\
\hline 1111630 & $0.49 \pm 0.10$ & $1.71 \pm 0.15$ & $1.31 \pm 0.14$ & $1.38 \pm 0.45$ \\
\hline$\frac{\text { 1119135 }}{\text { (RhtA) }}$ & n.d. & n.d. & $10.94 \pm 4.21$ & n.d. \\
\hline$\overline{1121621}$ & $3.64 \pm 1.31$ & $5.90 \pm 0.73$ & n.d. & $4.96 \pm 0.48$ \\
\hline 1122202 & $0.63 \pm 0.20$ & $0.92 \pm 0.05$ & $0.46 \pm 0.19$ & $0.28 \pm 0.01$ \\
\hline 1125086 & $37.93 \pm 5.96$ & n.d. & n.d. & n.d. \\
\hline 1128338 & n.d. & $4.98 \pm 1.11$ & n.d. & $2.03 \pm 0.07$ \\
\hline 1129336 & n.d. & $0.39 \pm 0.08$ & n.d. & $0.26 \pm 0.01$ \\
\hline 1142034 & n.d. & n.d. & $2.26 \pm 0.04$ & n.d. \\
\hline$\overline{1142882}$ & $2.95 \pm 0.32$ & $2.30 \pm 0.56$ & $4.40 \pm 0.11$ & $2.21 \pm 0.50$ \\
\hline 1143191 & n.d. & n.d. & $7.04 \pm 0.97$ & n.d. \\
\hline$\overline{1143598}$ & $3.34 \pm 0.89$ & $4.59 \pm 2.03$ & $6.51 \pm 5.28$ & $8.51 \pm 0.12$ \\
\hline 1144375 & $5.80 \pm 2.89$ & $0.72 \pm 0.23$ & $15.25 \pm 3.84$ & n.d. \\
\hline 1144791 & $16.06 \pm 4.06$ & n.d. & $17.75 \pm 2.24$ & $6.20 \pm 1.05$ \\
\hline 1147409 & n.d. & n.d. & $0.78 \pm 0.44$ & n.d. \\
\hline$\overline{\mathbf{1 1 5 6 8 9 5}}$ & n.d. & n.d. & $10.92 \pm 0.57$ & n.d. \\
\hline$\overline{1160647}$ & $2.47 \pm 1.61$ & n.d. & n.d. & n.d. \\
\hline $\begin{array}{l}1164538 \\
1188786^{*}\end{array}$ & $0.55 \pm 0.11$ & $1.30 \pm 0.12$ & $1.03 \pm 0.24$ & $0.95 \pm 0.01$ \\
\hline 1165706 & n.d. & n.d. & n.d. & $0.06 \pm 0.05$ \\
\hline 1167504 & $1.07 \pm 0.09$ & $0.39 \pm 0.12$ & n.d. & n.d. \\
\hline 1169204 & $0.54 \pm 0.13$ & n.d. & n.d. & $5.07 \pm 0.95$ \\
\hline 1178623 & $7.53 \pm 2.54$ & $5.20 \pm 0.00$ & $5.64 \pm 4.12$ & n.d. \\
\hline 1180703 & n.d. & n.d. & $2.61 \pm 0.25$ & n.d. \\
\hline$\overline{1188093}$ & $0.85 \pm 0.08$ & $0.96 \pm 0.15$ & $1.59 \pm 0.15$ & $0.78 \pm 0.16$ \\
\hline 1188840 & n.d. & $0.60 \pm 0.24$ & n.d. & n.d. \\
\hline 1189214 & $35.93 \pm 0.54$ & n.d. & $2.87 \pm 1.02$ & n.d. \\
\hline
\end{tabular}

L-rhamnose, B. subtilis GlcP has high affinity for D-glucose and D-mannose, and H. pylori HP1174 is able to use D-glucose, D-galactose, D-mannose and 2-deoxy-Dglucose as substrates. L-fucose and L-rhamnose are structurally related, as both of them are methyl pentoses, however there have been no reports on the ability of FHS symporters to use L-rhamnose as substrate, for which specific transporters from the L-rhamnose proton symport family (RhaT; cl05728/IPR004673) have 
been described in bacteria (Tate et al., 1992). Characterized bacterial L-rhamnose transporters from the L-rhamnose transporter family (RhaT) (TC 2.A.7.6) clustered together in a separate subgroup in the phylogenetic tree (Fig 1). This was expected because they are not related to the MFS (2.A.1) and have no similarities with proteins from this superfamily (Saier, 2000). The remaining fungal functionally validated sugar transporters are clustered in two additional subgroups corresponding to other MFS families: the sugar porter (SP) family (TC 2.A.1.1) and the drug:H1 antiporter-1 (12 spanner) (DHA1) (TC 2.A.1.2) (Fig 7.1). 


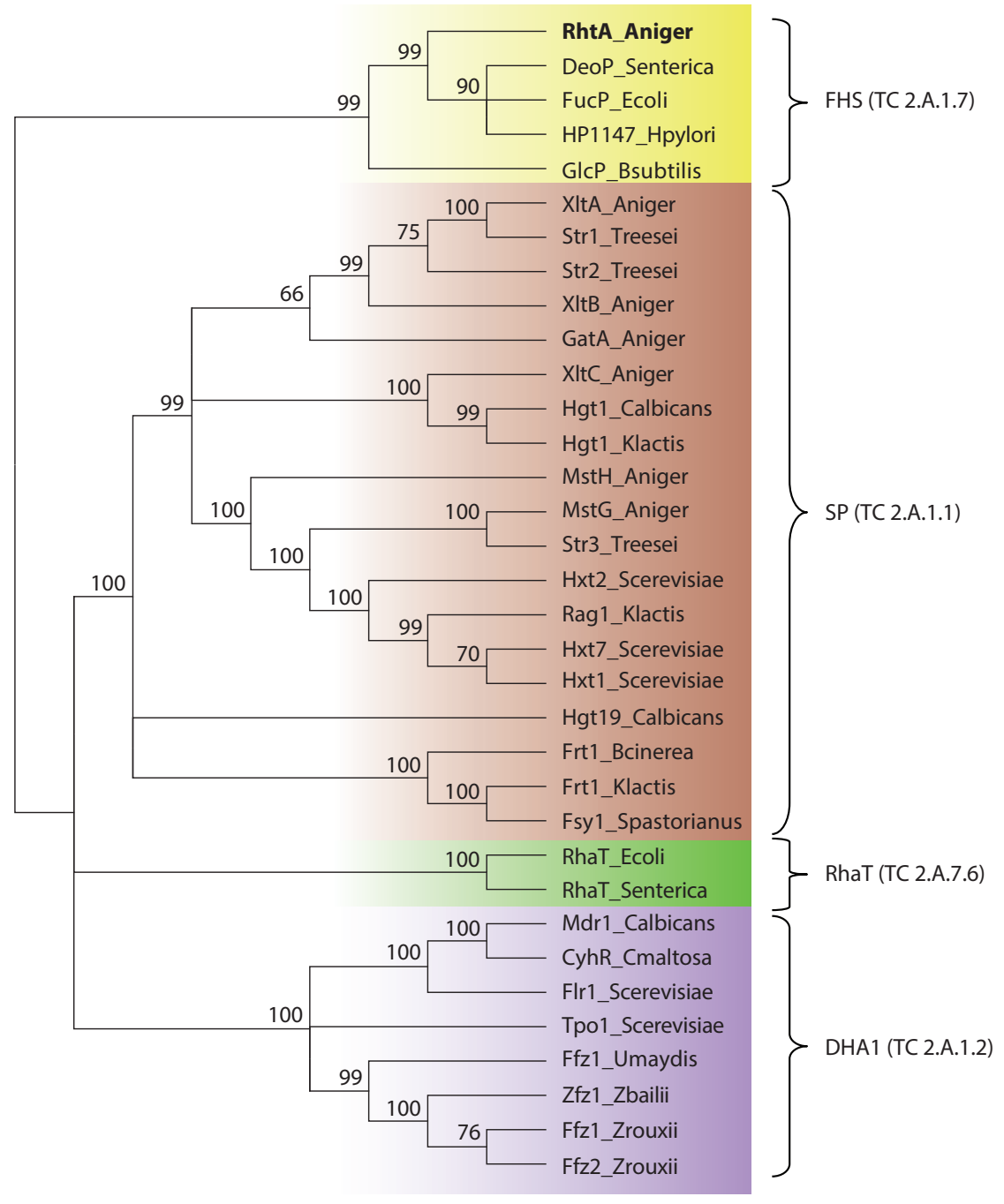

FIGURE 7.1. Classification of A. niger RhtA. Sequences of biochemically characterized sugar transporters were collected and a multiple sequence alignment was created using PRALINE alignment suite, which takes secondary structure predictions into account (Simossis and Heringa, 2005). A neighbour-joining tree was then generated with 1'000 bootstrap replicates.

Analysis of the genomic locus of the RhtA encoding gene revealed directly upstream of $r h t A$ an ORF coding for a hypothetical secreted glycoside hydrolase, 
hereinafter referred to $r h a B$, from the GH78 family (Protein ID 131668) which only includes $\alpha$-L-rhamnosidases. The hypothetical RhaB is a 832 amino acid protein with low similarity to functionally validated $\alpha$-L-rhamnosidases. However, its amino acid number is in the range of RhaE from A. nidulans (861 aa), and many of the characterized bacterial rhamnosidases (Tamayo-Ramos et al., 2012). The $r h a B$-rhtA tandem localization, which could conform a L-rhamnose uptake system in A. niger, was found to be present as well in Aspergillus luchuensis and Aspergillus kawachii, which have been reported to be the same species (Hong et al., 2013), and are closely related to A. niger.

Taking the above-described findings into account, RhtA was considered a strong candidate to be a transporter specific for L-rhamnose. In order to validate this hypothesis, and to unravel the function of this eukaryotic transporter with a FucP domain signature, RhtA was selected for functional validation and characterisation in the present study.

\subsubsection{Functional validation of the $A$. niger sugar transporter RhtA}

In order to test the functionality of the RhtA transporter, the engineered $S$. cerevisiae strain EBY.VW.4000, a monosaccharide transporter null mutant, was chosen as host for the heterologous expression of the rhtA coding gene. S. cerevisiae is not able to use L-rhamnose as a carbon source, so a direct functional complementation approach based on the use of this deoxy sugar could not be performed with this strain. Despite this, and as discussed below, heterologous expression of the transporter in this genetic background gave clear insights about the possible role of RhtA on L-rhamnose transport.

The yeast strain was transformed with the $2 \mu$ expression plasmid p426HXT76 His-rhtA, containing the gene's cDNA under control of the constitutive promoter HXT7p and the terminator CYC1t. Single colony transformants were isolated from minimal medium agar plates containing $2 \%(\mathrm{w} / \mathrm{v})$ maltose and the ability of $r h t A$ to restore growth of the EBY.VW.4000 transformant strain in the presence of different monosaccharides was studied. Ten-fold serial dilutions of logarithmically growing cells from at least three different transformants expressing $r h t A$ were spotted on different minimal medium plates supplemented with $1 \%(\mathrm{w} / \mathrm{v})$ of the following carbon sources: D-glucose (G; $56 \mathrm{mM})$, D-fructose (F; $56 \mathrm{mM})$, D-mannose (Mn; $56 \mathrm{mM})$ and maltose $(\mathrm{M} ; 29 \mathrm{mM})$. Yeast rhtA transformants showed an ability to restore growth on D-fructose, albeit at a slow pace, but were not able to restore growth on D-glucose and D-mannose (Fig 7.2). This 
result indicated that RhtA was functional as a transporter in $S$. cerevisiae, but as expected none of the substrates tested seemed to be ideal for this transporter. The fact that D-fructose was used as a substrate by RhtA allowed us to perform sugar competition assays on plate, which gave more insights in possible additional substrates for this transporter. Hence, the ability of an $r h t A$ transformant to grow in the presence of $\mathrm{D}$-fructose $(\mathrm{F} ; 28 \mathrm{mM})$ was compared to its ability to grow in plates containing D-fructose $(\mathrm{F} ; 28 \mathrm{mM})$ mixed with either D-glucose $(\mathrm{G} ; 56$ $\mathrm{mM}$ or $5.6 \mathrm{mM})$, D-xylose (X; $66 \mathrm{mM}$ or $6.6 \mathrm{mM})$, L-arabinose (A; $66 \mathrm{mM}$ or 6.6 $\mathrm{mM})$, D-sorbitol (S; $55 \mathrm{mM}$ or $5.5 \mathrm{mM})$ or L-rhamnose (R; $61 \mathrm{mM}$ or $6.1 \mathrm{mM})$. The $r h t A$ transformant strain was able to grow in the presence of most of the sugar mixes tested, but it was unable to grow on $\mathrm{D}$-fructose in the presence of a high and low concentration of L-rhamnose, suggesting that D-fructose uptake by RhtA was strongly inhibited by L-rhamnose (Fig 7.3a). D-fructose uptake by RhtA was also inhibited by L-arabinose, at a concentration of $66 \mathrm{mM}$, but not at a concentration of $6.6 \mathrm{mM}$. The results suggested that RhtA could have a higher affinity for L-rhamnose than for any of the other sugars tested. To determine the lower boundary for D-fructose uptake inhibition, the experiment was then repeated with lower L-rhamnose concentrations (0.0006 $\mathrm{mM}$ to $6.1 \mathrm{mM})$ in the presence of D-fructose ( $28 \mathrm{mM}$ ) as carbon source (Fig 7.3b). As shown in Fig 7.3b, growth was inhibited by L-rhamnose concentrations as low as $0.06 \mathrm{mM}$. The fact that such a low concentration of L-rhamnose, around 500 times lower than of D-fructose, was able to inhibit growth, pointed to the deoxy sugar as a possible true substrate of the RhtA transporter. To prove that the strong growth inhibitory capacity of L-rhamnose was exclusively associated to the RhtA transporter, and not due to an unexpected toxicity of L-rhamnose on the yeast itself, a different strain (CEN.PK2-1C), isogenic to EBY.VW.4000, harboring the p426HXT7-6HisrhtA, was also grown in the same conditions. The CEN.PK2-1C strain expressing RhtA was able to grow in the presence of D-fructose $28 \mathrm{mM}+$ L-rhamnose 0.06 $\mathrm{mM}$ (Fig 7.4a), thus indicating that the D-fructose uptake inhibition invoked by L-rhamnose was RhtA dependent, as inhibition of growth did not occur in the yeast strain with functional endogenous D-fructose transport systems.

Finally, a competition plate assay was performed using two alternative deoxy sugars sugars: L-fucose (Fc; $6.1 \mathrm{mM}$ ) and 2-deoxy-D-ribose (Dr; $7.5 \mathrm{mM}$ ). As it can be observed in (Fig 7.4b) the RhtA transformant showed normal growth. Taken together, these results suggested that $A$. niger RhtA could be a very specific transporter for L-rhamnose, unable to use other deoxy sugars as substrates. 


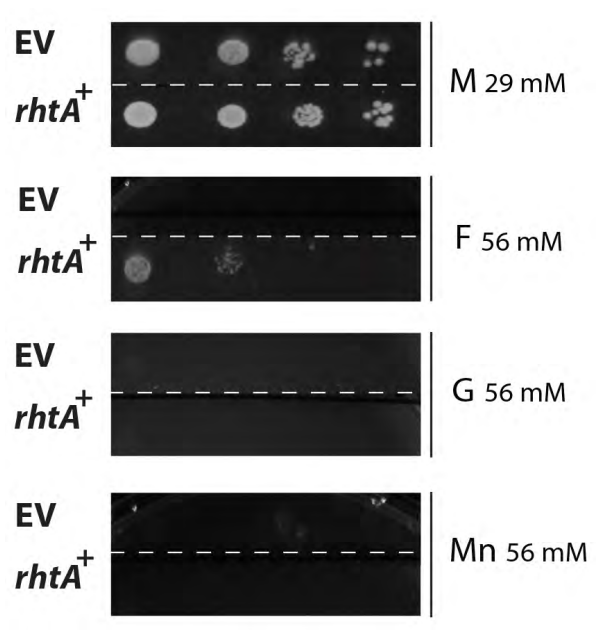

FIGURE 7.2. RhtA functional analysis in yeast. Growth of strain EBY.VW4000 expressing the $r h t A$ gene $\left(r h t A^{+}\right)$or harbouring the empty expression vector p426HXT7-6His (EV) in minimal medium agar plates containing maltose (M; $29 \mathrm{mM})$, D-fructose (F; $56 \mathrm{mM})$, D-glucose (G; $56 \mathrm{mM})$ or D-mannose $(\mathrm{Mn} ; 56 \mathrm{mM})$ as sole carbon sources. Agar plates were incubated at $30^{\circ} \mathrm{C}$ for $96 \mathrm{~h}$. Transformants expressing RhtA showed the same growth pattern; the figure depicts only one representative transformant.

\subsubsection{Transcriptional analysis of the A. niger sugar transporter coding gene $r h t A$}

According to the RhtA protein abundance profile obtained by plasmalemma proteome analysis, the expression of the transporter seemed to be specifically induced by L-rhamnose. This fact, together with the additional findings described above, indicated that RhtA could be a L-rhamnose transporter. However, in yeast complementation experiments RhtA showed an ability to transport D-fructose, so in $A$. niger the transporter's biological role could be related to D-fructose uptake as well. Having this into account, new fermentations of the A. niger $\mathrm{N} 400$ wild type strain were performed, using the same set-up as described above, in order to study the transcriptional response of $r h t A$ to D-sorbitol $100 \mathrm{mM}$ (reference), L-rhamnose $5 \mathrm{mM}$, and D-fructose $5 \mathrm{mM}$. Samples, obtained two hours after mycelium transfer, were processed, and RT-qPCR analysis was performed. As expected, the $r h t A$ gene was strongly induced in the presence of L-rhamnose, while its expression levels in the presence of $\mathrm{D}$-fructose were found to be similar to those observed in the reference condition (Fig 7.5). This result showed that L-rhamnose, but not 
a)

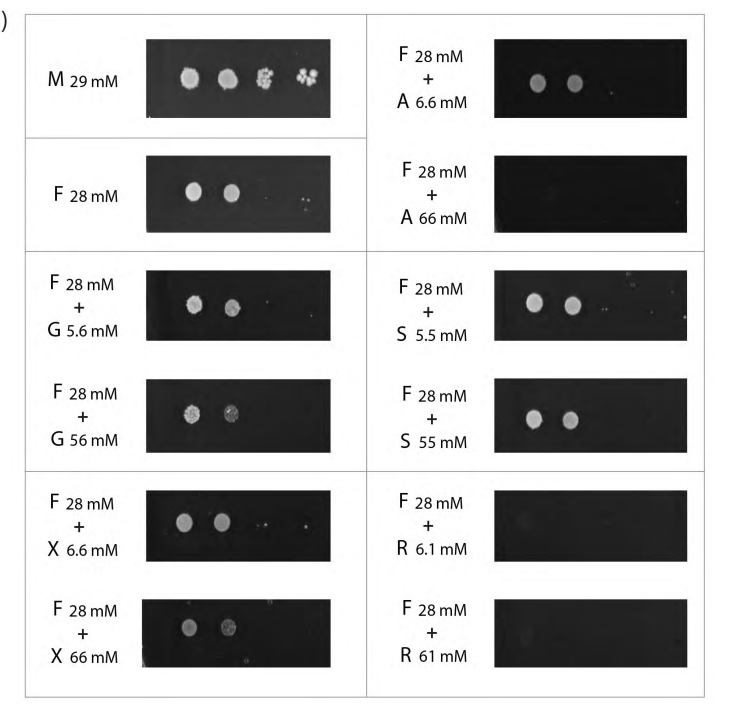

b)

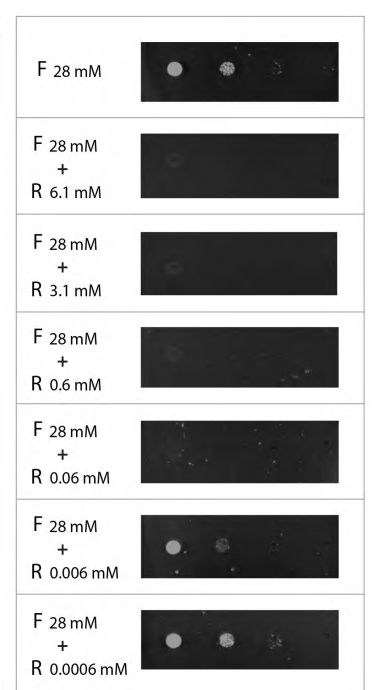

FIgURE 7.3. EBY.VW4000 $\boldsymbol{r h a A}^{+}$growth inhibition assays. a) Growth of yeast strain EBY.VW4000 expressing the $r h t A$ gene on minimal medium with maltose $(\mathrm{M} ; 29 \mathrm{mM})$, D-fructose $(\mathrm{F} ; 28 \mathrm{mM})$, and D-fructose (F; $28 \mathrm{mM})$ supplemented with the potentially competing carbon sources: D-glucose (G; $56 \mathrm{mM}$ and 5.6 mM), D-xylose (X; $66 \mathrm{mM}$ and $6.6 \mathrm{mM}$ ), L-arabinose (A; $66 \mathrm{mM}$ and $6.6 \mathrm{mM}), \mathrm{D}$ sorbitol (S; $55 \mathrm{mM}$ and $5.5 \mathrm{mM})$ or L-rhamnose (F; $61 \mathrm{mM}$ and $6.1 \mathrm{mM})$; b) growth of yeast strain EBY.VW4000 expressing the $r h t A$ gene on minimal medium with D-fructose $(\mathrm{F} ; 28 \mathrm{mM})$, and $\mathrm{D}$-fructose $(\mathrm{F} ; 28 \mathrm{mM})$ supplemented with a range of L-rhamnose concentrations $(0.0006 \mathrm{mM}$ to $6.1 \mathrm{mM})$.

D-fructose, acts as an inducer of the RhtA transporter at transcriptional level, suggesting that RhtA is not a natural D-fructose transporter.

As mentioned above, the gene $r h a B$, coding for a hypothetical $\alpha$-L-rhamnosidase (JGI A. niger ATCC 1015 Protein ID 131668), was found to be located directly upstream of $r h t A$. The amino acid analysis of the hypothetical $\alpha$-L-rhamnosidase revealed the presence of a classical $\mathrm{N}$-terminal secretion signal peptide. Thus, it would be possible that both $r h a B$ and $r h t A$ gene products have a coordinated action, releasing and transporting L-rhamnose monomers respectively. This would imply that both proteins are coordinately expressed in the presence of the sugar. To obtain more insight into the transcriptional responses of $r h t A$ and $r h a B$ to L-rhamnose, time course fermentations were done, in cultures containing either D-sorbitol $100 \mathrm{mM}$, L-rhamnose $1 \mathrm{mM}$ or L-rhamnose $5 \mathrm{mM}$ as sole carbon 

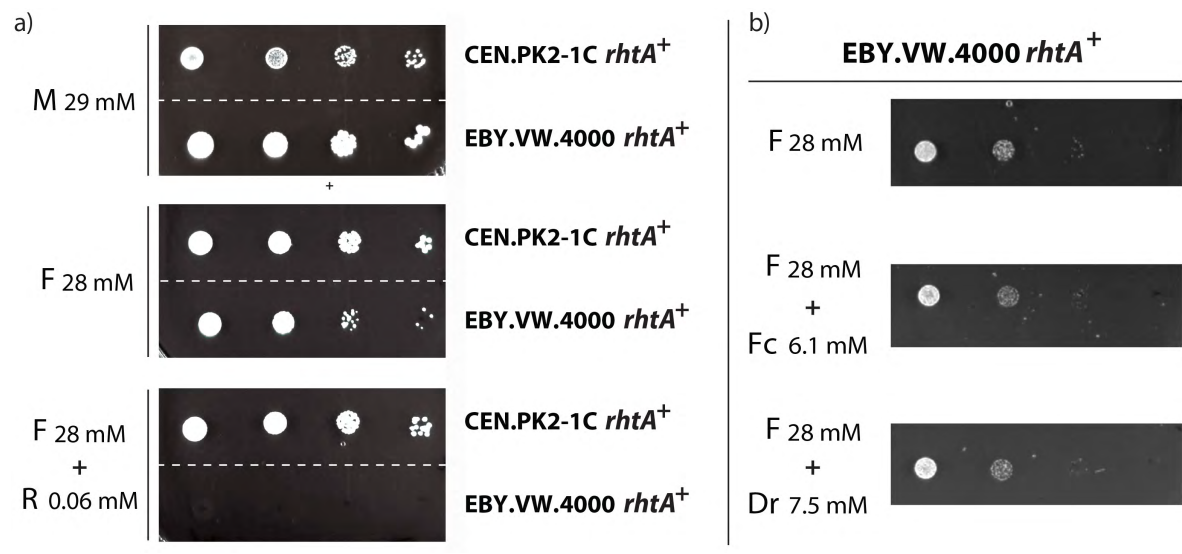

\section{FIGURE 7.4. EBY.VW4000 $\boldsymbol{r h a A}^{+}$and CEN.PK2-1C $\boldsymbol{r h a A}^{+}$growth inhibition assays. a) Growth of yeast strains CEN.PK2-1C and EBY.VW4000 expressing the $r h t A$ gene on minimal medium with maltose (M; $29 \mathrm{mM})$, D-fructose $(\mathrm{F} ; 28 \mathrm{mM})$ or D-fructose (F; 28 $\mathrm{mM}$ ) supplemented with L-rhamnose (R; $0.06 \mathrm{mM})$; b) Growth of EBY.VW4000 rhaA ${ }^{+}$on minimal medium containing D-fructose (F; $28 \mathrm{mM}$ ) or D-fructose (F; $28 \mathrm{mM})$ supplemented with L-fucose (Fc; $6.1 \mathrm{mM}$ ) or 2-deoxy-D-ribose (Dr; $6.1 \mathrm{mM})$.}

source. Sampling was performed every hour after mycelium transfer, until the L-rhamnose was depleted from the medium for two hours. The RT-qPCR results obtained showed a fast transcriptional response of $r h t A$ to the presence of Lrhamnose (Fig 7.6), while in the D-sorbitol reference condition $r h t A$ expression remained at low level (S2 Fig). One hour after mycelium transfer the $r h t A$ expression level in the presence of an initial L-rhamnose concentration of $1 \mathrm{mM}$ was approximately 1'000 fold higher than those observed in the reference condition (D-sorbitol $100 \mathrm{mM}$; $\mathrm{t}=1 \mathrm{~h}$ ). A maximum was observed two hours after transfer, with $r h t A$ transcriptional levels being 5'000 fold higher than in the reference condition. The rhtA transcription profile for the first three hours was the same in the $5 \mathrm{mM}$ L-rhamnose fermentation (S2 Fig). The maximum rhtA expression levels remained constant during the time course experiment until the deoxy sugar was completely consumed (Fig 7.6). After this point, rhtA mRNA levels decreased approximately 80 fold. Once the $r h t A$ mRNA levels decreased, they remained constant at least 2 hours, being still around 50 times higher than its expression levels in the reference condition. High mRNA stability could be the reason why the $r h t A$ expression levels kept being relatively high, even several hours after the sugar was completely consumed. 


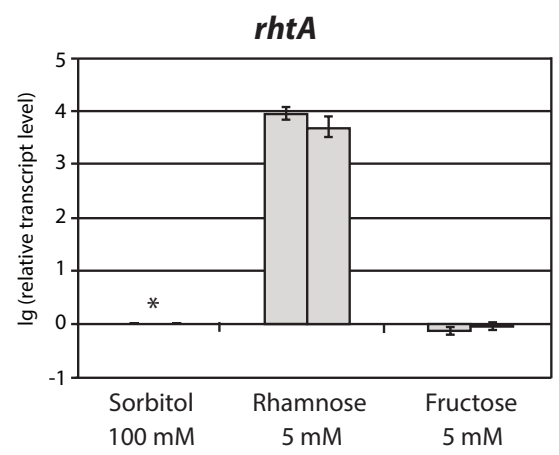

FIGURE 7.5. Transcriptional analysis of $\boldsymbol{r h t A}$. Relative transcription levels were measured by RT-qPCR, in A. niger N400 sampled 2 hours after mycelium transfer to minimal medium with $100 \mathrm{mM}$ D-sorbitol (reference), $5 \mathrm{mM}$ L-rhamnose or $5 \mathrm{mM}$ D-fructose. Transcript levels are relative to reference sample (D-sorbitol $100 \mathrm{mM}$ ), indicated with an asterisk. Results are given as relative transcript ratios in logarithmic scale $(\lg (10))$. The values provided in the figures correspond to two biological replicates per culture condition. Error bars are means of three technical replicates.

The results displayed in Fig 7.6 show that $r h a B$ has a similar expression profile to the one observed for $r h t A$ : its expression was activated in the presence of L-rhamnose and remained constant until the sugar was depleted from the medium (reference condition: D-sorbitol $100 \mathrm{mM}$; $\mathrm{t}=1 \mathrm{~h}$ ). Therefore both genes seem to have a specific coordinated response to the presence of L-rhamnose in the environment.

To further understand the regulatory mechanisms underlying the L-rhamnose uptake system encoded by the $r h t A$ and $r h a B$ genes, the role of the regulators RhaR, involved in L-rhamnose release and catabolism (Gruben et al., 2014), and CreA, mediating carbon catabolite repression in plant cell wall utilisation systems (de Vries et al., 1999), was studied. To do this, a transcriptional analysis of both genes in the strains N402 (WT), NW283 ( $\Delta c r e A)$ and JS14 ( $\Delta r h a R$ ) (see Materials and Methods section for construction details) was done. The wild type and the $\triangle c r e A$ and $\triangle r h a R$ strains were pre-cultured in MM with $100 \mathrm{mM}$ Dsorbitol for $18 \mathrm{~h}$, and transferred to $\mathrm{MM}$ with either $5 \mathrm{mM}$ L-rhamnose or 5 mM L-rhamnose plus $50 \mathrm{mM}$ D-glucose. Samples were taken two hours after mycelium transfer, and were subsequently processed for RT-qPCR analysis. As previously observed, both $r h t A$ and $r h a B$ were induced by L-rhamnose. Their transcriptional levels in the presence of the deoxy sugar were similar in both 


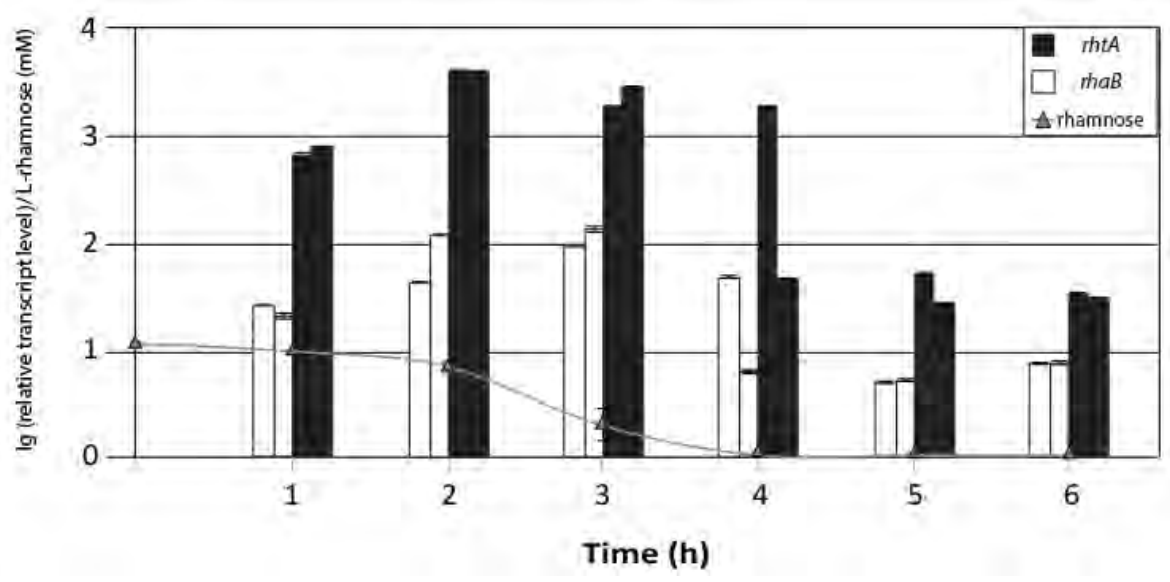

FIGURE 7.6. Time course transcriptional analysis of rhtA and rhaB. Relative transcription levels, measured by RT-qPCR, of $r h t A$ (black bars) and rhaB (white bars) during A. niger N400 fermentations in minimal medium with an initial concentration of Lrhamnose $1 \mathrm{mM}$. Concentration of L-rhamnose over time is represented by grey line with triangles (concentration at $\mathrm{t}=4 \mathrm{~h}$ is equal to 0 ). Transcript levels of both genes always refer to the reference sample (D-sorbitol $100 \mathrm{mM} ; \mathrm{t}=1 \mathrm{~h})$. Results are given as relative transcript ratios in logarithmic scale $(\lg (10))$. The values provided in the figures correspond to two biological replicates per culture condition. Error bars are means of three technical replicates.

the wild type (N402) and $\alpha c r e A$ (NW283) strains (Fig 7.7). In the presence of L-rhamnose plus D-glucose, $r h t A$ and $r h a B$ were heavily repressed in the wild type strain, and only partly derepressed in the $\triangle c r e A$ mutant. Regarding the regulatory mechanisms mediating the activation of $r h t A$ and $r h a B$, the expression of both genes in the presence of L-rhamnose was strongly reduced in the $\Delta r h a R$ strain, suggesting that the RhaR transcriptional activator is responsible for the induction of these genes.

\subsubsection{Determination of the RhtA ability to transport L-rhamnose}

To prove that RhtA can use L-rhamnose as substrate, a tritium labeled L-[3H(G)]rhamnose uptake experiment was performed. In this experiment, the L-rhamnose uptake ability of the EBY.VW4000_RhtA strain was determined, using as a negative control the EBY.VW4000 strain expressing the A. niger specific D-xylose 

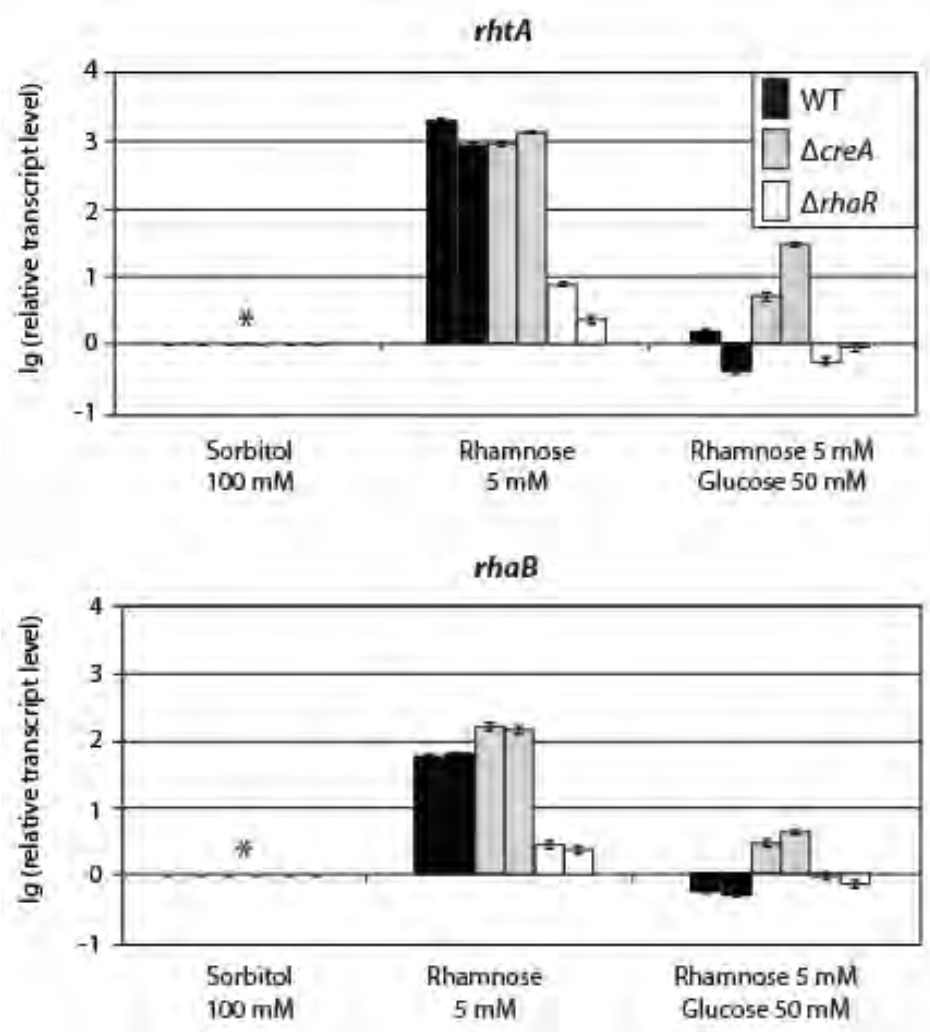

FIGURE 7.7. Role of the CreA and RhaR transcriptional regulators on the expression of $\boldsymbol{r h t A}$ and rhaB. Strains N402 (WT; black bars), NW283 ( $\triangle c r e A$; grey bars), and JS014 ( $\Delta r h a R$; open bars) were used. Relative transcription levels were measured by RTqPCR in samples obtained 2 hours after mycelium transfer to $5 \mathrm{mM}$ L-rhamnose or 5mM L-rhamnose plus $50 \mathrm{mM}$ D-glucose. Relative transcript levels of $r h t A$ and $r h a B$ were calculated using the preculture condition of each strain ( $\mathrm{D}$-sorbitol $100 \mathrm{mM}$; $\mathrm{t}=18 \mathrm{~h}$ ), sampled prior to the mycelium transfer to inducing and inducing/repressing conditions, as reference (*). Results are given as relative transcript ratios in logarithmic scale $(\lg (10))$. The values provided in the figures correspond to two biological replicates per culture condition. Error bars are means of three technical replicates.

transporter XltB (Sloothaak et al., 2016a). Additionally, to further investigate the RhtA transporter selectivity, D-[1- $\left.{ }^{14} \mathrm{C}\right]$-xylose and D-[ $\left.{ }^{14} \mathrm{C}(\mathrm{U})\right]$-fructose were also tested as possible substrates. As shown in Fig 7.8, the RhtA strain showed a L-rhamnose uptake rate of $5.28 \times 10^{-3} \pm 0.87 \times 10^{-3} \mathrm{nmol} \mathrm{min}^{-1} \mathrm{mg} \mathrm{DW}^{-1}$, while the transport of the methyl-pentose by the control strain could not be detected. This 
result, together with the previous findings reported in this study, confirmed that RhtA is a functional L-rhamnose transporter in A. niger. As expected, the control strain EBY.VW4000_XltB strain was able to transport D-xylose, with an uptake rate of $1.12 \times 10^{-3} \pm 0.31 \times 10^{-3} \mathrm{nmol} \mathrm{min}^{-1} \mathrm{mg} \mathrm{DW}^{-1}$, while the RhtA expressing strain could not. Finally, radiolabeled D-fructose uptake measurements confirmed the observations previously done in the functional complementation studies performed for XltB and RhtA: the EBY.VW4000_RhtA transformant could transport D-fructose with an uptake rate of $0.37 \times 10^{-3} \pm 0.06 \times 10^{-3} \mathrm{nmol} \mathrm{min}^{-1} \mathrm{mg} \mathrm{DW}$, while D-fructose uptake by the XltB strain could not be detected. The L-rhamnose uptake rate determined for RhtA is significantly lower than that one reported for the $E$. coli L-rhamnose transporter (Tate et al., 1992), however, the experimental approach used by Tate et al., which performed overnight cultivations of different $E$. coli strains (WT and L-rhamnose negative strains) for that purpose, make a comparison difficult. The L-rhamnose uptake rate determined for RhtA was, however, comparable to the maximum sugar uptake rate determined for other fungal MFS transporters, like the D-xylose transporters GXS1 from Candida intermedia (Young et al., 2012) or XylH from Debaryomyces hansenii (Ferreira et al., 2013).

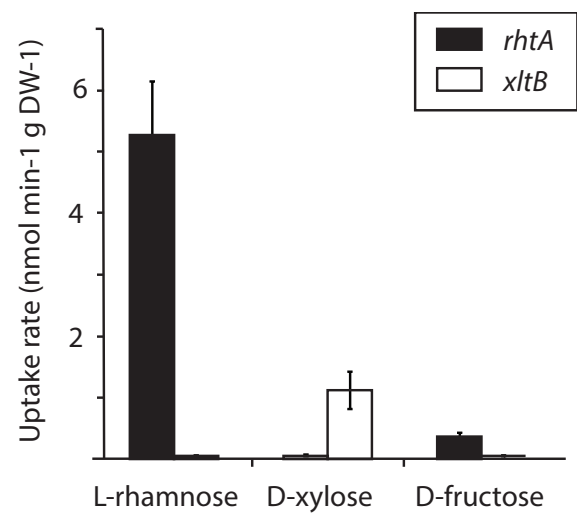

FIGURE 7.8. Functional characterization of $\boldsymbol{A}$. niger RhtA sugar transporter. Uptake at high cell density of radiolabeled substrates by $S$. cerevisiae EBY.VW4000 expressing the Aspergillus niger Lrhamnose transporter gene $r h t A$ (black bars) or D-xylose transporter gene $x l t B$ (open bars). Radiolabeled L-rhamnose, D-xylose or Dfructose were added at a final concentration of $20 \mu \mathrm{M}$. 


\subsubsection{Deletion analysis of the A. niger $r h t A$ and $r h a B$ genes}

To further understand the biological role of the L-rhamnose transporter and the hypothetical $\alpha$-L-rhamnosidase in A. niger, deletion strains of rhtA (JS16) and $r h a B$ (JS19) were constructed (see Materials and Methods section for construction details). In each case, two knockout strains were isolated, and their growth phenotype was studied by plating them on minimal media supplemented either with D-glucose ( $1 \% \mathrm{w} / \mathrm{v})$, L-rhamnose ( $1 \% \mathrm{w} / \mathrm{v})$ or rhamnogalacturonan I (RG1) $(1 \% \mathrm{w} / \mathrm{v})$ as sole carbon source. The N402 wild type strain and the regulator mutant $\Delta r h a R$ were used as controls. Growth in D-glucose was comparable, whereas clear differences could be observed in L-rhamnose plates (Fig 7.9). In the presence of of the methyl-pentose, the $\Delta r h a R$ mutants did not grow (as was described by Gruben et al. (2014)). The two $\Delta r h t A$ mutants were severely affected, showing less growth and sporulation, while the $\Delta r h a B$ transformants showed a normal growth. The growth reduction observed with the $\Delta r h t A$ mutants suggests a relevant role for RhtA in L-rhamnose uptake. The fact that the $\Delta r h t A$ mutants are still able to grow in the presence of L-rhamnose as sole carbon source indicates that $A$. niger must possess at least one other transporter capable of transporting the monosaccharide. All strains grew poorly in the presence of RG1, although slightly less growth could be observed in the $\Delta r h a R$ mutant.

\subsection{Discussion}

The prediction of substrate specificity of (sugar) transporters by using general classification systems is difficult, and traditional methods based on shared primary sequence similarity (e.g. the standard Blast algorithm) are in many cases not precise enough (Mishra et al., 2014; Sloothaak et al., 2016a). The use of profile hidden Markov models (HMM) to segregate sugar transporter proteins based on their substrate has been shown as an effective approach, however their precision largely depends on the availability of a consistent training set of biochemically characterized proteins with the function of interest (Sloothaak et al., 2015, 2016a). In this study we aimed, for the first time, to identify a eukaryotic L-rhamnose transporter and consequently, no eukaryotic examples of L-rhamnose transporters were available in the literature. Bacterial examples of L-rhamnose transporters from E. coli and Salmonella typhimurium identified in the early 1990s (Tate et al., 1992) belong to the TC 2.A.7.6 transporters family, which has no similarity with MFS transporters, and have not been associated to the transport of sugars in eukaryotic organisms. To identify putative transporters specific for L-rhamnose we have taken advantage of the fact that $A$. 


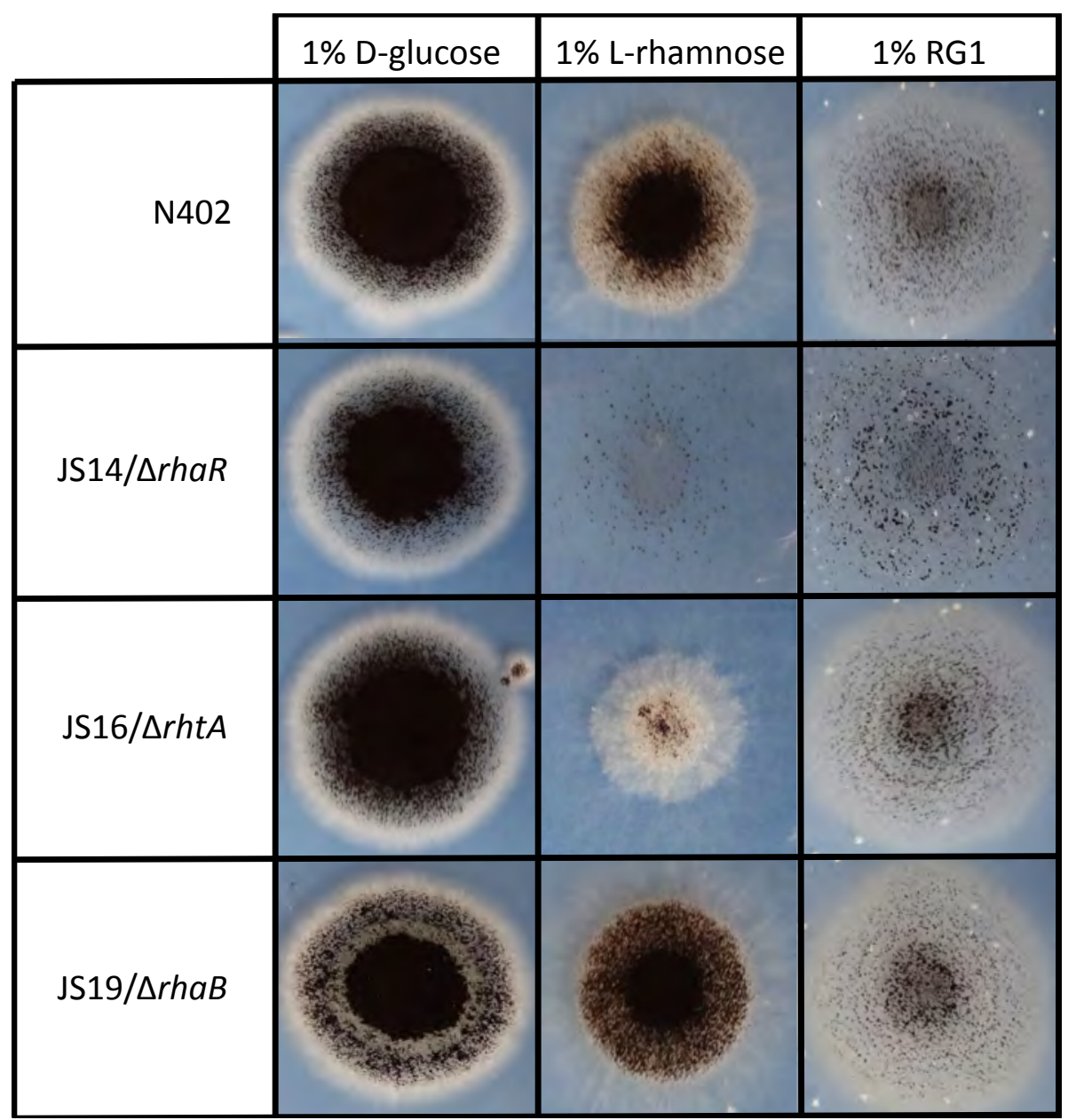

Figure 7.9. Phenotype analysis of A. niger strains N402 (WT), JS14 ( $\triangle$ rhaR), JS16 ( $\triangle$ rhtA) and JS19 ( $\triangle$ rhaB $)$. A. niger strains were plated on minimal media supplemented either with D-glucose $(1 \% ; \mathrm{w} / \mathrm{v})$, L-rhamnose (1\%; w/v) or rhamnogalacturonan I (RG1) (1 $\%$; w/v) as sole carbon source, and cultured for 144 hours. Mutants with the same gene deleted showed the same growth pattern; the figure depicts only one representative knockout strain per gene.

niger possesses complex regulatory circuits that control tightly the expression of protein sets, including extracellular enzymes, transporter proteins and metabolic enzymes, specific for the utilization of different sugars (de Souza et al., 2013). Therefore, the analyses of global transcriptomic and proteomic responses of the fungus to a variety of specific culture conditions can be useful approaches to get 
insights in the specific structural elements, including specific sugar transporters, required for the utilization of specific carbon sources. The activation of structural genes involved in L-rhamnose utilization by A. niger and other fungi have been shown to require the presence of an inducing carbon source which can be either L-rhamnose or pectic polysaccharides (Gruben et al., 2014; Koike et al., 2013; Pardo and Orejas, 2014). Assuming that the above also holds for L-rhamnose specific transporters, RhtA was selected by applying stringent differential protein expression criteria. Heterologous expression of candidate transporters in the $S$. cerevisae monosaccharide transporter null strain EBY.VW4000 has been a very effective tool to study the function of single monosaccharide transporters in isolation. Although this strain naturally only utilizes hexoses like D-glucose and D-fructose, it has been amended to metabolize D-xylose or L-arabinose (Becker and Boles, 2003; Kötter and Ciriacy, 1993; Tantirungkij et al., 1993). Accordingly, the strain can be used to screen for L-arabinose and D-xylose transporters. In a similar manner, a microbial L-rhamnose utilisation pathway transferred to the EBY.VW4000 strain would allow screening for L-rhamnose transporters. By using an alternative approach, we managed to get important insights in the possible role of $A$. niger RhtA as a functional L-rhamnose transporter. First, we showed that RhtA is expressed as a functional transporter able to transport D-fructose. Subsequently, we determined that L-rhamnose had an extraordinary ability to specifically inhibit growth of the EBY.VW4000_RhtA strain, even at micromolar levels. This fact, plus additional insights observed at genomic, transcriptomic and proteomic level gave strong indications that RhtA acts as a specific transporter for L-rhamnose, but did not provide direct evidence. To assay transport capacity and quantify uptake kinetics, radiolabeled sugar uptake experiments can be performed with the mentioned yeast strain (Colabardini et al., 2014; dos Reis et al., 2013; Leandro et al., 2013; Sloothaak et al., 2015; Subtil and Boles, 2011; Wahl et al., 2010). This approach was followed in the present study as a final step to prove the ability of RhtA to transport L-rhamnose.

Besides the ability of RhtA to transport L-rhamnose, we have obtained important insights into the biological role of the transporter. The tandem localization of $r h t A$ with the $\alpha$-L-rhamnosidase coding gene $r h a B$, and their coordinated transcriptional response to the presence of L-rhamnose, indicated that both proteins could have a joint action in releasing and transporting the sugar. We also investigated if, besides $r h a B$, additional hypothetical $\alpha$-L-rhamnosidase genes (1180185/An08g09140, 1160525/An18g04800, 1132057/An01g06620, 1180185/ An15g04530, 1126821/An04g09070, 1134376/An07g00240, and 1165677/An10g00290; Protein accession numbers in ATCC 1015 and CBS 513.88 genomes) were co- 
localized with sugar transporters and noticed that the co-expressed rhaB-rhtA tandem was a unique case in $A$. niger.

The transcriptional profile of $r h t A$ and $r h a B$ genes also suggests a coordinated role for the utilization of the deoxy sugar. In the presence of an initial L-rhamnose concentration of $1 \mathrm{mM}$ their expression was strongly induced while the sugar was being consumed. Concentrations even lower than $1 \mathrm{mM}$ might therefore also induce this system, as it occurs in the case of the D-xylose utilisation system in A. niger, where a concentration of $0.1 \mathrm{mM} D$-xylose already exerts a strong activation of structural genes like $x \ln B$ and $x \ln D$ (van der Veen et al., 2009).

Regarding the expression regulation of the transporter and the $\alpha$-L-rhamnosidase genes mediated by RhaR, we also analyzed the microarray expression data of $A$. niger WT and a $\Delta r h a R$ strain grown on L-rhamnose (accession number GSE51023) recently deposited by Gruben et al. at GEO (Gruben et al., 2014), observing that in this independent study using a different setup $r h t A$ (An12g05710) and $r h a B$ (An12g05700) were not induced in a RhaR knockout strain. Therefore, both microarray (Gruben et al.) and RT-qPCR (this study) data analysis of two independent studies indicated that RhaR is responsible for $r h t A$ and $r h a B$ transcriptional activation. However, although the effect produced by RhaR in the regulation of these genes is very clear, as can be observed in Fig 7.7 their induction was not fully abolished in the $\Delta r h a R$ strain. This might indicate the influence of another transcriptional regulator that responds to the presence of L-rhamnose. Previously, a second transcriptional factor (FST14) was suggested to be involved in the regulation of the $P$. stipitis pectinolytic network (Koivistoinen et al., 2012), and this could also be the case in A. niger, as recently suggested by Gruben et al. (Gruben et al., 2014).

In the presence of L-rhamnose plus D-glucose, $r h t A$ and $r h a B$ were heavily repressed in the wild type strain, and only partly derepressed in the $\Delta c r e A$ mutant. While this result suggests a role of CreA in controlling the expression of $r h t A$ and $r h a B$, as reported before for other sugar utilisation systems (Ademark et al., 2001; de Vries et al., 1999; Niu et al., 2015; Ruijter et al., 1997a), a different glucose-repression mechanism seems to have a major role in the transcriptional regulation of these genes. The existence of CreA-independent glucose repression mechanisms controlling the regulation of $\alpha$-L-rhamnosidase genes (TamayoRamos et al., 2012), and the phenylacetic acid uptake system (Cubero et al., 2000), has been previously reported in A. nidulans.

The isolation of $r h t A$ and $r h a B$ deletion strains allowed us to investigate the relevance of both proteins in A. niger. On one hand, the absence of RhtA produced a growth and sporulation defect in the $\Delta r h t A$ mutants on L-rhamnose containing 
media, underpinning the relevant biological role of RhtA for transport of Lrhamnose. Similar phenotype analyses performed in filamentous fungi, where different sugar transporter mutant strains were studied, produced disparate results. In some cases, an altered growth phenotype could not be detected in plate assays containing the transporter's specific substrate (Forment et al., 2006, 2014; Vankuyk PA et al., 2004; Wahl et al., 2010), which is probably due to overlapping substrate specificities. In this regard, the most notorious case corresponds to $S$. cerevisiae, where many genes had to be disrupted before its ability to transport D-glucose was abolished (Wieczorke et al., 1999). However, as we observed in the present study, the absence of certain transporters in filamentous fungi has also been shown to be accompanied with clear growth defects (Galazka et al., 2010; Huang et al., 2015; Zhang et al., 2013). On the other hand, the absence of $r h a B$ did not affect negatively the mutant growth in the presence of RG1. This result is not surprising, bearing in mind that $\alpha$-L-rhamnosidase genes appear to be quite redundant in the $A$. niger genome.

RhtA is, according to our knowledge, the first functionally validated eukaryotic transporter containing a FucP domain structure. It is also the first eukaryotic L-rhamnose transporter functionally validated to date, therefore this study provides major insights about the utilisation of this monosaccharide by fungi. The identification of RhtA will also have an impact in the design of new microbial strains using L-rhamnose-rich biomass as feedstocks, like pectic polysaccharides from plants (Edwards and Doran-Peterson, 2012), or ulvan from green seaweeds (Behera et al., 2014; Lahaye and Robic, 2007), for the production of fuels and chemicals.

\subsection{Materials and Methods}

\subsubsection{Strains and growth conditions}

The Aspergillus niger strains used in this study were N400 (NRRL3, ATCC9029, CBS120.49), N402 (cspA1) (Bos et al., 1988), NW283 (fwnA1; cspA1; lysA7; pyrA6; creAd4) (Ruijter et al., 1997a), MA169.4 (cspA1, kusA::DR-amdS-DR, pyrG ( $^{-}$) (Carvalho et al., 2010), and its derivatives JS14, ( $\Delta r h a R)$, JS16 ( $\Delta r h t A)$ and JS19 $(\triangle r h a B)$ constructed in this study. A. niger spores were generated on complete medium (CM) plates. Mycelial biomass for transfer experiments was produced in 18 hour pre-cultures after the inoculation of $10^{6}$ spores $\mathrm{mL}^{-1}$ in culture medium containing: $6 \mathrm{~g} \mathrm{~L}^{-1}$ (w/v) $\mathrm{NaNO}_{3}, 1.5 \mathrm{~g} \mathrm{~L}^{-1}$ (w/v) $\mathrm{KH}_{2} \mathrm{PO}_{4}, 0.5 \mathrm{~g} \mathrm{~L}^{-1}$ (w/v) $\mathrm{KCl}$, $0.5 \mathrm{~g} \mathrm{~L}^{-1}(\mathrm{w} / \mathrm{v}) \mathrm{MgSO}_{4} \mathrm{H}_{2} \mathrm{O}$ and Vishniac salts (Pontecorvo et al., 1953; Vishniac 
and Santer, 1957), with $5 \mathrm{~g} \mathrm{~L}^{-1}$ (w/v) yeast extract, D-sorbitol $100 \mathrm{mM}$, and the appropriate supplements to complement auxotrophic mutations (initial pH 6.0).

For the plasma membrane proteomics analysis, equal amounts of water-rinsed mycelium of the wild type strain A. niger N400 were transferred to 1-liter benchtop fermenters (Sartorius) with $750 \mathrm{~mL}$ of minimal medium (MM) containing: $6 \mathrm{~g} \mathrm{~L}^{-1}$ (w/v) $\mathrm{NaNO}_{3}, 1.5 \mathrm{~g} \mathrm{~L}^{-1}$ (w/v) $\mathrm{KH}_{2} \mathrm{PO}_{4}, 0.5 \mathrm{~g} \mathrm{~L}^{-1}$ (w/v) $\mathrm{KCl}, 0.5 \mathrm{~g} \mathrm{~L}^{-1}$ (w/v) $\mathrm{MgSO}_{4} \mathrm{H}_{2} \mathrm{O}$, Vishniac salts, and one of the following carbon sources: Larabinose $5 \mathrm{mM}$, D-mannose $5 \mathrm{mM}$, D-sorbitol $100 \mathrm{mM}$ plus D-xylose $0.1 \mathrm{mM}$, and L-rhamnose $5 \mathrm{mM}$. Two biological replicates per condition were studied. Fermenters were kept at $30^{\circ} \mathrm{C}$, stirred at $1^{\prime} 000 \mathrm{rpm}$ and aerated with filtered air $\left(0.6 \mathrm{~L} \mathrm{~min}^{-1}\right)$, keeping oxygen levels over $60 \%$. The initial $\mathrm{pH}$, set at 4.0, was allowed to drop until $\mathrm{pH} 3.5$ and kept constant afterwards by sodium hydroxide addition. These culture conditions were also used in the study of $r h t A$ transcriptional response to D-sorbitol $100 \mathrm{mM}$, L-rhamnose $5 \mathrm{mM}$, and D-fructose $5 \mathrm{mM}$; and also in the time course fermentations in cultures containing D-sorbitol 100 $\mathrm{mM}$, L-rhamnose $1 \mathrm{mM}$, or L-rhamnose $5 \mathrm{mM}$.

For the analysis of the regulatory mechanisms controlling $r h t A$ and $r h a B$, the $A$. niger mycelial biomass of the strains N402, NW283 ( $\triangle c r e A)$ and JS14 ( $\triangle r h a R$ ) was produced in 18 hour pre-cultures (2 Erlenmeyer flasks per strain) after the inoculation of $10^{6}$ spores $\mathrm{mL}^{-1}$ in culture medium containing: $6 \mathrm{~g}$ $\mathrm{L}^{-1}$ (w/v) $\mathrm{NaNO}_{3}, 1.5 \mathrm{~g} \mathrm{~L}^{-1}$ (w/v) $\mathrm{KH}_{2} \mathrm{PO}_{4}, 0.5 \mathrm{~g} \mathrm{~L}^{-1}$ (w/v) $\mathrm{KCl}, 0.5 \mathrm{~g} \mathrm{~L}^{-1}$ (w/v) $\mathrm{MgSO}_{4} \mathrm{H}_{2} \mathrm{O}$, Vishniac salts, $1 \mathrm{~g} \mathrm{~L}^{-1}$ (w/v) yeast extract, $100 \mathrm{mM}$ D-sorbitol, and the appropriate supplements to complement auxotrophic mutations (initial $\mathrm{pH}$ 6.0). Equal amounts of mycelium from each strain were then transferred to $100 \mathrm{~mL}$ Erlenmeyer flasks with the same medium composition, containing 5 mM L-rhamnose or $5 \mathrm{mM}$ L-rhamnose plus $50 \mathrm{mM}$ D-glucose as carbon sources. Cultures were grown in an orbital shaker for two hours at $30{ }^{\circ} \mathrm{C}$ and $225 \mathrm{rpm}$.

The phenotype analysis of the different $A$. niger mutant strains obtained in this study was done in agar plates, containing MM plus the appropriate supplements and carbon sources.

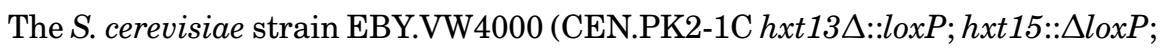

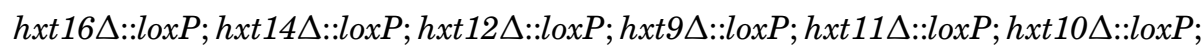

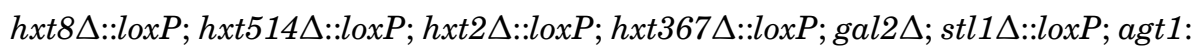
:loxP; ydl247w $\Delta:: l o x P ; y j r 160 c \Delta:: l o x P$ ) (Wieczorke et al., 1999), that was used in this study for the functional validation of the $r h t A$ gene, was grown at 30 ${ }^{\circ} \mathrm{C}$ and maintained in solid complete medium containing $10 \mathrm{~g} \mathrm{~L}^{-1}(\mathrm{w} / \mathrm{v})$ of yeast extract, $20 \mathrm{~g} \mathrm{~L}^{-1}$ (w/v) of peptone and $20 \mathrm{~g} \mathrm{~L}^{-1}$ (w/v) of maltose. The EBY.VW4000 derived strains obtained in the present study where grown in liquid minimal 
medium (MM) containing $6.7 \mathrm{~g} \mathrm{~L}^{-1}(\mathrm{w} / \mathrm{v})$ of yeast nitrogen base with ammonium sulphate (w/o amino acids) (Difco), supplemented with leucine ( $\left.30 \mathrm{mg} \mathrm{L}^{-1} ; \mathrm{w} / \mathrm{v}\right)$, tryptophan (20 mg L $\left.{ }^{-1} ; \mathrm{w} / \mathrm{v}\right)$ and histidine $\left(20 \mathrm{mg} \mathrm{L}^{-1} ; \mathrm{w} / \mathrm{v}\right)$, and using $20 \mathrm{~g} \mathrm{~L}^{-1}$ (w/v) of maltose as carbon source. The $S$. cerevisiae wild type strain CEN.PK2-1C

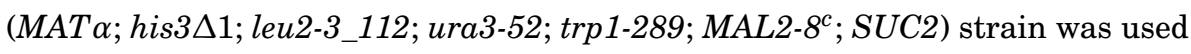
as a control strain.

For the yeast spot assays, the different $S$. cerevisiae strains were grown overnight as mentioned above and harvested in exponential phase by centrifugation. Cells were then diluted to the following optical densities at $600 \mathrm{~nm}\left(\mathrm{OD}_{600}\right)$ : $1,0.1,0.01$ and 0.001 , and subsequently $5 \mu \mathrm{L}$ droplets were spotted on $1.5 \%$ agar plates containing MM plus the appropriate supplements and different carbon sources.

For radiolabelled sugars uptake experiments $S$. cerevisiae strains were cultured in baffled flasks with MM supplemented with methionine and arginine for enhanced growth, and histidine, leucine and tryptophan to complement auxotrophic mutations. Incubations were done in an orbital shaker at $30{ }^{\circ} \mathrm{C}$ and 225 rpm.

\subsubsection{A. niger membrane associated proteome purification, quality control analysis, sample preparation for LC-MS/MS and proteomics data analysis}

The $A$. niger membrane proteome preparation and purification was performed as described (Sloothaak et al., 2015). A. niger mycelium samples (2-3 g, pressdried), washed and resuspended in $20 \mathrm{mM}$ HEPES buffer ( $\mathrm{pH} 7.6$ ) containing $150 \mathrm{mM} \mathrm{NaCl}$ and protease inhibitor cocktail for yeast \& fungi (Sigma-Aldrich), were mechanically disrupted using a French press (8'000 psi). Three differential centrifugation steps, at low (500 g), medium (5’000 g) and high speed ( $~ 85^{\prime} 000$ $\mathrm{g}$ ), were performed to pellet light organelles (P3). P3 pellets were resuspended using a Dounce homogenizer in $1 \mathrm{~mL}$ of $20 \mathrm{mM}$ HEPES buffer ( $\mathrm{pH} 7.6$ ), containing $250 \mathrm{mM}$ sucrose. The P3 suspensions were subsequently overlaid in a discontinuous sucrose density gradient, prepared by layering successive solutions, with decreasing sucrose concentrations ( $6 \times 1 \mathrm{~mL} ; 1.20 \mathrm{M}$ to $0.70 \mathrm{M})$, upon one another. Sucrose density gradients were centrifuged ( $100,000 \mathrm{~g}-60 \mathrm{~min})$ to isolate different membrane-associated fractions from $\mathrm{P} 3$ pellet. Five fractions were obtained (P3A, P3B, P3C, P3D and P3E). The plasma membrane (PM) marker vanadate-sensitive $\mathrm{H}^{+}$ATPase and the mitochondrial membrane cytochrome $\mathrm{c}$ oxidase activities were then measured in the initial cell free extract, the P3 pellet 
and the P3A to P3E fractions derived from it. Compared to the cell free extract, the P3 pellet was 2.4 to 3.2 times enriched in plasma membranes. No further enhanced PM enrichment was found in the P3A to P3E fractions, therefore the P3 pellets were considered to be more optimal for the analysis of plasmalemma proteins, that were further processed and subjected to shotgun proteomics analysis. Cytochrome c oxidase activity measurements were performed using the "Cytochrome c Oxidase Assay Kit" from Sigma-Aldrich (CYTOCOX1), following the user manual. The vanadate-sensitive $\mathrm{H}^{+}$ATPase enzyme assay was performed as described previously (Janicka-Russak et al., 2012).

The protocol used in order to prepare membrane proteins for LC-MS/MS analysis has been described in detail previously (Sloothaak et al., 2015). LC-MS/MS analyses were done at Radboud Proteomics Centre as described previously (Rajala et al., 2015). The obtained raw mass spectrometry proteomics data was deposited to the ProteomeXchange Consortium via the PRIDE partner repository with the dataset identifier PXD004909. The analysis of the LC-MS/MS spectra obtained from the proteomics experiment, were identified and quantified using the MaxQuant software (Cox and Mann, 2008), as described (Sloothaak et al., 2015).

\subsubsection{Construction of S. cerevisiae EBY.VW4000 strain expressing A. niger rhtA gene}

The chemically synthesized $r h t A$ cDNA coding sequence (A. niger ATCC 1015 JGI prot. ID: 1119135) was PCR amplified from plasmid using primers HE_rhtA_FW and HE_rhtA_RV (S2 Table), using Phusion polymerase (ThermoFisher Scientific) following the manufacturers protocol. The fragment was then digested with SpeI and XhoI and cloned into the $S$. cerevisiae expression vector p426HXT7-6His, linearized with the same restriction enzymes, under the control of the constitutive promoter HXT7p and the terminator CYC1t. Transformation of S. cerevisiae EBY.WV4000 was performed as described previously (Gietz and Woods, 2002).

\subsubsection{Construction of JS14 ( $\triangle r h a R)$, JS16 $(\Delta r h t A)$ and JS19 $(\triangle \boldsymbol{r h a B})$}

Using the split-marker approach, the previously identified L-rhamnose regulator gene rhaR (JGI ATCC 1015 Prot ID 1116273) (Gruben et al., 2014), the rhamnose transporter gene $r h t A$ (JGI ATCC 1015 Prot ID 1119135), or the putative rhamnosidase gene $r h a B$ (JGI ATCC 1015 Prot ID 131668) were deleted from the genome of the MA169.4 strain (isogenic of N402), which is defective in the 
Non-Homologous End-Joining (NHEJ) pathway through a transiently silenced kusA gene (Carvalho et al., 2010; Meyer et al., 2007). A schematic representation of the four experimental steps required can be found in S3 Fig, PCR results that confirm the correct deletion of the genes from the genome can be found in $\mathrm{S} 4 \mathrm{Fig}$ and primers used are listed in S2 Table.

As an example we describe the construction of JS14 ( $\triangle r h a R)$ knockout strains, the other strains were constructed in the same manner with the corresponding primers. First, homologous regions were amplified from A. niger N402 genomic DNA using primers KO_rhaR5'FW with KO_rhaR5'_RV and KO_rhaR3'_FW with KO_rhaR3'_RV, and the marker gene was amplified from pAO4-13 using primers KO_pyrG_FW and KO_pyrG_RV. Second, these three fragments were used as template to create marker-flank fusion fragments using primers KO_rhaR5'_FW with KO_pyrG2_RV and KO_pyrG2_FW with KO_rhaR3'_RV. Third, the resulting fragments were used to transform MA169.4 as previously described (Kusters-van Someren et al., 1991). Single A. niger transformant colonies were purified and the transiently silenced $k u s A$ gene was restored on MM plates containing fluoroacetic acid (FAA). Finally, correct marker localization in the strain JS14 ( $\triangle r h a R$ ) was checked by PCR using genomic DNA as template, and the primer pairs CH_locusrhaR_FW with KO_pyrG2_RV and KO_pyrG2_FW with $\mathrm{CH}$ _locusrhaR_RV. Deletion of the $r h a R$ gene was confirmed by PCR using the primers CH_rhaR_FW and $\mathrm{CH} \_r h a R \_R V$.

\subsubsection{RNA extraction and transcriptional analysis of $r h t A$ and rhaB genes}

Mycelium samples were disrupted with glass beads in a Fastprep-24 instrument, and RNA was isolated using a Maxwell 16 instrument using the Maxwell 16 LEV simplyRNA kit (Promega). Reverse transcription and qPCR analysis were performed following the protocols and instruments described in Mach-Aigner et al. (2012). In short, after treatment with DNase I, cDNA was synthesized from $0.45 \mu \mathrm{g}$ RNA using the RevertAid H Minus First Strand cDNA synthesis kit (Thermo Fisher). All reactions were performed according to the manufacturer's instructions. All quantitative PCRs (qPCRs) were performed in triplicate in a Rotor-Gene 3000 cycler (Qiagen). The amplification mixture (final volume, 15 $\mu \mathrm{L}$ ) contained 7.5 $\mu \mathrm{L}$ of $2 \mathrm{x}$ ABsolute QPCR SYBR Green mix, $100 \mathrm{nM}$ forward and reverse primers and $2.5 \mu \mathrm{L}$ cDNA (diluted 1:100). The primers used for qPCR analysis were designed using the software QuantPrime (Arvidsson et al., 2008), and are listed in S2 Table. Each run included a no-template control and 
a no-amplification control ( $0.015 \%$ SDS added to the reaction mixture). The cycling conditions comprised a 15 min initial polymerase activation at $95{ }^{\circ} \mathrm{C}$, followed by 40 cycles of $95{ }^{\circ} \mathrm{C}$ for $15 \mathrm{~s}, 59{ }^{\circ} \mathrm{C}$ for $15 \mathrm{~s}$, and $72{ }^{\circ} \mathrm{C}$ for $15 \mathrm{~s}$. The previously described histone-like gene hist transcript (A. niger ATCC 1015 gene ID 207921) and the Golgi transporter gene (A. niger CBS 513.88 An02g04120) were used as reference for normalization of the expression data (Mach-Aigner et al., 2012; van der Veen et al., 2009). Dissociation (or melting) curve analysis was performed on each $\mathrm{qPCR}$ reaction to confirm that the primer pairs used produced a single amplification product. Results are given as relative transcript ratios in logarithmic scale $(\lg (10))$. The values provided in the figures correspond to two biological replicates per strain and culture condition.

\subsubsection{Sugars analysis}

Sugars present in the A. niger culture supernatants were measured through highpressure liquid chromatography (HPLC) analysis. A Thermo Accela equipped with a Shodex KC-811 column, coupled to a refractive index detector (Spectrasystem RI-150, sample frequency $5.00032 \mathrm{~Hz}$ ) and a UV-VIS detector (Spectrasystem UV1000, $\lambda=210 \mathrm{~nm}$ ), was used. Separations were performed by isocratic elution with $0.01 \mathrm{~N} \mathrm{H}_{2} \mathrm{SO}_{4}$, at a flow rate of $0.8 \mathrm{~mL} \mathrm{~min}^{-1}$. Crotonate $(6 \mathrm{mM})$ was used as an internal standard.

\subsubsection{Radiolabeled sugar uptake determinations}

Sugar uptake assays were performed as described previously, with minor adjustments (Walsh et al., 1994). Liquid cultures using MM, supplemented with methionine (20 mg L $\left.{ }^{-1} ; \mathrm{w} / \mathrm{v}\right)$, arginine (20 $\left.\mathrm{mg} \mathrm{L}^{-1} ; \mathrm{w} / \mathrm{v}\right)$, leucine $\left(30 \mathrm{mg} \mathrm{L}^{-1} ; \mathrm{w} / \mathrm{v}\right)$, tryptophan $\left(20 \mathrm{mg} \mathrm{L}^{-1} ; \mathrm{w} / \mathrm{v}\right)$ and histidine $\left(20 \mathrm{mg} \mathrm{L}^{-1} ; \mathrm{w} / \mathrm{v}\right)$, with $1 \%(\mathrm{w} / \mathrm{v})$ maltose as carbon source, were inoculated with strains EBY.VW4000_XltB (control), and EBY.VW4000_RhtA, and incubated for 5 days. Cells were then harvested by centrifugation (4000 g, $10 \mathrm{~min}$ ), washed with $50 \mathrm{~mL}$ ice-cold ultrapure water, and washed again with ice-cold PBS ( $\mathrm{pH}$ 6.5). Cells were then resuspended in PBS ( $\mathrm{pH}$ 6.5), divided in $40 \mu \mathrm{L}$ aliquots, and kept on ice.

Aliquots were incubated for $5 \mathrm{~min}$ at $30{ }^{\circ} \mathrm{C}$ before the uptake assay was started. To start the assay, $10 \mu \mathrm{L}$ of a $100 \mathrm{mM} \mathrm{L-}\left[{ }^{3} \mathrm{H}(\mathrm{G})\right]-$ rhamnose, D-[ $\left.{ }^{14} \mathrm{C}(\mathrm{U})\right]-$ fructose or D-[1- $\left.{ }^{14} \mathrm{C}\right]$-xylose solution (Campro Scientific, Veenendaal) was added. After exactly 20 seconds the reaction was quenched by the addition of $1 \mathrm{~mL}$ of ice-cold wash buffer (PBS, pH 6.5, with $500 \mathrm{mM}$ of the corresponding non-labeled substrate solution), followed by a vacuum filtration step $(0.45 \mu \mathrm{m}$ HV filters, 1225 
sampling manifold, Millipore), and two subsequent washing steps with $5 \mathrm{~mL}$ of ice-cold washing buffer. After drying for $5 \mathrm{~min}$ in the vacuum manifold, the filters were transferred to scintillation vials with $5 \mathrm{~mL}$ scintillation liquid (Ultima Gold, Perkin Elmer), and activity was counted (Packard Tricarb 1600TR). All reactions were performed in triplicate. Negative control reactions, where quenching was done before substrate addition and without incubation, were performed for each reaction.

\subsubsection{Bioinformatics analysis}

DNA and protein sequences were obtained from the JGI A. niger ATCC 1015 genome database (Nordberg et al., 2013). Retrieved sequences were subsequently used in additional searches using the BLAST tools at the NCBI database. Protein transmembrane-aware multiple alignments were done using a PRALINE, incorporating TMHMM2.0 transmembrane helix prediction tool (Krogh et al., 2001; Simossis and Heringa, 2005). SignalP4.1 was used to detect the signal peptide for secretion in the RhaB protein (Petersen et al., 2011).

\subsection{Acknowledgements}

We would like to thank Dr Eckhard Boles for providing us the $S$. cerevisiae strain EBY.VW4000, and Mark Arentshorst for providing us the A. niger MA169.4 strain. We also thank Tom Schonewille, Maarten Bezembinder, Frits van Charante and Mike Schilders for their contribution to this work.

\section{Supplementary information}

Supplementary files for this chapter can be found online at:

http://journals.plos.org/plosgenetics/article?id=10.1371/journal.pgen.1006468 



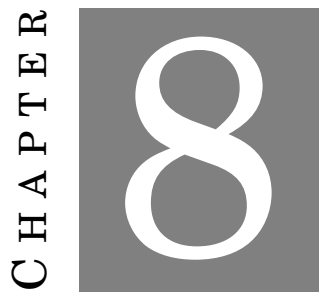

General Discussion 


\subsection{Thesis breakdown}

The purpose of this thesis was to increase the understanding of Aspergillus niger as biotechnological work horse, thereby providing the tools and knowledge needed to turn it into a more effective cell-factory for the production of citrate and other organic acids. As described in Chapter 1, there are 3 main elements that need to be considered regarding improvement of microbial cell-factories: substrate import, conversion of the substrates to the product of interest, and export of the product of interest (Figure 8.1).

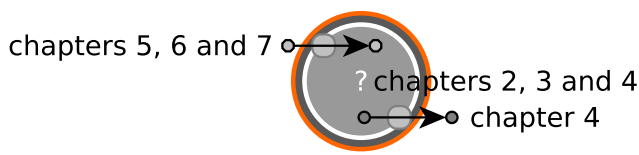

FIGURE 8.1. Thesis breakdown. Each chapter of this thesis contributes to either the identification or the understanding of one or more of 3 key elements in microbial cell-factories; substrate import (Chapters 5, 6 and 7), substrate conversion to product of interest (Chapters 2, 3, and 4) and product export (Chapter 4).

As can be seen by the number of chapters dedicated to substrate import (Chapters 5, 6 and 7), identification of $A$. niger sugar importers was successful. The use of combined in silico and in vivo approaches yielded various validated glucose and xylose transporters (Chapters 5 and 6), and one validated rhamnose transporter (Chapter 7). Identification of organic acid exporters proved to be a more intricate challenge. We succeeded in identifying one organic acid transporter, which was confirmed to be an A. niger citrate exporter (Chapter 4). Most importantly, multiple attempts to identify both an Rhizopus delemar fumarate and A. niger citrate exporter, and especially analyses of the failures in doing so, led to a different way of looking at the complex challenge of fungal organic acid production, providing further insights into both fumarate and citrate production in $R$. delemar and $A$. niger (Chapters 2, 3 and 4).

Note that, as introduced in Chapter 1, biotechnological citrate production using $A$. niger is still subject to fierce competition, and "industrial citric acidproducing strains of this fungus are among the most secretly kept organisms in biotechnology" (Karaffa and Kubicek, 2003). As a result, product titers reported in academic research (20 - $115 \mathrm{~g} / \mathrm{L}$ (Yin et al., 2015)) are far from maximum titers reported for industrial A. niger citrate production ( $>200 \mathrm{~g} / \mathrm{L}$ ). In this thesis, the focus lies on understanding the system, laying the foundations for future improvement of $A$. niger as biotechnological cell-factory. 


\subsection{Fumarate production in $R$. delemar}

One of the specific objectives of this thesis was to study the possible exploitation of $A$. niger as biotechnological cell-factory for the production of fumarate; a compound it does not naturally secrete in relevant amounts. In contrast, $R$. delemar secretes fumarate in high amounts (Foster and Waksman, 1939a), which is mainly attributed to the presence of cytosolic pyruvate carboxylase (PYC) and cytosolic enzymes involved in the reductive route of the TCA (rTCA) cycle (Kenealy et al., 1986; Osmani and Scrutton, 1985) in Rhizopus spp (Figure 8.2).

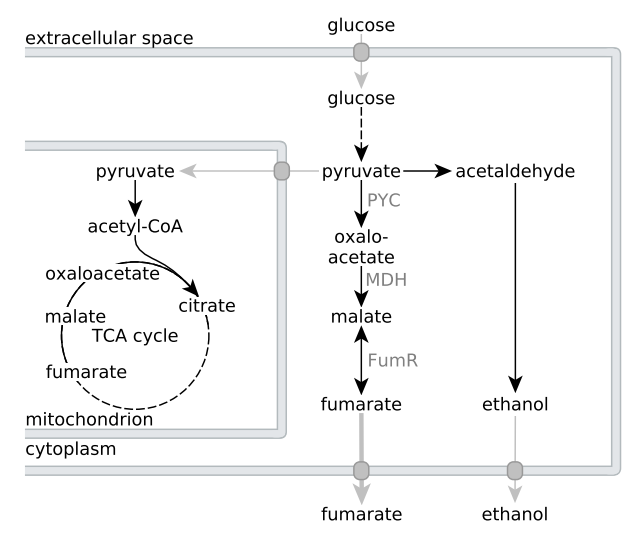

FIGURE 8.2. General consensus of metabolic pathways involved in fumarate metabolism in R. delemar. Metabolic flux of $R$. delemar is predominantly directed towards fumarate (under aerobic conditions) or ethanol (under anaerobic conditions). The enzymes of the reductive TCA cycle are indicated in the scheme: $\mathrm{PYC}=$ pyruvate carboxylase, $\mathrm{MDH}=\mathrm{L}$-malate dehydrogenase, and FumR = fumarase. Adapted from Chapter 2.

As briefly discussed in Chapter 2, a remaining controversial aspect in the scheme depicted in Figure 8.2 is the role of cytosolic fumarase (FumR). Fumarase is better known as a mitochondrial enzyme, where it catalyses the reaction from fumarate to malate as part of the TCA cycle. It has long been debated whether $R$. delemar can accumulate high concentrations of fumarate due to the presence of two differently encoded fumarases, of which the one in the mitochondria catalyses the reaction from fumarate to malate, and the one in the cytosol catalyses the reverse reaction (Goldberg et al., 2006). This theory was discarded after a study by Friedberg et al. (1995) showed the presence of only one fumarase transcript $($ fumR) in $R$. delemar. This is in line with the findings for $S$. cerevisiae, rat and human fumarases, which are all encoded by a single gene, while the enzymes are found in both the mitochondria and the cytosol depending on whether proteolytic 
cleavage of the mitochondrial targeting signal takes place or not (Sass et al., 2001; Singh and Gupta, 2006; Suzuki et al., 1989; Wu and Tzagoloff, 1987).

Another suggestion by Goldberg et al. (2006) and Song et al. (2011) was that the reaction catalysed by FumR becomes irreversible at higher fumarate concentrations, based on the finding that FumR activity was completely blocked when fumarate concentrations exceeded $2 \mathrm{mM}$ in vitro (unpublished results cited in Goldberg et al. (2006), later reconfirmed by Song et al. (2011)). However, the $\Delta_{\mathrm{r}} \mathrm{G}^{\prime}$ for the conversion of malate to fumarate at physiological conditions is $3.5 \pm 0.6 \mathrm{~kJ} \cdot \mathrm{mol}^{-1}$, suggesting higher concentrations of malate than fumarate at equilibrium (Meussen et al., 2012). Thus, accumulation of high amounts of fumarate by $R$. delemar could not unambiguously be explained by the properties of the characterised FumR. This eventually led to the suggestion of the presence of a $R$. delemar dicarboxylic acid exporter that is highly selective for fumarate, effectively "pulling" fumarate out of the cell before it can be converted to malate (Meussen et al., 2012).

\subsection{The search for the $R$. delemar fumarate exporter - transition from reductionism to holism}

\subsection{1 "Eureka!"}

As cytosolic enzymes of the rTCA cycle are also annotated in the manually curated genome-scale metabolic model of A. niger (Andersen et al., 2008), it seems reasonable to assume that heterologous expression of a fumarate specific $R$. delemar transporter would manage to pull fumarate out of $A$. niger cells in the same way as in $R$. delemar. Thus, with the task of identifying the $R$. delemar fumarate exporter, I analysed my first RNA sequencing (RNA seq) and proteomics data sets. RNA and proteins were obtained from $R$. delemar, which had been pregrown in shake flasks, and then transferred to fermentors containing no nitrogen source; an experimental setup conforming the established conditions required for extracellular fumarate accumulation (Goldberg et al., 2006; Magnuson and Lasure, 2004). Maximum contrast in fumarate yield per gram glucose consumed was achieved by modulating the amount of oxygen entering the fermentors (described in detail in Chapter 2).

The -omics data were condensed into a list of putative fumarate transporter candidates based on their transcript levels and protein abundances in the high- and low-fumarate producing conditions. A promising transporter from the RNA seq experiments translated into a protein that could be classified 
as a sodium:dicarboxylate symporter ( $R$. delemar RA 99-880 gene identifier RO3G_15608). This putative fumarate transporter was promising not only based on the expression level in the two contrasting conditions, but also due to the description for the substrates of this type of transporters (from the transporter classification database, tcdb; http://www.tcdb.org/search/result.php?tc=2.A.23):

"The members of the Dicarboxylate/Amino Acid:Cation $\left(\mathrm{Na}^{+}\right.$or $\left.\mathrm{H}^{+}\right)$Symporter (DAACS) family catalyse $\mathrm{Na}^{+}$and/or $\mathrm{H}^{+}$symport together with: (a) a Krebs cycle dicarboxylate (malate, succinate, or fumarate); (b) a dicarboxylic amino acid (glutamate or aspartate); (c) a small, semipolar, neutral amino acid (Ala, Ser, Cys, Thr); (d) both neutral and acidic amino acids or (d) most zwitterionic and dibasic amino acids."

When this transporter also appeared to be the top-candidate in the proteomics data based on its fold-change in relative protein abundance, it seemed that this was indeed the R. delemar fumarate exporter, optimistically denoted "FumT". FumT was heterologously expressed in $A$. niger to see whether we would find increased fumarate production in the transformants compared to the parent strain. The results from the initial growth experiment, performed in shake flasks, looked promising (Figure 8.3).
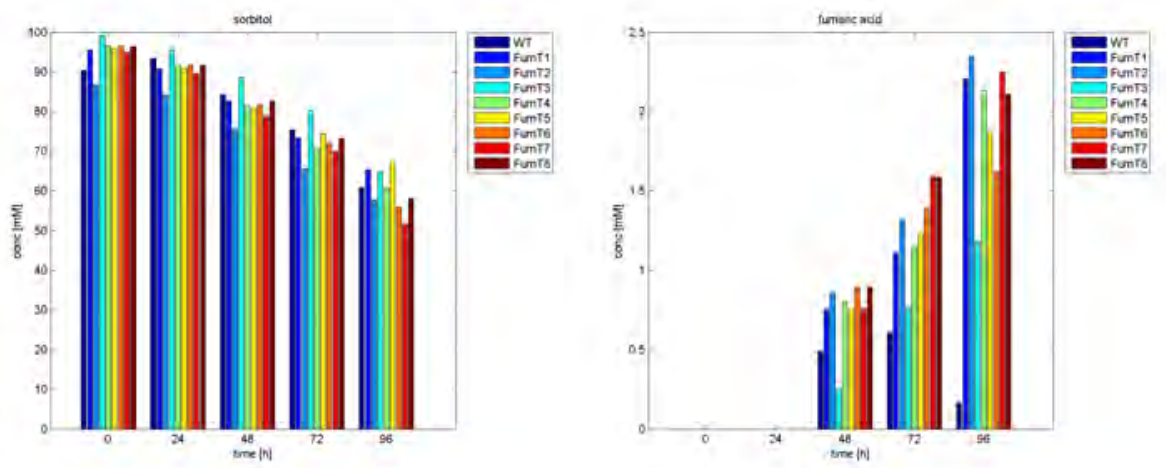

FIGURE 8.3. A. niger sorbitol consumption (left) and fumarate production (right) in initial growth experiment ${ }^{1}$. The first comparison of various $A$. niger FumT transporter mutants to the untransformed parent strain ("WT") looked promising.

\footnotetext{
${ }^{1}$ Note that these graphs were plotted at the start of my $\mathrm{PhD}$, before figuring out that one of my colleagues is colour-blind. This makes him one of the $10 \%$ of men that can not easily perceive all aspects of the data, if graphical representation of these data relies on the use of colours alone. To promote papers as a form of dissemination of our research that is accessible to a wider range of scientists, it is time to overhaul the classical choice of red-green as a representation to distinguish scientific data (see also the Editorial Seeing science in color in Nature Structural \& Molecular Biology 14, 173 (2007), doi:10.1038/nsmb0307-173).
} 


\subsection{2 "That's funny..."}

There was one (apparent) problem with the initial growth experiment (Figure 8.3). The A. niger parent strain used for transformation was the gluconate and oxalate non-producing strain used throughout this thesis, referred to as $A$. niger NW186, which is a derivative of A. niger NW185 (cspA1, fwnA1, goxC17, prtF28) (Ruijter et al., 1999). Instead of fwnA1, this strain has two other mutations ( $\triangle \arg B$ and pyrA6 - used as transformation markers), making it an arginine and uridine auxotroph (Goosen et al., 1987; Lenouvel et al., 2002). When transforming NW186 with a gene of interest, one of the two auxotrophies, in our case the uridine auxotrophy, is alleviated, and transformants can be more easily selected for by growing $A$. niger on medium without uridine supplement. Thus, while both arginine and uridine have to be added to the parent strain, the transformants need only arginine as supplement in order to restore the growth phenotype.

However, in the experiment of which the results are presented in Figure 8.3, the $A$. niger cultures had grown very poorly in the first 18 hours before induction of FumT (under the control of the xylose inducible promoter $x \ln D$ (Peij et al., 1997)). We traced this back to not having added the correct supplements to the A. niger cultures, which were then added in retrospect. Thus, even though the initial data looked promising (Figure 8.4), the results could not be trusted, and the experiment was repeated. Surprisingly, at the time, fumarate production of the FumT transformants compared to the parent strain did not look as promising when grown with the correct supplementation (Figure 8.4).
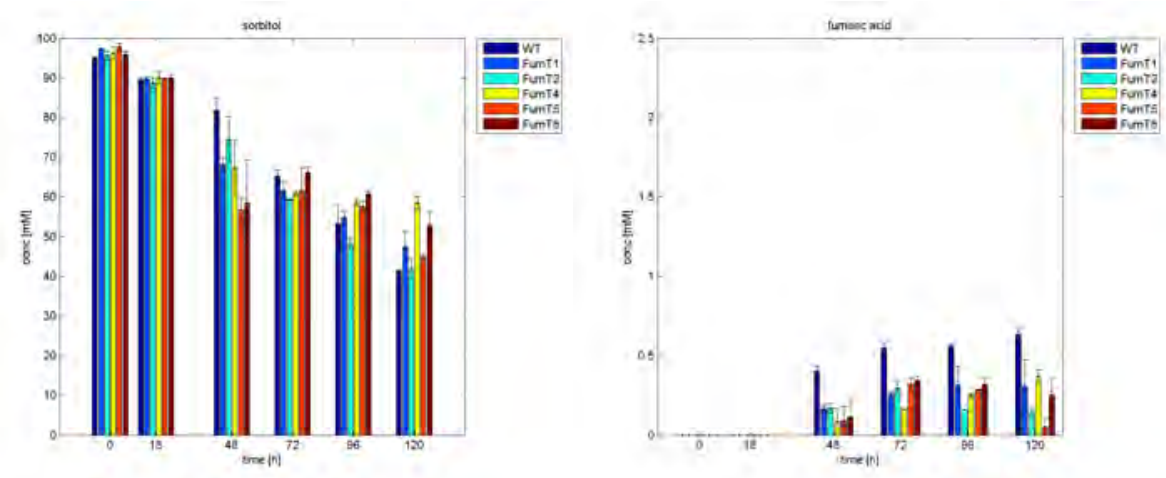

FIGURE 8.4.A. niger sorbitol consumption (left) and fumarate production (right) in repeated growth experiment. The second comparison of various $A$. niger FumT transporter mutants to the untransformed parent strain ("WT") looked less promising. 
Despite this setback, we reasoned that, although we did not understand how, the initial increase in fumarate secretion under arginine limitation could still be due to the presence of FumT in the transformants, as we had observed a clear difference in fumarate secretion in the FumT transformants compared to the parent strain ("WT", Figure 8.3). However, the untransformed strain used as WT in Figure 8.3 still had both transformation markers ( $\triangle \arg B$ and $p y r A 6$ ), and thus required both the addition of arginine and uridine in retrospect. Comparison of transformants to this strain is a valid approach when screening for increased production of a compound of interest, and had been applied successfully in our lab, e. g. by van der Straat et al. (2014). However, comparing strains with different supplemental requirements meant that this control could not unambiguously answer the question whether the effect observed in the initial experiment was due to the presence of FumT in A. niger. Therefore, a new control was introduced, in which uridine prototrophy is restored by transforming the strain with "the plasmid pGW635 (Goosen et al., 1989), carrying the pyrA gene of $A$. niger" (from Chapters 3 and 4).

To summarise a multitude of follow-up experiments in which we tried to reproduce our findings presented in Figure 8.3: Eventually, we concluded that heterologous expression of FumT did not lead to increased fumarate secretion in A. niger, and that our initial observation had been a false positive. Trying to figure out why the results of our initial experiment were not reproducible was a crucial point for two reasons: First, we started to transition towards a more chemically defined medium, and thus an increased understanding of the system we were working with, by avoiding the use of yeast extract, which had allowed auxotrophic $A$. niger strains to grow, albeit poorly, even in the absence of the timely addition of the correct supplements. Second, we observed that not adding arginine to the medium always led to the same result of increased $A$. niger fumarate secretion. And it was this recurrence of our initial observation that made me curious about finding out what was actually going on in the A. niger $\triangle a r g B$ mutants, marking the first change of how I approached the complex issue of fungal organic acid production.

Rather than focussing on identifying a single key component of fumarate production, i.e. the $R$. delemar fumarate exporter, I wanted to gain a more comprehensive understanding of fungal fumarate production, and started exploring the initial RNA seq and proteomics data obtained from the $R$. delemar high- and low-fumarate producing conditions in a more systematic way. It became apparent that fumarate is an intermediate in a much larger number of pathways than I had initially anticipated, as the only pathways deemed relevant for biotechnolog- 
ical production of fumarate were the oxidative and reductive routes of the TCA cycles (Engel et al., 2008; Straathof and van Gulik, 2012). Most interestingly, fumarate is a by-product in arginine biosynthesis, offering an explanation for why we observed increased fumarate secretion when the $A$. niger arginine "auxotroph" $(\triangle \arg B)$ was not supplemented with arginine, but was forced to biosynthesise arginine from an unknown constituent of yeast extract. These insights led to the holistic overview of the pathways involved in fumarate production, described in Chapter 2, and we could now explain why a high C:N ratio was key for $R$. delemar fumarate accumulation.

\subsection{Fumarate production in A. niger}

Having gained a better understanding of the network underlying fumarate accumulation in the natural fumarate producer $R$. delemar, we wanted to use our new insights for a second attempt at converting $A$. niger into a cell-factory for biotechnological fumarate production. Exploiting the urea ${ }^{2}$ cycle, which is interrupted in the $A$. niger $\triangle \arg B$ mutants (Figure 8.5), seemed a promising approach. Our rationale was based on both the analyses of the $R$. delemar -omics data, from which amino acid catabolism and the inferred resultant increase in metabolic fluxes through the urea cycle had emerged as a crucial mechanism contributing to the accumulation of fumarate under nitrogen limited conditions (Chapter 2), and our initial observations in the arginine auxotrophic A. niger mutant (Figure 8.3): We hypothesised that our observations were a result of forcing the $A$. niger $\triangle \arg B$ mutant to biosynthesise arginine from yeast extract. From this, we speculated that adding citrulline instead of arginine would lead to a more controlled increase in extracellular fumarate accumulation as a result of supplying a defined precursor for arginine biosynthesis (Figure 8.5).

However, supplementing the A. niger $\triangle \arg B$ mutant with citrulline instead of arginine did not lead to the expected increase in fumarate secretion. Instead, we observed increased citrate secretion. Similarly, we observed increased citrate instead of fumarate secretion when emulating the culture conditions that triggered R. delemar fumarate accumulation (Engel et al., 2008; Foster and Waksman, 1939b; Straathof and van Gulik, 2012). Mechanistically, I attributed the increase in extracellular citrate instead of fumarate accumulation to the possibility that any increase in intracellular fumarate was directly succeeded by conversion of fumarate to malate via the A. niger cytosolic fumarase, and then anti-port of

\footnotetext{
${ }^{2}$ While the enzymes are the same in fungi and mammals, it is not clear whether fungi actually produce urea as end-product, and I referred to it as "ornithine cycle" in later chapters.
} 

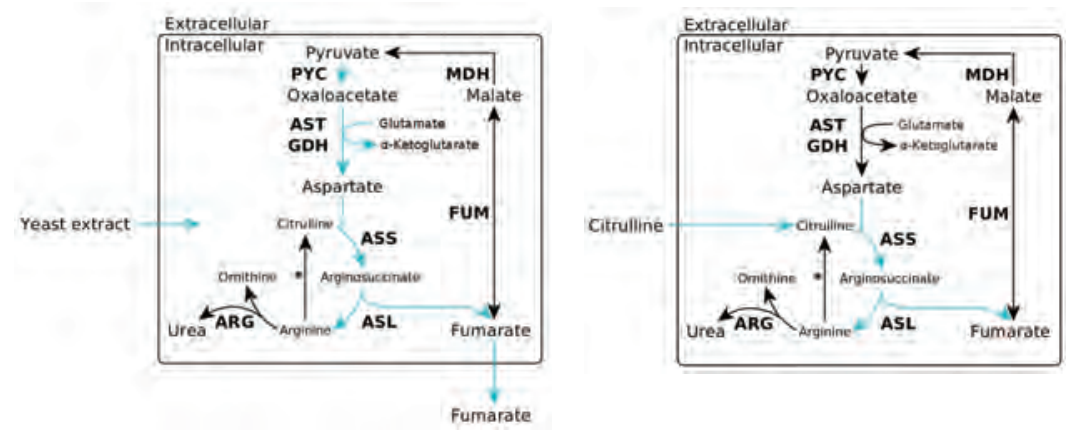

FIGURE 8.5. Turning $A$. niger into a cell-factory for fumarate production. Exploiting the urea cycle in the $A$. niger $\triangle \arg B$ mutant. $\mathrm{PYC}=$ pyruvate carboxylase, $\mathrm{AST}=$ aspartate amino-transferase, $\mathrm{GDH}=$ glutamate dehyrogenase, $\mathrm{ASS}=$ argininosuccinate synthase, $\mathrm{ASL}=$ argininosuccinate lyase, $\mathrm{ARG}=$ arginase, $\mathrm{FUM}=$ fumarase, $\mathrm{MDH}=$ malate dehydrogenase, ${ }^{*}=$ nitric-oxide synthase (putative).

malate and citrate across the mitochondrial membrane (Figure 8.6). This was consistent with the finding that $A$. niger citrate secretion is preceded by intracellular malate accumulation (Karaffa and Kubicek, 2003), and that expressing the $R$. delemar fum $R$ gene in $A$. niger had been a successful approach to increase citrate secretion in this fungus (De Jongh and Nielsen, 2008).

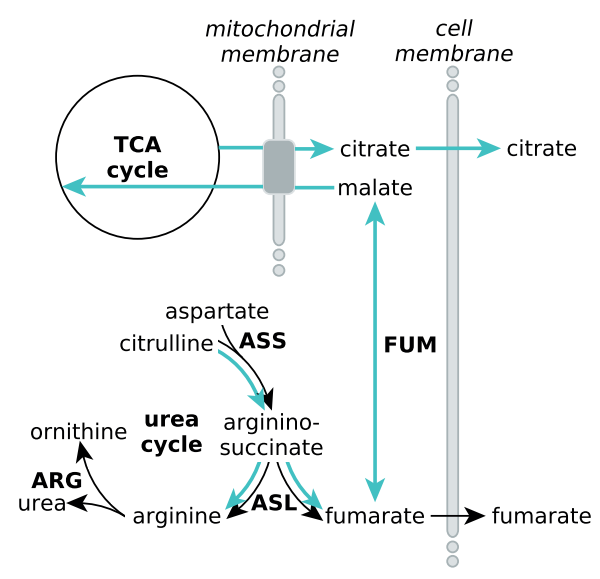

FIGURE 8.6. A. niger organic acid production via the urea cycle. Instead of increased accumulation of extracellular fumarate, we consistently observed altered citrate accumulation instead. Based on literature, we attributed this to the conversion of fumarate to malate, and subsequent malate-citrate antiport over the mitochondrial membrane. FUM = fumarase, $\mathrm{ASS}=$ argininosuccinate synthase, $\mathrm{ASL}=$ argininosuccinate lyase, $\mathrm{ARG}=$ arginase. 


\subsection{Cutting the Gordian knot of $\boldsymbol{A}$. niger citrate secretion}

The seemingly insurmountable challenge of re-routing $A$. niger metabolism towards increased fumarate secretion led to (multiple failed) attempts to create an $A$. niger fumarase $\mathrm{KO}$ mutant, as well as the setup of a transcriptomics experiment aimed at clarifying why $A$. niger citrate secretion increased upon supplementing the $\triangle \arg B$ mutant with citrulline rather than arginine (leading to the comparative transcriptomics analyses described in Chapter 4). Meanwhile, an unrelated trancriptomic experiment in an A. niger pyruvate carboxylase (PYC) overexpression mutant, which had shown increased citrate production compared to the parent strain (also NW186), provided further insights into the biological relevance of $A$. niger citrate secretion: The goal of the PYC transcriptomics experiment was to identify putative citrate exporter candidates. However, now being more sceptical in identifying specific organic acid exporters based on the fold-changes in only two contrasting conditions ${ }^{3}$, I was hesitant in pinpointing the single most promising citrate exporter candidate from the multitude of possible candidates, not least because I had no clear idea about what type of transporter we were actually looking for.

However, a noticeable observation in the PYC overexpression experiment was the enrichment of transporter-type proteins that were associated to compounds called "siderophores" (Greek: iron carrier). Since citrate has a natural ability to chelate iron, and in fact consuming citrus fruit enhances iron absorption in the human body, I began digging further into the nature of iron siderophores, which are in effect iron scavengers for microorganisms (Neilands, 1995). This eventually led to the hypothesis that citrate might act as iron siderophores in the A. niger NW186 $\triangle \arg B$ mutant, a hypothesis that was further explored and validated experimentally (Chapter 3). Importantly, drawing these connections marked the second change of how I dealt with the complexity of fungal organic acid production, reflected in the recurring sentence of Chapters 2 and 3:

"[Although] the [culture] conditions [that increase fumarate yields/required for A. niger citrate overproduction] are well [established/described], <Chapter 2/Chapter 3>"

<Chapter 2>: "[...] the network underlying the accumulation of fumarate is not yet fully understood."

<Chapter 3>: "[...] but the physiological reasons underlying extracellular citrate accumulation are not yet fully understood."

\footnotetext{
${ }^{3}$ Note that "contrasting conditions" here refer to the comparison of the PYC mutant to the parent strain (also NW186).
} 
Thus, while I had initially transitioned from pinpointing a single key element of $R$. delemar fumarate secretion to understanding how fumarate was produced in the first place, I now had transitioned into trying to unravel why our $A$. niger gluconate and oxalate non-producing $\triangle \arg B$ mutant secreted citrate under conditions which were detrimental to citrate secretion in wild type A. niger.

The potential of looking at the fungus not only as isolated cell-factory, but also in the context of its environment, is illustrated in the comparison of different mechanisms underlying increased $A$. niger citrate secretion, described in Chapters 3 and 4: While we can evoke increased citrate productivity by forcing the fungus into overflow metabolism, in our case by adding excess amounts of citrulline supplement (Chapter 4), citrate yield is more effectively increased by prompting a certain degree of cooperation, $i$. e. applying adaptive pressure (Chapter 3, data re-used for comparison in Chapter 4).

Thus, in the case of iron-limitation, increased citrate secretion is a means for the fungus to scavenge iron (Figure 8.7 (top)), whereas we attribute increased $A$. niger citrate secretion to overflow metabolism due to excess NADH generation in the case in which excess citrulline is added to the medium (Figure 8.7 (middle)). In the broadest definition, metabolic overflow describes the uncoupling of anabolism from catabolism under conditions of carbon excess. More specifically, overflow metabolism is the situation in which the rate of glycolysis is too high for other biosynthetic processes (anabolism) to keep up. The TCA cycle plays a crucial role to restore the metabolic imbalances, as high glycolytic flux will lead to a large influx of carbon into the TCA cycle. Since the TCA cycle itself cannot act as carbon sink, TCA cycle intermediates have to leave the cycle (Owen et al., 2002). Under conditions of balanced growth, these intermediates provide precursors for anabolic reactions. However, under conditions of too high influx, carbon is often secreted and "lost"; these compounds are termed overflow metabolites. Continued functioning of the TCA cycle is thus ensured by a balance between cataplerosis, i.e. the influx of TCA cyle metabolites, and anaplerosis, i.e. the efflux of TCA cycle intermediates.

Another consequence of carbon excess is that this will lead to an imbalance of carbon to other elemental biomass precursors, such as nitrogen and/or phosphate. As described in Chapter 2, we connected accumulation of fumarate under nitrogen limitation to increased amino-acid turnover via the urea cycle, yielding fumarate as by-product. However, in contrast to the effect of nitrogen limitation on fumarate secretion in $R$. delemar, reports on the effect of nitrogen limitation on $A$. niger organic acid production are not as unified. Studies on citrate production in $A$. niger showed that phosphate exhaustion is the crucial factor in the 
shift from the growth phase to the (organic acid) production phase, which was observed even when nitrogen was not limiting in the culture medium (Kubicek and Röhr, 1977). However, under phosphate-limiting conditions, the citrate yield is inversely correlated to the nitrogen content of the culture medium (Kubicek and Röhr, 1977).
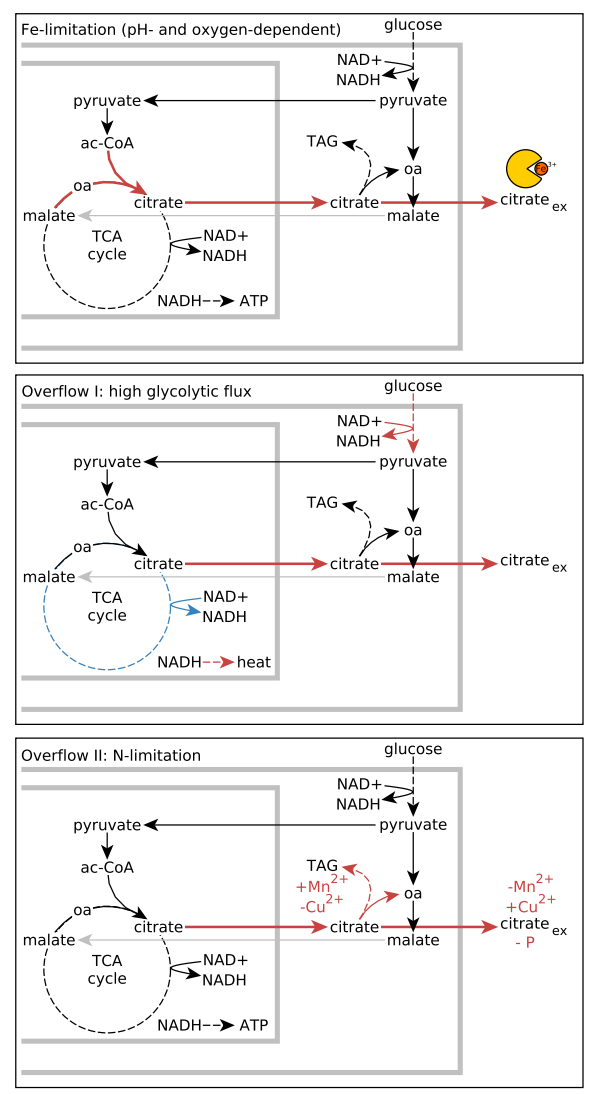

FIGURE 8.7.A. niger citrate production under different conditions. Besides the two mechanisms underlying $A$. niger citrate accumulation described in Chapters 3 and 4, there is another type of citrate "overflow" under N-limitation (bottom): In the presence of a high $\mathrm{C}: \mathrm{N}$ ratio, $A$. niger shifts metabolism from growth to the production of storage lipids (TAG = triacylglycerides), which, depending on other factors such as the presence or absence of certain trace metals and $\mathrm{P}$ in the culture medium, results in citrate overflow instead.

The effect of a high C:N ratio has also been studied in various oleaginous yeasts, which are able to overproduce citrate under certain conditions (Granger et al., 1993). In the yeasts studied, carbon and nitrogen are directed towards 
the formation of biomass until nitrogen "runs out", and the remaining carbon is directed towards the accumulation of storage lipids, most prominently tryacylglycerides (TAGs). Citrate, when leaving the TCA cycle, acts as direct precursor for TGA biosynthesis via ATP-citrate lyase (Acl, EC 2.3.3.8), and it has been found that flux through Acl was increased under nitrogen limited conditions in the citrate producer Yarrowia lipolytica, leading to excess TAG accumulation, and thus lower citrate secretion (Zhang et al., 2016).

We found that expression of $a c l$ was not correlated to higher or lower citrate secretion in our experimental setups described in Chapters 3 and 4. This agrees with previous reports that $A$. niger acl expression is not a marker for high or low citrate secretion in this fungus: Under citrate producing conditions, acl gene expression in A. niger H915-1 has been found to be up-regulated (Yin et al., 2017). In another instance, the enzyme itself became undetectable during citrate production in a citrate producing $A$. niger strain Yang No.2, while it was present in high levels in an oxalate producing A. niger strain SL1 (Leangon et al., 2000). These observations are in line with the hypothesis that A. niger Acl is subject to fine rather than coarse regulation, and as such is also not regulated by the amount of nitrogen in the medium (Pfitzner et al., 1987).

However, we found that fatty acid biosynthesis was down-regulated in the conditions in which more citrate was produced in both the iron and the supplement experiment. Although not as clearly connected to nitrogen exhaustion as in yeasts (Goldberg et al., 2006), there has been considerable evidence suggesting that $A$. niger lipid biosynthesis and citrate accumulation are mutually exclusive processes, and as such are affected inversely by different medium components (Figure 8.7 (bottom)). For example, it has been found that manganese (Mn) deficiency, the condition coinciding with increased citrate secretion (Kubicek and Röhr, 1977), leads to significantly lower lipid levels (in mg/g mycelial dry weight) in every $A$. niger strain tested, and the difference was most evident for TAGs (Orthofer et al., 1979). On the other hand, addition of copper $(\mathrm{Cu})$ ions to culture medium containing manganese antagonises the effect of manganese induced citrate inhibition, and induces increased citrate secretion (Kubicek and Röhr, 1977), a situation in which the levels of total lipids are reduced (Jernejc and Legiša, 2002).

The contrasting effects of manganese and copper ions on A. niger citrate secretion can be traced back to the requirement of malic enzyme (EC 1.1.1.40) and cytosolic isocitrate dehydrogenase (EC 1.1.1.42) for metal ion co-factors (Jernejc and Legiša, 2002; Meixner-Monori et al., 1986). These enzymes could be responsible for the production of a major portion of the NADPH necessary 
for lipid biosynthesis. While $\mathrm{Mn}^{2+}$ and/or $\mathrm{Mg}^{2+}$ activate these enzymes, $\mathrm{Cu}^{2+}$ competitively inhibits the binding site and, in the case of malic enzyme, abolishes its activity completely (Jernejc and Legiša, 2002). Thus, in the absence of $\mathrm{Mn}^{2+}$, or the presence of $\mathrm{Cu}^{2+}$, not enough NADPH is produced, and lipid biosynthesis cannot occur, probably leading to increased citrate secretion, as depicted in Figure 8.7 (bottom). In this context, it has to be noted that $A$. niger metabolism grown with or without $\mathrm{Mn}$ added to the medium is almost the same until both nitrogen and phosphate are exhausted (Kubicek and Röhr, 1977). It is therefore likely that phosphate limitation affects citrate production not only because the repeated building block of the DNA backbone contains a phosphate group, and phosphate limitation would therefore impair growth, but also because phosphate is a constituent of NADPH and ATP, both of which are required for lipid biosynthesis.

Finally, understanding the role of citrate under different culture conditions, and combining data from two alternative citrate producing conditions to condense a shortlist of the most promising citrate tranporter candidates, led to the successful identification of an A. niger citrate exporter, which was validated by heterologous expression in S. cerevisiae (Chapter 4).

\subsection{Identification of $\boldsymbol{A}$. niger sugar importers}

In parallel, a combination of wet- and dry-lab approaches led to the identification of glucose, xylose and rhamnose transporters (Chapters 5, 6 and 7). The mismatch between the number of identified sugar importers and the number of identified organic acid exporters reflects the mismatch between the already available knowledge about sugar importers and organic acid exporters. As the largest sub-family of the major facilitator superfamily, the structure of sugar importers (also sugar porters, SP) is well described (Pao et al., 1998). When performing differential proteomics analyses on plasmalemmal proteins isolated from A. niger grown with various different sugar sources and concentrations, I was therefore not forced to pinpoint likely candidates based on abundance fold-changes alone. Instead, I could already narrow down the list by discarding transporter proteins that did not fit the SP architecture.

The benefit of being able to fall back on already characterised transporter proteins for the identification of A. niger SPs was most obvious in the construction and use of profile hidden Markov models (HMMs). As described in Chapter 5, the SP family has a large number of identified members (Pao et al., 1998), providing a good basis for constructing profile HMMs. Most importantly though, 
there were already a number of biochemically characterised glucose and xylose transporters, from which more specific HMMs could be built. In contrast, there are few identified and biochemically characterised organic acid transporters, of which most are prokaryotic or mitochondrial. This was also found back in the results obtained when querying the $A$. niger ATCC 1015 in silico proteome with a citrate specific HMM; all the hits with the HMM were with proteins that were predicted to be either mitochondrial or peroxisomal (see Supplementary file 4 in Chapter 4). The transporter that was eventually identified and verified as $A$. niger citrate exporter has a typical MFS transporter structure with 12 transmembrane helix structures, and could thus not have been identified based on the transporter architecture alone, either.

\subsection{Transporter validation}

Another aspect to consider is the validation of transporter protein candidates in the context of the necessity for a microorganism to consume carbon for its own growth and survival $v s$ secreting it as the product we are interested in. Verification of the identified glucose and xylose transporter candidates (Chapters 5 and 6) was facilitated by the existence of a Saccharomyces cerevisiae hexose transporter null mutant (Wieczorke et al., 1999), which, for experimental validation of the xylose transporters, was engineered to metabolise xylose (Hector et al., 2011). In this mutant strain, heterologous expression of the identified glucose and xylose transporter candidates opens a new door, and it is in the interest of the host organism to make use of this entry to obtain the carbon that is available.

In contrast, heterologous expression of a given exporter protein candidate can, but need not, evoke cooperation of the host organism, and will therefore not necessarily result in increased secretion of the product of interest. Disregarding the cases in which the compound is not biosynthesised in the first place, which is less of an issue when dealing with metabolites of central carbon metabolism, this compound might still not be secreted in higher amounts, because it is either not synthesised in the correct compartment, or directly further metabolised rather than secreted. Thus, a positive result can be trusted, whereas negative results give no clear answers.

One option is to validate the identified exporter in the organism of its origin. In this regard, full KO mutants of a given substrate transporter, such as the $S$. cerevisiae hexose transporter mutant (Wieczorke et al., 1999), would be a powerful validation tool. However, this would imply that, for each biotechnological work-horse of choice, new full transporter KO mutants would have to be cre- 
ated separately. Additionally, this time-intensive and laborious process would be hampered by the lack of knowledge and identification of other possible exporter candidates that might either be specialised alternatives, or moonlighting of the product of interest. In addition, even if the compound of interest is partially present in the correct compartment out of which we want to transport it, this would still not solve the problem that the product of interest is not biosynthesised beyond the threshold for the transporter to show any additional effect. In this sense, transporter and pathway metabolic engineering are more effectively applied in tandem, as shown by van der Straat et al. (2014).

\subsection{Future outlook}

The issue that remains unresolved by the exporter validation approaches described above, and which has to be equally addressed in metabolic engineering approaches in general, is that the microorganism that we want to use as biotechnological cell-factory has to actually biosynthesise a certain amount of the product of interest. In this regard, the more classical approach of overexpressing genes encoding enzymes that convert a given substrate to a product of interest has been successful, but, in the case of $A$. niger, often seems a gamble whether it would work or not (Ratledge, 2000; Ruijter, 2000; Ruijter et al., 2000). Thus, one option I propose is to look at the issue from a different angle, which is to first understand the biological role of the product we are interested in, and then tailor our metabolic engineering towards increased dependency of our cell-factory on secreting this compound for its own survival. In this context, the discovery of Ruijter et al. (1999) unraveled an important principle of A. niger organic acid production: the researchers found that an $A$. niger mutant, which could no longer produce gluconate and oxalate due to mutations in glucose oxidase (GoxC) and oxaloacetate hydrolase (OahA), secreted citrate under conditions that used to be detrimental for extracellular citrate accumulation in favour of the other two organic acids. This finding is noteworthy not only because of the usefulness of the resultant strain, which is the basis of $A$. niger NW186 used for various studies throughout this thesis, but also because their work showed the effects of a very fundamental principle I consider crucial for future metabolic engineering approaches: As described in Chapter 3, I think that the reason these mutations led to increased citrate production was because citrate now was taking over the biological role of oxalate to increase bioavailability of iron. Iron limitation is only one of the culture conditions that favour increased citrate production, and most of the other culture conditions can be explained mechanistically, also summarised 
in Figure 8.7. However, the culture conditions that seem to have an influence on citrate production mechanistically, e.g. the external $\mathrm{pH}$ or the presence of $\mathrm{Mn}^{2+}$ ions, show less effect when the fungus has no other choice but to secrete citrate for its own growth and survival (Ruijter et al., 1999).

However, in most of the cases, the extracellular function of a given product of interest might not be known, or there simply might not be an extracellular function for this compound. A complementary approach to studying the potential cell-factory in the context of its environment is thus the use of genome-scale metabolic models (GSMs) for optimal metabolic engineering approaches. In GSMs, growth can also be coupled to product formation by algorithms such as OptKnock (Burgard et al., 2003) and RobustKnock (Tepper and Shlomi, 2009), which try to identify $\mathrm{KO}$ strategies that couple biomass formation to the production of the compound of interest. The use of GSMs is powerful especially if analysis of the the differences between in silico predictions and in vivo observations leads to a deeper understanding of complex biological mechanisms, providing a better knowledge base for the organisms under study in the form of updated GSMs.

GSMs were applied as a knowledge base to increase fumarate production in Torulopsis glabrata (Chen et al., 2015) and S. cerevisiae (Xu et al., 2012b). In our case, however, correct predictions of the observed phenotypes were hampered by the lack of restricted transport across the compartments in the A. niger GSM (Andersen et al., 2008). The importance of correct compartmentalisation predictions is illustrated in the example of overexpression of the cytosolic $R$. delemar fumR in $A$. niger, which lead to increased citrate production (De Jongh and Nielsen, 2008). The activity of FumR is inhibited by fumarate concentrations $\geq 2 \mathrm{mM}$ (Song et al., 2011), leading to secretion of excess fumarate in $R$. delemar. In A. niger, heterologous expression of FumR resulted in increased citrate production. According to the proposed mechanism depicted in Figure 8.6, this can be attributed to the conversion of fumarate to malate with the subsequent anti-port of malate and citrate across the mitochondrial membrane. In this, the lack of FumR inhibition implies that cytosolic fumarate concentrations in A. niger never reach the same levels as in $R$. delemar, suggesting fundamental differences in the distributions of enzymes and thus metabolites across the cell-organelles of these two filamentous fungi.

Another aspect relevant for this work is that GSMs do not take metabolic regulation into account, which is a problem in the context of the stringent control of central carbon metabolism. As an example, forcing increased flux through PFK might result in higher citrate secretion in silico, but in vivo, only overexpression of a truncated (due to post-translational modification (Mesojednik and Legiša, 2005; 
Mlakar and Legiša, 2006)) version of PFK, which was not subject to repression by citrate, has so far been successful (Capuder et al., 2009). Similarly, co-factor requirements of metalloproteins are not taken into account with GSMs, allthough even a GSM including all co-factor requirements of a given organism would not be able to predict the need for the organism to secrete citrate to scavenge iron. On the other hand, dynamic models can take metabolic regulation into account, as an example the modular dynamic model of the response of yeast to osmotic stress, created by Klipp et al. (2005). However, although this can provide a starting point for further optimisation of the model in an iterative cycle with experimental validation, it seems unlikely that a dynamic model will ever encompass the full complexity of an organism on genome-scale.

The lack of a clear biological role for fumarate secretion, or in any case the knowledge thereof, is still a bottleneck in converting $A$. niger into an efficient cell-factory for fumarate production. Intracellularly, fumarate might have an important role in post-transcriptional control, as it can modify proteins by attaching to a thiol group of a cysteine, thereby forming S-(2-succinyl) cysteine ("protein succination"). However, extracellular fumarate accumulation by $R$. delemar happens after the growth phase, and specifically when the nitrogen in the medium is depleted. As described in Chapter 2, this could be a result of amino acid degradation as a form of nitrogen turn-over, yielding fumarate as a by-product. Fumarate, in this mechanism, is regarded as a "waste-product", and secreted. To further unravel the biological role of fumarate for $R$. delemar, the fungus could be studied in the context of its environment and specifically its pathogenicity, as this can give information not only about the fungus as isolated entity, but also the reaction of the host it is infecting, thereby maybe giving insights into the role of the secreted products.

\subsection{Concluding remark}

In this thesis, the transition from identification to understanding implied a shift from applied to more fundamental insights concerning fungal organic acid production, especially with regard to $A$. niger citrate production and secretion. Although this can be translated into industrial applications, and we provided a number of tools to improve A. niger as biotechnological cell-factory, I think that research in academia should first and foremost focus on the pursuit of knowledge. In short, academia should be the playground for the curious and critical. 


\section{BIBLIOGRAPHY}

Abe, Ayumi, Oda, Yuji, Asano, Kozo, and Sone, Teruo.

"Rhizopus delemar is the proper name for Rhizopus oryzae fumaric-malic acid producers."

Mycologia 99.5 (2007): 714-722.

Abe, Ayumi, Sone, Teruo, Sujaya, I Nengah, Saito, Katsuichi, Oda, Yuji, Asano, Kozo, and Tomita, Fusao.

"rDNA ITS sequence of Rhizopus oryzae: its application to classification and identification of lactic acid producers."

Bioscience, biotechnology, and biochemistry 67.8 (2003): 1725-1731.

Ademark, Pia, de Vries, Ronald P, Hägglund, Per, Stålbrand, Henrik, and Visser, Jaap.

"Cloning and characterization of Aspergillus niger genes encoding an $\alpha$-galactosidase and a $\beta$-mannosidase involved in galactomannan degradation."

The FEBS Journal 268.10 (2001): 2982-2990.

Alazi, Ebru, Niu, Jing, Kowalczyk, Joanna E, Peng, Mao, Aguilar Pontes, Maria Victoria, Kan, Jan AL, Visser, Jaap, Vries, Ronald P, and Ram, Arthur FJ.

"The transcriptional activator GaaR of Aspergillus niger is required for release and utilization of d-galacturonic acid from pectin." FEBS letters 590.12 (2016): 1804-1815.

Alvarez-Vasquez, Fernando, González-Alcón, Carlos, and Torres, Néstor V.

"Metabolism of citric acid production by Aspergillus niger: Model definition, steady-state analysis and constrained optimization of citric acid production rate."

Biotechnology and Bioengineering 70.1 (2000): 82-108.

Andersen, Mikael R, Lehmann, Linda, and Nielsen, Jens.

"Systemic analysis of the response of Aspergillus niger to ambient $\mathrm{pH}$."

Genome biology 10.5 (2009): R47.

Andersen, Mikael R, Salazar, Margarita P, Schaap, Peter J, van de Vondervoort, Peter JI, Culley, David, Thykaer, Jette, Frisvad, Jens C, Nielsen, Kristian F, Albang, Richard, Albermann, Kaj, et al.

"Comparative genomics of citric-acid-producing Aspergillus niger ATCC 1015 versus enzyme-producing CBS 513.88."

Genome research 21.6 (2011): 885-897.

Andersen, Mikael Rørdam, Nielsen, Michael Lynge, and Nielsen, Jens.

"Metabolic model integration of the bibliome, genome, metabolome and reactome of Aspergillus niger."

Molecular Systems Biology 4.1 (2008): 178.

Arnaud, Martha B, Cerqueira, Gustavo C, Inglis, Diane O, Skrzypek, Marek S, Binkley, Jonathan, Chibucos, Marcus C, Crabtree, Jonathan, Howarth, Clinton, Orvis, Joshua, Shah, Prachi, et al.

"The Aspergillus Genome Database (AspGD): recent developments in comprehensive multispecies curation, comparative genomics and community resources." Nucleic acids research 40.D1 (2011): D653-D659.

Arvidsson, Samuel, Kwasniewski, Miroslaw, Riaño-Pachón, Diego Mauricio, and Mueller-Roeber, Bernd. "QuantPrime-a flexible tool for reliable high-throughput primer design for quantitative PCR." BMC bioinformatics 9.1 (2008): 465.

Battaglia, Evy, Hansen, Sara Fasmer, Leendertse, Anne, Madrid, Susan, Mulder, Harm, Nikolaev, Igor, and de Vries, Ronald P. "Regulation of pentose utilisation by AraR, but not XlnR, differs in Aspergillus nidulans and Aspergillus niger." Applied microbiology and biotechnology 91.2 (2011): 387.

Becker, Jessica and Boles, Eckhard.

"A modified Saccharomyces cerevisiae strain that consumes L-arabinose and produces ethanol." Applied and Environmental Microbiology 69.7 (2003): 4144-4150.

Behera, Shuvashish, Singh, Richa, Arora, Richa, Sharma, Nilesh Kumar, Shukla, Madhulika, and Kumar, Sachin. "Scope of algae as third generation biofuels."

Frontiers in bioengineering and biotechnology 2.

Benz, J Philipp, Protzko, Ryan J, Andrich, Jonas MS, Bauer, Stefan, Dueber, John E, and Somerville, Chris R. "Identification and characterization of a galacturonic acid transporter from Neurospora crassa and its application for Saccharomyces cerevisiae fermentation processes."

Biotechnology for biofuels 7.1 (2014): 20.

Bischof, Robert, Fourtis, Lukas, Limbeck, Andreas, Gamauf, Christian, Seiboth, Bernhard, and Kubicek, Christian P. 
"Comparative analysis of the Trichoderma reesei transcriptome during growth on the cellulase inducing substrates wheat straw and lactose."

Biotechnology for biofuels 6.1 (2013): 127.

Blatzer, Michael, Barker, Bridget M, Willger, Sven D, Beckmann, Nicola, Blosser, Sara J, Cornish, Elizabeth J, Mazurie, Aurelien, Grahl, Nora, Haas, Hubertus, and Cramer, Robert A.

"SREBP coordinates iron and ergosterol homeostasis to mediate triazole drug and hypoxia responses in the human fungal pathogen Aspergillus fumigatus."

PLoS genetics 7.12 (2011): e1002374.

Boeke, Jef D, Trueheart, Joshua, Natsoulis, Georges, and Fink, Gerald R.

"[10] 5-Fluoroorotic acid as a selective agent in yeast molecular genetics."

Methods in enzymology 154: 164-175.

Bolger, Anthony M, Lohse, Marc, and Usadel, Bjoern.

"Trimmomatic: a flexible trimmer for Illumina sequence data."

Bioinformatics 30.15 (2014): 2114-2120.

Bos, CJ, Debets, AJM, Swart, K, Huybers, A, Kobus, G, and Slakhorst, SM.

"Genetic analysis and the construction of master strains for assignment of genes to six linkage groups in Aspergillus niger."

Current genetics 14.5 (1988): 437-443.

Burgard, Anthony P, Pharkya, Priti, and Maranas, Costas D.

"Optknock: a bilevel programming framework for identifying gene knockout strategies for microbial strain optimization."

Biotechnology and bioengineering 84.6 (2003): 647-657.

Cabezon, Virginia, Llama-Palacios, Arancha, Nombela, César, Monteoliva, Lucía, and Gil, Concha.

"Analysis of Candida albicans plasma membrane proteome."

Proteomics 9.20 (2009): 4770-4786.

Cao, Ningjun, Du, Jianxin, Gong, CS, and Tsao, GT.

"Simultaneous Production and Recovery of Fumaric Acid from Immobilized Rhizopus oryzae with a Rotary Biofilm Contactor and an Adsorption Column."

Applied and environmental microbiology 62.8 (1996): 2926-2931.

Capuder, Maja, Šolar, Tina, Benčina, Mojca, and Legiša, Matic.

"Highly active, citrate inhibition resistant form of Aspergillus niger 6-phosphofructo-1-kinase encoded by a modified pfkA gene." Journal of biotechnology 144.1 (2009): 51-57.

Carvalho, Neuza DSP, Arentshorst, Mark, Jin Kwon, Min, Meyer, Vera, and Ram, Arthur FJ.

"Expanding the ku70 toolbox for filamentous fungi: establishment of complementation vectors and recipient strains for advanced gene analyses."

Applied microbiology and biotechnology 87.4 (2010): 1463-1473.

Cássio, FERNANDA and Leao, C.

"Low-and high-affinity transport systems for citric acid in the yeast Candida utilis." Applied and environmental microbiology 57.12 (1991): 3623-3628.

Chen, Xiulai, Wu, Jing, Song, Wei, Zhang, Limei, Wang, Hongjiang, and Liu, Liming.

"Fumaric acid production by Torulopsis glabrata: engineering the urea cycle and the purine nucleotide cycle."

Biotechnology and bioengineering 112.1 (2015): 156-167.

Chen, Xiulai, Zhu, Pan, and Liu, Liming.

"Modular optimization of multi-gene pathways for fumarate production."

Metabolic engineering 33: 76-85.

Chesters, CGC and Rolinson, GN.

"Zinc in the metabolism of a strain of Aspergillus niger."

Microbiology 5.3 (1951): 553-558.

Christensen, Mette, Borza, Tudor, Dandanell, Gert, Gilles, Anne-Marie, Barzu, Octavian, Kelln, Rod A, and Neuhard, Jan.

"Regulation of expression of the 2-deoxy-D-ribose utilization regulon, deoQKPX, from Salmonella enterica serovar typhimurium." Journal of bacteriology 185.20 (2003): 6042-6050.

Claudel-Renard, Clotilde, Chevalet, Claude, Faraut, Thomas, and Kahn, Daniel.

"Enzyme-specific profiles for genome annotation: PRIAM."

Nucleic acids research 31.22 (2003): 6633-6639.

Coelho, Marco A, Gonçalves, Carla, Sampaio, José Paulo, and Gonçalves, Paula.

"Extensive intra-kingdom horizontal gene transfer converging on a fungal fructose transporter gene."

PLoS genetics 9.6 (2013): e1003587.

Colabardini, Ana Cristina, Ries, Laure Nicolas Annick, Brown, Neil Andrew, Dos Reis, Thaila Fernanda, Savoldi, Marcela, Goldman, Maria Helena S, Menino, João Filipe, Rodrigues, Fernando, and Goldman, Gustavo Henrique.

"Functional characterization of a xylose transporter in Aspergillus nidulans."

Biotechnology for biofuels 7.1 (2014): 46.

Consortium, UniProt et al.

"UniProt: a hub for protein information."

Nucleic acids research : gku989.

Coons, DAVID M, Vagnoli, PAOLA, and Bisson, LINDA F. 
"The C-terminal Domain of Snf3p is Sufficient to Complement the Growth Defect of snf3 Null Mutations in Saccharomyces cervisiae: SNF3 Functions in Glucose Recognition."

Yeast 13.1 (1997): 9-20.

Cox, CHARLES D.

"Iron uptake with ferripyochelin and ferric citrate by Pseudomonas aeruginosa."

Journal of bacteriology 142.2 (1980): 581-587.

Cox, GB, Gibson, F, Luke, RKJ, Newton, NA, O'brien, IG, and Rosenberg, H.

"Mutations affecting iron transport in Escherichia coli."

Journal of bacteriology 104.1 (1970): 219-226.

Cox, Jürgen and Mann, Matthias.

"MaxQuant enables high peptide identification rates, individualized ppb-range mass accuracies and proteome-wide protein quantification."

Nature biotechnology 26.12 (2008): 1367-1372.

Cubero, Beatriz, Gómez, Dennis, and Scazzocchio, Claudio.

"Metabolite repression and inducer exclusion in the proline utilization gene cluster of Aspergillus nidulans."

Journal of bacteriology 182.1 (2000): 233-235.

Currie, James N.

"The citric acid fermentation of Aspergillus niger."

J Biol Chem 31: 15-37.

Davids, Mark, Hugenholtz, Floor, dos Santos, Vitor Martins, Smidt, Hauke, Kleerebezem, Michiel, and Schaap, Peter J.

"Functional profiling of unfamiliar microbial communities using a validated de novo assembly metatranscriptome pipeline."

PloS one 11.1 (2016): e0146423.

Davis, EO and Henderson, PJ.

"The cloning and DNA sequence of the gene $x y l E$ for xylose-proton symport in Escherichia coli K12."

Journal of Biological Chemistry 262.29 (1987): 13928-13932.

De Jongh, WA and Nielsen, Jens.

"Enhanced citrate production through gene insertion in Aspergillus niger."

Metabolic engineering 10.2 (2008): 87-96.

de Oliveira, José Miguel P Ferreira and de Graaff, Leo H.

"Proteomics of industrial fungi: trends and insights for biotechnology."

Applied microbiology and biotechnology 89.2 (2011): 225-237.

de Oliveira, José Miguel P Ferreira, van Passel, Mark WJ, Schaap, Peter J, and de Graaff, Leo H.

"Shotgun proteomics of Aspergillus niger microsomes upon D-xylose induction."

Applied and environmental microbiology 76.13 (2010): 4421-4429.

"Proteomic analysis of the secretory response of Aspergillus niger to D-maltose and D-xylose."

PLoS One 6.6 (2011): e20865.

de Souza, Wagner R, de Gouvea, Paula F, Savoldi, Marcela, Malavazi, Iran, de Souza Bernardes, Luciano A, Goldman, Maria Helena S, de Vries, Ronald P, de Castro Oliveira, Juliana V, and Goldman, Gustavo H.

"Transcriptome analysis of Aspergillus niger grown on sugarcane bagasse."

Biotechnology for biofuels 4.1 (2011): 40.

de Souza, Wagner Rodrigo, Maitan-Alfenas, Gabriela Piccolo, de Gouvêa, Paula Fagundes, Brown, Neil Andrew, Savoldi, Marcela, Battaglia, Evy, Goldman, Maria Helena S, de Vries, Ronald P, and Goldman, Gustavo Henrique.

"The influence of Aspergillus niger transcription factors AraR and XlnR in the gene expression during growth in D-xylose, Larabinose and steam-exploded sugarcane bagasse."

Fungal genetics and biology 60: 29-45.

de Vries, Ronald $\mathrm{P}$ and Visser, Jaap.

"Aspergillus enzymes involved in degradation of plant cell wall polysaccharides."

Microbiology and molecular biology reviews 65.4 (2001): 497-522.

de Vries, Ronald P, Visser, Jaap, and de Graaff, Leo H.

"CreA modulates the XlnR-induced expression on xylose of Aspergillus niger genes involved in xylan degradation."

Research in Microbiology 150.4 (1999): 281-285.

Delmas, Stéphane, Pullan, Steven T, Gaddipati, Sanyasi, Kokolski, Matthew, Malla, Sunir, Blythe, Martin J, Ibbett, Roger, Campbell, Maria, Liddell, Susan, Aboobaker, Aziz, et al.

"Uncovering the genome-wide transcriptional responses of the filamentous fungus Aspergillus niger to lignocellulose using RNA sequencing."

PLoS genetics 8.8 (2012): e1002875.

Delom, Frédéric, Szponarski, Wojciech, Sommerer, Nicolas, Boyer, Jean-Christophe, Bruneau, Jean-Michel, Rossignol, Michel, and Gibrat, Rémy.

"The plasma membrane proteome of Saccharomyces cerevisiae and its response to the antifungal calcofluor."

Proteomics 6.10 (2006): 3029-3039.

Deng, Dong, Xu, Chao, Sun, Pengcheng, Wu, Jianping, Yan, Chuangye, Hu, Mingxu, and Yan, Nieng.

"Crystal structure of the human glucose transporter GLUT1." 
Nature 510.7503 (2014): 121-125.

Derntl, Christian, Gudynaite-Savitch, Loreta, Calixte, Sophie, White, Theresa, Mach, Robert L, and Mach-Aigner, Astrid R.

"Mutation of the Xylanase regulator 1 causes a glucose blind hydrolase expressing phenotype in industrially used Trichoderma strains."

Biotechnology for biofuels 6.1 (2013): 62 .

Dhillon, Gurpreet Singh, Brar, Satinder Kaur, Verma, Mausam, and Tyagi, Rajeshwar Dayal.

"Utilization of different agro-industrial wastes for sustainable bioproduction of citric acid by Aspergillus niger."

Biochemical Engineering Journal 54.2 (2011): 83-92.

Dobin, Alexander, Davis, Carrie A, Schlesinger, Felix, Drenkow, Jorg, Zaleski, Chris, Jha, Sonali, Batut, Philippe, Chaisson, Mark, and Gingeras, Thomas R.

"STAR: ultrafast universal RNA-seq aligner."

Bioinformatics 29.1 (2013): 15-21.

dos Reis, Thaila Fernanda, Menino, João Filipe, Bom, Vinícius Leite Pedro, Brown, Neil Andrew, Colabardini, Ana Cristina, Savoldi, Marcela, Goldman, Maria Helena S, Rodrigues, Fernando, and Goldman, Gustavo Henrique.

"Identification of glucose transporters in Aspergillus nidulans."

PLoS One 8.11 (2013): e81412.

Du, Jing, Li, Sijin, and Zhao, Huimin.

"Discovery and characterization of novel d-xylose-specific transporters from Neurospora crassa and Pichia stipitis."

Molecular BioSystems 6.11 (2010): 2150-2156.

Dujon, Bernard, Sherman, David, Fischer, Gilles, Durrens, Pascal, Casaregola, Serge, Lafontaine, Ingrid, De Montigny, Jacky, Marck, Christian, Neuvéglise, Cécile, Talla, Emmanuel, et al.

"Genome evolution in yeasts."

Nature 430.6995 (2004): 35-44.

Dutton, Martin V and Evans, Christine S.

"Oxalate production by fungi: its role in pathogenicity and ecology in the soil environment."

Canadian journal of microbiology 42.9 (1996): 881-895.

Eddy, Sean R.

"Profile hidden Markov models."

Bioinformatics (Oxford, England) 14.9 (1998): 755-763.

Edwards, Meredith C and Doran-Peterson, Joy.

"Pectin-rich biomass as feedstock for fuel ethanol production."

Applied microbiology and biotechnology : 1-11.

Eisendle, Martin, Oberegger, Harald, Buttinger, Rudolf, Illmer, Paul, and Haas, Hubertus

"Biosynthesis and uptake of siderophores is controlled by the PacC-mediated ambient-pH regulatory system in Aspergillus nidulans."

Eukaryotic cell 3.2 (2004): 561-563.

Emiliani, E and Bekes, P.

"Enzymatic oxalate decarboxylation in Aspergillus niger."

Archives of Biochemistry and Biophysics 105.3 (1964): 488-493.

Engel, Carol A Roa, Straathof, Adrie JJ, Zijlmans, Tiemen W, van Gulik, Walter M, and van der Wielen, Luuk AM.

"Fumaric acid production by fermentation."

Applied microbiology and biotechnology 78.3 (2008): 379-389.

Fang, Ferric C and Casadevall, Arturo.

"Reductionistic and holistic science."

2011.

Farwick, Alexander, Bruder, Stefan, Schadeweg, Virginia, Oreb, Mislav, and Boles, Eckhard.

"Engineering of yeast hexose transporters to transport D-xylose without inhibition by D-glucose."

Proceedings of the National Academy of Sciences 111.14 (2014): 5159-5164.

Feillet, F and Leonard, JV.

"Alternative pathway therapy for urea cycle disorders."

Journal of inherited metabolic disease 21: 101-111.

Felig, Philip.

"The glucose-alanine cycle."

Metabolism 22.2 (1973): 179-207.

Ferreira, Danielly, Nobre, Alexandra, Silva, Marta Luisa, Faria-Oliveira, Fábio, Tulha, Joana, Ferreira, Célia, and Lucas, Cândida. "XYLH encodes a xylose $/ \mathrm{H}^{+}$symporter from the highly related yeast species Debaryomyces fabryi and Debaryomyces hansenii." FEMS yeast research 13.7 (2013): 585-596.

Finn, Robert D, Clements, Jody, and Eddy, Sean R. "HMMER web server: interactive sequence similarity searching." Nucleic acids research 39.suppl_2 (2011): W29-W37.

Forment, Josep V, Flipphi, Michel, Ramón, Daniel, Ventura, Luisa, and MacCabe, Andrew P "Identification of the $m s t E$ gene encoding a glucose-inducible, low affinity glucose transporter in Aspergillus nidulans." Journal of Biological Chemistry 281.13 (2006): 8339-8346. 
Forment, Josep V, Flipphi, Michel, Ventura, Luisa, González, Ramón, Ramón, Daniel, and MacCabe, Andrew P.

"High-affinity glucose transport in Aspergillus nidulans is mediated by the products of two related but differentially expressed genes."

PLoS One 9.4 (2014): e94662.

Foster, Jackson W and Waksman, Selman A.

"The production of fumaric acid by molds belonging to the genus Rhizopus."

Journal of the American Chemical Society 61.1 (1939a): 127-135.

"The specific effect of zinc and other heavy metals on the growth and nutrition of Rhizopus."

Journal of Bacteriology 37.6 (1939b): 599.

Foster, JW, Carson, SF, Anthony, DS, Davis, JB, Jefferson, WE, and Long, MV.

"Aerobic Formation of Fumaric Acid in the Mold Rhizopus Nigricans Synthesis by Direct C2 Condensation."

Proceedings of the National Academy of Sciences 35.12 (1949): 663-672.

Franken, Angelique CW, Lechner, Beatrix E, Werner, Ernst R, Haas, Hubertus, Lokman, B Christien, Ram, Arthur FJ, van den Hondel, Cees AMJJ, de Weert, Sandra, and Punt, Peter J.

"Genome mining and functional genomics for siderophore production in Aspergillus niger."

Briefings in functional genomics 13.6 (2014): 482-492.

Friedberg, D, Peleg, Y, Monsonego, A, Maissi, S, Battat, E, Rokem, JS, and Goldberg, I.

"The fumR gene encoding fumarase in the filamentous fungus Rhizopus oryzae: cloning, structure and expression."

Gene 163.1 (1995): 139-144.

Frost, GE and Rosenberg, H.

"The inducible citrate-dependent iron transport system in Escherichia coli K12."

Biochimica et Biophysica Acta (BBA)-Biomembranes 330.1 (1973): 90-101.

Futagami, Taiki, Mori, Kazuki, Yamashita, Ayaka, Wada, Shotaro, Kajiwara, Yasuhiro, Takashita, Hideharu, Omori, Toshiro, Takegawa, Kaoru, Tashiro, Kosuke, Kuhara, Satoru, et al.

"Genome sequence of the white koji mold Aspergillus kawachii IFO 4308, used for brewing the Japanese distilled spirit shochu." Eukaryotic cell 10.11 (2011): 1586-1587.

Gadd, Geoffrey M.

"Fungal production of citric and oxalic acid: importance in metal speciation, physiology and biogeochemical processes." Advances in microbial physiology 41: 47-92.

Galagan, James E, Calvo, Sarah E, Cuomo, Christina, Ma, Li-Jun, Wortman, Jennifer R, Batzoglou, Serafim, Lee, Su-In, Baştürkmen, Meray, Spevak, Christina C, Clutterbuck, John, et al.

"Sequencing of Aspergillus nidulans and comparative analysis with A. fumigatus and A. oryzae." Nature 438.7071 (2005): 1105-1115.

Galazka, Jonathan M, Tian, Chaoguang, Beeson, William T, Martinez, Bruno, Glass, N Louise, and Cate, Jamie HD. "Cellodextrin transport in yeast for improved biofuel production."

Science 330.6000 (2010): 84-86.

Gallmetzer, Martin and Burgstaller, Wolfgang.

"Efflux of organic acids in Penicillium simplicissimum is an energy-spilling process, adjusting the catabolic carbon flow to the nutrient supply and the activity of catabolic pathways."

Microbiology 148.4 (2002): 1143-1149.

Gietz, R Daniel and Woods, Robin A.

"Transformation of yeast by lithium acetate/single-stranded carrier DNA/polyethylene glycol method."

Methods in enzymology 350: 87-96.

Goldberg, Israel, Rokem, J Stefan, and Pines, Ophry.

"Organic acids: old metabolites, new themes."

Journal of Chemical Technology and Biotechnology 81.10 (2006): 1601-1611.

Goosen, Theo, Bloemheuvel, Gerda, Gysler, Christoph, Bie, Dick A, Broek, Henk WJ, and Swart, Klaas.

“Transformation of Aspergillus niger using the homologous orotidine-5'-phosphate-decarboxylase gene."

Current genetics 11.6 (1987): 499-503.

Goosen, Theo, Engelenburg, Frank, Debets, Fons, Swart, Klaas, Bos, Kees, and Broek, Henk.

"Tryptophan auxotrophic mutants in Aspergillus niger: inactivation of the trpC gene by cotransformation mutagenesis."

Molecular and General Genetics MGG 219.1 (1989): 282-288.

Granger, L-M, Perlot, P, Goma, G, and Pareilleux, A.

"Efficiency of fatty acid synthesis by oleaginous yeasts: prediction of yield and fatty acid cell content from consumed C/N ratio by a simple method."

Biotechnology and bioengineering 42.10 (1993): 1151-1156

Gruben, Birgit S, Zhou, Miaomiao, Wiebenga, Ad, Ballering, Joost, Overkamp, Karin M, Punt, Peter J, and De Vries, Ronald P. "Aspergillus niger RhaR, a regulator involved in L-rhamnose release and catabolism."

Applied microbiology and biotechnology 98.12 (2014): 5531.

Gründlinger, Mario, Yasmin, Sabiha, Lechner, Beatrix Elisabeth, Geley, Stephan, Schrettl, Markus, Hynes, Michael, and Haas, Hubertus.

"Fungal siderophore biosynthesis is partially localized in peroxisomes." 
Molecular microbiology 88.5 (2013): 862-875.

$\mathrm{Gu}$, Shuai, Xu, Qing, Huang, He, and Li, Shuang.

"Alternative respiration and fumaric acid production of Rhizopus oryzae."

Applied microbiology and biotechnology 98.11 (2014): 5145.

Guan, Lan and Kaback, H Ronald.

"Lessons from lactose permease."

Annu. Rev. Biophys. Biomol. Struct. 35: 67-91.

Guerinot, Mary Lou, Meidl, Erik J, and Plessner, Ora.

"Citrate as a siderophore in Bradyrhizobium japonicum."

Journal of bacteriology 172.6 (1990): 3298-3303.

Guerinot, Mary Lou and Yi, Ying.

"Iron: nutritious, noxious, and not readily available."

Plant Physiology 104.3 (1994): 815.

Gunn, Frank J, Tate, Christopher G, and Henderson, Peter JF.

"Identification of a novel sugar- $\mathrm{H}^{+}$symport protein, FucP, for transport of L-fucose into Escherichia coli."

Molecular microbiology 12.5 (1994): 799-809.

Haas, $\mathrm{H}$.

"Molecular genetics of fungal siderophore biosynthesis and uptake: the role of siderophores in iron uptake and storage."

Applied microbiology and biotechnology 62.4 (2003): 316-330.

Haas, Hubertus.

"Iron-a key nexus in the virulence of Aspergillus fumigatus."

Frontiers in microbiology 3.

"Fungal siderophore metabolism with a focus on Aspergillus fumigatus."

Natural product reports 31.10 (2014): 1266-1276.

Hamacher, Tanja, Becker, Jessica, Gárdonyi, Márk, Hahn-Hägerdal, Bärbel, and Boles, Eckhard

"Characterization of the xylose-transporting properties of yeast hexose transporters and their influence on xylose utilization." Microbiology 148.9 (2002): 2783-2788.

Han, Ying, Joosten, Henk-Jan, Niu, Weiling, Zhao, Zhiming, Mariano, Patrick S, McCalman, Melisa, Van Kan, Jan, Schaap, Peter J, and Dunaway-Mariano, Debra.

"Oxaloacetate hydrolase, the C-C bond lyase of oxalate secreting fungi."

Journal of Biological Chemistry 282.13 (2007): 9581-9590.

Hasper, Alinda A, Trindade, Luisa M, van der Veen, Douwe, van Ooyen, Albert JJ, and de Graaff, Leo H.

"Functional analysis of the transcriptional activator XlnR from Aspergillus niger."

Microbiology 150.5 (2004): 1367-1375.

Hector, Ronald E, Mertens, Jeffrey A, Bowman, Michael J, Nichols, Nancy N, Cotta, Michael A, and Hughes, Stephen R.

"Saccharomyces cerevisiae engineered for xylose metabolism requires gluconeogenesis and the oxidative branch of the pentose phosphate pathway for aerobic xylose assimilation."

Yeast 28.9 (2011): 645-660.

Hilditch, Satu, Berghäll, Suvi, Kalkkinen, Nisse, Penttilä, Merja, and Richard, Peter.

"The missing link in the fungal D-galacturonate pathway Identification of the L-threo-3-deoxy-hexulosonate aldolase."

Journal of Biological Chemistry 282.36 (2007): 26195-26201.

Hong, Seung-Beom, Lee, Mina, Kim, Dae-Ho, Varga, Janos, Frisvad, Jens C, Perrone, Giancarlo, Gomi, Katsuya, Yamada, Osamu, Machida, Masayuki, Houbraken, Jos, et al.

"Aspergillus luchuensis, an industrially important black Aspergillus in East Asia."

PLoS One 8.5 (2013): e63769.

Hortschansky, Peter, Eisendle, Martin, Al-Abdallah, Qusai, Schmidt, André D, Bergmann, Sebastian, Thön, Marcel, Kniemeyer, Olaf, Abt, Beate, Seeber, Birgit, Werner, Ernst R, et al.

"Interaction of HapX with the CCAAT-binding complex,Äîa novel mechanism of gene regulation by iron."

The EMBO journal 26.13 (2007): 3157-3168.

Huang, Zhen-Bang, Chen, Xiu-Zhen, Qin, Li-Na, Wu, Hong-Qing, Su, Xiao-Yun, and Dong, Zhi-Yang.

"A novel major facilitator transporter TrSTR1 is essential for pentose utilization and involved in xylanase induction in Trichoderma reesei."

Biochemical and biophysical research communications 460.3 (2015): 663-669.

Ihaka, Ross and Gentleman, Robert.

"R: a language for data analysis and graphics."

Journal of computational and graphical statistics 5.3 (1996): 299-314.

Jack, Donald L, Yang, Nelson M, and H Saier, Milton.

"The drug/metabolite transporter superfamily."

The FEBS Journal 268.13 (2001): 3620-3639.

Jang, Yu-Sin, Kim, Byoungjin, Shin, Jae Ho, Choi, Yong Jun, Choi, Sol, Song, Chan Woo, Lee, Joungmin, Park, Hye Gwon, and Lee, Sang Yup.

"Bio-based production of C2-C6 platform chemicals." 
Biotechnology and bioengineering 109.10 (2012): 2437-2459.

Janicka-Russak, Małgorzata, Kabała, Katarzyna, and Burzyński, Marek.

"Different effect of cadmium and copper on $\mathrm{H}^{+}$-ATPase activity in plasma membrane vesicles from Cucumis sativu s roots."

Journal of experimental botany 63.11 (2012): 4133-4142.

Jernejc, Katarina and Legiša, Matic.

"The influence of metal ions on malic enzyme activity and lipid synthesis in Aspergillus niger."

FEMS microbiology letters 217.2 (2002): 185-190.

Johnson, Mark A, Vidoni, Sara, Durigon, Romina, Pearce, Sarah F, Rorbach, Joanna, He, Jiuya, Brea-Calvo, Gloria, Minczuk, Michal, Reyes, Aurelio, Holt, Ian J, et al.

"Amino acid starvation has opposite effects on mitochondrial and cytosolic protein synthesis."

PloS one 9.4 (2014): e93597.

Jørgensen, Thomas R, Poulsen, Bjarne R, Ruijter, George JG, Visser, Jaap, Iversen, Jens JL, et al.

"Glucose uptake and growth of glucose-limited chemostat cultures of Aspergillus niger and a disruptant lacking MstA, a highaffinity glucose transporter."

Microbiology 153.6 (2007): 1963-1973.

Joseph-Horne, Tim, Hollomon, Derek W, and Wood, Paul M.

"Fungal respiration: a fusion of standard and alternative components."

Biochimica et Biophysica Acta (BBA)-Bioenergetics 1504.2 (2001): 179-195.

Joyce, Andrew R and Palsson, Bernhard Ø.

"The model organism as a system: integrating 'omics' data sets."

Nature Reviews Molecular Cell Biology 7.3 (2006): 198-210.

Kanehisa, Minoru and Goto, Susumu.

"KEGG: kyoto encyclopedia of genes and genomes."

Nucleic acids research 28.1 (2000): 27-30.

Kanehisa, Minoru, Sato, Yoko, Kawashima, Masayuki, Furumichi, Miho, and Tanabe, Mao.

"KEGG as a reference resource for gene and protein annotation."

Nucleic acids research 44.D1 (2016): D457-D462.

Karaffa, Levente and Kubicek, Christian P.

"Aspergillus niger citric acid accumulation: do we understand this well working black box?"

Applied microbiology and biotechnology 61.3 (2003): 189-196.

Kasahara, Toshiko, Shimogawara, Kosuke, and Kasahara, Michihiro.

"Crucial effects of amino acid side chain length in transmembrane segment 5 on substrate affinity in yeast glucose transporter Hxt7." Biochemistry 50.40 (2011): 8674-8681.

Kenealy, William, Zaady, Eli, du Preez, James C, Stieglitz, Barry, and Goldberg, Israel.

"Biochemical aspects of fumaric acid accumulation by Rhizopus arrhizus." Applied and environmental microbiology 52.1 (1986): 128-133.

Kim, Hyun, Melén, Karin, Österberg, Marie, and von Heijne, Gunnar. "A global topology map of the Saccharomyces cerevisiae membrane proteome." Proceedings of the National Academy of Sciences 103.30 (2006): 11142-11147.

Klipp, Edda, Nordlander, Bodil, Krüger, Roland, Gennemark, Peter, and Hohmann, Stefan. "Integrative model of the response of yeast to osmotic shock." Nature biotechnology 23.8 (2005): 975-982.

Knoshaug, Eric P, Vidgren, Virve, Magalhães, Frederico, Jarvis, Eric E, Franden, Mary Ann, Zhang, Min, and Singh, Arjun. "Novel transporters from Kluyveromyces marxianus and Pichia guilliermondii expressed in Saccharomyces cerevisiae enable growth on l-arabinose and d-xylose." Yeast 32.10 (2015): 615-628.

Koike, Hideaki, Aerts, Andrea, LaButti, Kurt, Grigoriev, Igor V, and Baker, Scott E. "Comparative genomics analysis of Trichoderma reesei strains." Industrial Biotechnology 9.6 (2013): 352-367.

Koivistoinen, Outi M, Arvas, Mikko, Headman, Jennifer R, Andberg, Martina, Penttilä, Merja, Jeffries, Thomas W, and Richard, Peter. "Characterisation of the gene cluster for l-rhamnose catabolism in the yeast Scheffersomyces (Pichia) stipitis." Gene 492.1 (2012): 177-185.

Kopylova, Evguenia, Noé, Laurent, and Touzet, Hélène.

"SortMeRNA: fast and accurate filtering of ribosomal RNAs in metatranscriptomic data." Bioinformatics 28.24 (2012): 3211-3217.

Kötter, Peter and Ciriacy, Michael.

"Xylose fermentation by Saccharomyces cerevisiae."

Applied microbiology and biotechnology 38.6 (1993): 776-783.

Krogh, Anders, Larsson, BjoÈrn, Von Heijne, Gunnar, and Sonnhammer, Erik LL.

"Predicting transmembrane protein topology with a hidden Markov model: application to complete genomes."

Journal of molecular biology 305.3 (2001): 567-580.

Kruis, Aleksander J, Levisson, Mark, Mars, Astrid E, van der Ploeg, Max, Daza, Fernando Garcés, Ellena, Valeria, Kengen, Servé WM, 
van der Oost, John, and Weusthuis, Ruud A.

"Ethyl acetate production by the elusive alcohol acetyltransferase from yeast."

Metabolic Engineering 41: 92-101.

Kubicek, CP and Röhr, M.

"Influence of manganese on enzyme synthesis and citric acid accumulation in Aspergillus niger."

Applied Microbiology and Biotechnology 4.3 (1977): 167-175.

"The role of the tricarboxylic acid cycle in citric acid accumulation by Aspergillus niger."

Applied Microbiology and Biotechnology 5.4 (1978): 263-271.

"Aconitase and citric acid fermentation by Aspergillus niger."

Applied and environmental microbiology 50.5 (1985): 1336-1338.

Kubicek, CP, Schreferl-Kunar, G, Wöhrer, W, and Röhr, M.

"Evidence for a cytoplasmic pathway of oxalate biosynthesis in Aspergillus niger."

Applied and Environmental Microbiology 54.3 (1988): 633-637.

Kusters-van Someren, Margo A, Harmsen, Jan AM, Kester, Harry CM, and Visser, Jaap.

"Structure of the Aspergillus niger pelA gene and its expression in Aspergillus niger and Aspergillus nidulans."

Current genetics 20.4 (1991): 293-299.

La Nauze, Julia M.

"Aconitase and isocitric dehydrogenases of Aspergillus niger in relation to citric acid production."

Microbiology 44.1 (1966): 73-81.

Lahaye, Marc and Robic, Audrey.

"Structure and functional properties of ulvan, a polysaccharide from green seaweeds."

Biomacromolecules 8.6 (2007): 1765-1774.

Langmead, Ben and Salzberg, Steven L.

"Fast gapped-read alignment with Bowtie 2."

Nature methods 9.4 (2012): 357-359.

Leandro, Maria José, Fonseca, Cesar, and Gonçalves, Paula.

"Hexose and pentose transport in ascomycetous yeasts: an overview."

FEMS Yeast Research 9.4 (2009): 511-525.

Leandro, Maria José, Gonçalves, Paula, and Spencer-Martins, Isabel.

"Two glucose/xylose transporter genes from the yeast Candida intermedia: first molecular characterization of a yeast xylose- $\mathrm{H}^{+}$ symporter."

Biochemical Journal 395.3 (2006): 543-549.

Leandro, Maria José, Sychrová, Hana, Prista, Catarina, and Loureiro-Dias, Maria C.

"ZrFsy1, a high-affinity fructose/ $\mathrm{H}^{+}$symporter from fructophilic yeast Zygosaccharomyces rouxii."

PLoS One 8.7 (2013): e68165.

Leangon, S, Maddox, IS, and Brooks, JD.

"A proposed biochemical mechanism for citric acid accumulation by Aspergillus niger Yang No. 2 growing in solid state fermentation."

World Journal of Microbiology and Biotechnology 16.3 (2000): 271-275.

Lee, SangYup, Mattanovich, Diethard, and Villaverde, Antonio.

"Systems metabolic engineering, industrial biotechnology and microbial cell factories."

Microbial cell factories 11.1 (2012): 156.

Legiša, Matic and Mattey, Michael.

"Changes in primary metabolism leading to citric acid overflow in Aspergillus niger."

Biotechnology letters 29.2 (2007): 181-190.

Lenouvel, Francois, van de Vondervoort, Peter J, and Visser, Jaap.

"Disruption of the Aspergillus niger argB gene: a tool for transformation."

Current genetics 41.6 (2002): 425-432.

Li, Ning, Zhang, Bo, Wang, Zhiwen, Tang, Ya-Jie, Chen, Tao, and Zhao, Xueming.

"Engineering Escherichia coli for fumaric acid production from glycerol."

Bioresource technology 174: 81-87.

Lin, MS and Wang, Hsi-Hua.

"Anaerobic growth and oxygen toxicity of Rhizopus cultures isolated from starters made by solid state fermentation."

Zhonghua Minguo wei sheng wu ji mian yi xue za zhi=Chinese journal of microbiology and immunology 24.2 (1991): 229-239.

Lubertozzi, David and Keasling, Jay D.

"Developing Aspergillus as a host for heterologous expression."

Biotechnology advances 27.1 (2009): 53-75.

Ma, Li-Jun, Ibrahim, Ashraf S, Skory, Christopher, Grabherr, Manfred G, Burger, Gertraud, Butler, Margi, Elias, Marek, Idnurm,

Alexander, Lang, B Franz, Sone, Teruo, et al.

"Genomic analysis of the basal lineage fungus Rhizopus oryzae reveals a whole-genome duplication."

PLoS Genet 5.7 (2009): e1000549. 
Mach-Aigner, Astrid R, Omony, Jimmy, Jovanovic, Birgit, van Boxtel, Anton JB, and de Graaff, Leo H.

"D-Xylose concentration-dependent hydrolase expression profiles and the function of CreA and XlnR in Aspergillus niger."

Applied and environmental microbiology 78.9 (2012): 3145-3155.

Madej, M Gregor and Kaback, H Ronald.

"Evolutionary mix-and-match with MFS transporters II."

Proceedings of the National Academy of Sciences 110.50 (2013): E4831-E4838.

Magnuson, Jon K and Lasure, Linda L.

"Organic acid production by filamentous fungi."

Advances in fungal biotechnology for industry, agriculture, and medicine. Springer, 2004.

307-340.

Maier, Andreas, Völker, Bernhard, Boles, Eckhard, and Fuhrmann, Günter Fred.

"Characterisation of glucose transport in Saccharomyces cerevisiae with plasma membrane vesicles (countertransport) and intact cells (initial uptake) with single Hxt1, Hxt2, Hxt3, Hxt4, Hxt6, Hxt7 or Gal2 transporters.”

FEMS yeast research 2.4 (2002): 539-550.

Mandels, Mary.

"Applications of cellulases."

1985.

Martens-Uzunova, Elena S and Schaap, Peter J.

"An evolutionary conserved D-galacturonic acid metabolic pathway operates across filamentous fungi capable of pectin degradation."

Fungal Genetics and Biology 45.11 (2008): 1449-1457.

"Assessment of the pectin degrading enzyme network of Aspergillus niger by functional genomics."

Fungal Genetics and Biology 46.1 (2009): S170-S179.

Martin, Marcel.

"Cutadapt removes adapter sequences from high-throughput sequencing reads."

EMBnet. journal 17.1 (2011): pp-10.

Martinez, Diego, Berka, Randy M, Henrissat, Bernard, Saloheimo, Markku, Arvas, Mikko, Baker, Scott E, Chapman, Jarod, Chertkov, Olga, Coutinho, Pedro M, Cullen, Dan, et al.

"Genome sequencing and analysis of the biomass-degrading fungus Trichoderma reesei (syn. Hypocrea jecorina)."

Nature biotechnology 26.5 (2008): 553-560.

Meijer, Susan, Nielsen, Michael Lynge, Olsson, Lisbeth, and Nielsen, Jens.

"Gene deletion of cytosolic ATP: citrate lyase leads to altered organic acid production in Aspergillus niger."

Journal of industrial microbiology \& biotechnology 36.10 (2009): 1275-1280.

Meixner-Monori, B, Kubicek, Ch P, Harrer, W, Schreferl, G, and Rohr, M.

"NADP-specific isocitrate dehydrogenase from the citric acid-accumulating fungus Aspergillus niger."

Biochemical Journal 236.2 (1986): 549-557.

Meixner-Monori, BIBIANA, Kubicek, Ch P, Habison, AYSEN, Kubicek-Pranz, EM, and Röhr, M.

"Presence and regulation of the alpha-ketoglutarate dehydrogenase multienzyme complex in the filamentous fungus Aspergillus niger."

Journal of bacteriology 161.1 (1985): 265-271.

Mello-de Sousa, Thiago M, Rassinger, Alice, Pucher, Marion E, dos Santos Castro, Lilian, Persinoti, Gabriela F, Silva-Rocha, Rafael, Poças-Fonseca, Marcio J, Mach, Robert L, Silva, Roberto Nascimento, and Mach-Aigner, Astrid R.

"The impact of chromatin remodelling on cellulase expression in Trichoderma reesei."

BMC genomics 16.1 (2015): 588 .

Mesojednik, Suzana and Legiša, Matic.

"Posttranslational modification of 6-phosphofructo-1-kinase in Aspergillus niger."

Applied and environmental microbiology 71.3 (2005): 1425-1432.

Meussen, Bas J, de Graaff, Leo H, Sanders, Johan PM, and Weusthuis, Ruud A.

"Metabolic engineering of Rhizopus oryzae for the production of platform chemicals."

Applied microbiology and biotechnology 94.4 (2012): 875-886.

Meyer, Vera, Arentshorst, Mark, El-Ghezal, Aymen, Drews, Ann-Christin, Kooistra, Rolf, van den Hondel, Cees AMJJ, and Ram, Arthur FJ.

"Highly efficient gene targeting in the Aspergillus niger kusA mutant."

Journal of Biotechnology 128.4 (2007): 770-775.

Millenaar, Frank F, Gonzalez-Meler, Miquel A, Siedow, James N, Wagner, Anneke M, and Lambers, Hans.

"Role of sugars and organic acids in regulating the concentration and activity of the alternative oxidase in Poa annua roots." Journal of Experimental Botany 53.371 (2002): 1081-1088.

Minagawa, Nobuko, Sakajo, Shigeru, Komiyama, Tadazumi, and Yoshimoto, Akio.

"Essential role of ferrous iron in cyanide-resistant respiration in Hansenula anomala."

FEBS letters 267.1 (1990): 114-116.

Mishra, Nitish K, Chang, Junil, and Zhao, Patrick X.

"Prediction of membrane transport proteins and their substrate specificities using primary sequence information." 
PLoS One 9.6 (2014): e100278.

Mlakar, Tina and Legiša, Matic.

"Citrate inhibition-resistant form of 6-phosphofructo-1-kinase from Aspergillus niger."

Applied and environmental microbiology 72.7 (2006): 4515-4521.

Mohnen, Debra.

"Pectin structure and biosynthesis."

Current opinion in plant biology 11.3 (2008): 266-277.

Neilands, J Bo.

"Microbial iron compounds."

Annual review of biochemistry 50.1 (1981): 715-731.

Neilands, JB.

"Siderophores: structure and function of microbial iron transport compounds."

Journal of Biological Chemistry 270.45 (1995): 26723-26726.

Netik, Angela, Torres, Nestor V, Riol, Jose-Maria, and Kubicek, Christian P.

"Uptake and export of citric acid by Aspergillus niger is reciprocally regulated by manganese ions."

Biochimica et Biophysica Acta (BBA)-Biomembranes 1326.2 (1997): 287-294.

Neves, Luiz Carlos Martins and Vitolo, Michele.

"Use of glucose oxidase in a membrane reactor for gluconic acid production." Applied Biochemistry and Biotecnology : 161-170.

Nierman, William C, Pain, Arnab, Anderson, Michael J, Wortman, Jennifer R, Kim, H Stanley, Arroyo, Javier, Berriman, Matthew, Abe, Keietsu, Archer, David B, Bermejo, Clara, et al.

"Genomic sequence of the pathogenic and allergenic filamentous fungus Aspergillus fumigatus." Nature 438.7071 (2005): 1151-1156.

Nijland, Jeroen G, Shin, Hyun Yong, de Jong, René M, De Waal, Paul P, Klaassen, Paul, and Driessen, Arnold JM.

"Engineering of an endogenous hexose transporter into a specific D-xylose transporter facilitates glucose-xylose co-consumption in Saccharomyces cerevisiae."

Biotechnology for biofuels 7.1 (2014): 168

Niu, Jing, Homan, Tim G, Arentshorst, Mark, de Vries, Ronald P, Visser, Jaap, and Ram, Arthur FJ.

"The interaction of induction and repression mechanisms in the regulation of galacturonic acid-induced genes in Aspergillus niger."

Fungal Genetics and Biology 82: 32-42.

Nordberg, Henrik, Cantor, Michael, Dusheyko, Serge, Hua, Susan, Poliakov, Alexander, Shabalov, Igor, Smirnova, Tatyana, Grigoriev, Igor $\mathrm{V}$, and Dubchak, Inna.

"The genome portal of the Department of Energy Joint Genome Institute: 2014 updates."

Nucleic acids research 42.D1 (2013): D26-D31.

Odoni, Dorett I, Tamayo-Ramos, Juan A, Sloothaak, Jasper, van Heck, Ruben GA, dos Santos, Vitor AP Martins, de Graaff, Leo H, Suarez-Diez, Maria, and Schaap, Peter J.

"Comparative proteomics of Rhizopus delemar ATCC 20344 unravels the role of amino acid catabolism in fumarate accumulation." PeerJ 5: e3133.

Odoni, Dorett I, van Gaal, Merlijn P, Schonewille, Tom, Tamayo-Ramos, Juan A, dos Santos, Vitor AP Martins, Suarez-Diez, Maria, and Schaap, Peter J.

"Aspergillus niger secretes citrate to increase iron bioavailability."

Frontiers in Microbiology 8.

Orthofer, R, Kubicek, CP, and Röhr, M.

"Lipid levels and manganese deficiency in citric acid producing strains of Aspergillus niger."

FEMS Microbiology Letters 5.6 (1979): 403-406.

Osmani, Stephen A and Scrutton, Michael C.

"The sub-cellular localisation and regulatory properties of pyruvate carboxylase from Rhizopus arrhizus."

European journal of biochemistry 147.1 (1985): 119-128.

Ouyang, Haomiao, Luo, Yuanming, Zhang, Lei, Li, Yanjie, and Jin, Cheng.

"Proteome analysis of Aspergillus fumigatus total membrane proteins identifies proteins associated with the glycoconjugates and cell wall biosynthesis using 2D LC-MS/MS."

Molecular biotechnology 44.3 (2010): 177-189.

Overman, Sue Ann and Romano, Antonio H.

"Pyruvate carboxylase of Rhizopus nigricans and its role in fumaric acid production."

Biochemical and biophysical research communications 37.3 (1969): 457-463.

Owen, Oliver E, Kalhan, Satish C, and Hanson, Richard W.

"The key role of anaplerosis and cataplerosis for citric acid cycle function."

Journal of Biological Chemistry 277.34 (2002): 30409-30412.

Ozcan, Sabire, Dover, Jim, Rosenwald, Anne G, Wölfl, S, and Johnston, Mark.

"Two glucose transporters in Saccharomyces cerevisiae are glucose sensors that generate a signal for induction of gene expression." Proceedings of the National Academy of Sciences 93.22 (1996): 12428-12432.

Özcan, Sabire and Johnston, Mark. 
"Function and regulation of yeast hexose transporters."

Microbiology and Molecular Biology Reviews 63.3 (1999): 554-569.

Pao, Stephanie S, Paulsen, Ian T, and Saier, Milton H.

"Major facilitator superfamily."

Microbiology and molecular biology reviews 62.1 (1998): 1-34.

Pardo, Ester and Orejas, Margarita.

"The Aspergillus nidulans Zn (II) 2 Cys 6 transcription factor AN5673/RhaR mediates L-rhamnose utilization and the production of $\alpha$-L-rhamnosidases."

Microbial cell factories 13.1 (2014): 161.

Patel, Vibhuti J, Thalassinos, Konstantinos, Slade, Susan E, Connolly, Joanne B, Crombie, Andrew, Murrell, J Colin, and Scrivens, James H.

"A comparison of labeling and label-free mass spectrometry-based proteomics approaches."

Journal of proteome research 8.7 (2009): 3752-3759.

Patil, Kiran Raosaheb, Åkesson, Mats, and Nielsen, Jens.

"Use of genome-scale microbial models for metabolic engineering."

Current opinion in biotechnology 15.1 (2004): 64-69.

Paulsen, Ian T, Chauvaux, Sylvie, Choi, Peter, and Saier, Milton H.

"Characterization of glucose-specific catabolite repression-resistant mutants of Bacillus subtilis: identification of a novel hexose: $\mathrm{H}^{+}$symporter."

Journal of bacteriology 180.3 (1998): 498-504.

Pazur, John $\mathrm{H}$ and Kleppe, Kjell.

"The oxidation of glucose and related compounds by glucose oxidase from Aspergillus niger."

Biochemistry 3.4 (1964): 578-583.

Peij, Noel NME, Brinkmann, Joep, Vršanská, Mária, Visser, Jaap, and Graaff, Leo H.

" $\beta$-Xylosidase Activity, Encoded by $x \operatorname{lnD}$, is Essential for Complete Hydrolysis of Xylan by Aspergillus Niger but not for Induction of the Xylanolytic Enzyme Spectrum."

European Journal of Biochemistry 245.1 (1997): 164-173.

Peleg, Yoav, Battat, Emil, Scrutton, Michael C, and Goldberg, Israel.

"Isoenzyme pattern and subcellular localisation of enzymes involved in fumaric acid accumulation by Rhizopus oryzae."

Applied microbiology and biotechnology 32.3 (1989): 334-339.

Peng, Yu, Leung, Henry CM, Yiu, Siu-Ming, and Chin, Francis YL.

"IDBA-UD: a de novo assembler for single-cell and metagenomic sequencing data with highly uneven depth." Bioinformatics 28.11 (2012): 1420-1428.

Petersen, Thomas Nordahl, Brunak, Søren, von Heijne, Gunnar, and Nielsen, Henrik.

"SignalP 4.0: discriminating signal peptides from transmembrane regions."

Nature methods 8.10 (2011): 785-786

Pfitzner, A, Kubicek, CP, and Röhr, M.

"Presence and regulation of ATP: citrate lyase from the citric acid producing fungus Aspergillus niger." Archives of microbiology 147.1 (1987): 88-91.

Polidori, Emanuela, Ceccaroli, Paola, Saltarelli, Roberta, Guescini, Michele, Menotta, Michele, Agostini, Deborah, Palma, Francesco, and Stocchi, Vilberto.

"Hexose uptake in the plant symbiotic ascomycete Tuber borchii Vittadini: biochemical features and expression pattern of the transporter TBHXT1."

Fungal Genetics and Biology 44.3 (2007): 187-198.

Pontecorvo, Gr, Roper, JA, Chemmons, LM, MacDonald, KD, and Bufton, AWJ.

"The genetics of Aspergillus nidulans."

Advances in genetics 5: 141-238.

Psakis, Georgios, Saidijam, Massoud, Shibayama, Keigo, Polaczek, Julia, Bettaney, Kim E, Baldwin, Jocelyn M, Baldwin, Stephen A, Hope, Ryan, Essen, Lars-Oliver, Essenberg, Richard C, et al.

"The sodium-dependent d-glucose transport protein of Helicobacter pylori."

Molecular microbiology 71.2 (2009): 391-403.

Pullan, Steven T, Daly, Paul, Delmas, Stéphane, Ibbett, Roger, Kokolski, Matthew, Neiteler, Almar, van Munster, Jolanda M, Wilson, Raymond, Blythe, Martin J, Gaddipati, Sanyasi, et al.

"RNA-sequencing reveals the complexities of the transcriptional response to lignocellulosic biofuel substrates in Aspergillus niger." Fungal biology and biotechnology 1.1 (2014): 3.

Punt, Peter J, van Biezen, Nick, Conesa, Ana, Albers, Alwin, Mangnus, Jeroen, and van den Hondel, Cees.

"Filamentous fungi as cell factories for heterologous protein production."

Trends in biotechnology 20.5 (2002): 200-206.

Punta, Marco, Coggill, Penny C, Eberhardt, Ruth Y, Mistry, Jaina, Tate, John, Boursnell, Chris, Pang, Ningze, Forslund, Kristoffer, Ceric, Goran, Clements, Jody, et al.

"The Pfam protein families database."

Nucleic acids research 40.D1 (2011): D290-D301.

Quinlan, Aaron R and Hall, Ira M. 
"BEDTools: a flexible suite of utilities for comparing genomic features." Bioinformatics 26.6 (2010): 841-842.

Quistgaard, Esben M, Löw, Christian, Moberg, Per, Trésaugues, Lionel, and Nordlund, Pär. "Structural basis for substrate transport in the GLUT-homology family of monosaccharide transporters." Nature structural \& molecular biology 20.6 (2013): 766-768.

Rajala, Nina, Hensen, Fenna, Wessels, Hans JCT, Ives, Daniel, Gloerich, Jolein, and Spelbrink, Johannes N. "Whole cell formaldehyde cross-linking simplifies purification of mitochondrial nucleoids and associated proteins involved in mitochondrial gene expression." PLoS One 10.2 (2015): e0116726.

Ramos, Augusto SP, Chambergo, Felipe S, Bonaccorsi, Eric D, Ferreira, Ari JS, Cella, Nathalie, Gombert, Andreas K, Tonso, Aldo, and El-Dorry, Hamza.

"Oxygen-and glucose-dependent expression of Trhxt1, a putative glucose transporter gene of Trichoderma reesei." Biochemistry 45.26 (2006): 8184-8192.

Rappsilber, Juri, Ishihama, Yasushi, and Mann, Matthias.

"Stop and go extraction tips for matrix-assisted laser desorption/ionization, nanoelectrospray, and LC/MS sample pretreatment in proteomics." Analytical chemistry 75.3 (2003): 663-670.

Ratledge, Colin. "Look before you clone." FEMS microbiology letters 189.2 (2000): 317-318.

Rautengarten, Carsten, Ebert, Berit, Moreno, Ignacio, Temple, Henry, Herter, Thomas, Link, Bruce, Doñas-Cofré, Daniela, Moreno, Adrián, Saéz-Aguayo, Susana, Blanco, Francisca, et al.

"The Golgi localized bifunctional UDP-rhamnose/UDP-galactose transporter family of Arabidopsis." Proceedings of the National Academy of Sciences 111.31 (2014): 11563-11568.

Reifenberger, E, Freidel, K, and Ciriacy, M. "Identification of novel HXT genes in Saccharomyces cerevisiae reveals the impact of individual hexose transporters on qlycolytic flux." Molecular microbiology 16.1 (1995): 157-167.

Reifenberger, Elke, Boles, Eckhard, and Ciriacy, Michael.

"Kinetic characterization of individual hexose transporters of Saccharomyces cerevisiae and their relation to the triggering mechanisms of glucose repression." The FEBS Journal 245.2 (1997): 324-333.

Reznicek, Ondrej, Facey, Sandra J, Waal, PP, Teunissen, Aloys WRH, Bont, JAM, Nijland, Jeroen G, Driessen, Arnold JM, and Hauer, Bernhard.

"Improved xylose uptake in Saccharomyces cerevisiae due to directed evolution of galactose permease Gal2 for sugar coconsumption." Journal of applied microbiology 119.1 (2015): 99-111.

Rhodes, RA, Moyer, AJ, Smith, Mabel L, and Kelley, Sinah E. "Production of fumaric acid by Rhizopus arrhizus." Applied microbiology 7.2 (1959): 74.

Ries, Laure, Pullan, Steven T, Delmas, Stéphane, Malla, Sunir, Blythe, Martin J, and Archer, David B. "Genome-wide transcriptional response of Trichoderma reesei to lignocellulose using RNA sequencing and comparison with Aspergillus niger." BMC genomics 14.1 (2013): 541.

Robinson, Mark D, McCarthy, Davis J, and Smyth, Gordon K "edgeR: a Bioconductor package for differential expression analysis of digital gene expression data." Bioinformatics 26.1 (2010): 139-140.

Rogers, P David, Vermitsky, John-Paul, Edlind, Thomas D, and Hilliard, George M. "Proteomic analysis of experimentally induced azole resistance in Candida glabrata." Journal of Antimicrobial Chemotherapy 58.2 (2006): 434-438.

Romano, Antonio H, Bright, Marlene M, and Scott, William E. "Mechanism of fumaric acid accumulation in Rhizopus nigricans." Journal of Bacteriology 93.2 (1967): 600-604.

Ronning, Catherine M, Fedorova, Natalie D, Bowyer, Paul, Coulson, Richard, Goldman, Gustavo, Kim, H Stanley, Turner, Geoffrey, Wortman, Jennifer R, Yu, Jiujiang, Anderson, Michael J, et al. "Genomics of Aspergillus fumigatus." Revista iberoamericana de micología 22.4 (2005): 223-228.

Ruijter, George. "Life is not that simple." FEMS Microbiology Letters 189.2 (2000): 318-319.

Ruijter, George JG, Bax, Maarten, Patel, Hema, Flitter, Simon J, van de Vondervoort, Peter JI, de Vries, Ronald P, Visser, Jaap, et al. "Mannitol is required for stress tolerance in Aspergillus niger conidiospores." Eukaryotic Cell 2.4 (2003): 690-698. 
Ruijter, George JG, Panneman, Henk, Xu, Ding-Bang, and Visser, Jaap.

"Properties of Aspergillus niger citrate synthase and effects of citA overexpression on citric acid production."

FEMS microbiology letters 184.1 (2000): 35-40.

Ruijter, George JG, van de Vondervoort, Peter JI, and Visser, Jaap.

"Oxalic acid production by Aspergillus niger: an oxalate-non-producing mutant produces citric acid at pH 5 and in the presence of manganese."

Microbiology 145.9 (1999): 2569-2576

Ruijter, George JG, Vanhanen, Sipo A, Gielkens, Marco MC, van de Vondervoort, Peter JI, and Visser, Jaap.

"Isolation of Aspergillus niger creA mutants and effects of the mutations on expression of arabinases and L-arabinose catabolic enzymes."

Microbiology 143.9 (1997a): 2991-2998.

Ruijter, GJG, Panneman, H, and Visser, J.

"Overexpression of phosphofructokinase and pyruvate kinase in citric acid-producing Aspergillus niger."

Biochimica et Biophysica Acta (BBA)-General Subjects 1334.2 (1997b): 317-326.

Saier, Milton H.

"Families of transmembrane sugar transport proteins."

Molecular microbiology 35.4 (2000): 699-710.

Saloheimo, Anu, Rauta, Jenita, Stasyk, Oleh V, Sibirny, Andrei A, Penttilä, Merja, and Ruohonen, Laura.

"Xylose transport studies with xylose-utilizing Saccharomyces cerevisiae strains expressing heterologous and homologous permeases."

Applied microbiology and biotechnology 74.5 (2007): 1041-1052.

Sass, Ehud, Blachinsky, Eran, Karniely, Sharon, and Pines, Ophry.

"Mitochondrial and cytosolic isoforms of yeast fumarase are derivatives of a single translation product and have identical amino termini."

Journal of Biological Chemistry 276.49 (2001): 46111-46117.

Schmieder, Robert and Edwards, Robert.

"Quality control and preprocessing of metagenomic datasets."

Bioinformatics 27.6 (2011): 863-864.

Schomburg, Ida, Chang, Antje, Ebeling, Christian, Gremse, Marion, Heldt, Christian, Huhn, Gregor, and Schomburg, Dietmar.

"BRENDA, the enzyme database: updates and major new developments."

Nucleic acids research 32.suppl_1 (2004): D431-D433.

Schrader, Michael and Fahimi, H Dariush.

"Peroxisomes and oxidative stress."

Biochimica et Biophysica Acta (BBA)-Molecular Cell Research 1763.12 (2006): 1755-1766.

Schrettl, Markus, Kim, H Stanley, Eisendle, Martin, Kragl, Claudia, Nierman, William C, Heinekamp, Thorsten, Werner, Ernst R, Jacobsen, Ilse, Illmer, Paul, Yi, Hyojeong, et al.

"SreA-mediated iron regulation in Aspergillus fumigatus."

Molecular microbiology 70.1 (2008): 27-43.

Schuster, André, Bruno, Kenneth S, Collett, James R, Baker, Scott E, Seiboth, Bernhard, Kubicek, Christian P, and Schmoll, Monika "A versatile toolkit for high throughput functional genomics with Trichoderma reesei."

Biotechnology for biofuels 5.1 (2012): 1.

Schuster, E, Dunn-Coleman, N, Frisvad, JC, and Van Dijck, P.

"On the safety of Aspergillus niger-a review."

Applied microbiology and biotechnology 59.4 (2002): 426-435.

Show, Pau Loke, Oladele, Kehinde Opeyemi, Siew, Qi Yan, Aziz Zakry, Fitri Abdul, Lan, John Chi-Wei, and Ling, Tau Chuan.

"Overview of citric acid production from Aspergillus niger."

Frontiers in Life Science 8.3 (2015): 271-283.

Sievers, Fabian, Wilm, Andreas, Dineen, David, Gibson, Toby J, Karplus, Kevin, Li, Weizhong, Lopez, Rodrigo, McWilliam, Hamish, Remmert, Michael, Söding, Johannes, et al.

"Fast, scalable generation of high-quality protein multiple sequence alignments using Clustal Omega."

Molecular systems biology 7.1 (2011): 539.

Silva, Andre MN, Kong, XiaoLe, Parkin, Mark C, Cammack, Richard, and Hider, Robert C.

"Iron (III) citrate speciation in aqueous solution."

Dalton Transactions .40 (2009): 8616-8625.

Simossis, Victor A and Heringa, Jaap.

"PRALINE: a multiple sequence alignment toolbox that integrates homology-extended and secondary structure information."

Nucleic acids research 33.suppl_2 (2005): W289-W294.

Singh, Bhag and Gupta, Radhey S.

"Mitochondrial import of human and yeast fumarase in live mammalian cells: retrograde translocation of the yeast enzyme is mainly caused by its poor targeting sequence."

Biochemical and biophysical research communications 346.3 (2006): 911-918.

Sloothaak, J, Odoni, DI, De Graaff, LH, Dos Santos, VAP Martins, Schaap, PJ, and Tamayo-Ramos, JA.

"Aspergillus niger membrane-associated proteome analysis for the identification of glucose transporters." 
Biotechnology for biofuels 8.1 (2015): 150.

Sloothaak, Jasper, Odoni, Dorett I, dos Santos, Vitor AP Martins, Schaap, Peter J, and Tamayo-Ramos, Juan Antonio.

"Identification of a Novel L-rhamnose Uptake Transporter in the Filamentous Fungus Aspergillus niger."

PLoS Genetics 12.12 (2016a): e1006468.

Sloothaak, Jasper, Schilders, Mike, Schaap, Peter J, and de Graaff, Leo H.

"Overexpression of the Aspergillus niger GatA transporter leads to preferential use of D-galacturonic acid over D-xylose." AMB Express 4.1 (2014): 66.

Sloothaak, Jasper, Tamayo-Ramos, Juan Antonio, Odoni, Dorett I, Laothanachareon, Thanaporn, Derntl, Christian, Mach-Aigner, Astrid R, Dos Santos, Vitor AP Martins, and Schaap, Peter J.

"Identification and functional characterization of novel xylose transporters from the cell factories Aspergillus niger and Trichoderma reesei."

Biotechnology for biofuels 9.1 (2016b): 148 .

Song, Chan Woo, Kim, Dong In, Choi, Sol, Jang, Jae Won, and Lee, Sang Yup.

"Metabolic engineering of Escherichia coli for the production of fumaric acid."

Biotechnology and bioengineering 110.7 (2013): 2025-2034.

Song, Ping, Li, Shuang, Ding, Yueyue, Xu, Qing, and Huang, He.

"Expression and characterization of fumarase (FUMR) from Rhizopus oryzae."

Fungal biology 115.1 (2011): 49-53.

Steiger, Matthias G, Mach, Robert L, and Mach-Aigner, Astrid R.

"An accurate normalization strategy for RT-qPCR in Hypocrea jecorina (Trichoderma reesei)."

Journal of biotechnology 145.1 (2010): 30-37.

Steiger, Matthias G, Vitikainen, Marika, Uskonen, Pekka, Brunner, Kurt, Adam, Gerhard, Pakula, Tiina, Penttilä, Merja, Saloheimo, Markku, Mach, Robert L, and Mach-Aigner, Astrid R.

"Transformation system for Hypocrea jecorina (Trichoderma reesei) that favors homologous integration and employs reusable bidirectionally selectable markers."

Applied and environmental microbiology 77.1 (2011): 114-121.

Straathof, Adrie JJ and van Gulik, Walter M.

"Production of fumaric acid by fermentation."

Reprogramming microbial metabolic pathways. Springer, 2012.

225-240.

Strauss, Joseph, Horvath, Henny K, Abdallah, Basem M, Kindermann, Johanna, Mach, Robert L, and Kubicek, Christian P.

"The function of CreA, the carbon catabolite repressor of Aspergillus nidulans, is regulated at the transcriptional and posttranscriptional level."

Molecular microbiology 32.1 (1999): 169-178.

Stricker, Astrid R, Grosstessner-Hain, Karin, Würleitner, Elisabeth, and Mach, Robert L.

"Xyr1 (xylanase regulator 1) regulates both the hydrolytic enzyme system and D-xylose metabolism in Hypocrea jecorina." Eukaryotic cell 5.12 (2006): 2128-2137.

Subtil, Thorsten and Boles, Eckhard.

"Improving L-arabinose utilization of pentose fermenting Saccharomyces cerevisiae cells by heterologous expression of L-arabinose transporting sugar transporters."

Biotechnology for biofuels 4.1 (2011): 38.

Suga, Minoru and Hatakeyama, Toyomasa.

"High-efficiency electroporation by freezing intact yeast cells with addition of calcium."

Current genetics 43.3 (2003): 206-211.

Sun, Linfeng, Zeng, Xin, Yan, Chuangye, Sun, Xiuyun, Gong, Xinqi, Rao, Yu, and Yan, Nieng.

"Crystal structure of a bacterial homologue of glucose transporters GLUT1-4."

Nature 490.7420 (2012): 361-366.

Suzuki, Tamio, Sato, M, Yoshida, T, and Tuboi, Syozo.

"Rat liver mitochondrial and cytosolic fumarases with identical amino acid sequences are encoded from a single gene."

Journal of Biological Chemistry 264.5 (1989): 2581-2586.

Szczodrak, J and Ilczuk, Z.

"Effect of iron on the activity of aconitate hydratase and synthesis of citric acid by Aspergillus niger."

Zentralblatt für Mikrobiologie 140.7 (1985): 567-574.

Szopinska, Aleksandra, Degand, Hervé, Hochstenbach, Jean-François, Nader, Joseph, and Morsomme, Pierre.

"Rapid response of the yeast plasma membrane proteome to salt stress."

Molecular \& Cellular Proteomics : mcp-M111.

Tamayo-Ramos, Juan A, Flipphi, Michel, Pardo, Ester, Manzanares, Paloma, and Orejas, Margarita.

"L-Rhamnose induction of Aspergillus nidulans $\alpha$-L-rhamnosidase genes is glucose repressed via a CreA-independent mechanism acting at the level of inducer uptake." Microbial cell factories 11.1 (2012): 26

Tamayo-Ramos, Juan Antonio and Orejas, Margarita.

"Enhanced glycosyl hydrolase production in Aspergillus nidulans using transcription factor engineering approaches."

Biotechnology for biofuels 7.1 (2014): 103. 
Tantirungkij, Manee, Nakashima, Noriyuki, Seki, Tatsuji, and Yoshida, Toshiomi.

"Construction of xylose-assimilating Saccharomyces cerevisiae."

Journal of Fermentation and Bioengineering 75.2 (1993): 83-88.

Tate, Christopher G, Muiry, JA, and Henderson, PJ.

"Mapping, cloning, expression, and sequencing of the rhaT gene, which encodes a novel L-rhamnose- $\mathrm{H}^{+}$transport protein in Salmonella typhimurium and Escherichia coli."

Journal of Biological Chemistry 267.10 (1992): 6923-6932.

Tepper, Naama and Shlomi, Tomer.

"Predicting metabolic engineering knockout strategies for chemical production: accounting for competing pathways."

Bioinformatics 26.4 (2009): 536-543.

Thomas, KC and Dawson, PSS.

"Relationship between iron-limited growth and energy limitation during phased cultivation of Candida utilis."

Canadian journal of microbiology 24.4 (1978): 440-447.

Torres, NV, Riol-Cimas, JM, Wolschek, M, and Kubicek, CP.

"Glucose transport by Aspergillus niger: the low-affinity carrier is only formed during growth on high glucose concentrations." Applied microbiology and biotechnology 44.6 (1996): 790-794.

Tsekova, K, Todorova, D, and Ganeva, S.

"Removal of heavy metals from industrial wastewater by free and immobilized cells of Aspergillus niger."

International Biodeterioration \& Biodegradation 64.6 (2010): 447-451.

van den Brink, Joost and de Vries, Ronald P.

"Fungal enzyme sets for plant polysaccharide degradation."

Applied microbiology and biotechnology 91.6 (2011): 1477-1492.

van der Straat, Laura and de Graaff, Leo $\mathrm{H}$.

"Pathway transfer in fungi: Transporters are the key to success."

Bioengineered 5.5 (2014): 335-339.

van der Straat, Laura, Vernooij, Marloes, Lammers, Marieke, van den Berg, Willy, Schonewille, Tom, Cordewener, Jan, van der Meer, Ingrid, Koops, Andries, and de Graaff, Leo H.

"Expression of the Aspergillus terreus itaconic acid biosynthesis cluster in Aspergillus niger."

Microbial cell factories 13.1 (2014): 11.

van der Veen, Douwe, Oliveira, José Miguel, Van den Berg, Willy AM, and de Graaff, Leo H.

"Analysis of variance components reveals the contribution of sample processing to transcript variation."

Applied and environmental microbiology 75.8 (2009): 2414-2422.

Van Leeuwen, MR, Krijgsheld, P, Wyatt, TT, Golovina, EA, Menke, H, Dekker, A, Stark, J, Stam, H, Bleichrodt, R, Wösten, HAB, et al. "The effect of natamycin on the transcriptome of conidia of Aspergillus niger."

Studies in mycology 74: 71-85.

van Peij, Noël NME, Gielkens, Marco MC, de Vries, Ronald P, Visser, Jaap, and de Graaff, Leo H.

"The transcriptional activator XlnR regulates both xylanolytic and endoglucanase gene expression in Aspergillus niger."

Applied and Environmental Microbiology 64.10 (1998): 3615-3619.

Van Peij, Noël NME, Visser, Jaap, and De Graaff, Leo H.

"Isolation and analysis of xlnR, encoding a transcriptional activator co-ordinating xylanolytic expression in Aspergillus niger."

Molecular microbiology 27.1 (1998): 131-142.

Van Regenmortel, Marc HV.

"Reductionism and complexity in molecular biology."

EMBO reports 5.11 (2004): 1016-1020.

Vankuyk PA, Jasper A, DIDERICH, MacCABE, Andrew P, HERERRO, Oscar, RUIJTER, George JG, VISSER, Jaap, et al. "Aspergillus niger mstA encodes a high-affinity sugar/ $\mathrm{H}^{+}$symporter which is regulated in response to extracellular $\mathrm{pH} . "$ Biochemical Journal 379.2 (2004): 375-383.

Vanlerberghe, Greg C.

"Alternative oxidase: a mitochondrial respiratory pathway to maintain metabolic and signaling homeostasis during abiotic and biotic stress in plants."

International Journal of Molecular Sciences 14.4 (2013): 6805-6847.

Vardy, Eyal, Arkin, Isaiah T, Gottschalk, Kay E, Kaback, H Ronald, and Schuldiner, Shimon.

"Structural conservation in the major facilitator superfamily as revealed by comparative modeling."

Protein Science 13.7 (2004): 1832-1840.

Verduyn, Cornelis, Postma, Erik, Scheffers, W Alexander, and Van Dijken, Johannes P.

"Effect of benzoic acid on metabolic fluxes in yeasts: a continuous-culture study on the regulation of respiration and alcoholic fermentation." Yeast 8.7 (1992): 501-517.

Vishniac, Wolf and Santer, Melvin. "The thiobacilli." Bacteriological reviews 21.3 (1957): 195.

Vizcaíno, Juan Antonio, Csordas, Attila, Del-Toro, Noemi, Dianes, José A, Griss, Johannes, Lavidas, Ilias, Mayer, Gerhard, PerezRiverol, Yasset, Reisinger, Florian, Ternent, Tobias, et al. 
"2016 update of the PRIDE database and its related tools." Nucleic acids research 44.D1 (2015): D447-D456.

Vrabl, Pamela, Fuchs, Viktoria, Pichler, Barbara, Schinagl, Christoph W, and Burgstaller, Wolfgang. "Organic acid excretion in Penicillium ochrochloron increases with ambient pH." Frontiers in microbiology 3.

Wahl, Ramon, Wippel, Kathrin, Goos, Sarah, Kämper, Jörg, and Sauer, Norbert. "A novel high-affinity sucrose transporter is required for virulence of the plant pathogen Ustilago maydis." PLoS biology 8.2 (2010): e1000303.

Walsh, MICHAEL C, Smits, HANS P, Scholte, MARCEL, and Van Dam, K.

"Affinity of glucose transport in Saccharomyces cerevisiae is modulated during growth on glucose." Journal of Bacteriology 176.4 (1994): 953-958.

Wang, Chengqiang, Bao, Xiaoming, Li, Yanwei, Jiao, Chunlei, Hou, Jin, Zhang, Qingzhu, Zhang, Weixin, Liu, Weifeng, and Shen, Yu. "Cloning and characterization of heterologous transporters in Saccharomyces cerevisiae and identification of important amino acids for xylose utilization."

Metabolic engineering 30: 79-88.

Wei, Liang, Liu, Jiao, Qi, Haishan, and Wen, Jianping.

"Engineering Scheffersomyces stipitis for fumaric acid production from xylose."

Bioresource technology 187: 246-254.

Weierstall, Thomas, Hollenberg, Cornelis P, and Boles, Eckhard.

"Cloning and characterization of three genes (SUT1-3) encoding glucose transporters of the yeast Pichia stipitis."

Molecular microbiology 31.3 (1999): 871-883.

Weusthuis, Ruud A, Visser, Wiebe, Pronk, Jack T, Scheffers, W Alexander, and van Dijken, Johannes P.

"Effects of oxygen limitation on sugar metabolism in yeasts: a continuous-culture study of the Kluyver effect." Microbiology 140.4 (1994): 703-715.

Wieczorke, Roman, Krampe, Stefanie, Weierstall, Thomas, Freidel, Kerstin, Hollenberg, Cornelis P, and Boles, Eckhard. "Concurrent knock-out of at least 20 transporter genes is required to block uptake of hexoses in Saccharomyces cerevisiae." FEBS letters 464.3 (1999): 123-128.

Wright, Barbara E, Longacre, Angelika, and Reimers, Jacqueline.

"Models of Metabolism in Rhizopus oryzae."

Journal of theoretical biology 182.3 (1996): 453-457.

Wu, MIAN and Tzagoloff, Alexander.

"Mitochondrial and cytoplasmic fumarases in Saccharomyces cerevisiae are encoded by a single nuclear gene FUM1."

Journal of Biological Chemistry 262.25 (1987): 12275-12282.

$\mathrm{Xu}$, Guoqiang, Chen, Xiulai, Liu, Liming, and Jiang, Linghuo.

"Fumaric acid production in Saccharomyces cerevisiae by simultaneous use of oxidative and reductive routes."

Bioresource technology 148: 91-96.

$\mathrm{Xu}$, Guoqiang, Liu, Liming, and Chen, Jian.

"Reconstruction of cytosolic fumaric acid biosynthetic pathways in Saccharomyces cerevisiae."

Microbial cell factories 11.1 (2012a): 24.

Xu, Guoqiang, Zou, Wei, Chen, Xiulai, Xu, Nan, Liu, Liming, and Chen, Jian.

"Fumaric acid production in Saccharomyces cerevisiae by in silico aided metabolic engineering." PLoS One 7.12 (2012b): e52086.

Yang, Lei, Lübeck, Mette, and Lübeck, Peter S.

"Aspergillus as a versatile cell factory for organic acid production."

Fungal Biology Reviews .

Yin, Xian, Li, Jianghua, Shin, Hyun-dong, Du, Guocheng, Liu, Long, and Chen, Jian.

"Metabolic engineering in the biotechnological production of organic acids in the tricarboxylic acid cycle of microorganisms: Advances and prospects."

Biotechnology advances 33.6 (2015): 830-841.

Yin, Xian, Shin, Hyun-dong, Li, Jianghua, Du, Guocheng, Liu, Long, and Chen, Jian.

"Comparative genomics and transcriptome analysis of Aspergillus niger and metabolic engineering for citrate production." Scientific reports 7: 41040 .

Young, Eric M, Comer, Austin D, Huang, Huashu, and Alper, Hal S.

"A molecular transporter engineering approach to improving xylose catabolism in Saccharomyces cerevisiae."

Metabolic engineering 14.4 (2012): 401-411.

Young, Eric M, Tong, Alice, Bui, Hang, Spofford, Caitlin, and Alper, Hal S.

"Rewiring yeast sugar transporter preference through modifying a conserved protein motif."

Proceedings of the National Academy of Sciences 111.1 (2014): 131-136.

Zeng, Hong, Parthasarathy, R, Rampal, Amrit L, and Jung, Chan Y.

"Proposed structure of putative glucose channel in GLUT1 facilitative glucose transporter."

Biophysical journal 70.1 (1996): 14-21.

Zhang, Baohua, Skory, Christopher D, and Yang, Shang-Tian.

"Metabolic engineering of Rhizopus oryzae: effects of overexpressing pyc and pepc genes on fumaric acid biosynthesis from glucose." 
Metabolic engineering 14.5 (2012): 512-520.

Zhang, Baohua and Yang, Shang-Tian.

"Metabolic engineering of Rhizopus oryzae: effects of overexpressing fumR gene on cell growth and fumaric acid biosynthesis from glucose."

Process biochemistry 47.12 (2012): 2159-2165.

Zhang, Huaiyuan, Wu, Chao, Wu, Qingyu, Dai, Junbiao, and Song, Yuanda.

"Metabolic flux analysis of lipid biosynthesis in the yeast Yarrowia lipolytica using 13C-labled glucose and gas chromatographymass spectrometry."

PloS one 11.7 (2016): e0159187.

Zhang, Lisha, Thiewes, Harry, and van Kan, Jan AL.

"The D-galacturonic acid catabolic pathway in Botrytis cinerea."

Fungal genetics and biology 48.10 (2011): 990-997.

Zhang, Ting, Wang, Zening, Deng, Li, Tan, Tianwei, Wang, Fang, and Yan, Yajun.

"Pull-in urea cycle for the production of fumaric acid in Escherichia coli."

Applied microbiology and biotechnology 99.12 (2015): 5033-5044.

Zhang, Weixin, Kou, Yanbo, Xu, Jintao, Cao, Yanli, Zhao, Guolei, Shao, Jing, Wang, Hai, Wang, Zhixing, Bao, Xiaoming, Chen, Guanjun, et al.

"Two major facilitator superfamily sugar transporters from Trichoderma reesei and their roles in induction of cellulase biosynthesis."

Journal of Biological Chemistry 288.46 (2013): 32861-32872.

Zhang, ZY, Jin, B, and Kelly, JM.

"Production of lactic acid and byproducts from waste potato starch by Rhizopus arrhizus: role of nitrogen sources."

World Journal of Microbiology and biotechnology 23.2 (2007): 229-236. 

SUMMARY

The aim of this thesis was to increase the understanding of organic acid production in Aspergillus niger and other filamentous fungi, with the ultimate purpose to improve $A$. niger as biotechnological production host.

In Chapter 1, the use of microbial cell-factories for the production of various compounds of interest, with a focus on organic acid production in A. niger, is introduced.

To convert $A$. niger into a cell-factory for the production of fumarate, an organic acid that this fungus does not naturally accumulate extracellularly, we need to know the key components that lead to high extracellular fumarate accumulation. This can be achieved by studying a natural fumarate producer, in our case the filamentous fungus Rhizopus delemar. To increase both the understanding of $R$. delemar fumarate production, and identify a possible candidate fumarate exporter protein for heterologuous expression in $A$. niger, we studied differences in the transcriptional and proteomic responses of $R$. delemar under high and low fumarate producing conditions, described in Chapter 2. Based on our analyses, we propose that a substantial part of the fumarate accumulated in $R$. delemar during nitrogen starvation results from the urea cycle due to amino acid catabolism. Thus, although we failed to identify the correct fumarte exporter (discussed in Chapter 8), the results of these analyses lead to a broader understanding of the mechanism underlying fumarate accumulation in $R$. delemar.

In order to make $A$. niger a suitable production host for other organic acids, we also delved deeper into the understanding of why A. niger has an innate ability to secrete various organic acids, especially citrate, described in Chapter 3. We show that an increase in citrate secretion under iron limited conditions is a physiological response consistent with a role of citrate as $A$. niger iron siderophore. We found that $A$. niger citrate secretion increases with decreasing amounts of iron added to the culture medium and, in contrast to previous findings, this response is independent of the nitrogen source. Differential transcriptomics analyses of the two A. niger mutants NW305 (gluconate non-producer) and NW186 (gluconate and oxalate non-producer) revealed up-regulation of the citrate biosynthesis 
gene citA under iron limited conditions compared to iron replete conditions. In addition, we show that $A$. niger can utilise Fe(III) citrate as iron source. Finally, we discuss our findings in the general context of the $\mathrm{pH}$-dependency of $A$. niger organic acid production, offering an explanation, besides competition, for why $A$. niger organic acid production is a sequential process influenced by the external $\mathrm{pH}$ of the culture medium.

In Chapter 4, we further unravel the various different mechanisms underlying extracellular A. niger citrate accumulation. We show that the phenotype of increased extracellular citrate accumulation can have fundamentally different underlying mechanisms, depending on how this response was triggered. We found that varying the amount and supplement of an arginine auxotrophic $A$. niger strain induces increased citrate productivity. Transcriptomics analysis shows down-regulation of citrate metabolising enzymes in the conditions in which more citrate is accumulated extracellularly. This contrasts with the transcriptional adaptations triggered by iron limited conditions, described in Chapter 3. By combining data obtained from both experimental setups described in Chapters 3 and 4, we compiled a list of likely citrate transporter candidates. Two promising citrate exporter candidates were tested in the yeast Saccharomyces cerevisiae, of which one was successfully identified as citrate exporter. Our findings provide the first steps in untangling the complex interplay of different mechanisms underlying $A$. niger citrate accumulation, and we pinpoint, for the first time, a promising $A$. niger citrate exporter candidate, offering a valuable tool for improvement of $A$. niger as biotechnological cell-factory for citrate production.

For the identification of different $A$. niger substrate importers, we combined in silico and in vivo approaches, and established a reliable pipeline to identify and test candidate transport proteins. The in silico approach, in which likely glucose transporter candidates are inferred from good matches with a glucose transporter specific Hidden Markov model $\left(\mathrm{HMM}_{\mathrm{gluT}}\right)$, and the in vivo approach, in which a sub-cellular proteomics approach is applied to isolate plasmalemmal glucose transporters, is described in Chapter 5. In the presented research work, a hidden Markov model (HMM), that shows a good performance in the identification and segmentation of functionally validated glucose transporters, was constructed. The model ( $\left.\mathrm{HMM}_{\text {gluT }}\right)$ was used to analyse the A. niger membrane-associated proteome response to high and low glucose concentrations at a low $\mathrm{pH}$. By combining the abundance patterns of the proteins found in the A. niger plasmalemma proteome with their $\mathrm{HMM}_{\text {gluT }}$ scores, two new putative high affinity glucose transporters, denoted MstG and MstH, were identified. MstG and MstH were functionally validated and biochemically characterised by heterologous expres- 
sion in a $S$. cerevisiae glucose transport null mutant. They were shown to be a high affinity glucose transporter $(\mathrm{Km}=0.6 \pm 0.1 \mathrm{mM})$ and a very high affinity glucose transporter $(\mathrm{Km}=0.06 \pm 0.005 \mathrm{mM})$ respectively.

The concepts developed in Chapter 5 were applied in Chapter 6 to identify further substrate importer proteins in both $A$. niger and another filamentous fungus, Trichoderma reesei. Again a hidden Markov model, this time for the identification of xylose transporters, was constructed and used to analyse the A. niger and T. reesei in silico proteomes, yielding a list of candidate xylose transporters. From this list, three A. niger (XltA, XltB and XltC) and three T. reesei (Str1, Str2 and Str3) transporters were selected, functionally validated and biochemically characterised through their expression in a $S$. cerevisiae hexose transport null mutant, engineered to be able to metabolise xylose, but unable to transport this sugar. All six transporters were able to support growth of the engineered yeast on xylose, but varied in affinities and efficiencies in the uptake of the pentose. Amino acid sequence analysis of the selected transporters showed the presence of specific residues and motifs associated to xylose transporters. Transcriptional analysis of $A$. niger and T. reesei showed that XltA and Str1 were specifically induced by xylose and dependent on the $\mathrm{X} \ln R / \mathrm{Xyr} 1$ regulators, implying a biological role for these transporters in xylose utilisation. Thus, our findings show that our approach using HMMs is a robust pipeline to identify different substrate importer candidates.

In Chapter 7, comparative plasmalemma proteomic analysis was used to identify candidate L-rhamnose transporters in A. niger. Further analysis was focused on protein ID 1119135 (RhtA) (JGI A. niger ATCC 1015 genome database). RhtA was classified as a Family 7 Fucose: $\mathrm{H}^{+}$Symporter (FHS) within the Major Facilitator Superfamily. Family 7 currently includes exclusively bacterial transporters able to use different sugars. Strong indications for its role in L-rhamnose transport were obtained by functional complementation of the Saccharomyces cerevisiae EBY.VW.4000 strain in growth studies with a range of potential substrates. Biochemical analysis using $\mathrm{L}-\left[{ }^{3} \mathrm{H}(\mathrm{G})\right]$-rhamnose confirmed that RhtA is a L-rhamnose transporter. The RhtA gene is located in tandem with a hypothetical alpha-L-rhamnosidase gene (rhaB). Transcriptional analysis of rhtA and rhaB confirmed that both genes have a coordinated expression, being strongly and specifically induced by L-rhamnose, and controlled by RhaR, a transcriptional regulator involved in the release and catabolism of the methyl-pentose. RhtA is the first eukaryotic L-rhamnose transporter identified and functionally validated to date.

In Chapter 8, the findings presented in this thesis with regards to our at- 
tempts at improving $A$. niger as biotechnological production host are summarised, and further implications for metabolic engineering approaches based on the conclusions drawn are discussed. 
Discipline specific

SB 6.0

BE-Basic annual meeting

Flagship meeting

Metabolomics for microbial systems biology

Basics of parameter estimation

$\mathrm{SB} @ \mathrm{NL}$

BE-Basic annual meeting

Flagship meeting

NBC-15 Biotechnology

BE-Basic annual meeting

ECO-BIO 2016

BioSB 2016

Metabolic Engineering 11

\section{General}

Data management

Systems biology: statistical analysis of -omics data

Project and time management

The essentials of Scientific writing and presenting

Career orientation

\section{Optional}

Research proposal

Group meetings

Seminars
2012

2013

2012

Year

2012

2013

2013

2013

2013

2013

2014

2014

2014

2015

2016

2016

2016

2013

2015

2012 - 2017

2012 - 2017 



\section{ACKNOWLEDGEMENTS}

Every $\mathrm{PhD}$ would be impossible to master without the support of other people, be it because they are directly involved with the scientific work, or for moral support in and outside of work.

First and foremost, I want to thank my supervisors Peter and Maria. Maria, thank you for being the reliable go-to person for pretty much everyone at SSB. I do not know how you can do it, but no matter the topic, or your official involvement in the project, you somehow manage to make time and give valuable feedback to whomever asks you for help. This gave rise to the increasingly common term in SSB: "Maria is helping me out, so it will be fine." I'm glad that asking you for help resulted in you eventually becoming one of my official supervisors. Working with you was not only enriching, but also a lot of fun, and made me want to achieve more; thank you for all your support in so many aspects of my $\mathrm{PhD}$. Peter, special thanks also to you for taking over as my official supervisor, and although this situation we suddenly found ourselves in was maybe difficult to get used to for both of us, I am really glad it turned out the way it did. Both being a bit headstrong, or plain out "control freaks", as you like to put it, we did have our fair share of intense scientific discussions. I am grateful to you that I could feel comfortable enough to know that we can discuss openly and honestly; thank you for guiding my $\mathrm{PhD}$ to completion, against the odds.

Undeniably another consistent helping force in my PhD, Juanan, I want to thank you for your unfaltering support and encouragement. Even after you left, I knew I could contact you any time if I needed help, and you always had my back not only in all my chapters and papers, but in all aspects related to my $\mathrm{PhD}$ in general. No matter the struggles and pitfalls - aka "The fumarate story" - we never gave up, and I think all our hard work related to the different projects we worked on together really paid off. Laura, I also want to thank you for your help and support, especially at the start of my $\mathrm{PhD}$. Back then, the wealth of information and expertise on Aspergillus niger that was present in our lab was overwhelming, and you helped me sort through it all. Thank you for sharing your knowledge and expertise, and smoothing out the path. Jasper, we started 
together, and getting the lab-perspective on the work was really enriching, as was working with you in general. Congrats on already having the Dr title at the time point of me writing this; je was me voor! Tom, not only my $\mathrm{PhD}$, but I think everyone working at the former Leo-group depended heavily on your expertise working with $A$. niger. Bedankt voor al je hulp, niet alleen voor het werk in relatie met het lab maar ook dat ik met jou gewoon Nederlands durf te praten om te oefenen. Also very special thanks to you, Merlijn. Your enthusiasm and willingness to stick through unpredictable, yet exciting, experiments is really great. Besides making the lab-parts of our projects work, you also corrected many of the typos (some multiple times) in our shared manuscripts, and it was always fun working with you. Nong, the last standing soldier of the fungal group, I am glad you are still keeping the $A$. niger culture alive a bit longer. And speaking of culture; your introduction of the home-made birthday-cards is a great way of making sure none of us can ignore their birthday and get away without bringing cake any more (especially those who share their birth date with you). Thank you also for all the fun dinners in which you re-defined the concept of "spicy" with your amazing cooking, and for teaching me a Thai word - mango - which apparently means "owl" in your language. Marta, we still got to work together after all, and your persistence to get the citrate transporter into Saccharomyces cerevisiae really made all the difference on that final project. Thank you for your spontaneous offer to help and especially for not giving up on it when things didn't work out as smoothly as expected. I also want to thank the BIFAP team, Francisca, Mihir and Kiira, amongst others. It was great sharing our work and experiences, although I sometimes wish this had resulted in more tangible collaborations. Finally, Niru, you might not be fully aware of this, but besides being a truly good friend and source of genuine laughter - not to be (purposefully) misunderstood - during all the coffee and lunch and spontaneous "I'm in your office now" breaks, you also contributed significantly to the work presented in Chapter 3 of this thesis. As you said, someone else drinking coffee benefited you, but someone else not drinking coffee benefited me just as much, I wonder if you can figure out why.

Without any doubt, the time spent to complete my thesis would not have been nearly as pleasant were it not for the good atmosphere we have at SSB. In this context, Vitor, thank you for taking the group dynamics into account when hiring people, for your efforts in fostering the wet-dry lab collaborations within SSB and between SSB and other labs, and for the freedom you give us at the department. And of course it is for those colleagues you hire that make all the difference. Rita, thank you for your tokens of support especially in the final months of my 
$\mathrm{PhD}$, and also for your advice on Dutch children's literature. Sharing an office with you was always nice, especially after it was somehow transformed into the chocolate office. Benoit, besides spending a lot of time in your office just sitting at the windowsill with a cup of coffee, or more recently trying to grow an apple tree from a seedling, I also enjoyed all our cheese-based dinners a lot. Finally someone that understands that you don't get a different type of cheese just by letting it age a bit longer, or by adding colourful ingredients. Bastian, without doubt the social core of the SSB group as I experienced it, your willingness to help whomever and whenever you can never ceases to amaze. Thank you also for always going out of your way to make sure really everyone feels included and is aware of the coffee breaks. Maarten, I would have to admit that said coffee breaks were a lot more entertaining when you were around, and I can also not deny that having someone compose your own, personal war-cry is quite special, if nothing else. Jesse, thank you for the often very insightful discussions about all kinds of aspects of life. Similarly, Niels, discussions with you also often led to new insights about various aspects of life and work, some more useful than others. In this regard, Emma, you certainly had a talent for bringing a new angle on many of the topics discussed during coffee and lunch, rivaled only by Bob in sheer curiousity. Rob, sharing some thoughts and hearing your opinion on matters was also always a treat, as was discovering your uncanny talent for acting. Nhung, thank you for so many things. For showing me the strengths of being positive, for your friendship and company, and for instilling in me the love - or should I call it addiction - to coconut water, although none can come close to the fresh one you brought from your parents' garden all the way from Vietnam. Linde oh Linde, in mijn minde, werd het lab creatiever toen je beginde. Bedankt voor je vertrouwen, waarop we vriendschap konden bouwen, ons dagje uit zal ik altijd in gedachten houden. Thanks Rik, for your dark humour and wit, thank you Nikolaos, for the comradeship, thank you Erika for your warmth and contagious laughter, thank you Anna, for the calm counterpoint to it all. Thank you Ruud, Edoardo, Tjerko, Rienk, Mark, Stamatios, Javi and Kal for making SSB what it is. Milad, not only the time at the department, but also our holidays together with Ruben were a chance to take our friendship to new depths (yes, I am re-using Ruben's pun on the diving here). Jasper, your hard working attitude is admirable in its own right, but even though that meant we didn't spend as much time over coffee, I want to thank you for your willingness to help whenever there was a weird computer issue. Speaking of which, Bart, thank you for solving the bigger issues, especially with the data management, and for making sure things were always ready when it was urgent. 
I also want to thank my students Gwen, Pepe, Nicolo, Mark, Bas and Joris for the enthusiasm and dedication for the various different student projects. In this regard: Michiel, Emiel, Marjan, Shreyans, Vincen, Marit, Jingjing and Danny, I have rarely seen such a bunch of hard working, intelligent students coming together, you made being part of the iGEM 2013 supervision team together with Mark and Ruben a real honour. Mark, Floor, thank you for enabling iGEM in Wageningen, where I first experienced how it is to be truly excited about science and research. Brendan, the time and effort we put into the oscillator project would have been quite different were it not for how well we got along and worked together. Besides valuing your friendship a lot, I dare say you and also Matthijn had a crucial role in shaping me as the scientist I am now. Working with you two and the rest of the Wageningen UR iGEM 2011 team was truly unique and enriching. Ruben, Youri, David, Martijn, Pim, Rob, Hugo, Suzette, Jaap, Erik, Shi and Mariana: thank you for this experience. Special thanks also to Carolien, Mirella, Anja, Philippe and Wim for making the SSB and MIB departments, which I have been sticking with since that first iGEM year, work so well together. Also to the colleagues from MIB, who became increasingly elusive once we moved to the new Helix building, Prarthana, Mark and Nico, thank you for the activities involving ice cream or feeling thoroughly inadequate next to you guys at pub quiz - yes, Mark, I have no idea how you distinguished the eggs of 10 different animals. Ioannis, Johanna, Indra, Irene, and Russian Alex, thanks for the good chats once in a while.

Teunke, it was always the time spent talking to you and sharing the enthusiasm for the songs that made going to Zumba so much fun and valuable to me. You understand and know things without much explanation. Thank you for your friendship, trust, encouragement and support throughout the years of the PhD. Alex, thank you not only for your company, especially at bouldering, where it made me feel slightly less singled out regarding matters of grace and elegance, but also for your advice and help regarding yeast growth in the lab. And of course, going to the ME 11 conference in Japan with you and Jasper was a lot of fun! Yuan my friend, our pursuit of the best coffee in (reasonably) close proximity to the lab was successful especially regarding our friendship that resulted from it. Talking to you was and is always straightforward, thank you for everything.

Michael, until the end I sometimes still wished you had prolonged the time spent on your $\mathrm{PhD}$ in Wageningen just to still be here until I am also (g/d)one. You predicted the way I would change my view on academia and my $\mathrm{PhD}$ quite correctly, and were a guiding friend on each step of the way. Thus, thank you for your support during and after my $\mathrm{PhD}$, especially also in the next step of 
moving to Heidelberg - together with Ruben, Wen, Margo and Sarah. Maybe that means that one day we will actually communicate in German, but who knows. I am glad we kept in touch, and speaking of keeping in touch; Wen, sunshine, you are truly amazing, fabulous, and bright-shining. From our first long chat because we both were borrowing Laura's WUR card to get coffee, to playing games, to bouldering, to travelling together, to you literally holding my hand when dropping of the draft of this thesis for official approval, you have been an amazing friend throughout. Thank you for everything, and much more in the years to come. Margo, the more I know you, the more I like you; thank you for so many days spent together bouldering or playing games, and for being such a good listener when needed most. Paul, thanks be also to you for the times spent playing games and travelling, and being the person to rely on no matter what. Being around you is always easy. Thomas, I don't know whether me or Ruben are happier about the two of you staying friends beyond high school, but its probably a close call. The game evenings, that became even more regular once you moved to Wageningen, canoeing in Finland together with Ruben and Sanne, but also just talking to you: het was alltijd gezellig. Bedankt voor alles en meer. Sanne, I think we took one look at each other and knew we would get along, and it has proven very true over the years. Oud- en nieuw and learning "Rikken" together from Thomas en Ruben, making many mistakes when we're tired, but always having a lot of fun while doing so: thank you.

Thanks for all the fun evenings and days playing games and laser tagging, Ruben, Margo, Wen, Paul, Michael, Thomas, Sanne, David, Maria, Nong, Bastian, Niru, Rajaram, Teunke, Mark, Tim - even though sometimes I doubt your choice of instant kills - and Femke. Bedankt Gerard, Mike, Ronald en Ivar voor de leuke Magictoernooien en spellendagen.

And to all the people who went bouldering, in the halls across the Netherlands or to Fontainebleau: Ruben, Wen, Michael, David, Floris, Margo, Alex and Thijs, thank you for those fun and challenging moments to counterbalance the work and effort put into the PhD.

Danke o da Lüt vom Ländle, wo mi vorallem moralisch unterstützt hend. Nicole, du bischt vo Afang a an Teil vo mim Abentüür do in Wageningen gse. Danke, dass du jedesmol dabei bischt, wenn i an neua Abschnett vo mim Leba afang. Elisabeth, danke för dini Bsüach und daför, dass du miar afacht so guat zualosa kascht. Danke o diar Amely, dass du mi gi Bsuacha ko bischt, för dini ufmunternda Wort und dass du in Basel a betz uf dr Ruben ufpasst hescht. Tatjana, danke för dis Vertraua i mini Fähigkeit, a Doktorat abschlüssa z’könna. Caroline, danke daför, dass miar üs doch nia ganz us da Oga verlürend. 
Nathalie, danke för's regelmässige Skypa und minder regelmässige Bruncha, wo miar üs noch Herzensluscht ustobt hend. Danke eu o afacht allgemein för euri Fründschaft.

Roger, Michaela, Manuela und Marion, so guat, dass miar d'Wiahnachtstreffa immer no jedes Johr durchzühen. Martina, miar hend o weder zuanand g'funda, und i bi froh drum. Dini Hilf und Unterstützig i da letschta paar Monat vo mim PhD hend miar wörkleg an Kraftschub ge, drum danke diar, am Andreas und natürlich eurem Gabriel.

Dorus, sharing a flat with you, with or without other room mates, was an interesting experience for various reasons. Not only your solutions to arising household issues, but also to life in general, was always refreshingly different and yet familiar. Spending time with you is meaningful no matter how much we have to say to each other at any given moment, and I want to thank you for all the good times and discussions we've had throughout the years.

Ruben, last but not least of my friends to thank for being by my side throughout this journey. We met during iGEM in 2011, and immediately got along. I remember one of the first things you asked me was whether I was also in doubt about how much I could contribute to the first Wageningen UR iGEM team, comprising so many amazing and motivated students. I guess we could, and look at us now. Living together, travelling together, playing board and card games together with other friends, but also just having coffee together; you were a consistent and reliable friend throughout, and you also made the Netherlands become "home" for me. Thank you for that, and everything else. Avalon, from when you were still a child until now seeing you as a young woman, it was always nice having you around and spending time with you. Thank you for your doubtless belief in me. Diny en Bert, bedankt dat ik me in jullie huis altijd thuis kon voelen.

Fabian, besides your general heart-felt support in everything I do, I also want to thank you for that (very urgent) packet of Swiss chocolate you sent me when I ran out of it in the last weeks of writing my thesis. I can say that that was a crucial factor that contributed significantly to the completion of my $\mathrm{PhD}$; thank you for that, and your unwavering belief in me. Ouma, thank you for always thinking of me and the beskuit you made especially for Yvonne to bring to us. Danke Hans, dass du an mich geglaubt hast. Yvonne and Walter, I don't think I can find the right words to tell you how much your support means to me. Not only that I know you will always have my back in everything I do, but you went beyond that. Thank you for everything.

Bedankt, Doretia, voor je hulp dat ik Leo zijn naam nog op mijn publicatie mocht zetten. Je zei dat hij zeker trots zou zijn geweest op deze publicatie en dat 
zijn betrokkenheid bij onze wetenschappelijke onderzoeken altijd groot was. Mijn thesis zou niet mogelijk zijn geweest zonder Leo. 
The research described in this thesis was financially supported by the IPOP program Systems Biology of Wageningen University \& Research. 
0

○

○

○

○

Q

○

○

-

-

9

○

○

○

○

-

○

○

O

○

○

○

C

○

○

○

○

○

○

-

○

0

-

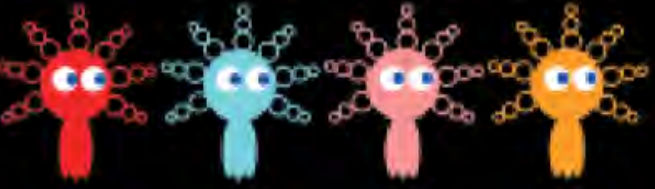

